\title{
New CTEQ global analysis of quantum chromodynamics with high-precision data from the LHC
}

\author{
Tie-Jiun Hou, ${ }^{1,}$ Jun Gao, ${ }^{2}$ T. J. Hobbs, ${ }^{3,4}$ Keping Xie, ${ }^{3,5}$ Sayipjamal Dulat, ${ }^{6, \$}$ Marco Guzzi, ${ }^{7}$ Joey Huston, ${ }^{8}$ \\ Pavel Nadolsky ${ }^{3,8}$ Jon Pumplin, ${ }^{8,}$ Carl Schmidt ${ }^{8},{ }^{8}$ Ibrahim Sitiwaldi, ${ }^{6}$ Daniel Stump, ${ }^{8}$ and C.-P. Yuan ${ }^{8, \|}$ \\ ${ }^{1}$ Department of Physics, College of Sciences, Northeastern University, Shenyang 110819, China \\ ${ }^{2}$ INPAC, Shanghai Key Laboratory for Particle Physics and Cosmology \& School of Physics \\ and Astronomy, Shanghai Jiao Tong University, Shanghai 200240, China \\ Center for High Energy Physics, Peking University, Beijing 100871, China \\ ${ }^{3}$ Department of Physics, Southern Methodist University, Dallas, Texas 75275-0181, USA \\ ${ }^{4}$ Jefferson Lab, EIC Center, Newport News, Virginia 23606, USA \\ ${ }^{5}$ PITT PACC, Department of Physics and Astronomy, University of Pittsburgh, \\ Pittsburgh, Pennsylvania 15260, USA \\ ${ }^{6}$ School of Physics Science and Technology Xinjiang University, Urumqi, Xinjiang 830046 China \\ ${ }^{7}$ Department of Physics, Kennesaw State University, \\ 370 Paulding Avenue, 30144 Kennesaw, Georgia 30061, USA \\ ${ }^{8}$ Department of Physics and Astronomy, Michigan State University, East Lansing, Michigan 48824, USA
}

(Received 20 February 2020; accepted 9 November 2020; published 11 January 2021)

\begin{abstract}
We present the new parton distribution functions (PDFs) from the CTEQ-TEA collaboration, obtained using a wide variety of high-precision Large Hadron Collider (LHC) data, in addition to the combined HERA I + II deep-inelastic scattering dataset, along with the datasets present in the CT14 global QCD analysis. New LHC measurements in single-inclusive jet production with the full rapidity coverage, as well as production of Drell-Yan pairs, top-quark pairs, and high- $p_{T} Z$ bosons, are included to achieve the greatest sensitivity to the PDFs. The parton distributions are determined at next-to-leading order and nextto-next-to-leading order, with each of these PDFs accompanied by error sets determined using the Hessian method. Fast PDF survey techniques, based on the Hessian representation and the Lagrange multiplier method, are used to quantify the preference of each data set to quantities such as $\alpha_{s}\left(m_{Z}\right)$, and the gluon and strange quark distributions. We designate the main resulting PDF set as CT18. The ATLAS 7 TeV precision $W / Z$ data are not included in CT18, due to their tension with other datasets in the global fit. Alternate PDF sets are generated including the ATLAS precision $7 \mathrm{TeV} W / Z$ data (CT18A), a new scale choice for low- $x$ DIS data (CT18X), or all of the above with a slightly higher choice for the charm mass (CT18Z). Theoretical calculations of standard candle cross sections at the LHC (such as the $g g$ fusion Higgs boson cross section) are presented.
\end{abstract}

DOI: 10.1103/PhysRevD.103.014013

\section{INTRODUCTION}

With an accumulated data sample of over $140 \mathrm{fb}^{-1}$ at the $13 \mathrm{TeV}$ run for both ATLAS and CMS collaborations, the Large Hadron Collider (LHC) has entered an era of

\footnotetext{
*Deceased

tjhou@msu.edu

\$sdulat@hotmail.com

§nadolsky@smu.edu

yuan@pa.msu.edu
}

Published by the American Physical Society under the terms of the Creative Commons Attribution 4.0 International license. Further distribution of this work must maintain attribution to the author(s) and the published article's title, journal citation, and DOI. Funded by SCOAP ${ }^{3}$. precision physics. The experimental precision has been matched by improvements to the theoretical predictions, with a number of collider processes now available at the next-to-next-to-leading order (NNLO) in the QCD coupling strength. Such precision is necessary for rigorous tests of the Standard Model (SM) and in searches for signs of physics beyond the Standard Model (BSM), as there have been no "smoking-gun" signs of BSM physics to date. Precise predictions in QCD theory require correspondingly precise parton distribution functions (PDFs) [1-8], which in turn warrant advances in interpreting LHC experiments to extract important information about the SM and, possibly, BSM physics.

To this end, we present a new family of CTEQ-TEA parton distribution functions, designated as CT18. These PDFs are produced at both next-to-leading order (NLO) 
and next-to-next-to-leading order (NNLO) in the QCD coupling constant, $\alpha_{s}$. The CT18 PDFs update those of the CT14 family presented in Ref. [1]. In the new analysis, we include a variety of new LHC data, at the center-of-mass energies of 7 and $8 \mathrm{TeV}$, on the production of singleinclusive jets, $W / Z$ bosons, and top-antitop quark pairs, obtained by the ATLAS, CMS, and LHCb collaborations. At the same time, the update retains crucial "legacy" data from the previous CT global QCD analyses, such as the HERA I + II combined data on deep-inelastic scattering (DIS) and measurements in fixed-target experiments and at the Fermilab Tevatron $p \bar{p}$ collider. Measurements of processes in similar kinematic regions, by both ATLAS and CMS, allow crucial cross-checks of the data. Measurements by LHCb often allow extrapolations into new kinematic regions not covered by the other experiments. Some processes, such as $t \bar{t}$ production, allow for the measurement of multiple observables that provide similar information for the determination of PDFs. In addition to the PDFs themselves, we also present relevant PDF luminosities and predictions with uncertainties for standard candle cross sections at the LHC.

The goal of the CT18 analysis is to include as wide a kinematic range for each measurement as possible while still achieving reasonable agreement between data and theory. For the ATLAS $7 \mathrm{TeV}$ jet data [9], for example, all rapidity intervals cannot be simultaneously used without the introduction of systematic error decorrelations provided by the ATLAS collaboration [10]. Even with that decorrelation, the resultant $\chi^{2}$ for the new jet experiments is not optimal, resulting in less effective PDF constraints. Inclusive cross section measurements for jet production have been carried out for two different jet-radius values, $R$, by both ATLAS and CMS. For both experiments, we have chosen the data with the larger $R$ value, for which the NNLO (fixed order) prediction should have a higher accuracy. We evaluate the jet cross section predictions using a QCD scale of inclusive jet transverse momentum $Q=p_{T}^{\text {jet }}$, consistent with past usage at NLO. The result is largely consistent with similar evaluations using $Q=H_{T}$ [11-13].

Theoretical predictions for comparison to the data used in the global fit have been carried out at NNLO, either indirectly through the use of fast interpolation tables such as fastNLO [14,15] and APPLGRID [16], together with NNLO/ NLO $K$ factors, or directly (for top-quark-related observables) through the use of fastNNLO grids $[17,18]$.

In an ideal world, all such datasets would perfectly be compatible with each other, but differences are observed that do result in some tension between datasets and pulls in opposite directions. One of the crucial aspects of carrying out a global PDF analysis is dealing with datasets that add some tension to the fits, while preserving the ability of the combined dataset to improve on the existing constraints on the PDFs. In some cases, a dataset may be in such tension as to require either its removal from the global analysis or its inclusion only in a separate iteration of the new PDF set.

In this paper, we will describe how the high-precision ATLAS $7 \mathrm{TeV} W / Z$ rapidity distributions, which, as we find, favor an increase of the strange quark distribution at low $x$, require such special treatment. In particular, while other PDF-analysis groups (e.g., MMHT, see Ref. [19]) have noted that these ATLAS $W / Z$ data can be fitted with $\chi^{2} /$ d.o.f. that is comparable to the CT18 one, we find that such $\chi^{2}$ reflects systematic tensions with many of the other data in our global analysis. Furthermore, the standard Hessian profiling technique used by the experimental collaborations significantly underestimates the minimal $\chi^{2}$ that can be reached for the ATLAS $7 \mathrm{TeV} W / Z$ data when they are included in the CT18 fit. We therefore treat these measurements separately in an alternative fit, CT18A, introduced in Sec. II C. In another variant, CT18X, a special scale $\mu_{F, x}^{2} \equiv 0.8^{2}\left(Q^{2}+0.3 \mathrm{GeV}^{2} / x_{B}^{0.3}\right)$ is used for the calculation of low- $x$ DIS cross sections; the scale mimics the impact of low- $x$ resummation. Both modifications cause an increase in the low- $x$ quark and gluon distributions. Finally, these two variants of the CT18 fit are amalgamated into a combined alternative fit, CT18Z. Since the CT18Z PDFs are most dissimilar from the CT18 ones, we show numerous results based on the CT18 and CT18Z PDFs throughout the article, while deferring the comparisons to CT18A and X to Appendix A, where additional in-depth comparisons to CT18Z are also provided. A recommendation on selecting one of the four PDF ensembles depending on the user's needs is given at the beginning of Sec. VII.

Our current global analyses are carried out in four stages. First, PDFSENSE [20,21], a program for a rapid survey of QCD data using the Hessian approach [22,23], is used to select the datasets that are expected to have the greatest impact on the global PDF sets. This selection takes into account the sensitivity of the data to specific PDFs in a given $x$ range, which reflects both the correlation of these data with a given PDF, as well the size of the dataset and magnitudes of its statistical and correlated systematic errors. For example, both the collider inclusive jet data and the top-quark data have a strong correlation with the high- $x$ gluon, but the inclusive jet data has a larger sensitivity due to a much larger number of data points. Next, EPUMP [24,25] is used to quickly examine the quantitative impact of each selected dataset, within the Hessian approximation. Third, the full global PDF fit is carried out using all such datasets. Recent enhancements to the CT global analysis code have greatly improved the speed of the calculations. Last, the impact of key datasets on certain PDFs at specific kinematic points of interest, as well as on the value of $\alpha_{s}\left(M_{Z}\right)$, is assessed using the Lagrange multiplier (LM) method [26]. In order to minimize any parametrization bias, we have tested different parametrizations for CT18: e.g., using a more flexible parametrization for the strange quark PDF. In some 
kinematic regions, there are fewer constraints from the data on certain PDFs. In particular, LM constraints have been applied to limit the strangeness PDF at $x<10^{-5}$ to physically reasonable values, as summarized in Appendix C.

Our paper is organized as follows. Section II begins with an executive summary of the key stages and results of the CT18 global analysis. It continues with an overview of the chosen experimental data and alternative fits (CT18Z, CT18A, and CT18X) in the CT18 release. This section concisely summarizes the key results that are of interest to most readers. The subsequent sections and Appendixes elaborate on specific aspects and outcomes.

In Sec. III we detail theoretical/computational updates to the CT fitting methodology and details for specific processdependent calculations. Section IV presents the main results obtained in CT18 - the fitted PDFs as functions of $x$ and $Q$, the determinations of QCD parameters $\left(\alpha_{s}, m_{c}\right)$, the calculated parton luminosities, and the various PDF moments and sum rules. These comparisons will be of interest to a broad group of researchers who will use the PDFs for theoretical predictions at LHC experiments.

Section V describes the ability of CT18 to provide a successful theoretical description of the fitted data. In addition to characterizing the fit of individual datasets, in Sec. VI, we also compute the various standard candle quantities of relevance to LHC phenomenology, for instance, Higgs boson production cross sections at 13 and $14 \mathrm{TeV}$, and various correlations among electroweak boson and top-quark pair production cross sections. In Sec. VII, we discuss the broader implications of this work and highlight our main conclusions.

Several Appendixes present a number of important supporting details. In Appendix A, we review the CT18Z and other alternative fits, including descriptions of various datasets admitted into these separate analyses. A number of more formal details related to our likelihood functions and relations among covariance matrices are summarized in Appendix B. Appendix C presents the analytical fitting form adopted in CT18 and best-fit values of the PDF parameters. Appendix D presents a number of technical advances in the CT fitting framework, including code parallelization, while Appendix E enumerates the decorrelation models utilized in fitting the newly included inclusive jet data from the LHC. In Appendix F, we present the results of a short study based on Hessian profiling methods to assess the impact of the $7 \mathrm{TeV} W / Z$ production data taken by ATLAS. Additional figures are included as Supplemental material [27].

\section{OVERVIEW OF THE CT18 GLOBAL QCD ANALYSIS}

\section{A. Executive summary}

\section{Input experimental data and final PDF ensembles}

The CT18 analysis updates the widely used CT14 PDF sets [1] by applying NNLO and NLO global fits to an expanded set of experimental measurements that include high-statistics data from the $e p$ collider HERA and the LHC. The CT18 experimental dataset includes highstatistics measurements from ATLAS, CMS, and LHCb on production of inclusive jets, $W / Z$ bosons, and top-quark pairs, while it retains the crucial legacy data, such as the HERA run I and run II combined data and measurements from the Tevatron. By 2018, the LHC collaborations published about three dozen experimental datasets that can potentially constrain the CT PDFs. We selected the most promising experiments available by mid-2018 using the methods reviewed in Secs. II A 3 and II A 4. We then extensively examined the impacts of the datasets within the full fitting framework. Section II B contains an overview of these experiments. The kinematic distribution of the data points included in CT18 is shown in Fig. 1 as a function of the typical parton momentum fraction, $x$, and QCD factorization scale, denoted here as $Q .{ }^{1}$ As has been true for global PDF fits for some time, the data included cover a large kinematic range, both in $x$ and $Q$.

In light of the unprecedented precision reached in some measurements, the latest LHC data must be analyzed using NNLO theoretical predictions in perturbative QCD. The fitted PDFs we obtain in this analysis are plotted in Fig. 2, which displays in the upper panels the CT18 PDFs at two widely separated scales, $Q=2$ and $100 \mathrm{GeV}$ (on the left and right, respectively). In the lower panels, we show the corresponding PDFs found in our amalgamated alternative analysis, CT18Z.

The final CT18(Z) data ensemble contains a total of $N_{p t}=3681$ (3493) data points and results in $\chi^{2} / N_{p t}=$ $1.17(1.19)$ at NNLO. The PDF uncertainties are constructed at the $90 \%$ probability level based on two tiers of criteria as in the CT14 global analysis [1]. These PDFs are obtained by assuming a world-average QCD coupling constant, $\alpha_{s}\left(M_{Z}\right)=0.118$ [28]. The combined PDF $+\alpha_{s}$ uncertainty can be computed using the special $\alpha_{s}$ series of the PDFs for each family by adding the PDF and $\alpha_{s}$ uncertainties in quadrature, as explained in Ref. [29].

Among the four ensembles (CT18, A, X, and Z) of PDFs, the CT18 and CT18Z ensembles are the most dissimilar in terms of the shapes of PDFs, notably in the $x$ dependence of the fitted gluon and strangeness distributions, $g(x, Q)$ and $s(x, Q)$, as well as in some PDF uncertainties. For CT18, we obtain modest improvements in the precision for the gluon density $g(x, Q)$, as compared to CT14, following the inclusion of the LHC run-1 data discussed below. For CT18Z, however, we obtain a somewhat enlarged uncertainty for the gluon and perturbatively generated charm PDFs, especially at the lowest values of $x<10^{-3}$, due to the modified treatment of the DIS data described in Sec. II C and Appendix A. These final PDFs

\footnotetext{
${ }^{1}$ The typical momentum fractions and factorization scales are estimated as in Ref. [20].
} 


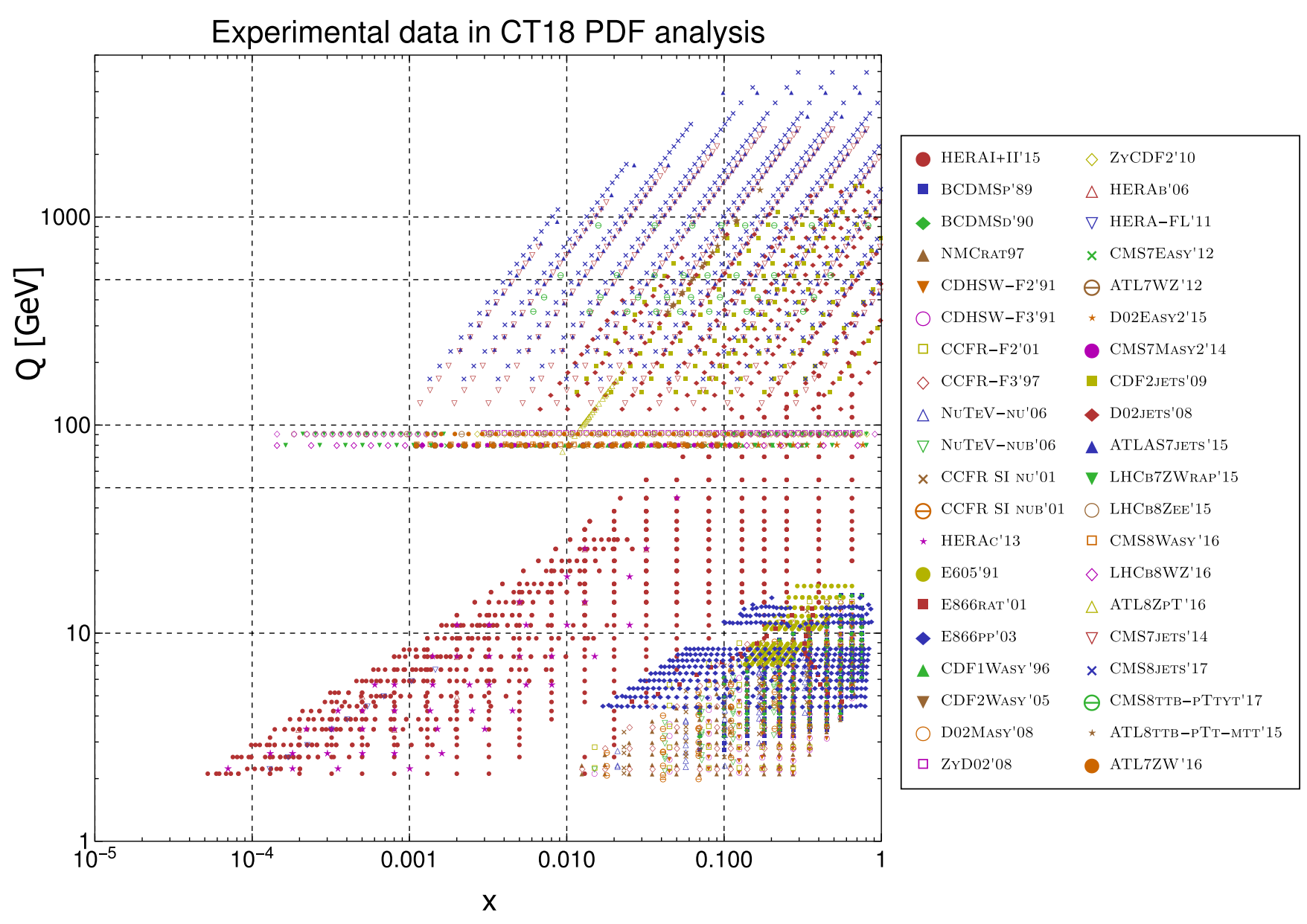

FIG. 1. The CT18 dataset, represented in a space of partonic $(x, Q)$, based on Born-level kinematical matchings, $(x, Q)=\left(x_{B}, Q\right)$, in DIS, etc. The matching conventions used here are described in Ref. [20]. Also shown are the ATLAS 7 TeV $W / Z$ production data (Exp. ID = 248), labeled ATL7WZ'12, fitted in CT18Z.

depend on numerous systematic factors in the experimental data. Scrupulous examination of the systematic effects was essential for trustworthy estimates of PDF uncertainties, and the scope of numerical computations also needed to be expanded.

\section{Combined HERA I + I I DIS data and the $x_{B}$-dependent factorization scale}

Even in the LHC era, DIS data from the $e p$ collider HERA provide the dominant constraints on the CT18 PDFs. This dominance is revealed by independently applying the EPUMP, PDFSENSE, and Lagrange multiplier methods. CT18 implements the final ("combined") dataset from DIS at HERA run-I and run-II [30], which supersedes the HERA run-I only dataset [31] used in CT14 [1]. A transitional PDF set, CT14 HERAII, was released based on fitting the final HERA data [32]. We found fair overall agreement of the HERA I + II data with both CT14 and CT14 $4_{\text {HERAII }}$ PDFs, and that both PDF ensembles describe equally well the non-HERA data included in our global analysis. At the same time, we observed some disagreement ("statistical tension") between the $e^{+} p$ and $e^{-} p$ DIS cross sections of the HERA I + II dataset. We determined that, at the moment, no plausible explanation could be provided to describe the full pattern of these tensions, as they are distributed across the whole accessible range of Bjorken $x$ and lepton-proton momentum transfer $Q$ at HERA. Extending these studies using the CT18 fit, we have investigated the impact of the choice of QCD scales on inclusive DIS data in the small- $x_{B}$ region, as will be explained later in Sec. II C.

We find that the quality of fit to HERA data is improved by about 50 units by evaluating the NNLO theoretical cross sections in DIS with a special factorization scale, $\mu_{F, x}$, that depends on Bjorken $x_{B}$ (not the momentum fraction $x$ ) and is introduced in Sec. II C. Figure 3 (left) shows the changes in the candidate CT18 PDFs obtained by fitting the DIS datasets with the factorization scale $\mu_{F, x}$, as compared to the CT18 PDFs with the nominal scale $\mu_{F}=Q$. With the scale $\mu_{F, x}$, we observe reduced $u$ and $d$ (anti)quark PDFs and increased gluon and strangeness PDFs at $x<10^{-2}$, as compared to the nominal CT18 fit, with some compensating changes occurring in the same PDFs in the 

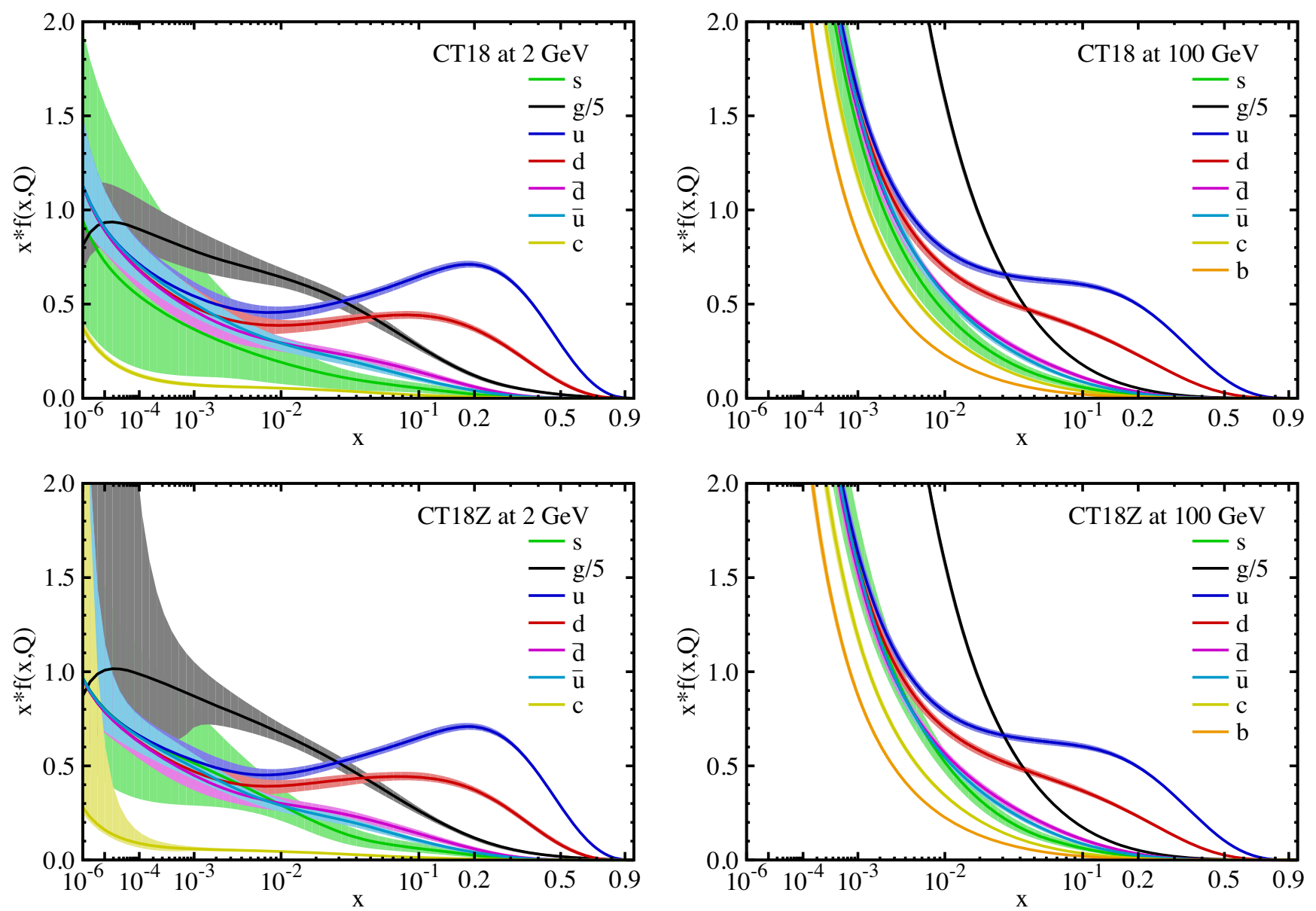

FIG. 2. Upper panels: The CT18 parton distribution functions at $Q=2 \mathrm{GeV}$ and $Q=100 \mathrm{GeV}$ for $u, \bar{u}, d, \bar{d}$, $s=\bar{s}$, and $g$. Lower panels: the analogous curves, but obtained for CT18Z. In all instances, the gluon PDF has been scaled down as $g(x, Q) / 5$. The charm distribution, $c(x, Q)$, which is perturbatively generated by evolving from $Q_{0}=1.3$ and $1.4 \mathrm{GeV}$, respectively, in CT18 and CT18Z, is also shown.
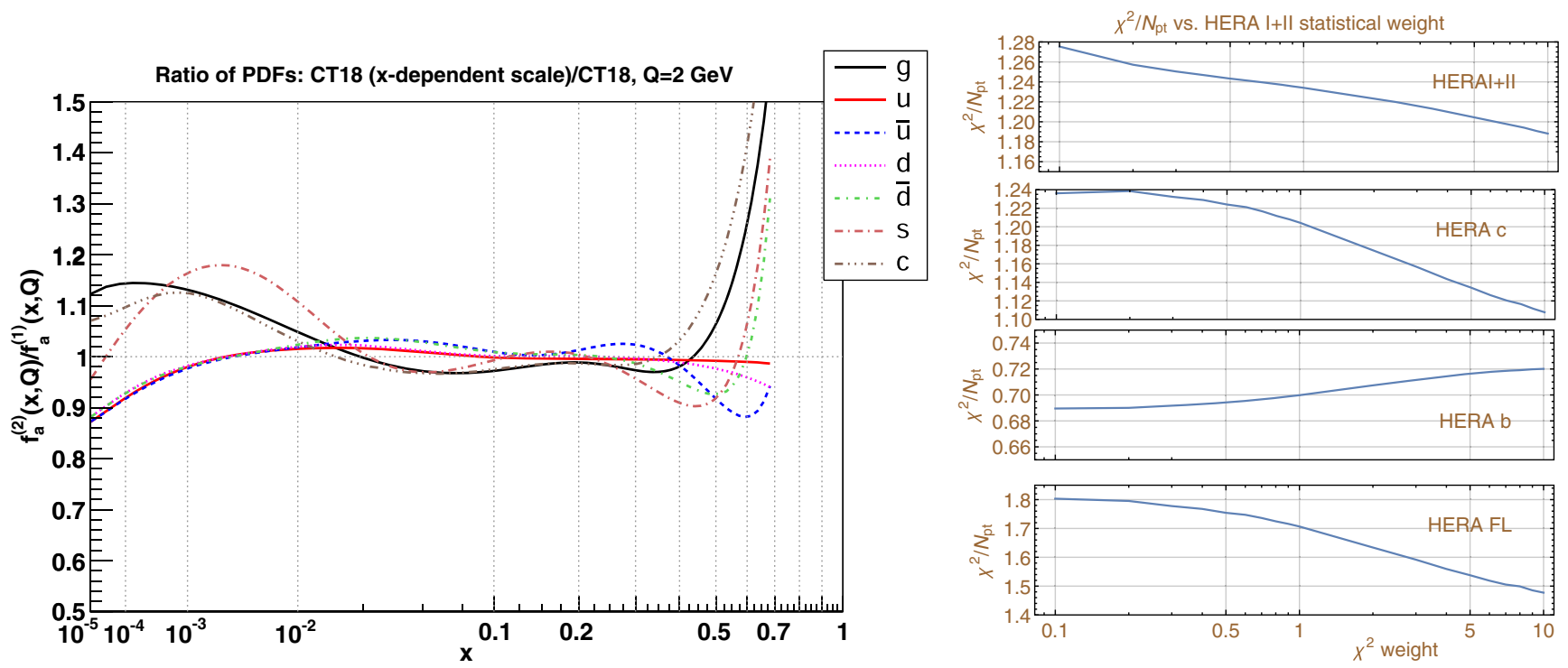

FIG. 3. Left: the ratios of the candidate CT18 NNLO PDFs obtained with the $x_{B}$-dependent and standard factorization scales in DIS datasets. Right: the $\chi^{2} / N_{p t}$ values for four HERA datasets in the CT18Z fit with the $x_{B}$-dependent DIS factorization scale and varied statistical weight of the HERA I + II inclusive DIS dataset. 
unconstrained region $x>0.5$ in order to satisfy the valence and momentum sum rules.

The right panel of Fig. 3 shows the $\chi^{2} / N_{p t}$ values $\left(\chi^{2}\right.$ divided by the number, $N_{p t}$, of experimental data points) for four HERA datasets (inclusive neutral and charged current DIS [30], reduced charm, bottom production cross sections, and $\mathrm{H} 1$ longitudinal function $F_{L}\left(x_{B}, Q^{2}\right)$ [33]) in the fits as a function of the statistical weight $w$ of the HERA I + II inclusive DIS dataset [30]. The default CT18Z fit corresponds to $w=1$; with $w=10$, the CT18Z fit increasingly behaves as a HERA-only fit. We see that, with the scale $\mu_{F, x}^{2}$ and $w=10, \chi^{2} / N_{p t}$ for the inclusive DIS dataset improves almost to the levels observed in the "resummed" HERA-only fits without intrinsic charm [34,35]. The quality of the fit to the charm semi-inclusive DIS (SIDIS) cross section and $\mathrm{H} 1 F_{L}$ also improves. ${ }^{2}$

The new combined charm and bottom production measurements from the $\mathrm{H} 1$ and ZEUS collaborations published in Ref. [36] (2018) have been investigated. In their current version, when these measurements replace the previous ones in the CT18 global analysis, they cannot be fitted with a reasonable $\chi^{2}$. Moreover, a mild tension is observed between these new combined data and several CT18 datasets such as the LHCb 7 and $8 \mathrm{TeV} W / Z$ production data, $Z$-rapidity data at CDF run-II, CMS $8 \mathrm{TeV}$ single inclusive jet production, and $t \bar{t}$ double differential $p_{T}$ and $y$ cross section. Therefore, we decided not to include these data in the CT18 global analysis as they require a dedicated investigation. In the H1+ZEUS analysis of Ref. [36], the $\chi^{2}$ for these measurements is also found not to be optimal. This is ascribed to a difference in the slope between data and theory in the intermediate/small $x$ region. In our attempt to fit the new combined charm and bottom production measurements, we have noticed a preference for a harder gluon at intermediate/small $x$. We are currently investigating these data separately and, in particular, we are exploring the impact of the new correlated systematic uncertainties, as their number increased from 42 in the old version of the data, to 167 in the new version. The results of this new study are going to be published in a separate forthcoming paper.

\section{Selection of new LHC experiments}

When selecting the most promising LHC experiments for the CT18 fit, we had to address a recurrent challengethe presence of statistical tensions among various (sub)sets of the latest experimental data from HERA, LHC, and the

\footnotetext{
${ }^{2}$ The use of the separate $\mathrm{H} 1 F_{L}$ data as well as the HERA-II combined data introduces some double counting. However, we have checked that this choice does not appreciably change the PDFs, while it does provide a useful indicator of the goodness of fit in the small- $x$ region. In particular, it is telling that the total $\chi^{2}$ value of $\mathrm{H} 1 F_{L}$ data (Exp. ID $=169$ ) becomes smaller, not larger, in the CT18X and CT18Z fits, as compared to CT18.
}

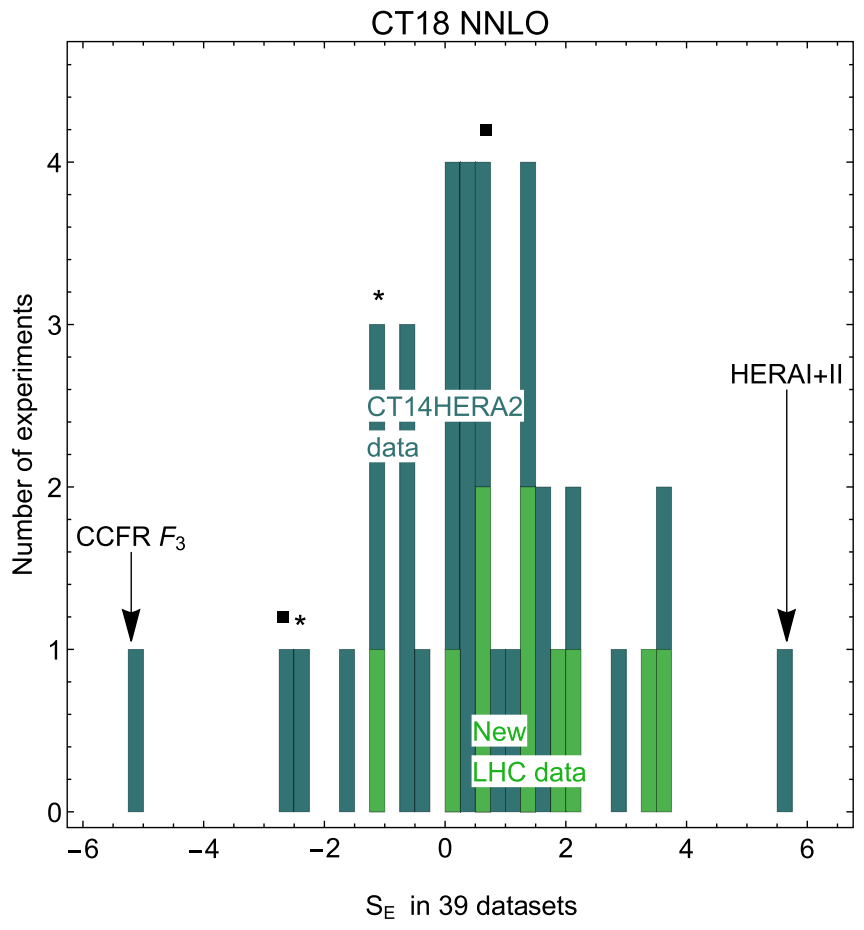

FIG. 4. A histogram of the effective Gaussian variable $\left(S_{E}\right)$ distributed over all CT18 datasets. Two squares and two stars indicate the $S_{E}$ values for the NuTeV dimuon and CCFR dimuon data, respectively.

Tevatron. The quickly improving precision of the collider data reveals previously irrelevant anomalies either in the experiment or theory. These anomalies are revealed by applying strong goodness-of-fit tests [37]. Figure 4 illustrates the degree of tensions using a representation based on the effective Gaussian variables $S_{E} \equiv \sqrt{2 \chi_{E}^{2}}-\sqrt{2 N_{E}-1}$ [38] constructed from the $\chi^{2}$ values and numbers of data points $N_{E}$ for individual datasets $E$. In an ideal fit in which the differences between theory and data are consistent with Gaussian random fluctuations, the probability distribution for $S_{E}$ must be approximately a standard normal distribution (with a unit half-width). In the global fits by CTEQTEA and external groups, we rather observe wider $S_{E}$ distributions as in Fig. 4, with some of the more comprehensive and precise datasets (notably, HERA I + II inclusive DIS [30] and ATLAS $7 \mathrm{TeV} W / Z$ production [39]) having $S_{E}$ values as high as five units or more. The question, then, is how to select clean and accurate experiments for the global analysis from an ever-growing list of measurements, while maximally preserving the consistency of the selected experiments. For example, there are many LHC datasets [40] that are potentially sensitive to the PDFs, including novel measurements involving the production of high- $p_{T} Z$ bosons, $t \bar{t}$ pairs, isolated photon, and small- $x$ heavy flavor (charm or bottom) quarks. Including all such candidate experiments into the full global fit is impractical: $\mathrm{CPU}$ costs grow quickly with the number of experimental datasets at NNLO. Poorly fitted experiments would 
increase, not decrease, the final PDF uncertainty. The generation of one error PDF set took several days of CPU time in the CT14 fit to 33 experiments in single-thread mode. Adding 20-30 additional experiments with this setup was thus impossible. The CTEQ-TEA group resolved these challenges through a multipronged effort that allowed us to include 11 new LHC datasets at 7 and $8 \mathrm{TeV}$ on $W^{ \pm}$, $Z$, jet, and $t \bar{t}$ production.

\section{Advances in fitting methodology}

To identify the eligible experimental datasets for the global fit, we developed two programs for fast preliminary analysis. The PDFSENSE program [20] was developed at Southern Methodist University (SMU) to predict quantitatively, and before doing the fit, which datasets will have an impact on the global PDF fit. The EPUMP program [24] developed at Michigan State University (MSU) applies Hessian profiling to quickly estimate the impact of data on the PDFs prior to the global fit. These programs provide helpful guidelines for the selection of the most valuable experiments based entirely on the previously published Hessian error PDFs. Section II B 1 demonstrates an application of PDFSENSE.

As we will discuss in Appendix F, the out-of-the-box algorithm for Hessian profiling implemented in the commonly used version 2.0.0 of the xFitter program [41] is inconsistent with the CTEQ-TEA definitions of PDF uncertainties and has predicted too optimistic $\chi^{2}$ values and PDF uncertainties in a number of studies for profiling the CTEQ-TEA PDFs. The EPUMP program does not have this caveat. Its Hessian updating algorithm better reproduces the $\chi^{2}$ values of the datasets in the full CT14 and CT18 fits, as well as the respective PDF uncertainties defined according to the two-tier definition of $\chi^{2}$ adopted in the CTEQ-TEA analyses since CT10 NLO [42].

The CTEQ fitting code was parallelized to allow a faster turnaround time (one fit within a few hours instead of many days) on high-performance computing clusters. For as much relevant LHC data as possible, we computed the NLO cross sections with the APPLGRID/fastNLO tables [16,43] (to be multiplied by tabulated point-by-point NNLO/NLO $K$-factor corrections) for various new LHC processes: production of $W / Z$ bosons, high- $p_{T} Z$-bosons, and inclusive jets; the NNLO cross section with the fastNNLO tables $[17,18]$ for the $t \bar{t}$ pair production at the LHC. The APPLGRID tables were cross validated against similar tables from other groups (available in the public domain) and optimized for speed and accuracy.

\section{Estimates of theoretical and parametrization uncertainties}

Significant effort was spent on understanding the sources of PDF uncertainties. Theoretical uncertainties associated with the scale choice were investigated for the affected processes, such as DIS as well as inclusive jet and high- $p_{T}$ $Z$ boson production. Other considered theoretical uncertainties were due to the differences among the NNLO and resummation codes (e.g., DYNNLO [44,45], MCFM [46-48], FEWZ [49-51], NNLOJET [11,12,52,53], and RESBOS [54,55]) and Monte Carlo (MC) integration error, see Sec. III B. Specifically, we have included the MC errors in the CT18(Z) analysis for the inclusive jet and high- $p_{T} Z$ boson production data. But, the PDF uncertainties related to the choice of the QCD scales and the codes for theoretical calculations have not been systematically included in this analysis. The important PDF parametrization uncertainty was investigated by repeating the fits for $\mathcal{O}(250)$ trial functional forms of the PDFs. (Our post-CT10 fits parametrize PDFs in terms of Bernstein polynomials, which simplify trying a wide range of parametrization forms to quantify/eliminate potential biases. Appendix C presents an example of such parametrization.) The final uncertainty on the nominal CT18 PDF set is determined so as to cover central solutions obtained with alternative parametrization forms and alternative fit settings or scale choices, see Sec. III C.

\section{B. Experimental datasets fitted in CT18}

The CT18 global analysis starts with the dataset baseline of CT14 HERAII [32] and adds the LHC results published before mid-2018. The experiments in the CT14 HERAII baseline are listed in Table I, while the new LHC datasets included in the CT18(Z) fit are shown in Table II. Tables I and II also include information on the number of data points, $\chi^{2}$, and the effective Gaussian variable, $S_{E}$, for each individual dataset appearing in the global fit. Most of the datasets are included in all four PDF ensembles; we will identify differences between the specific selections as they arise.

\section{Charting sensitivity of new datasets to the PDFs}

As discussed in Secs. II A 3 and II A 4, we employed a new method based on the Hessian sensitivity variables $[20,21]$ (informative descendants of the Hessian correlation between theoretical observables and PDFs $[22,90,91])$ to determine quantitatively a hierarchy of impact of data on the global fit, and on specific cross sections.

As a demonstration of this, the PDFSENSE framework can predict in advance which fitted datasets may have the most impact on one of the most crucial predictions at the LHC, such as the Higgs boson cross section $\left(\sigma_{H}\right)$ through the $g g$ fusion process (at $\sqrt{s}=14 \mathrm{TeV}$ ). Often, to get an indication which datasets will have the most impact on such cross sections, one examines the Pearson correlations [22,90,91] between the experimental data points and the gluon distribution in the kinematic region responsible for Higgs boson production. The left-hand side of Fig. 5 shows the data points with the highest absolute correlations $\left|C_{f}\right|$ 
TABLE I. Datasets included in the CT18(Z) NNLO global analyses. Here we directly compare the quality of fit found for CT18 NNLO vs CT18Z NNLO on the basis of $\chi_{E}^{2}, \chi_{E}^{2} / N_{p t, E}$, and $S_{E}$, in which $N_{p t, E}, \chi_{E}^{2}$ are the number of points and value of $\chi^{2}$ for experiment $E$ at the global minimum. $S_{E}$ is the effective Gaussian parameter $[38,42,56]$ quantifying agreement with each experiment. The ATLAS 7 TeV $35 \mathrm{pb}^{-1} \mathrm{~W} / \mathrm{Z}$ dataset, marked by $\neq$, is replaced by the updated one $\left(4.6 \mathrm{fb}^{-1}\right)$ in the CT18A and CT18Z fits. The CDHSW data, labeled by $\dagger$, are not included in the CT18Z fit. The numbers in parentheses are for the CT18Z NNLO fit.

\begin{tabular}{|c|c|c|c|c|c|c|}
\hline Exp. ID\# & Experimental dataset & & $N_{p t, E}$ & $\chi_{E}^{2}$ & $\chi_{E}^{2} / N_{p t, E}$ & $S_{E}$ \\
\hline 160 & $\begin{array}{l}\text { HERAI }+ \text { II } 1 \mathrm{fb}^{-1}, \mathrm{H} 1 \text { and ZEUS NC and } \\
\text { CC } e^{ \pm} p \text { reduced cross sec. comb. }\end{array}$ & {$[30]$} & 1120 & $1408(1378)$ & $1.3(1.2)$ & $5.7(5.1)$ \\
\hline 101 & BCDMS $F_{2}^{p}$ & {$[57]$} & 337 & $374(384)$ & $1.1(1.1)$ & $1.4(1.8)$ \\
\hline 102 & BCDMS $F_{2}^{d}$ & [58] & 250 & $280(287)$ & $1.1(1.1)$ & $1.3(1.6)$ \\
\hline 104 & $\mathrm{NMC} F_{2}^{d} / F_{2}^{p}$ & [59] & 123 & $126(116)$ & $1.0(0.9)$ & $0.2(-0.4)$ \\
\hline $108^{\dagger}$ & CDHSW $F_{2}^{p}$ & {$[60]$} & 85 & $85.6(86.8)$ & $1.0(1.0)$ & $0.1(0.2)$ \\
\hline $109^{\dagger}$ & CDHSW $x_{B} F_{3}^{p}$ & {$[60]$} & 96 & $86.5(85.6)$ & $0.9(0.9)$ & $-0.7(-0.7)$ \\
\hline 110 & CCFR $F_{2}^{p}$ & {$[61]$} & 69 & $78.8(76.0)$ & $1.1(1.1)$ & $0.9(0.6)$ \\
\hline 111 & CCFR $x_{B} F_{3}^{p}$ & [62] & 86 & $33.8(31.4)$ & $0.4(0.4)$ & $-5.2(-5.6)$ \\
\hline 124 & NuTeV $\nu \mu \mu$ SIDIS & {$[63]$} & 38 & $18.5(30.3)$ & $0.5(0.8)$ & $-2.7(-0.9)$ \\
\hline 125 & NuTeV $\bar{\nu} \mu \mu$ SIDIS & [63] & 33 & $38.5(56.7)$ & $1.2(1.7)$ & $0.7(2.5)$ \\
\hline 126 & CCFR $\nu \mu \mu$ SIDIS & {$[64]$} & 40 & $29.9(35.0)$ & $0.7(0.9)$ & $-1.1(-0.5)$ \\
\hline 127 & CCFR $\bar{\nu} \mu \mu$ SIDIS & {$[64]$} & 38 & $19.8(18.7)$ & $0.5(0.5)$ & $-2.5(-2.7)$ \\
\hline 145 & $\mathrm{H} 1 \sigma_{r}^{b}$ & {$[65]$} & 10 & $6.8(7.0)$ & $0.7(0.7)$ & $-0.6(-0.6)$ \\
\hline 147 & Combined HERA charm production & {$[66]$} & 47 & $58.3(56.4)$ & $1.2(1.2)$ & $1.1(1.0)$ \\
\hline 169 & $\mathrm{H} 1 F_{L}$ & [33] & 9 & $17.0(15.4)$ & $1.9(1.7)$ & $1.7(1.4)$ \\
\hline 201 & E605 Drell-Yan process & {$[67]$} & 119 & $103.4(102.4)$ & $0.9(0.9)$ & $-1.0(-1.1)$ \\
\hline 203 & E866 Drell-Yan process $\sigma_{p d} /\left(2 \sigma_{p p}\right)$ & {$[68]$} & 15 & $16.1(17.9)$ & $1.1(1.2)$ & $0.3(0.6)$ \\
\hline 204 & E866 Drell-Yan process $Q^{3} d^{2} \sigma_{p p} /\left(d Q d x_{F}\right)$ & [69] & 184 & $244(240)$ & $1.3(1.3)$ & $2.9(2.7)$ \\
\hline 225 & CDF run-1 lepton $A_{c h}, p_{T \ell}>25 \mathrm{GeV}$ & {$[70]$} & 11 & $9.0(9.3)$ & $0.8(0.8)$ & $-0.3(-0.2)$ \\
\hline 227 & CDF run-2 electron $A_{c h}, p_{T \ell}>25 \mathrm{GeV}$ & {$[71]$} & 11 & $13.5(13.4)$ & $1.2(1.2)$ & $0.6(0.6)$ \\
\hline 234 & $\mathrm{D} \varnothing$ run-2 muon $A_{c h}, p_{T \ell}>20 \mathrm{GeV}$ & [72] & 9 & $9.1(9.0)$ & $1.0(1.0)$ & $0.2(0.1)$ \\
\hline 260 & $\mathrm{D} \varnothing$ run- $2 Z$ rapidity & {$[73]$} & 28 & $16.9(18.7)$ & $0.6(0.7)$ & $-1.7(-1.3)$ \\
\hline 261 & CDF run- $2 Z$ rapidity & [74] & 29 & $48.7(61.1)$ & $1.7(2.1)$ & $2.2(3.3)$ \\
\hline 266 & CMS $7 \mathrm{TeV} 4.7 \mathrm{fb}^{-1}$, muon $A_{c h}, p_{T \ell}>35 \mathrm{GeV}$ & {$[75]$} & 11 & $7.9(12.2)$ & $0.7(1.1)$ & $-0.6(0.4)$ \\
\hline 267 & CMS $7 \mathrm{TeV} 840 \mathrm{pb}^{-1}$, electron $A_{c h}, p_{T \ell}>35 \mathrm{GeV}$ & [76] & 11 & $4.6(5.5)$ & $0.4(0.5)$ & $-1.6(-1.3)$ \\
\hline $268^{+\ddagger}$ & ATLAS $7 \mathrm{TeV} 35 \mathrm{pb}^{-1} W / Z$ cross sec., $A_{c h}$ & [77] & 41 & $44.4(50.6)$ & $1.1(1.2)$ & $0.4(1.1)$ \\
\hline 281 & $\mathrm{D} \varnothing$ run-2 $9.7 \mathrm{fb}^{-1}$ electron $A_{c h}, p_{T \ell}>25 \mathrm{GeV}$ & [78] & 13 & $22.8(20.5)$ & $1.8(1.6)$ & $1.7(1.4)$ \\
\hline 504 & CDF run-2 inclusive jet production & [79] & 72 & $122(117)$ & $1.7(1.6)$ & $3.5(3.2)$ \\
\hline 514 & $\mathrm{D} \varnothing$ run-2 inclusive jet production & {$[80]$} & 110 & $113.8(115.2)$ & $1.0(1.0)$ & $0.3(0.4)$ \\
\hline
\end{tabular}

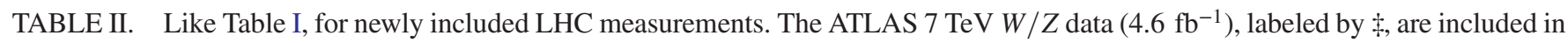
the CT18A and CT18Z global fits, but not in CT18 and CT18X.

\begin{tabular}{|c|c|c|c|c|c|c|}
\hline Exp. ID\# & Experimental dataset & & $N_{p t, E}$ & $\chi_{E}^{2}$ & $\chi_{E}^{2} / N_{p t, E}$ & $S_{E}$ \\
\hline 245 & LHCb $7 \mathrm{TeV} 1.0 \mathrm{fb}^{-1} \mathrm{~W} / Z$ forward rapidity cross sec. & [81] & 33 & $53.8(39.9)$ & $1.6(1.2)$ & $2.2(0.9)$ \\
\hline 246 & LHCb $8 \mathrm{TeV} 2.0 \mathrm{fb}^{-1} Z \rightarrow e^{-} e^{+}$forward rapidity cross sec. & [82] & 17 & $17.7(18.0)$ & $1.0(1.1)$ & $0.2(0.3)$ \\
\hline 248 & ATLAS $7 \mathrm{TeV} 4.6 \mathrm{fb}^{-1}, W / Z$ combined cross sec. & [39] & 34 & $287.3(88.7)$ & $8.4(2.6)$ & $13.7(4.8)$ \\
\hline 249 & CMS $8 \mathrm{TeV} 18.8 \mathrm{fb}^{-1}$ muon charge asymmetry $A_{c h}$ & [83] & 11 & $11.4(12.1)$ & $1.0(1.1)$ & $0.2(0.4)$ \\
\hline 250 & LHCb $8 \mathrm{TeV} 2.0 \mathrm{fb}^{-1} W / Z$ cross sec. & [84] & 34 & $73.7(59.4)$ & $2.1(1.7)$ & $3.7(2.6)$ \\
\hline 253 & ATLAS $8 \mathrm{TeV} 20.3 \mathrm{fb}^{-1}, Z p_{T}$ cross sec. & [85] & 27 & $30.2(28.3)$ & $1.1(1.0)$ & $0.5(0.3)$ \\
\hline 542 & $\begin{array}{l}\text { CMS } 7 \mathrm{TeV} 5 \mathrm{fb}^{-1} \text {, single incl. jet cross sec., } R=0.7 \\
\text { (extended in y) }\end{array}$ & [86] & 158 & $194.7(188.6)$ & $1.2(1.2)$ & $2.0(1.7)$ \\
\hline 544 & ATLAS $7 \mathrm{TeV} 4.5 \mathrm{fb}^{-1}$, single incl. jet cross sec., $R=0.6$ & [9] & 140 & $202.7(203.0)$ & $1.4(1.5)$ & $3.3(3.4)$ \\
\hline 545 & $\begin{array}{l}\text { CMS } 8 \mathrm{TeV} 19.7 \mathrm{fb}^{-1} \text {, single incl. jet cross sec., } R=0.7 \text {, } \\
\quad \text { (extended in y) }\end{array}$ & [87] & 185 & $210.3(207.6)$ & $1.1(1.1)$ & $1.3(1.2)$ \\
\hline 573 & $\begin{array}{l}\text { CMS } 8 \mathrm{TeV} 19.7 \mathrm{fb}^{-1}, t \bar{t} \text { norm. double-diff. top } p_{T} \text { and } y \\
\text { cross sec. }\end{array}$ & [88] & 16 & $18.9(19.1)$ & $1.2(1.2)$ & $0.6(0.6)$ \\
\hline 580 & ATLAS $8 \mathrm{TeV} 20.3 \mathrm{fb}^{-1}, t \bar{t} p_{T}^{t}$ and $m_{t \bar{t}}$ abs. spectrum & [89] & 15 & $9.4(10.7)$ & $0.6(0.7)$ & $-1.1(-0.8)$ \\
\hline
\end{tabular}




\section{$\left|C_{f}\right|$ for $\sigma_{H}(14 \mathrm{TeV})$, CT14HERA2}

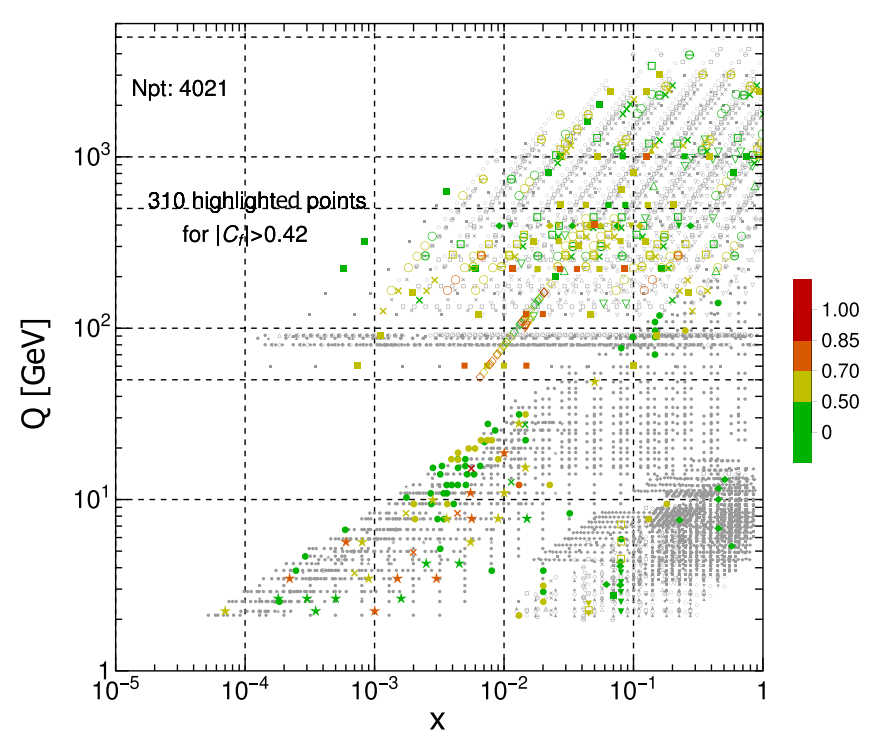

$\left|S_{f}\right|$ for $\sigma_{H}(14 \mathrm{TeV}), \mathrm{CT} 14 \mathrm{HERA} 2$

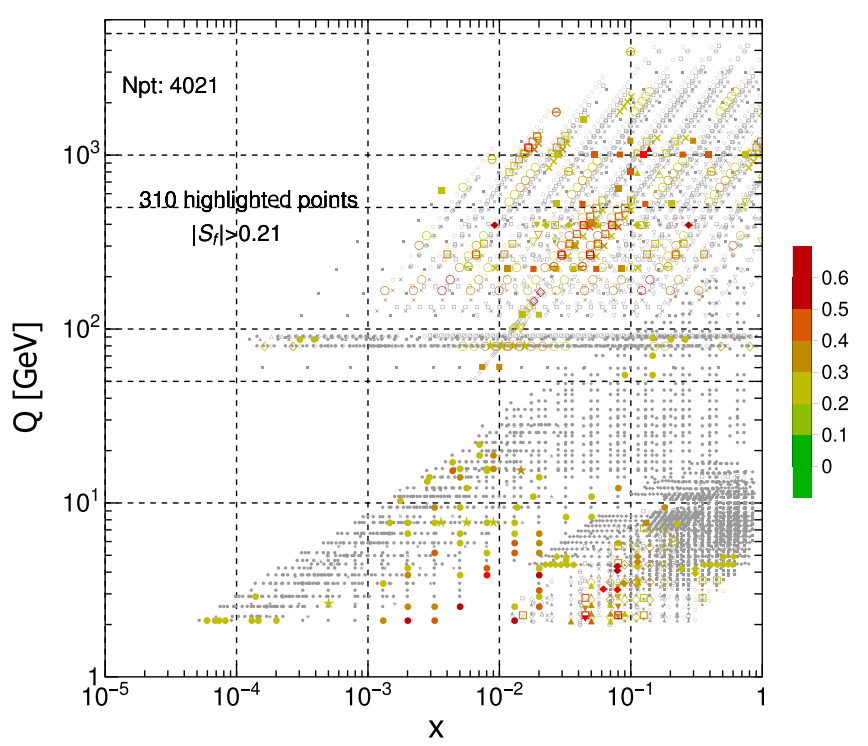

FIG. 5. Left: candidate data considered for inclusion in CT18 according to the magnitude of the Pearson correlation $C_{f}$ between the total Higgs cross section at $14 \mathrm{TeV}, \sigma_{H}(14 \mathrm{TeV})$ and the residual of each point as determined within the PDFSENSE framework [20]. Right: a similar assessment of the CT18 candidate data, but computed on the basis of the sensitivity, $\left|S_{f}\right|\left(x_{i}, Q_{i}\right)$. In both panels, a highlighting cut has been imposed to draw attention to the $~ 300$ highest-impact points according to each metric.

(defined by the statistical residuals as in Ref. [20]) with the Higgs boson cross section at $14 \mathrm{TeV}$.

By this measure, there may be a number of high-impact datasets, notably HERA neutral current DIS, LHC and Tevatron jet production, and HERA charm quark production. The correlations, however, do not reflect the experimental uncertainties of the data points: an experimental cross section could be highly correlated with the gluon distribution in the $x$ range responsible for Higgs boson production and still not provide much of a constraint on the Higgs boson cross section if the experimental uncertainties are large. Conversely, an experimental cross section that might not have as large a correlation, but which has smaller (statistical and systematic) uncertainties, may provide a stronger constraint.

The level of constraint is thus better predicted by the sensitivity variable $S_{f}$, defined in Ref. [20]. The experimental data points used in the CT18 global fit that have the highest absolute sensitivity $\left|S_{f}\right|$ to the PDF dependence of $\sigma_{H}$ at $14 \mathrm{TeV}$ are shown on the right-hand side of Fig. 5. More data points (from a larger number of experiments) have high sensitivity than those identified by high correlation. In addition to the DIS data from HERA I + II, there are also contributions from the fixed-target DIS experiments, as well as measurements from the LHC.

As will be shown in Sec. VA 2 using Lagrange multiplier scans, the HERA I + II dataset, with its abundant data points and small experimental errors, still dominates the constraints on the gluon distribution in the range sensitive to Higgs boson production at the LHC. Because of the continuing influence of the older datasets, we will find that the reduction of the PDF uncertainty for Higgs boson production is less significant in CT18 than in CT14. In addition, tension between some of the most sensitive datasets limits the reduction on the uncertainty of the Higgs cross section. These effects are explored in detail in Sec. VA 2.

We will now discuss the new datasets included in the CT18 analysis, and highlight the differences in the alternative fits.

\section{Baseline datasets}

The CT18 global analysis inherits from CT14 ${ }_{\text {HERAII }}$ a number of precision non-LHC experiments listed in Table I. Among those, the HERA I + II DIS dataset provides the most significant constraints, followed by a group of fixedtarget neutral-current DIS experiments: BCDMS, NMC, and CCFR. Similarly, a number of neutrino DIS measurements have previously been included and provide valuable constraints on sea (anti)quarks. Among them, we find that the single-nucleon structure functions $F_{2}^{p}$ and $F_{3}^{p}$ extracted from CDHSW data on neutrino-iron deep inelastic scattering exhibit a preference for a harder gluon PDF at $x \gtrsim 0.1$, compared to CCFR and other experiments, cf. Fig. 21. This well-known behavior reflects larger logarithmic slopes of $F_{2}^{p}$ and $x F_{3}^{p}$ measured by CDHSW, as compared to the analogous CCFR measurements [92], which in turn may reflect differences in the energy calibration and resolution smearing between the two experiments [93]. Thus, to help 
obtain a softer large- $x$ gluon behavior, as being favored by recent LHC data, we exclude the CDHSW $F_{2}$ and $x_{B} F_{3}$ datasets from the CT18Z analysis, while including these sets in the rest of the CT18 PDF ensembles.

We continue to include a variety of lepton pair production measurements from the Tevatron and fixed-target experiments, as summarized in Table I. The low-statistics data on $W / Z$ production at $\mathrm{LHCb} 7 \mathrm{TeV}$ [94] and ATLAS, CMS $7 \mathrm{TeV}$ jet production $[95,96]$ are replaced in the CT18 analyses by more recent measurements, as summarized in the next section.

\section{LHC precision data from $W / Z$ vector boson production}

The CT18(Z) global analysis uses $W / Z$ vector boson production data from LHC run-I, including measurements from the ATLAS, CMS, and LHCb collaborations.

The measurements from ATLAS included in the fit are these:

(1) The $\sqrt{s}=7 \mathrm{TeV} W / Z$ combined cross section measurements [39] $\left(\right.$ Exp. ID = 248) with $4.6 \mathrm{fb}^{-1}$ of integrated luminosity. The ATLAS group has performed seven measurements with a total of 61 data points: distributions in the pseudorapidity of charged lepton in $W^{+}$(11 points) and $W^{-}$(11 points) production, rapidity of lepton pairs for low-mass Drell-Yan (DY) process in the central region (6 points), Z-peak DY process in the central (12 points) and forward ( 9 points) regions, and high-mass DY process in the central (6 points) and forward (6 points) regions. In the published fits, we include three measurements: $W^{+}, W^{-}$, and $Z$-peak central DY production (34 points in total). These data are used only to fit the CT18A and CT18Z PDFs, but not the CT18 and CT18X PDFs. Other data are ignored due to the sizable EW corrections and/or photon-induced contribution $\left(\gamma \gamma \rightarrow l^{+} l^{-}\right)$, as discussed in Sec. V C.

(2) The $\sqrt{s}=8 \mathrm{TeV}$ distribution of transverse momentum $p_{T}$ of lepton pairs in the $Z / \gamma^{*}$ production (Exp. $\mathrm{ID}=253$ ) [85] with $20.3 \mathrm{fb}^{-1}$ of integrated luminosity. The ATLAS collaboration measured the $p_{T, t \bar{e}}$ distribution up to $900 \mathrm{GeV}$ for the lepton pairs in the invariant mass range $12<M_{\ell \bar{\ell}}<150 \mathrm{GeV}$. Meanwhile, the experimentalists presented both the normalized and absolute cross sections for the singly differential distribution $d \sigma / d p_{T, \ell \bar{\ell}}$ and doubly differential distribution $d^{2} \sigma /\left(d p_{T, \ell \bar{\ell}} d y_{\ell \bar{\ell}}\right)$. To select the cleanest and most sensitive data for the CT18 fits, we only include three invariant-mass bins around the Z-peak region: $M_{\ell \bar{\ell}} \in[46-66,66-116$, $116-150] \mathrm{GeV}$. We do not include the data at $M_{\ell \bar{\ell}}<46 \mathrm{GeV}$, for which the kinematic cut $p_{T}^{l}>$ $20 \mathrm{GeV}$ restricts the cross section to be coming predominantly from the region $p_{T, \ell \bar{\ell}} \gtrsim M_{\ell \bar{\ell}}$, where the higher-order corrections beyond the current $\mathcal{O}\left(\alpha_{s}^{3}\right)$ calculation are significant. We fit neither the normalized $p_{T, \ell \bar{\ell}}$ distributions, as they introduce artificial interdependence between the particle rates in the disparate $p_{T, \ell \bar{\ell}}$ regions through their shared overall normalization, nor the doubly differential distributions, which are not very sensitive. In total, we include 27 data points in the pair's transverse momentum region $45 \leq p_{T, \ell \bar{\ell}} \leq 150 \mathrm{GeV}$, where the fixed-order NNLO cross section is most reliable. The data at lower $p_{T, \ell \bar{\ell}}$ and higher $p_{T, \ell \bar{\ell}}$ regions are excluded because of contributions from small- $p_{T}$ resummation and electroweak corrections, as discussed in Sec. V C.

For CMS, measurements of the charge asymmetry for inclusive $W^{ \pm}$production at $\sqrt{s}=8 \mathrm{TeV}$ [83] (Exp. $\mathrm{ID}=249$ ) are included, with $18.8 \mathrm{fb}^{-1}$ of integrated luminosity. These consist of 11 bins of muon pseudorapidity (over the range $0 \leq\left|\eta^{\mu}\right| \leq 2.4$ ) with $p_{T}^{\mu} \geq 25 \mathrm{GeV}$. The correlated systematic errors are implemented using a decomposition of the covariance matrix to convert it to the correlation matrix representation according to the procedure described in Appendix B. The same decomposition method will also be applied to the LHCb $W / Z$ experiments (Exp. ID 245 and 250) to convert the published covariance matrix to a correlation matrix. We have explicitly verified their equivalence using EPUMP [25], capable of operating with both the covariance matrix and correlation matrix representations.

In CT18, we include three experimental datasets published by LHCb:

(1) The $\sqrt{s}=7 \mathrm{TeV} W / Z$ forward rapidity cross section measurements [81] (Exp. ID = 245), with $1.0 \mathrm{fb}^{-1}$ of integrated luminosity, consist of 17 bins of $Z$-boson rapidity $\left(2.0 \leq y_{Z} \leq 4.25\right)$ for $Z$ boson production cross sections and eight bins of muon pseudorapidity $\left(2.0 \leq \eta^{\mu} \leq 4.5\right)$ for $W^{+}$or $W^{-}$ boson productions. Similarly to the CMS charge asymmetry measurements discussed above, the systematic errors are included by converting the published covariance matrix into a correlation matrix. The beam energy and luminosity uncertainties are taken to be fully correlated between the crosssection measurements. This dataset replaces previous LHCb measurements [94] of inclusive vector boson production and lepton-charge asymmetry in the forward region, with $35 \mathrm{pb}^{-1}$ of integrated luminosity.

(2) The $\sqrt{s}=8 \mathrm{TeV} \mathrm{Z} \rightarrow e^{+} e^{-}$cross section measurements [82] $(\mathrm{ID}=246)$ at forward rapidity, with $2.0 \mathrm{fb}^{-1}$ of integrated luminosity, consist of 17 bins of $Z$-boson rapidity $\left(2.0 \leq y_{Z} \leq 4.25\right)$. The luminosity uncertainty is taken to be fully correlated, but the other correlated uncertainties are simply added in quadrature. We have used EPUMP to confirm that this 
approximation yields similar updated PDFs as those obtained from using the covariance matrix representation.

(3) The $\sqrt{s}=8 \mathrm{TeV} W / Z$ production cross section measurements [84] (Exp. ID = 250), with $2.0 \mathrm{fb}^{-1}$ of integrated luminosity, consist of 18 bins of $Z$-boson rapidity $\left(2.0 \leq y_{Z} \leq 4.5\right)$ and eight bins of muon pseudorapidity $\left(2.0 \leq \eta^{\mu} \leq 4.5\right)$ for $W^{+}$or $W^{-}$boson productions. As in the $\sqrt{s}=7 \mathrm{TeV}$ case, the correlated systematic errors are included by converting the covariance matrix into the correlation matrix representation. The beam energy and luminosity uncertainties are taken to be fully correlated between the cross section measurements.

\section{LHC inclusive jet production}

For CMS, double-differential cross section measurements, $d^{2} \sigma /\left(d p_{T} d y\right)$, for jet production at both $\sqrt{s}=7$ and $8 \mathrm{TeV}$, are used. We use the larger of the two jet radii ( $R=0.7$ ) [86]. The datasets consist of $5 \mathrm{fb}^{-1}$ of integrated luminosity at $\sqrt{s}=7 \mathrm{TeV}$ (Exp. ID $=542)$, and $19.7 \mathrm{fb}^{-1}$ at $\sqrt{s}=8 \mathrm{TeV}$ [87] $($ Exp. ID =545). The $7 \mathrm{TeV}$ jet measurement contains 158 data points in six bins of rapidity (with total rapidity coverage of $0 \leq|y| \leq 3.0$ ), covering the jet transverse momentum range $56 \leq p_{T} \leq 1327 \mathrm{GeV}$. At $8 \mathrm{TeV}$, six rapidity bins of jet data are also used, covering the rapidity range $0 \leq|y| \leq 3.0$. There are a total of 185 data points, with a transverse momentum range of $74 \leq p_{T} \leq 2500 \mathrm{GeV}$. These new CMS measurements replace the previous ones published in Ref. [96] with $5 \mathrm{pb}^{-1}$ of integrated luminosity.

In addition to the systematic error information provided in the HEPDATA files, jet energy corrections (JEC) in the CMS $7 \mathrm{TeV}$ data have been decorrelated according to the procedure in Ref. [97]. In particular the JEC2 ("e05") and an additional CMS-advocated decorrelation for $|y|>2.5$, have been implemented [98]. These decorrelations improve the ability to fit the data.

For ATLAS, we again use the larger of the two jet radii $(R=0.6)$. Inclusive jet cross section measurements at $\sqrt{s}=7 \mathrm{TeV}$ with $R=0.6$ and $4.5 \mathrm{fb}^{-1}$ of integrated luminosity [9] $($ Exp. ID = 544) are included in the global fit. This dataset contains six bins covering the rapidity range $0.0 \leq y \leq 3.0$, with a total of 140 data points in the $74 \leq p_{T} \leq 1992 \mathrm{GeV}$ range. This dataset replaces the previous dataset [95] which contains $37 \mathrm{pb}^{-1}$ data.

Following the prescription given in Ref. [10], two jet energy scale uncertainties have been decorrelated in the ATLAS $7 \mathrm{TeV}$ jet data, namely, MJB (fragmentation) ("jes16"), and flavor response ("jes62"). The decorrelation procedure reduces the $\chi^{2}$ value by approximately 92 units. The total contribution to the $\chi^{2}$ from the systematic error shifts is 28 (for 74 correlated systematic errors). Only one of the systematic error sources requires a shift greater than 2 standard deviations. A further improvement of 52 units is obtained by including a $0.5 \%$ theoretical error to account for statistical noise associated with the Monte Carlo calculations of the needed NNLO/NLO $K$ factors [11-13] in the NNLO fit, as detailed in Sec. III B 1. More details on the treatment of the ATLAS inclusive jet data are provided in Appendix E.

\section{LHC top-quark pair production}

ATLAS and CMS have measured top-quark pair production differential cross sections as a function of the top(anti)quark transverse momentum $p_{T, t}$, invariant mass $m_{t \bar{t}}$, rapidity of the pair $y_{t \bar{t}}$, transverse momentum of the topquark pair $p_{T, t \bar{t}}$, and top-quark rapidity $y_{t}$, individually for ATLAS, and in pairs for CMS. The individual impacts of the single differential $t \bar{t}$ cross section measurements have been analyzed, first by using the PDFSENSE sensitivity framework of Ref. [20], and second in separate fits via EPUMP in Refs. $[25,99]$. There is some tension between the $t \bar{t}$ observables that leads to different pulls on the gluon distribution that each prefers. Difficulties in fitting simultaneously $p_{T, t}, m_{t \bar{t}}, y_{t}$, and $y_{t \bar{t}}$ distributions at $8 \mathrm{TeV}$ were also found in $[25,99,100]$.

For the CT18 analysis, we thus decided to select a few top-quark production measurements with the best compatibility within the fit. In the case of ATLAS, more than one $t \bar{t}$ observable can be included by making use of their published statistical correlations. We have chosen the absolute differential cross sections $d \sigma / d p_{T, t}$ for the top$p_{T}$ and $d \sigma / d m_{t \bar{t}}$ for the invariant mass, at $\sqrt{s}=8 \mathrm{TeV}$ with $20.3 \mathrm{fb}^{-1}$ of integrated luminosity $($ Exp. ID $=580$ ) [89], based on the recommendation from ATLAS. ${ }^{3}$

The two ATLAS measurements are combined into one single dataset, which includes the full phase-space absolute differential cross sections after the combination of the $e+$ jets and $\mu+$ jets channels for the $p_{T, t}$ and $m_{t \bar{t}}$ distributions with statistical correlations. Both of these distributions are fitted together by decorrelating one of the systematic uncertainties relative to the parton shower [101]. The QCD theoretical predictions at NNLO for these observables are obtained by using fastNNLO tables provided in Refs. $[17,18]$.

In an upcoming study, we find that the ATLAS rapidity distributions of a single quark and top pair, $y_{t}$ and $y_{t \bar{t}}$, can be fitted in the CT18 setup with $\chi_{E}^{2} / N_{p t, E}>2.3$-too high for the fit to be acceptable, which is consistent with the findings in Ref. [102]. These distributions show tensions with some other datasets. Their inclusion, either in the single-differential or double-differential form, would not lead to the reduction of the PDF uncertainty.

\footnotetext{
${ }^{3}$ A. Cooper-Sarkar, private communication, and ATLASPHYS-PUB-2018-017.
} 
For CMS, we have chosen the normalized double differential cross section $d^{2} \sigma / d p_{T, t} d y_{t}$ at $\sqrt{s}=8 \mathrm{TeV}$, with $19.7 \mathrm{fb}^{-1}$ (Exp. ID = 573) [88].

The observed effect of the $t \bar{t}$ datasets on the CT18 PDFs is modest, when they are included together with the Tevatron and LHC jet production. Their impact on the gluon PDF is compatible with the jet data, but the jet data provide stronger constraints due to their larger numbers of data points, wider kinematic range, and relatively small statistical and systematic errors.

In the course of the CT18 analysis, CMS measurements of top-quark pair production differential cross sections at $13 \mathrm{TeV}$ were published [103], and bin-by-bin data correlations were made available on the HEPDATA repository. While these measurements are not currently included in the CT18 global fit, their description is discussed later in Sec. VI.

\section{Other LHC measurements not included in the CT18 fits}

Besides the CT18(Z) data ensemble, we have carefully investigated several other high-luminosity measurements from LHC run-I. In certain cases we observed either no significant impact or substantial tensions with the CT18(Z) baseline. The following vector boson production data were examined using PDFSENSE, EPUMP, or full fits, but not included in the final CT18(Z) global analysis:

(1) Difficulties were encountered in obtaining a good agreement between theory and the ATLAS $\sqrt{s}=$ $7 \mathrm{TeV} Z$-boson transverse momentum distribution $\left(p_{T, \ell \bar{\ell}}\right)$ data with $4.7 \mathrm{fb}^{-1}$ of integrated luminosity [104]. The subset of these data with $p_{T, \ell \bar{\ell}} \sim M_{\ell \bar{\ell}}$ (in the kinematic region most amenable to a fixed-order calculation) has rendered unacceptably high $\chi^{2}$ values for various combinations of the renormalization and factorization scales that we have tried. No significant constraints could be ascribed to the CMS $\sqrt{s}=8 \mathrm{TeV} p_{T, \ell \bar{\ell}}$ and $y_{\ell \bar{\ell}}$ distributions with $19.7 \mathrm{fb}^{-1}$ of integrated luminosity [105] in the $Z$ peak kinematic region, and to the CMS $\sqrt{s}=8 \mathrm{TeV}$ normalized $W p_{T}$ and $Z p_{T}$ spectra with $18.4 \mathrm{pb}^{-1}$ [106]. When comparing to the CMS double-differential distributions in $\left(p_{T, \ell \bar{\ell}}, y_{\ell \bar{\ell}}\right)$, we observed a large discrepancy between theory and data in the last rapidity bin. For the normalized $p_{T, \ell \bar{\ell}}$ distributions of lepton pairs presented by both ATLAS and CMS groups, it was not clear how to consistently compare to data in a limited range $p_{T, \ell \bar{\ell}} \sim M_{\ell \bar{\ell}}$ when the normalization of data points was dependent on the cross section outside of the fitted range.

(2) No substantial changes in the candidate PDFs were observed after including either the single- or doubledifferential distributions, $d \sigma / d Q$ or $d^{2} \sigma /(d Q d y)$, of the ATLAS $\sqrt{s}=8 \mathrm{TeV}$ Drell-Yan cross section measurements at $116 \leq Q \leq 1500 \mathrm{GeV}$ and $0 \leq y_{Z} \leq 2.5$ with $20.3 \mathrm{fb}^{-1}$ of integrated luminosity [107]. These high-mass data are impacted by non-negligible EW corrections and photon-induced (PI) dilepton production, the point that is further addressed in Sec. V C. For the same reason, we do not include the data of ATLAS $7 \mathrm{TeV}$ high-mass Drell-Yan production with $4.7 \mathrm{fb}^{-1}$ of integrated luminosity [108].

(3) The low-mass Drell-Yan data by the ATLAS collaboration at $7 \mathrm{TeV}$ [109] were also explored and found to have no significant impact on the PDFs.

(4) We have explored the impact of the data of $W$-boson associated with charm-jet production from ATLAS [110] and CMS [111] measurements at $7 \mathrm{TeV}$. As the NNLO calculations for $W+$ charm jet are not available, we use these data only to compare against the NLO theoretical predictions in Sec. VIC.

(5) No significant impact is found by including the single- or double-differential cross sections, $d \sigma / d Q$ or $d^{2} \sigma /(d Q d y)$, of the CMS DY data taken at 7 [112] and 8 [113] TeV. These data are not included in the CT18 fits for the following reasons. First, the EW corrections and photon-induced contributions to these data are non-negligible in the high-mass region. Second, these data are presented as cross sections over the full phase space, a fact that introduces additional uncertainties from the unfolding procedure. The $8 \mathrm{TeV}$ dataset has $\chi_{E}^{2} / N_{p t, E} \approx 2$ for CT18(Z) PDFs and does not modify the PDFs when examined using EPUMP and PDFSENSE.

\section{Alternative PDF fits: CT18A, CT18X, CT18Z}

We are now ready to review the three additional fits that were explored in parallel with CT18 by making alternative choices for data selection and theoretical calculations. The key differences among these fits are listed in Table III. Their predictions will be compared in Appendix A.

(i) CT18X differs from CT18 in adopting an alternate scale choice for the DIS datasets. It is most common to compute the inclusive DIS cross sections using the photon's virtuality as the factorization scale, $\mu_{F, D I S}^{2}=$ $Q^{2}$. It has been argued, however, that resummation of logarithms $\ln ^{p}(1 / x)$ at $x \ll 1$ improves agreement with HERA run I + II data by several tens of units of $\chi^{2}[34,35]$. In our analysis, we observe that, by evaluating the DIS cross sections in a fixed-order calculation at NNLO accuracy, with a tuned factorization scale $\mu_{F, x}^{2} \equiv 0.8^{2}\left(Q^{2}+0.3 \mathrm{GeV}^{2} / x_{B}^{0.3}\right)$, instead of the conventional $\mu_{F}^{2}=Q^{2}$, we achieve nearly the same quality of improvement in the description of the HERA DIS dataset as in the analyses with low- $x$ resummation $[34,35]$. The fit done with these modified settings is designated as CT18X. For this fit, the $\chi^{2}$ of HERA I + II reduces by 
TABLE III. A summary of theoretical settings and dataset choices in CT18 and each of the three alternative fits: CT18A, CT18X, and CT18Z. The lattermost of these is compared with CT18 throughout the main text of this article, whereas more detail regarding each of the alternative fits is presented in Appendix A.

\begin{tabular}{|c|c|c|c|c|}
\hline PDF ensemble & Factorization scale in DIS & $\begin{array}{c}\text { ATLAS } 7 \mathrm{TeV} W / Z \\
\text { data included? }\end{array}$ & $\begin{array}{l}\text { CDHSW } F_{2}^{p, d} \\
\text { data included? }\end{array}$ & $\begin{array}{l}\text { Pole charm } \\
\text { mass, GeV }\end{array}$ \\
\hline CT18 & $\mu_{F, D I S}^{2}=Q^{2}$ & No & Yes & 1.3 \\
\hline CT18A & $\mu_{F, D I S}^{2}=Q^{2}$ & Yes & Yes & 1.3 \\
\hline CT18X & $\mu_{F, D I S}^{2}=0.8^{2}\left(Q^{2}+\frac{0.3 \mathrm{GeV}^{2}}{x_{B}^{0.3}}\right)$ & No & Yes & 1.3 \\
\hline CT18Z & $\mu_{F, D I S}^{2}=0.8^{2}\left(Q^{2}+\frac{0.3 \mathrm{GeV}^{2}}{x_{B}^{0.3}}\right)$ & Yes & No & 1.4 \\
\hline
\end{tabular}

more than 50 units in the kinematical region with $Q>2 \mathrm{GeV}$ and $x>10^{-5}$, assessed in the CT18 global fit. The CT18X prediction for $\mathrm{H} 1 F_{L}$ is moderately higher than that for CT18, which improves $\chi_{E}^{2}$ for $\mathrm{H} 1 F_{L}$ by a few units. See an illustration in Fig. 3 and its discussion in the Executive Summary II A 2.

The parametric form of the $x_{B}$-dependent scale $\mu_{F, x}^{2}$ is inspired by saturation arguments (see, e.g., $[114,115])$. The numerical coefficients in $\mu_{F, x}^{2}$ are chosen to minimize $\chi^{2}$ for the HERA DIS data. At $x \gtrsim 0.01, \mu_{F, x}^{2} \approx 0.8 Q^{2}$ results in larger NNLO DIS cross sections than with $\mu_{F}^{2}=Q^{2}$, as it might happen due to contributions from next-to-NNLO (N3LO) and beyond. At $x \lesssim 0.01, \mu_{F, x}^{2}$ numerically reduces the $Q^{2}$-derivative of NNLO DIS cross sections. In turn, these changes result in the enhanced gluon PDF at small $x$ and reduced gluon at $2.5 \times 10^{-2} \lesssim x \lesssim 0.2$.

(ii) Unlike CT18, the CT18A analysis includes high-luminosity ATLAS $7 \mathrm{TeV} W / Z$ rapidity distributions [39] that show some tension with DIS experiments and prefer a larger strangeness PDF than the DIS experiments in the small $x_{B}$ region. Inclusion of the ATLAS $7 \mathrm{TeV} W / Z$ data leads to significant deterioration in the $\chi_{E}^{2}$ values (i.e., larger $S_{E}$ values) for the dimuon SIDIS production data (NuTeV, CCFR), which are strongly sensitive to the strangeness PDF. One way to see this is to compare the $S_{E}$ distributions for the CT18 fit in Fig. 4 and the counterpart figure for CT18Z in Fig. 59 of Appendix A. The comparison shows that the $S_{E}$ values for CCFR and $\mathrm{NuTeV}$ dimuon datasets are elevated in the CT18Z fit, as compared to the CT18 fit, as a consequence of inclusion of the ATLAS $W / Z$ data in the CT18Z fit. Another way to see this was carried out in Ref. [25], using the EPUMP program.

(iii) CT18Z represents the accumulation of these settings introduced to obtain a PDF set that is maximally different from CT18, despite achieving about the same global $\chi^{2} / N_{p t}$ as CT18. The CT18Z fit includes the $7 \mathrm{TeV} W / Z$ production data of ATLAS like CT18A, but it also includes the modified DIS scale choice, $\mu_{F, x}$, as done for CT18X. In addition to these modifications, CT18Z excludes the CDHSW extractions of the $F_{2}$ and $x_{B} F_{3}$ structure functions from $\nu \mathrm{Fe}$ scattering, which otherwise would oppose the trend of CT18Z to have a softer gluon at $x>0.1$, cf. Sec. II B 2. Finally, CT18Z is done by assuming a slightly higher value of the charm quark mass ( $1.4 \mathrm{GeV}$ compared to $1.3 \mathrm{GeV}$ ) in order to modestly improve the fit to the vector boson production data.

The combination of these choices in the CT18Z analysis results in a Higgs boson production cross section via gluon fusion that is reduced by about $1 \%$ compared to the corresponding CT14 and CT18 predictions. Thus, the various choices made during the generation of four CT18(A,X,Z) fits allow us to more faithfully explore the full range of the PDF behavior at NNLO that is consistent with the available hadronic data, with implications for electroweak precision physics.

\section{THEORETICAL INPUTS TO CT18}

Modern global fits determine the PDFs from a large number of data points ( $N_{p t}>3600$ for CT18), provided by a wide variety of experimental measurements (39 datasets for CT18), and involving thousands of iterations of multivariate fits, with the theoretical cross sections evaluated at NNLO. In the CT18 fits, the $x$ dependence of the input PDFs, at the initial scale $Q_{0}$ equal to the pole mass of the charm quark, is parametrized by Bernstein polynomials, multiplied by the standard $x^{a}$ and $(1-x)^{b}$ factors that determine the small- $x$ and large- $x$ asymptotics. In these functions, there are five to eight independent fitting parameters for each parton flavor except strangeness; additional parameters may be determined by momentum and flavor sum rules or (if poorly constrained) fixed at physically reasonable values.

In the present section, we review the essential components of our theoretical setup: the goodness-of-fit function in Sec. III A, computer programs for (N)NLO computations for various processes in Sec. III B, and input parametric forms for the PDFs in Sec. III C. The explicit parametric 
forms for the best-fit CT18 PDFs are presented in Appendix C.

\section{A. Goodness of fit function and the covariance matrix}

The CTEQ-TEA analyses quantify the goodness-of-fit to an experimental dataset $E$ with $N_{p t}$ data values by means of the log-likelihood function [23],

$\chi_{E}^{2}(a, \lambda)=\sum_{k=1}^{N_{p t}} \frac{1}{s_{k}^{2}}\left(D_{k}-T_{k}(a)-\sum_{\alpha=1}^{N_{\lambda}} \lambda_{\alpha} \beta_{k \alpha}\right)^{2}+\sum_{\alpha=1}^{N_{\lambda}} \lambda_{\alpha}^{2}$.

A $k$ th datum is typically provided as a central value $D_{k}$, an uncorrelated statistical error $s_{k, \text { stat }}$, and

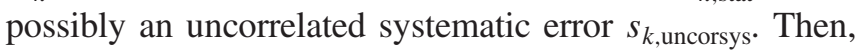
$s_{k} \equiv \sqrt{s_{k, \text { stat }}^{2}+s_{k \text {,uncorsys }}^{2}}$ is the total uncorrelated error on the measurement $D_{k}$.

$T_{k}$ is the corresponding theory value that depends on the PDF parameters $\left\{a_{1}, a_{2}, \ldots\right\} \equiv a$. In addition, the $k$ th datum may depend on $N_{\lambda}$ correlated systematic uncertainties, and those may be fully correlated over all data points. To estimate such errors, it is common to associate each source of the correlated error with an independent random nuisance parameter $\lambda_{\alpha}$ that is assumed to be sampled from a standard normal distribution, unless known otherwise. The experiment does not tell us the values of $\lambda_{\alpha}$ but it may provide the change $\beta_{k \alpha} \lambda_{\alpha}$ of $D_{k}$ under a variation of $\lambda_{\alpha}$. Knowing $\beta_{k \alpha}$, one can estimate the likely values of $\lambda_{\alpha}$, as well as the uncertainty in the PDF parameters for a plausible range of $\lambda_{\alpha}$.

For those experiments $E$ that provide $\beta_{k \alpha}$, we find that, at the global minimum $a_{0}$, the best-fit $\chi^{2}$ value is given as

$$
\chi_{E}^{2}\left(a_{0}, \bar{\lambda}\left(a_{0}\right)\right)=\sum_{i=1}^{N_{p t}} r_{i}^{2}\left(a_{0}\right)+\sum_{\alpha=1}^{N_{\lambda}} \bar{\lambda}_{\alpha}^{2}\left(a_{0}\right)
$$

in terms of the best-fit shifted residuals,

$$
r_{i}\left(a_{0}\right)=s_{i} \sum_{j=1}^{N_{p t}}\left(\operatorname{cov}^{-1}\right)_{i j}\left(D_{j}-T_{j}\left(a_{0}\right)\right),
$$

and best-fit nuisance parameters,

$$
\bar{\lambda}_{\alpha}\left(a_{0}\right)=\sum_{i, j=1}^{N_{p t}}\left(\operatorname{cov}^{-1}\right)_{i j} \frac{\beta_{i \alpha}}{s_{i}} \frac{\left(D_{j}-T_{j}\left(a_{0}\right)\right)}{s_{j}},
$$

where

$$
\left(\operatorname{cov}^{-1}\right)_{i j}=\left[\frac{\delta_{i j}}{s_{i}^{2}}-\sum_{\alpha, \beta=1}^{N_{\lambda}} \frac{\beta_{i \alpha}}{s_{i}^{2}} A_{\alpha \beta}^{-1} \frac{\beta_{j \beta}}{s_{j}^{2}}\right]
$$

and

$$
A_{\alpha \beta}=\delta_{\alpha \beta}+\sum_{k=1}^{N_{p t}} \frac{\beta_{k \alpha} \beta_{k \beta}}{s_{k}^{2}} .
$$

These relations are derived in Appendix B.
Another instructive form expresses $r_{i}\left(a_{0}\right)$ in terms of the shifted data values, $D_{i}^{s h} \equiv D_{i}-\sum_{\alpha=1}^{N_{\lambda}} \bar{\lambda}_{\alpha}\left(a_{0}\right) \beta_{k \alpha}$ :

$$
r_{i}\left(a_{0}\right)=\frac{D_{i}^{s h}\left(a_{0}\right)-T_{i}\left(a_{0}\right)}{s_{i}} .
$$

Sometimes, we take extra steps to convert the published table of correlated uncertainties into the $\beta_{k \alpha}$ matrix formatted in accord with Eq. (1). For example, when an experiment distinguishes between positive and negative systematic variations, we average these for each data point for consistency with the normally distributed $\lambda_{\alpha}$. (We have verified that the choice of the averaging procedure does not significantly affect the outcomes, e.g., if a central value is shifted to be in the middle of an originally asymmetric interval, etc.)

In a small number of experimental publications, only a form based on the covariance matrix $(\mathrm{cov})_{i j}$ is used in place of Eq. (1):

$$
\chi_{E}^{2}(a)=\sum_{i, j=1}^{N_{p t}}\left(\operatorname{cov}^{-1}\right)_{i j}\left(D_{i}-T_{i}(a)\right)\left(D_{j}-T_{j}(a)\right) .
$$

While we can compute $\chi^{2}$ directly using Eq. (8), when deriving the PDFs, we find it convenient to go back to the form consisting of the uncorrelated errors $s_{i}$ and the correlated contributions provided by $\beta_{k \alpha}$ :

$$
(\mathrm{cov})_{i j} \approx s_{i}^{2} \delta_{i j}+\sum_{\alpha=1}^{N_{\lambda}} \beta_{i \alpha} \beta_{j \alpha} .
$$

An algorithm to construct such a representation with sufficient accuracy is presented at the end of Appendix B. In all relevant cases, we have checked that both the input covariance matrix (cov) $)_{i j}$ and its decomposed version (9) produce close values of $\chi^{2}$. With the latter representation, we are also able to examine the shifted data values and shifted residuals, Eq. (7), to explore agreement with the individual data points.

In this article, we generally follow the CTEQ methodology and obtain $r_{i}\left(a_{0}\right)$ directly from the CTEQ-TEA fitting program, together with the optimal nuisance parameters $\bar{\lambda}_{\alpha}\left(a_{0}\right)$ and shifted central data values $D_{i}^{s h}(a)$.

\section{B. Theoretical computations and programs}

\section{Overview}

For deep-inelastic scattering observables, we perform computations using an NNLO realization [116] of the SACOT- $\chi$ heavy-quark scheme [117-120] adopted since CT10 NNLO [42]. These can be done using either the pole or $\overline{\mathrm{MS}}$ quark masses as the input [121], with the default choices of quark masses set to be $m_{c}^{\text {pole }}=1.3 \mathrm{GeV}$ in 
TABLE IV. Theory calculations for the high-precision data from the LHC which are newly included in the CT18(Z) global fit. The $K$ factors of ATL7WZ (Exp. ID 248) extracted from xFitter are calculated with DYNNLO and compared with FEWZ and MCFM in Appendix F.

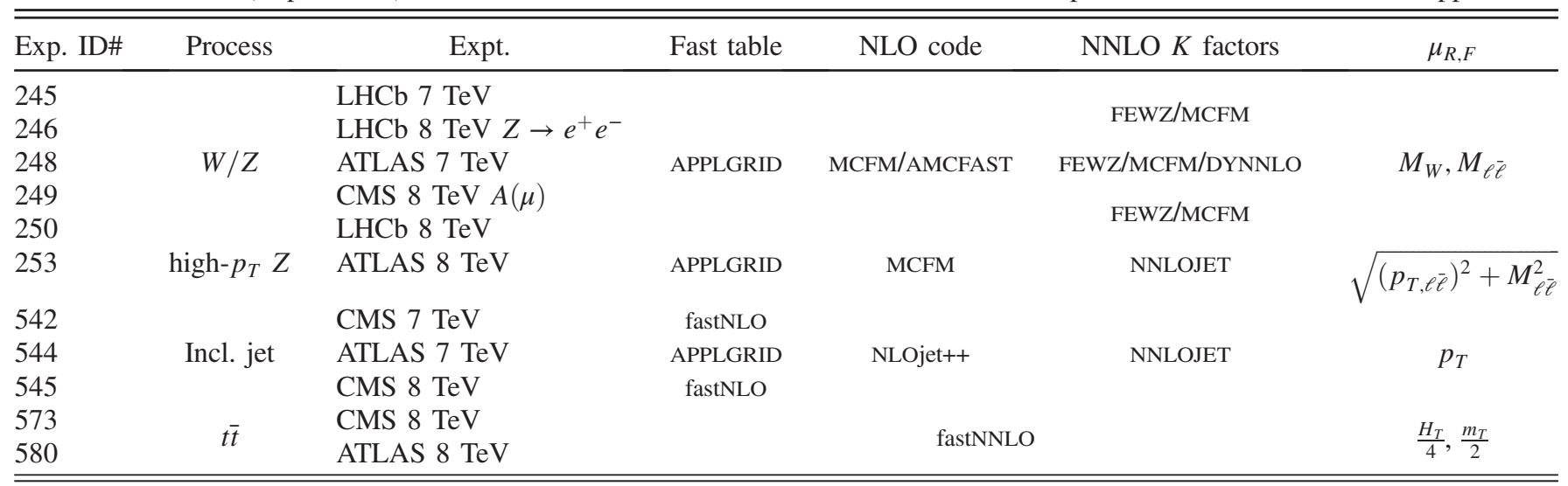

$\mathrm{CT} 18, \mathrm{~A}$, and $\mathrm{X}\left(m_{c}^{\mathrm{pole}}=1.4 \mathrm{GeV}\right.$ in $\left.\mathrm{CT} 18 \mathrm{Z}\right)$, and $m_{b}^{\text {pole }}=$ $4.75 \mathrm{GeV}$. The neutral-current DIS cross sections are evaluated at NNLO directly in the fitting code. For charged-current DIS cross sections, the NNLO cross sections from heavy quarks can be obtained by fast interpolations with pregenerated grids based on the calculation presented in Ref. [122]. The impact of the NNLO contribution on the description of the charged-current dimuon DIS data is further discussed in Sec. V B 4.

The computational complexity of NNLO matrix elements precludes their direct evaluation for each fit iteration, particularly given the expansive size of the datasets fitted in CT18. Instead, for the newly included high-precision data from the LHC, APPLGRID [16] and fastNLO [15] fast tables have been generated using programs such as MCFM [46], NLOJET++ [123] and AMCFAST [124], to allow fast evaluation of the matrix elements as the PDF parameters are varied. NNLO cross sections are then evaluated using NNLO/NLO point-by-point $K$-factors determined using the fast tables and NNLO programs such as NNLOJET [11,12,52,53,125], FEWZ [49-51], MCFM [46-48], and DYNNLO $[44,45]$. One exception is the top-quark data from ATLAS and CMS, for which fastNNLO tables have been provided by the authors for the NNLO cross sections $[126,127]$. The programs used for the calculation of the cross sections for each dataset are summarized in Table IV. We have explored the impact of the choices of scales and NNLO programs for some datasets, but the variation is not included in the PDF uncertainties for various reasons. More details can be found in the rest of this paper.

In the newest NNLO calculations for the high- $p_{T} Z$ and inclusive jet production available to the CT18 analysis, the NNLO corrections were not perfectly smooth among the experimental bins because of the statistical uncertainty introduced by MC integration. The resulting artificial fluctuations (of the magnitude of less than a fraction of percent of the central cross section values) have elevated the values of $\chi^{2}$ in these precise measurements. Through examination of the kinematic dependence of the NNLO/NLO $K$ factors, we identified all such cases and approximated the $K$ factors by smooth functions during the PDF fit. To account for the uncertainty introduced by the smoothing of the $K$ factors, we included uncorrelated $\mathrm{MC}$ errors equal to $0.5 \%$ of the central data values in the affected processes in Secs. III B 2 and III B 3. The MC errors lowered the $\chi^{2}$ values for these processes without changing the central PDF fits. The MC errors were estimated from the maximal deviations of the individual $K$-factor values from the respective smooth functions, with $0.5 \%$ being the conservative upper bound reached for a fraction of the fitted data points.

Error propagation must account for numerical theoretical errors of this kind. The non-negligible MC errors in some NNLO predictions were also noticed by other PDF fitting groups. The NNPDF group, for example, takes a similar approach in their analysis [3]. For the inclusive jet data, they use NLO calculations as the theoretical predictions, together with an additional correlated uncertainty estimated from the renormalization and factorization scale variations. For the high- $p_{T} Z$ boson data, an NNPDF-based analysis also adds an extra $1 \%$ uncorrelated uncertainty to account for the Monte Carlo fluctuations of the NNLO/NLO $K$-factor values [47].

For the legacy data on electroweak boson production, already included in the CT14 and CT14 ${ }_{\text {HERAII }}$, we inherit the original CTEQ calculations summarized in Table V. The NLO calculation is directly performed by the CT fitting code, while the point-by-point $K$ factors are calculated with VRAP [128,129], RESBOS [54,130], and FEWZ [49-51].

Even with the use of stored grids for fast evaluation of the matrix elements, significant improvements on speed are needed. The CT fitting code has been upgraded to a multithreaded version with a two-layer parallelization, through a rearrangement of the minimization algorithm and via a redistribution of the datasets. As a result, the speed of calculations increased by up to a factor of 10 . Details are provided in Appendix D. 
TABLE V. Theory calculations for the CT14 and CT14 HERAII's legacy data of electroweak vector boson production.

\begin{tabular}{|c|c|c|c|c|}
\hline $\begin{array}{l}\text { Exp. } \\
\text { ID\# }\end{array}$ & Experiment & $\begin{array}{l}\text { NLO } \\
\text { code }\end{array}$ & $\begin{array}{l}\text { NNLO } K \\
\text { factors }\end{array}$ & $\mu_{R, F}$ \\
\hline 201 & E605 DY & \multirow{4}{*}{ CTEQ } & \multirow{4}{*}{ FEWZ } & \multirow{3}{*}{$M_{\ell \bar{\ell}}$} \\
\hline 203 & E866 DY $\sigma_{p d} / \sigma_{p p}$ & & & \\
\hline 204 & E866 DY $\sigma_{p p}$ & & & \\
\hline 225 & CDF run-1 $A(e)$ & & & $M_{\ell \bar{\ell}}$ \\
\hline 227 & CDF run-2 $A(e)$ & \multirow{3}{*}{ CTEQ } & \multirow{3}{*}{ RESBOS } & \multirow{3}{*}{$M_{W}$} \\
\hline 234 & $\mathrm{D} \varnothing \operatorname{run}-2 A(\mu)$ & & & \\
\hline 281 & $\mathrm{D} \varnothing \operatorname{run}-2 A(e)$ & & & \\
\hline 260 & DØ run-2 $y_{Z}$ & \multirow{2}{*}{ CTEQ } & \multirow{2}{*}{ VRAP } & \multirow{2}{*}{$M_{\ell \bar{\ell}}$} \\
\hline 261 & CDF run-2 $y_{Z}$ & & & \\
\hline 266 & CMS $7 \mathrm{TeV} A(\mu)$ & \multirow{3}{*}{ CTEQ } & \multirow{3}{*}{ RESBOS } & \\
\hline 267 & CMS $7 \mathrm{TeV} A(e)$ & & & \\
\hline 268 & $\begin{array}{c}\text { ATLAS } 7 \mathrm{TeV} 2011 \\
W / Z\end{array}$ & & & $M_{W}, M_{\ell \bar{\ell}}$ \\
\hline
\end{tabular}

We will now describe the theoretical calculations for each new LHC process included in the CT18(Z) fits.

\section{LHC inclusive jet data}

LHC inclusive jet data are available with different jet radii. We have chosen the larger of the two nominal jet radii, 0.6 for ATLAS and 0.7 for CMS, to reduce dependence on resummation/showering and hadronization effects [131]. There is a non-negligible difference at low jet transverse momentum between theory predictions at NNLO using as the momentum-scale choice of either the inclusive jet $p_{T}$ or the leading jet $p_{T}\left(p_{T 1}\right)$ [13]. The nominal choice adopted by the CTEQ-TEA group is to use the inclusive jet $p_{T}$. We have observed that the fitted gluon PDF is not very sensitive to this choice even in the kinematic regions where the difference in NNLO predictions between these two scale choices is important.

Electroweak corrections from Ref. [132] were applied to jet cross sections and can be as large as 10\% for the highest transverse momentum bin in the central rapidity region, but decrease quickly with increasing rapidity and with decreasing jet transverse momentum. Furthermore, in accord with the previous subsubsection, the QCD NNLO/NLO $K$ factors were fitted with smooth curves, and a $0.5 \%$ theoretical error assessed with respect to the data has been added to each data value to take into account the fluctuations in integration of NNLO cross sections provided by NNLOJET.

\section{LHC electroweak gauge boson hadroproduction}

The Drell-Yan theory calculations at NNLO in the CT18 (Z) global analysis consist of the following:

(1) ATLAS $7 \mathrm{TeV} 4.6 \mathrm{fb}^{-1}$ measurements of $W^{ \pm}$and $Z / \gamma^{*}$ production cross sections in the $e$ and $\mu$ decay channels [39]: the theory predictions at NLO are obtained by using APPLGRID [16] fast tables generated with MCFM [46] and validated against AMCFAST [124] interfaced with MadGraph5_aMC@NLO [133]. The NNLO corrections are imported from the xFitter analysis published in Ref. [39]. These corrections are obtained using the DYNNLO- 1.5 code $[44,45]$, and checked against FEWZ-3.1.B2 [49-51] and MCFM8.0 [47] codes. Some discrepancy among these codes (up to $\sim 1 \%$ ) were found. However, these discrepancies do not induce significant differences in the calculated results like $\chi^{2}$. More details can be found in Appendix F.

(2) CMS $8 \mathrm{TeV} 18.8 \mathrm{fb}^{-1}$ measurements of muon charge asymmetry [83]: the theory predictions at NLO are from APPLGRID generated with MCFM, while for the NNLO corrections, we use $K$ factors calculated with FEWZ-3.1. These predictions have also been validated with MCFM-8.0.

(3) $\mathrm{LHCb} 7 \mathrm{TeV} W / Z$ cross sections, $W$ charge asymmetry measurements with $1 \mathrm{fb}^{-1}$ of integrated luminosity [81], and $\mathrm{LHCb} 8 \mathrm{TeV}$ measurements including both the electron [82] and muon [84] channels: the NLO theory calculation is obtained by using APPLGRID fast tables generated with MCFM. These have been validated against MadGraph5 aMC@NLO + AMCFAST. The NNLO corrections are calculated with FEWZ, and validated by MCFM.

(4) ATLAS [85,104] and CMS [105] measurements of transverse momentum of Drell-Yan lepton pairs at $7 \mathrm{TeV}$ and/or $8 \mathrm{TeV}$. The CT18(Z) fit includes only the ATLAS $8 \mathrm{TeV}$ absolute differential cross section measurements. The NLO theoretical calculation is performed with APPLGRID generated with MCFM. The NNLO corrections are provided by the NNLOJET group $[52,53]$. We have fitted the NNLO/NLO $K$ factors with smooth curves and include a $0.5 \% \mathrm{MC}$ error to account for the fluctuations in the NNLO calculations. In addition, we have imposed the kinematic cut $45<p_{T, \ell \bar{\ell}}<150 \mathrm{GeV}$ to ensure reliability of the fixed-order calculation. The low$p_{T, \ell \bar{\ell}}$ region is dropped due to the non-negligible contribution from QCD soft-gluon resummation, and the high $p_{T, \ell \bar{\ell}}$ region is dropped because the EW corrections there are expected to grow $[134,135]$.

\section{Top-quark pair production}

Theory predictions for top-quark pair production differential distributions at the LHC $8 \mathrm{TeV}$ are implemented at NNLO in QCD using fastNNLO tables $[17,18]$. In the CT18 global fit, the top-quark mass has been set to $m_{t}^{\text {pole }}=173.3 \mathrm{GeV}$. Motivated by [126], we chose the default central scale $\mu_{F, R} \equiv \mu=1 / 2 \sqrt{m_{t}^{2}+p_{T, t}^{2}}$ for the top-quark $p_{T}$ spectrum, while the rest of the distributions are 
obtained with $\mu=1 / 4\left(\sqrt{m_{t}^{2}+p_{T, t}^{2}}+\sqrt{m_{t}^{2}+p_{T, \bar{t}}^{2}}\right)$. The impact of the electroweak (EW) corrections on the theory predictions

for $t \bar{t}$ differential distributions has been studied in [136] where the difference between the additive and multiplicative approaches for combining QCD and EW corrections is also investigated. EW $K$ factors from an analytic fit for the $\mathrm{QCD} \times \mathrm{EW} / \mathrm{QCD}$ contributions are available [137]. The CT18 global analysis does not include EW corrections in $\bar{t} t$ production. Their impact on the fitted PDFs is expected to be small in the kinematic range of the differential distributions currently considered.

The impact of the EW corrections on the CT18 theory predictions at CMS $13 \mathrm{TeV}$ is illustrated in Sec. VI. In this case, the CT18 theory predictions include EW corrections evaluated using the multiplicative approach of [136], and the recommended value of $m_{t}^{\text {pole }}=172.5 \mathrm{GeV}$ has been used to compare theory and the CMS data (without fitting the data).

\section{Parametrization forms, systematic errors, and final PDF uncertainty}

\section{Nonperturbative parametrization forms}

An important source of the uncertainty in the CTEQ PDF analysis is associated with the choice of the parametric form for the fitted distributions at the lower boundary of QCD evolution, $f_{a}\left(x, Q=Q_{0}\right)$. There is limited guidance from theory as to the most appropriate PDF parametrizations, and it is favorable to guarantee a maximal level of parametric flexibility without overfitting experimental data [37]. In Appendix C, we present the explicit parametrization forms used in CT18. As usual, the PDFs at higher scales $Q>Q_{0}$ are computed using the Dokshitser-GribovLipatov-Altarelli-Parisi equations at NNLO, with splitting kernels available from Refs. [138,139]. ${ }^{4}$

\section{Treatment of experimental systematic errors}

The experimental systematic errors are commonly published in the form of percentage tables and belong to one of two types: additive or multiplicative. An additive error is the one whose absolute value is known, for example the uncertainty of the pileup energy, or the underlying event energy. Most errors, though, are multiplicative, meaning that the error is determined as a fraction of the experimental cross section for that bin. An example is the jet energy scale uncertainty. There are a number of options as to how to evaluate both types of systematic errors. This topic was explored in depth in previous CT papers $[1,32,38,42,91,142,143]$.

The most natural choice may seem to simply multiply the fractional uncertainty corresponding to a particular systematic error by the experimental cross section in that

\footnotetext{
${ }^{4}$ Independent recent computations of these kernels are available in $[140,141]$.
}

bin. However, due to fluctuations, this choice can result in a bias in favor of experimental data points with lower central values, the so-called D' Agostini bias [144,145]. Instead, for CT18, as for CT14 and CT10, we use what we have termed the "extended- $T$ " option, where the systematic error for each multiplicative term is determined by multiplying the fractional uncertainty times the theoretical prediction for that bin, a quantity which is not subject to the same fluctuations. The theory, and thus the multiplicative error, is recalculated for every iteration of the global PDF fitting. In the case of inclusive jet production, we observe that the additive treatment of experimental systematic errors produces the gluon PDF that is substantially softer at $x>0.1$, the pattern that was already observed in the CT10 NNLO analysis (cf. Figs. 18 and 19 in Section VI.D of [42]).

\section{The final PDF uncertainty}

To estimate the parametrization dependence, we repeated the CT fits multiple times using a large number (more than 250) of initial parametrization forms which have comparable numbers of fitting parameters. Some candidate fits are based on the functional forms like the ones shown in Appendix C, but with alternative choices for the orders of Bernstein polynomials, relations between the $x$ and $y$ variables, and relations between the Bernstein coefficients $a_{i}$. In many of these 250 fits, we increased the number of free parameters in Bernstein polynomials for some flavors up to six or seven, or we used a different form of the variable $y$ defined after Eq. (C1) and before Eq. (C7), or we did not require $a_{2}$ to be the same for $u_{v}$ and $d_{v}$, and similarly sometimes we relaxed the equality relations on $a_{1}$ for $\bar{u}, \bar{d}, \bar{s}$.

In addition, we repeated some fits by randomly changing the treatment of some experimental systematic errors from multiplicative to additive. Yet another class of candidate fits is obtained by choosing alternative QCD scales in sensitive experiments such as high- $p_{T} Z$ boson production, or alternative codes to compute the NNLO $K$ factors, cf. Appendix F. The final PDFs are obtained using a fixed parametrization form and systematic parameter settings, but the uncertainty is computed according to the two-tier convention adopted in Refs. [38,42] so as to cover the bulk of the solutions obtained with the alternative choices. The results of this study are illustrated in Fig. 6, showing a selection of central fits (green solid curves) for a range of alternative fitting forms and multiplicative/additive choices for systematic errors in the LHC and Tevatron jet production, superposed within the uncertainty band (at the $68 \%$ confidence level) for the published version of CT18.

As we increased the number of free PDF parameters, a mild improvement (up to several tens of units) in the global $\chi^{2}$ or individual $S_{E}$ values was typically found, so long as $\lesssim 30$ free parameters were fitted. With more than about 30 parameters, the fits tend to destabilize as expanded parametrizations attempt to describe statistical noise. The final PDFs are based on the parametrizations with a total of 29 free parameters. For each of the four fits, we provide twice 

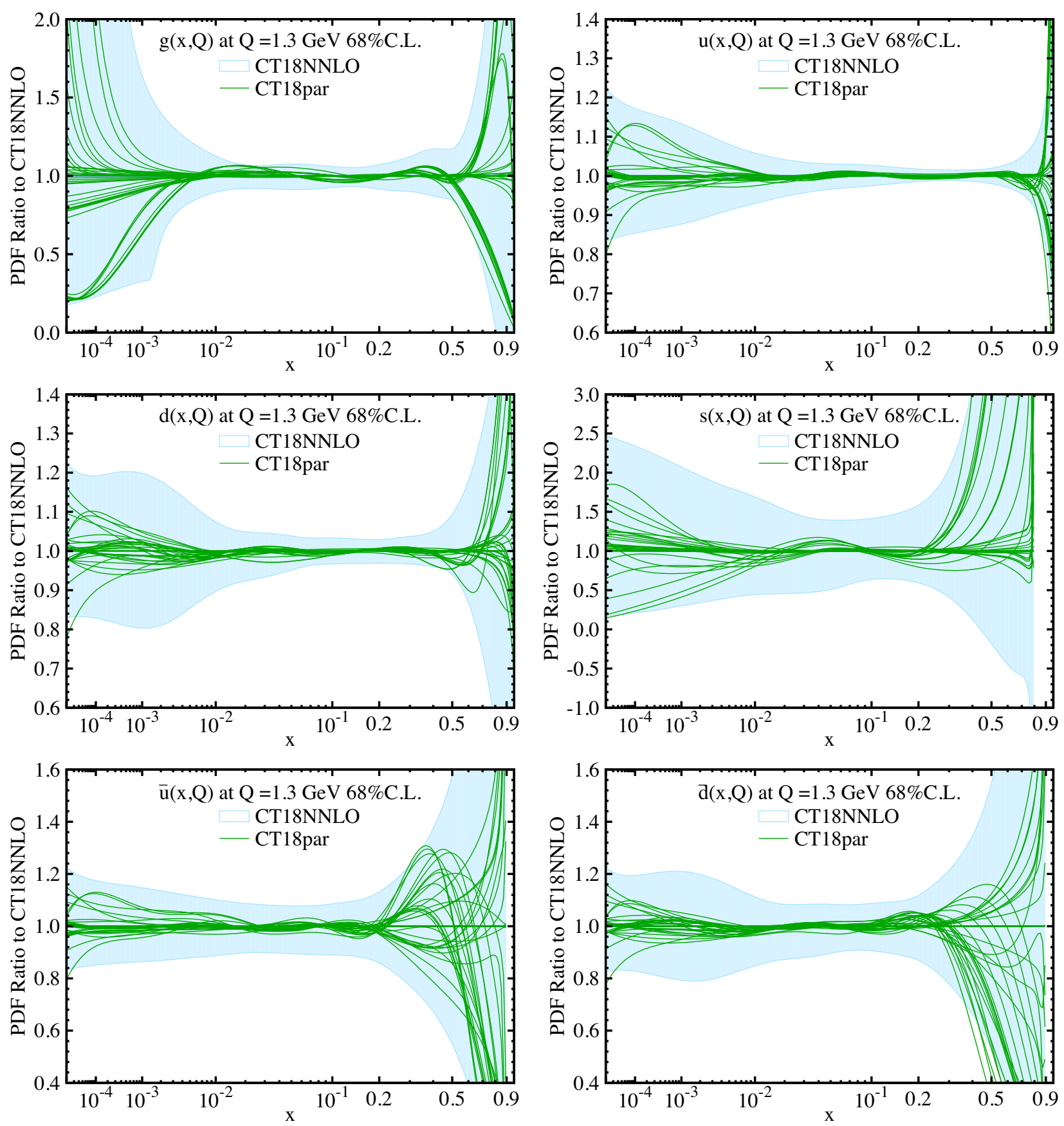

FIG. 6. To understand the parametrization dependence in the CT18 fit, we performed $\mathcal{O}(250)$ candidate PDF analyses using a wide range of alternative functional forms for $f_{a}\left(x, Q_{0}\right)$. The green curves in the panels above illustrate the spread of central fits achieved with the various candidate fits, evaluated as ratios with respect to the central CT18 fit.

as many Hessian error PDFs to evaluate the PDF uncertainties according to the CTEQ6 master formulas [23].

\section{THE CT18 OUTPUT: PDFS, QCD PARAMETERS, PARTON LUMINOSITIES, MOMENTS}

In this section, we review the behavior of CT18 PDFs and corresponding parton luminosities, Mellin moments, and parameters of the QCD Lagrangian. Given the large number of figures, for CT18Z fits, this section shows only the most critical comparisons. The rest of counterpart illustrations for CT18Z PDFs are presented in Appendixes A 3 a and A 4.

\section{A. Parton distributions as functions of $x$ and $Q$}

\section{PDFs for individual flavors}

Figure 2 shows an overview of the CT18 parton distribution functions, for $Q=2$ and $100 \mathrm{GeV}$. The function 

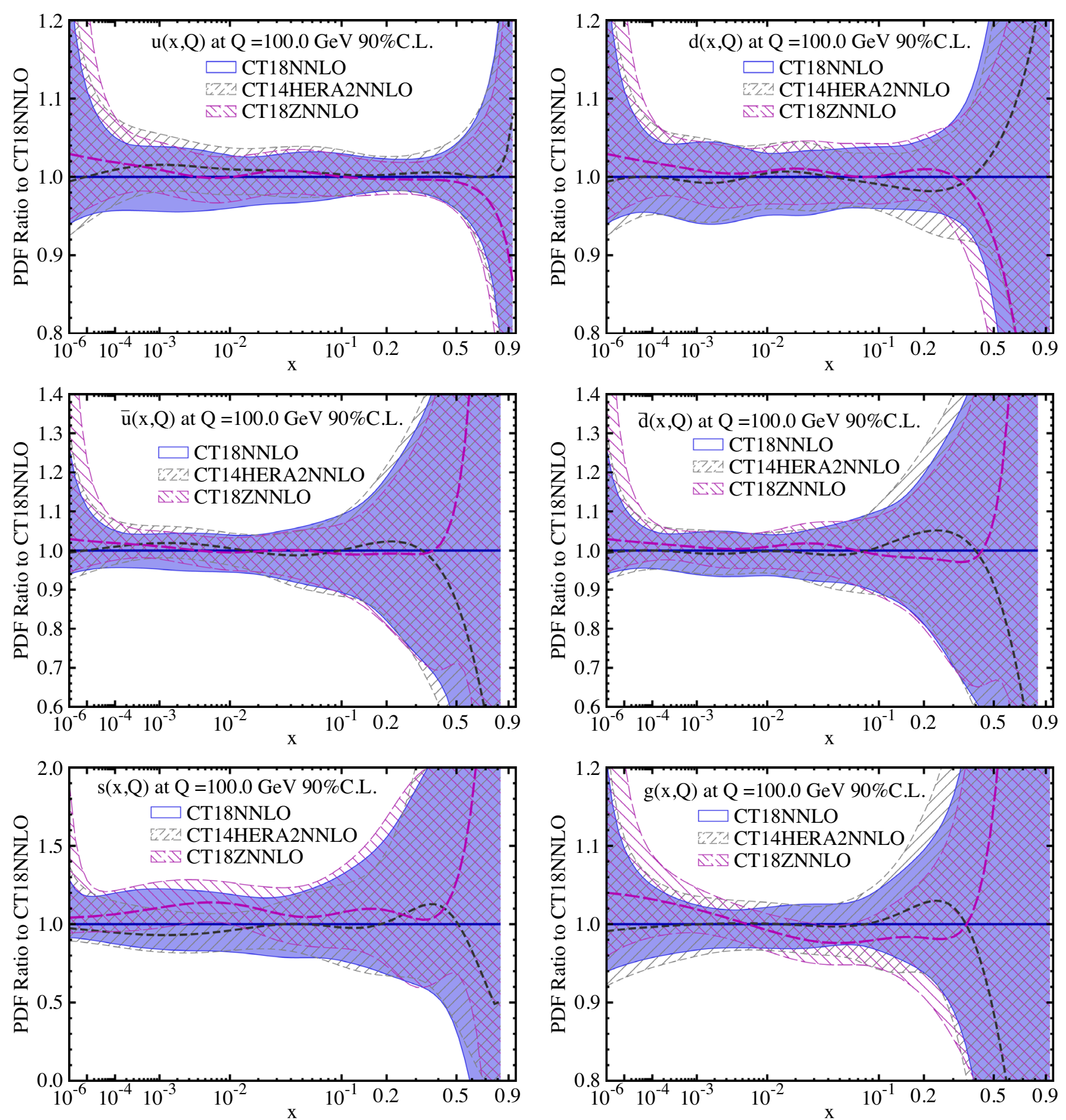

FIG. 7. A comparison of $90 \%$ C.L. PDF uncertainties from CT18 (violet solid), CT14 ${ }_{\text {HERAII }}$ (gray short-dashed line), and CT18Z (magenta long-dashed line) NNLO ensembles at $Q=100 \mathrm{GeV}$. The uncertainty bands are normalized to the central CT18 NNLO PDFs.

$x f(x, Q)$ is plotted versus $x$, for flavors $u, \bar{u}, d, \bar{d}, s=\bar{s}$, and $g$. We assume $s\left(x, Q_{0}\right)=\bar{s}\left(x, Q_{0}\right)$, since their difference is consistent with zero and has large uncertainty [146]. The plots show the central fit to the global data listed in Tables I and II, corresponding to the lowest total $\chi^{2}$ for our choice of PDF parametrizations. These are displayed with error bands representing the PDF uncertainty at the $90 \%$ confidence level (C.L.).
The relative changes from CT14 $4_{\text {HERAII }}$ NNLO to CT18 NNLO PDFs are best visualized by comparing their associated PDF uncertainties. Figure 7 compares the PDF error bands at $90 \%$ C.L. for the key flavors, with each band normalized to the corresponding best-fit CT18 NNLO PDF, represented by the solid violet line/bands. The long-dashed magenta and short-dashed gray curves/bands correspond to the CT18Z and CT14 $4_{\text {HERAII }}$ NNLO PDFs at $Q=100 \mathrm{GeV}$, 

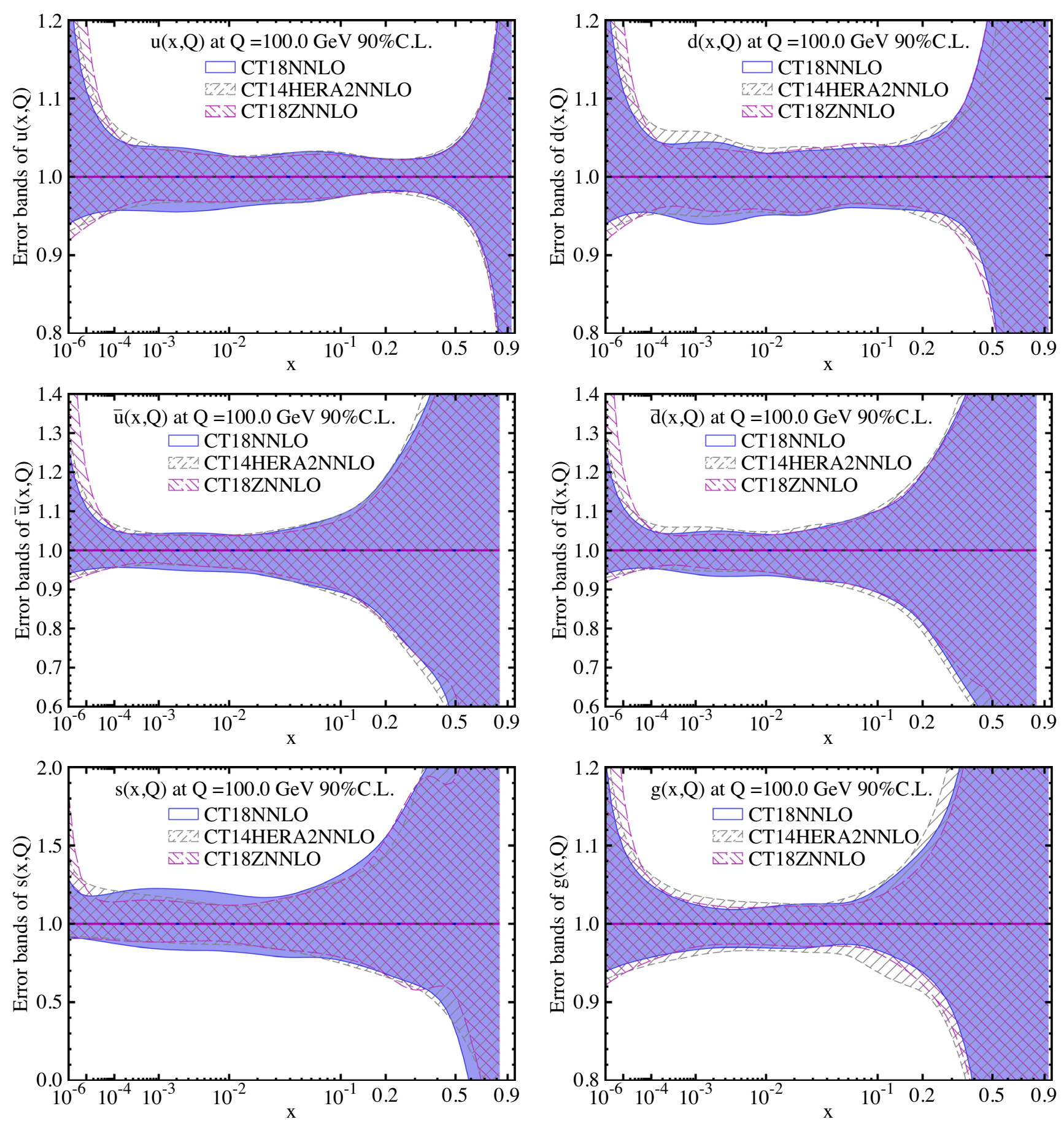

FIG. 8. Like Fig. 7, but with each error band normalized to its respective central PDF for the purpose of directly comparing the PDF uncertainties in CT18 NNLO with CT18Z and CT14 ${ }_{\text {HERAII }}$ NNLO.

respectively. Figure 8 shows the same error bands normalized to their respective central fits to facilitate comparison of their PDF uncertainties.

We make a number of observations for the NNLO PDFs. The CT18 $u$ PDF becomes slightly smaller, compared to CT14 $4_{\text {HERAII }}$, at almost all $x$ values, with the largest decrease at $x \sim 10^{-3}$. The $d$ PDF has increased at $x \sim 10^{-3}$ and $x \sim 0.2$, while it slightly decreased at $x \sim 0.01$. The $\bar{u}$ and $\bar{d}$ distributions are both smaller at $x \sim 0.3$ and larger at $x \sim 0.05$, though the decrease in $\bar{d}$ is larger. Furthermore, except for the $d$ PDF at $x \sim 0.2$, the error bands of $u, d, \bar{u}$ and $\bar{d}$ are about the same as CT14 HERAII. The central strangeness $(s)$ PDF has increased for $x<0.01$ and decreased for $0.2<x<0.5$, where the strange quark 

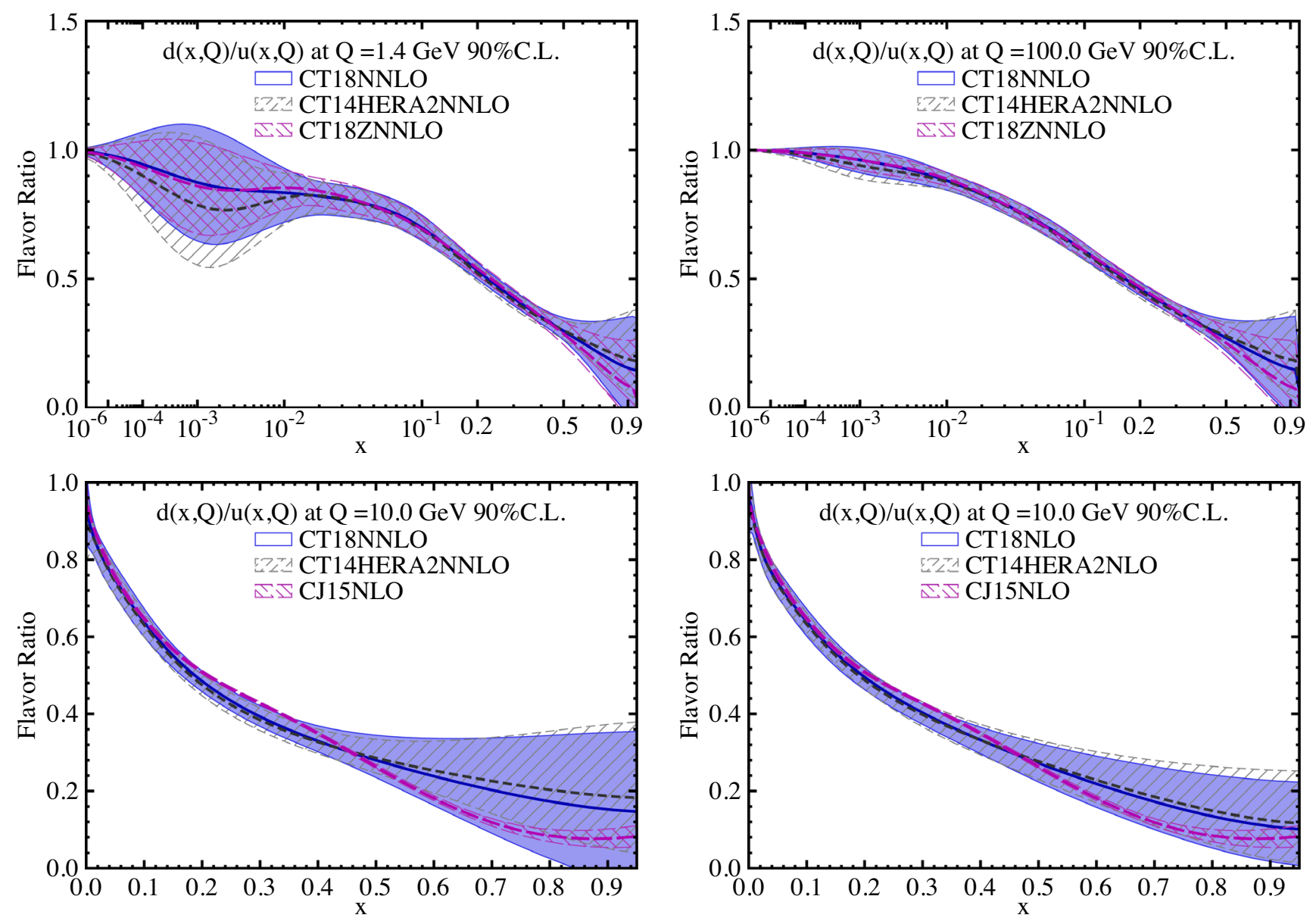

FIG. 9. Top: $90 \%$ C.L. uncertainties on the ratio $d(x, Q) / u(x, Q)$ for CT18, CT14 $4_{\text {HERAII }}$, and CT18Z NNLO ensembles at $Q=1.4$ and $100 \mathrm{GeV}$. Bottom: same, comparing CT18 and CT14 HERAII NNLO ratios (bottom-left) and respective NLO ratios (bottom-right) to the CJ15 NLO ensemble at $Q=10 \mathrm{GeV}$.

PDF is essentially unconstrained in CT18, just as in CT14 HERAII NNLO. Also, its uncertainty band is slightly larger than CT14 $4_{\text {HERAII }}$ for $x>10^{-4}$, as a consequence of the more flexible parametrization and the inclusion of the LHC data. We have checked that the most important datasets that drive the abovementioned changes in the quark and antiquark PDFs are the LHCb $W$ and $Z$ boson data, as listed in Table II with Exp. IDs $=250,245$, and 246 , with importance in that order. After including the LHCb $W$ and $Z$ boson data, the addition of CMS $8 \mathrm{TeV} W$ charge-asymmetry data $($ Exp. $I D=249)$ leads only to very mild changes in the CT18 PDFs. The central gluon PDF has decreased in CT18 at $x \approx 0.3$, with a smaller error band at $x \sim 0.1$ and below. The decrease of $g$ PDF for $0.1<x<0.4$ is caused by the inclusion of CMS and ATLAS jet data (with Exp. IDs $=545,543$, and 544, in that order) and ATLAS $8 \mathrm{TeV} Z$ boson transverse momentum $\left(p_{T}\right)$ data (Exp. ID = 253). With the LHC jet datasets already included, adding the ATLAS and CMS top-quark pair data (Exp. IDs $=580$ and 573) into the fit does not change the PDFs by a statistically significant amount.

\section{Ratios of PDFs}

Let us now review the ratios of various PDFs, starting with the ratio $d / u$ shown in Figs. 9 and 10. The changes in $d / u$ from CT18, as compared to CT14 $14_{\text {HERAII }}$, can be summarized as a reduction (increase) of the central ratio at $x>0.5$ $\left(x<10^{-2}\right)$ and a decreased uncertainty at $x<10^{-2}$. Beyond $x=0.5$, the error band of $d / u$ ratio grows, and the parametrization form adopted since CT14 NNLO [1] guarantees that $d / u$ approaches a constant value as $x \rightarrow 1$, as predicted by a wide array of theoretical models of nucleon structure. This is realized by equating the $(1-x)^{a_{2}}$ exponents of the $u_{v}$ and $d_{v}$ PDFs, i.e., $a_{2}^{u_{v}}=a_{2}^{d_{v}}$ (see Appendix C). This choice affects only the extrapolation to very high- $x$ values, $x \gtrsim 0.9$, beyond the range covered by the fitted data. At $x<0.9$, our parametrizations are flexible enough to cover the solutions and reproduce the uncertainty bands of the fits without this constraint. For example, the uncertainty band in Fig. 9 extends down to $d / u=0$ at $x=0.9$. Within the accessible $x$ reach, it also covers our candidate best fits with independent $a_{2}^{u_{v}}$ and $a_{2}^{d_{v}}$. Without this choice, the PDF ratio for an individual fitted PDF 

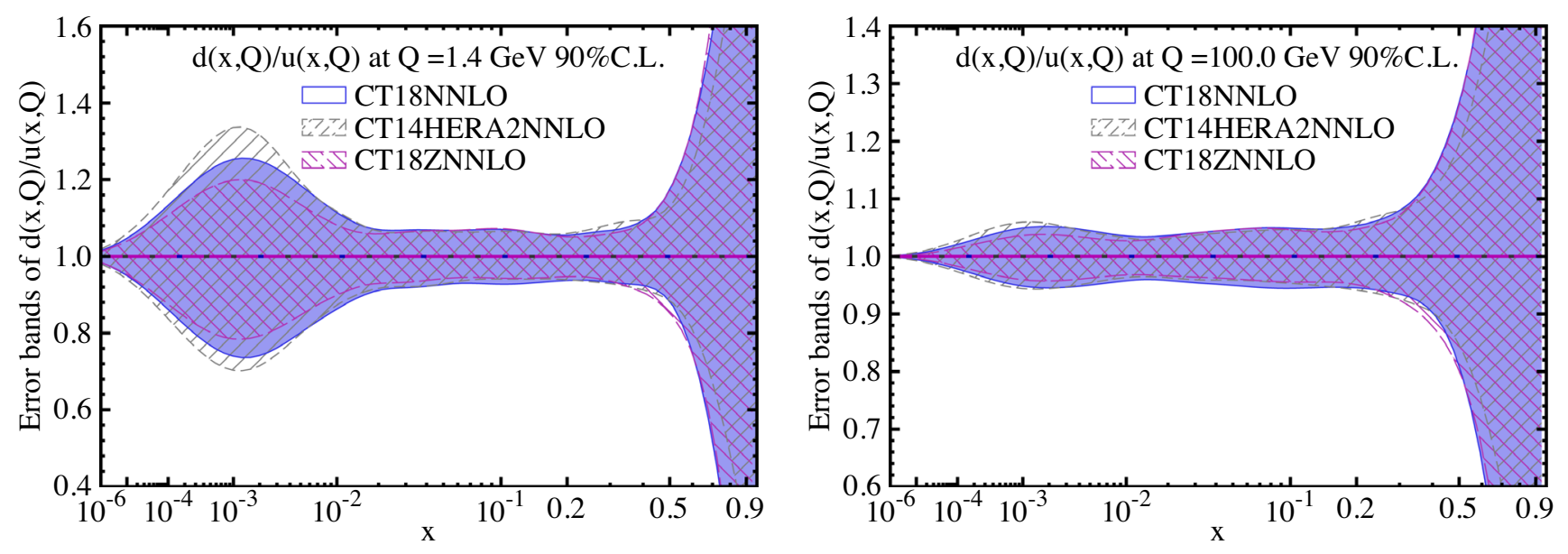

FIG. 10. Like the upper panels of Fig. 9 for $d / u$, but normalizing each fit to its respective central value to compare PDF uncertainties.

Hessian set would not have the parametric freedom to extrapolate to a finite constant at $x=1$. Instead, even minor differences in the fitted parameters, $a_{2}^{u_{v}} \neq a_{2}^{d_{v}}$, will cause it either to diverge or go to 0 at the highest- $x$ points, producing an infinite uncertainty on $d / u$ that is not compatible with the empirical electron-hadron data in that region or with common models of hadron structure. In a fit that does not constrain $a_{2}^{u_{v}}$ and $a_{2}^{d_{v}}$ to be the same, they may come up to be equal within the numerical precision of input parameters, however, in our extensive experience such coincidence hardly ever happens.

Similar logic applies to other PDF ratios, including the low- $x$ forms of the sea-quark distributions described below. As noted earlier, the parametrization form of $u, d, \bar{u}$, and $\bar{d}$ quarks in CT18 are the same as those in CT14 HERAII.

At such high $x$, the CTEQ-JLab analysis (CJ15) [5] has independently determined the ratio $d / u$ at NLO, by including the fixed-target DIS data at lower $W$ and higher $x$ that are excluded by the selection cut $W>3.5 \mathrm{GeV}$ in CT18, and by considering higher-twist and nuclear effects important in that kinematic region. Figure 9 shows that the central prediction of CT18 differs from CJ15 at $x>0.1$. The CT and CJ uncertainty bands are in mutual agreement, even though the error band of CJ15 is much smaller than CT18, a fact partly attributable to the $\Delta \chi^{2}=1$ criterion used in CJ15. Since the CJ15 PDFs are available only at NLO in $\alpha_{s}$, we compare the CJ15 NLO $d / u$ ratios to the respective CT18 NNLO (NLO) ratios in the bottom-left (bottom-right) frame of Fig. 9.

Turning now to the ratios of sea-quark PDFs in Fig. 11, we observe that the uncertainty on $\bar{d}(x, Q) / \bar{u}(x, Q)$ in the left inset has decreased at small $x$ in CT18. For $x>0.1$, the CT18 nonperturbative parametrization forms for $\bar{u}$ and $\bar{d}$ ensure that the ratio $\bar{d}\left(x, Q_{0}\right) / \bar{u}\left(x, Q_{0}\right)$ can approach a constant value, which turns out to be close to 1 in the central fit. The uncertainty on $\bar{d} / \bar{u}$ has also decreased, most notably for $x \gtrsim 10^{-3}$, primarily due to the inclusion of the
LHCb datasets (Exp. IDs $=250,245$ and 246), cf. the upper-left panel of Fig. 12 at $Q=1.4 \mathrm{GeV}$.

At high $Q$ values, such as in the right panels for $Q=$ $100 \mathrm{GeV}$ in Fig. 11, the ratios depend as much on the large$x$ gluon behavior at $Q_{0}$ as on the quark PDFs themselves. As a result, for CT18Z that has an enhanced gluon PDF and suppressed sea-quark PDFs at very large $x$ and $Q_{0}$, the uncertainties on the ratios $\bar{d} / \bar{u}$ and $R_{s}$ are reduced at $x \gtrsim 0.8$ and large $Q$, reflecting the flavor symmetry of $g \rightarrow q \bar{q}$ splittings that primarily drive the sea-quark PDFs in this $\{x, Q\}$ region.

The overall increase in the strangeness PDF at $x<0.03$ and decrease of $\bar{u}$ and $\bar{d}$ PDFs at $x<10^{-3}$, cf. Fig. 7, lead to a larger ratio of the strange-to-nonstrange sea-quark PDFs,

$$
R_{s}(x, Q) \equiv \frac{s(x, Q)+\bar{s}(x, Q)}{\bar{u}(x, Q)+\bar{d}(x, Q)}
$$

presented in Fig. 11. $R_{s}(x, Q)$ measures the $x$ and $Q$ dependence of the breaking of flavor-SU(3) symmetry, with older analyses typically fixing $R_{s}=0.5$. More recently, a number of previous CTEQ studies [146,147] examined contemporary constraints on $R_{s}$, particularly driven by the neutrino-induced SIDIS dimuon production measurements by the CCFR and NuTeV Collaborations, but also by precise inclusive HERA measurements. These works found significant evidence of an independent $x$ dependence for $s^{+}(x) \equiv s(x)+\bar{s}(x)$, distinct from $\bar{u}+\bar{d}$, but were unable to exclude a vanishing strangeness momentum fraction asymmetry, $\langle x\rangle_{s^{-}}=\int_{0}^{1} d x x[s-\bar{s}]\left(x, Q=m_{c}\right)=0$.

In the present work, we continue to assume $s^{-}(x, Q)=0$ and focus on $s^{+}(x, Q)$ and the related $R_{s}(x, Q)$, the quantities that both reflect the interplay of the older charged-current DIS data and new LHC measurements that are detailed later in Sec. V and Appendix A. Here let us mention that, at $x \ll 10^{-3}$, the $R_{s}$ ratio is determined entirely by the parametrization form and was found in 

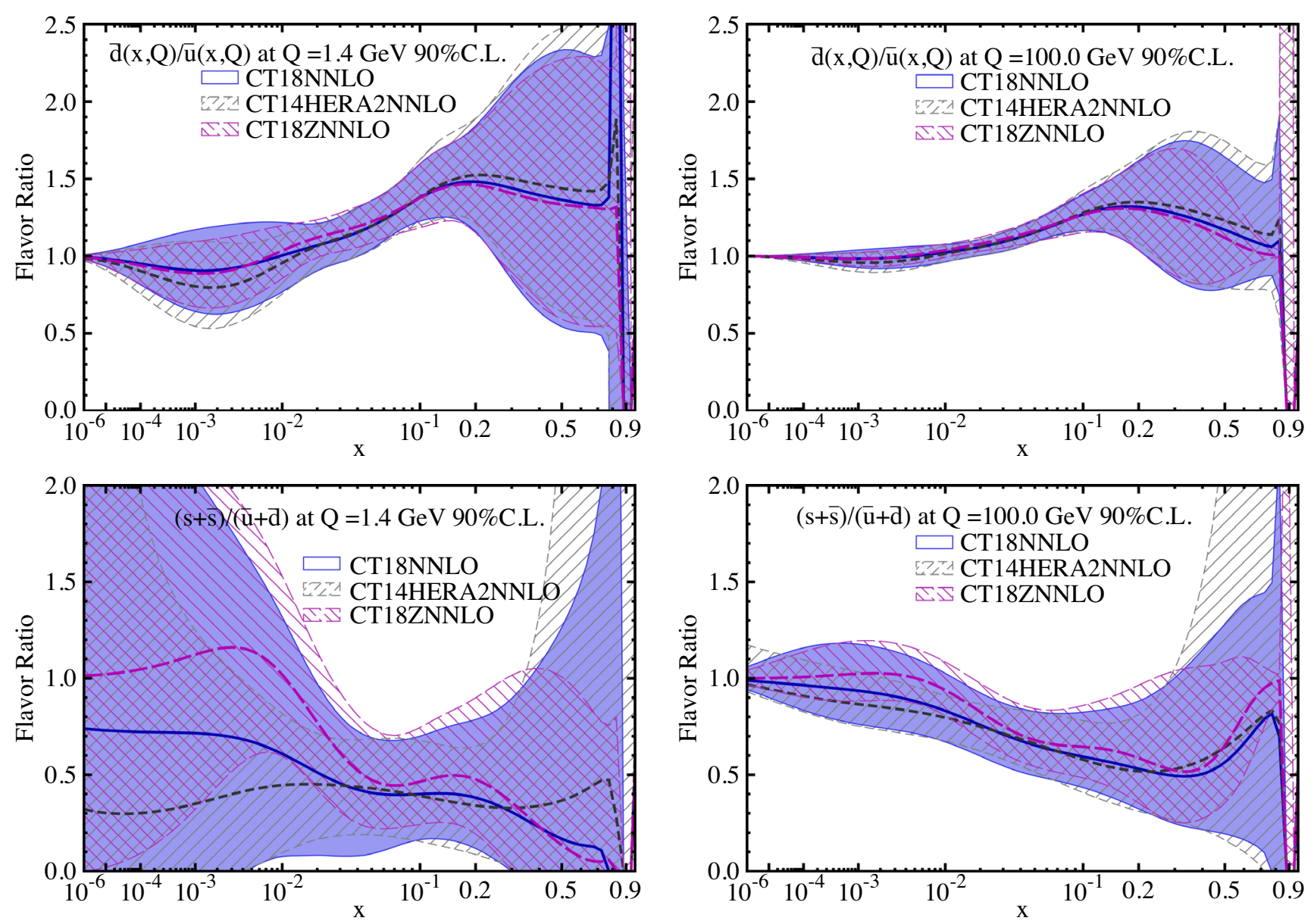

FIG. 11. A comparison of $90 \%$ C.L. uncertainties on the ratios $\bar{d}(x, Q) / \bar{u}(x, Q)$ and $(s(x, Q)+\bar{s}(x, Q)) /(\bar{u}(x, Q)+\bar{d}(x, Q))$, for CT18 (solid blue line), CT18Z (magenta long-dashed line), and CT14 $4_{\text {HERAII }}$ NNLO (gray short-dashed line) ensembles at $Q=1.4$ or $100 \mathrm{GeV}$.

CT10 to be consistent with the exact SU(3) symmetry of PDF flavors, $R_{s}(x, Q) \rightarrow 1$ at $x \rightarrow 0$, albeit with a large uncertainty. The $\mathrm{SU}(3)$-symmetric asymptotic solution at $x \rightarrow 0$ was not enforced in CT14 or CT14 HERAII, so that their $R_{s}$ ratio was around 0.3 to 0.5 at $x \approx 10^{-5}$ and $Q=1.4 \mathrm{GeV}$. In CT18, we have assumed a different $s$-PDF nonperturbative parametrization form (with one more parameter added), but the one that still ensures a stable behavior of $R_{s}$ for $x \rightarrow 0$, so that $R_{s}(x \rightarrow 0)$ is about 0.7 and 1, respectively, in CT18 and CT18Z fits.

\section{Changes in the $x$ dependence of PDFs, summary}

We may summarize the pulls of specific processes on the central CT18 fit as follows.

(1) The most noticeable overall impact of the LHC inclusive jet production on the central gluon PDF $g(x, Q)$ is to mildly reduce it at $x>0.2$ within the original PDF uncertainty band. The pulls from the jet datasets change little after the decorrelation of some systematic errors, cf. Sec. II B 4, and when the $0.5 \%$ MC uncertainty on theory values is added. The pulls from various jet datasets on $g(x, Q)$ neither follow a uniform trend across the whole $x$ range nor are consistent among various measurements, as is demonstrated, e.g., by the $L_{2}$ sensitivity in Fig. 26 and LM scans in Sec. VA.

(2) The LHCb data, combined over all processes, have some impact on the $u, d$ and $s$ quarks, and pull the $s(x, Q)$ up at small $x$.

(3) The ATLAS $8 \mathrm{TeV} Z p_{T}$ data (Exp. ID $=253$ ), for the nominal QCD scales assumed in the CT18 NNLO fits, weakly pull the gluon PDF at $x>$ 0.05 downward, in the direction similar to the average pull of the LHC inclusive jet data. The relative magnitude of the pull from these data, as compared to those from the jet experiments, can be estimated from the $L_{2}$ sensitivity plot for $g(x, Q)$ in Fig. 26.

(4) The ATLAS TeV data on $W$ and $Z$ rapidity distributions $($ Exp. ID $=248)$, included only in CT18A and $\mathrm{Z}$, have the largest influence on the PDFs, as discussed in Appendix A. The directions of their pulls are similar to $\mathrm{LHCb}$. 

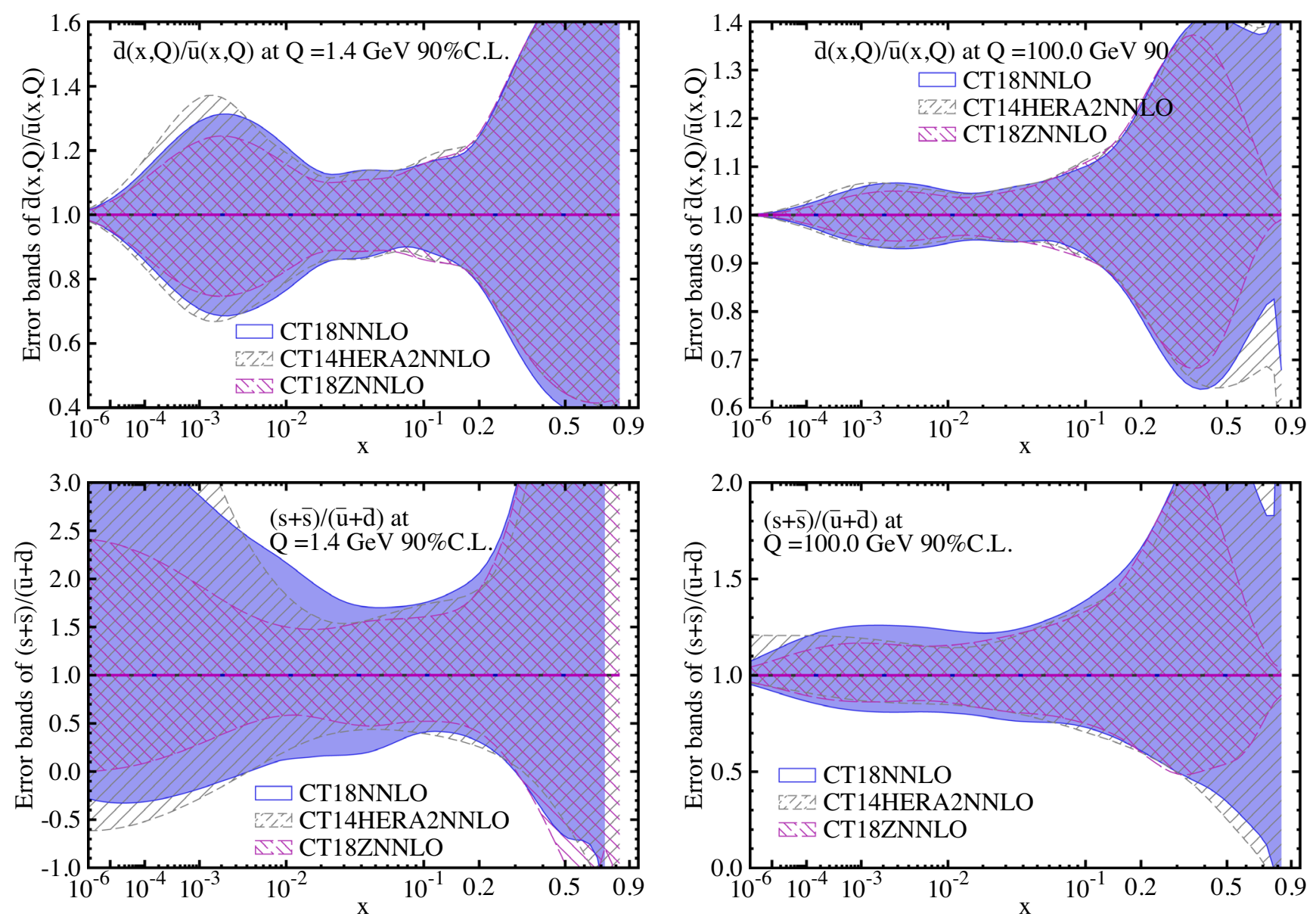

FIG. 12. A comparison of $90 \%$ C.L. uncertainties on the ratios $\bar{d}(x, Q) / \bar{u}(x, Q)$ and $(s(x, Q)+\bar{s}(x, Q)) /(\bar{u}(x, Q)+\bar{d}(x, Q))$, for CT18 (solid blue line), CT18Z (magenta long-dashed line), and CT14 $4_{\text {HERAII }}$ NNLO (gray short-dashed line) ensembles at $Q=1.4$ or $100 \mathrm{GeV}$, relative to their own central fit.

(5) The LHC data on $t \bar{t}$ double differential cross sections also appears to favor a softer gluon at large $x$, but the pull is not statistically significant, i.e., much weaker than that of the inclusive jet data with its much larger number of data points.

These constraints are further explored in depth in Sec. VA using a combination of statistical techniques.

\section{B. The global fits for $\alpha_{s}$ and $m_{c}$}

\section{Determination of the QCD coupling}

Following the long-established practice [29], in the canonical PDF sets such as CT18, the value of $\alpha_{s}\left(M_{Z}\right)$ is set to the world average of $\alpha_{s}\left(M_{Z}\right)=0.118$ [28]; alternate PDFs are produced for a range of fixed $\alpha_{s}\left(M_{Z}\right)$ above and below that central value (i.e., an " $\alpha_{s}$ series") to evaluate the combined PDF $+\alpha_{s}$ uncertainty. In Ref. [29], we show how to evaluate the combined PDF $+\alpha_{s}$ uncertainty in the global fit. As shown, variations in $\alpha_{s}$ generally induce compensating adjustments in the preferred PDF parameters (correlation) to preserve agreement with those experimental datasets that simultaneously constrain $\alpha_{s}$ and the PDFs. At the same time, it is possible to define an " $\alpha_{s}$ uncertainty" that quantifies all correlation effects. As the global QCD dataset grows in size, more experiments introduce sensitivity to $\alpha_{s}\left(M_{Z}\right)$ either through radiative contributions to hard cross sections or through scaling violations, especially over a broad range of physical scales, $Q$.

Perhaps the best way to examine the sensitivity of each experiment, and of the global ensemble of experiments, is to examine the variations of their $\chi^{2}$ as the value of $\alpha_{s}\left(M_{Z}\right)$ is varied. Such scans over $\alpha_{s}\left(M_{Z}\right)$ for CT18 NNLO and CT18 NLO are shown in Figs. 13 and 14, respectively. In all figures illustrating the scans in this and the next section, we plot a series of curves for

$$
\Delta \chi_{E}^{2}(a) \equiv \chi_{E}^{2}(a)-\chi_{E}^{2}\left(a_{0}\right)
$$

as a function of some parameter $a$. The variation $\Delta \chi_{E}^{2}(a)$ is the difference between the $\chi^{2}$ values for experiment $E$ at the fixed value of $a$ shown on the horizontal axis [with $\chi_{E}^{2}(a)$ 

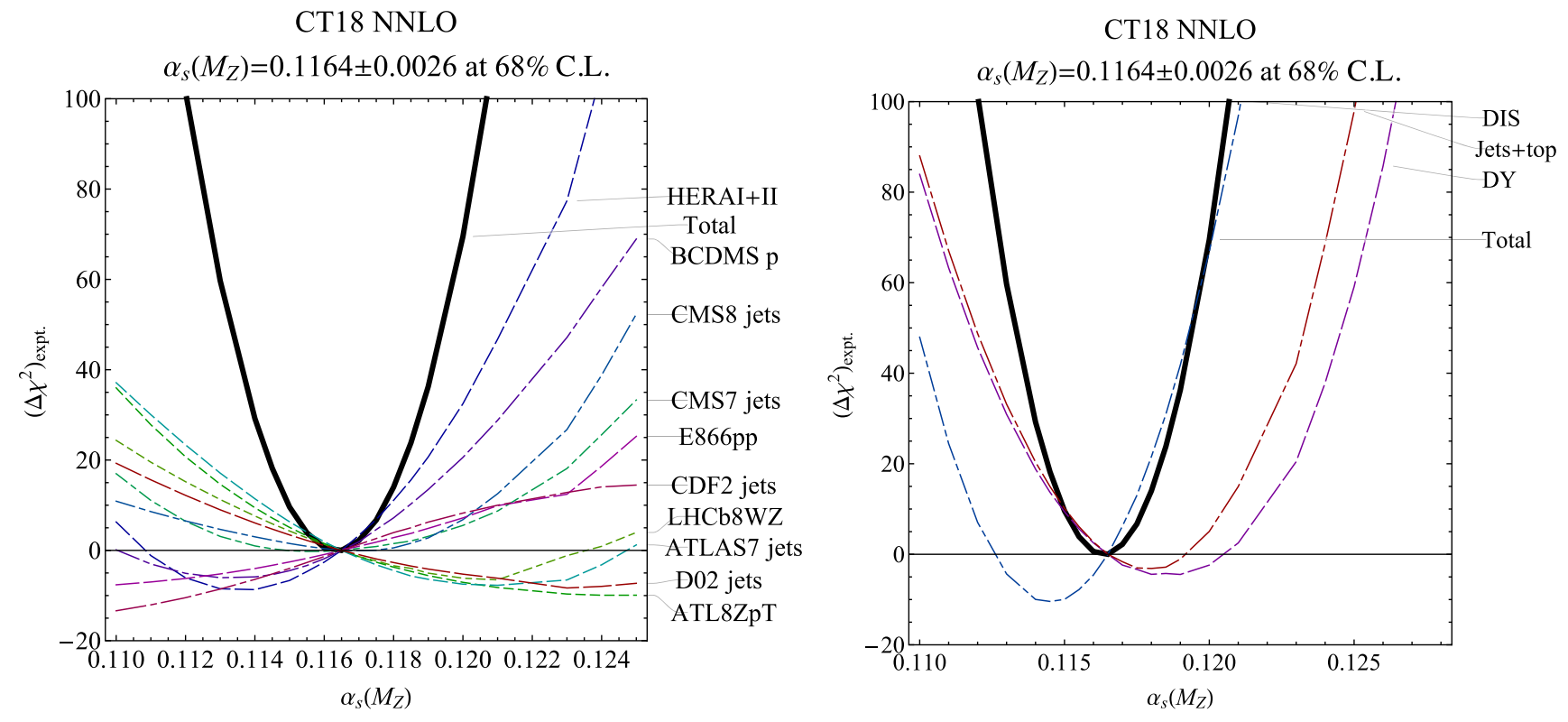

FIG. 13. The scan of the strong coupling constant at the scale of $M_{Z}$ for CT18 at NNLO. Left: changes of $\chi^{2}$ of all the datasets together (heavy black line) and of several individual experiments with especially strong pull on $\alpha_{s}\left(M_{Z}\right)$. Right: values for the change in $\chi^{2}$ for all experiments fitted in CT18, but separately collected into combined DIS, DY, and top/jets datasets. The growth in $\Delta \chi^{2}$ for the "jets+top" curve in the right panel is mainly driven by the constraints from jet production. While $t \bar{t}$ production has important sensitivity to $\alpha_{s}$, the comparatively small number of top data points leads to a more intermediate impact in the full fit.

marginalized with respect to the rest of free parameters], and when $a$ is determined at the global $\chi^{2}$ minimum for the full CT18 dataset, where $a=a_{0}$. The $\Delta \chi^{2}$ curves are shown for all experiments (indicated as "total" or " $\chi_{\text {tot }}^{2}$ ") and for the top few experiments with the largest variations $\Delta \chi_{E}^{2}$ in the shown range of $a$. Thus, by definition $\Delta \chi_{\text {tot }}^{2}\left(a_{0}\right)=0$.

We note that we have varied $\alpha_{s}$ in the present scan in all exact radiative contributions, but kept $\alpha_{s}$ fixed in the tabulated $K$ factors. This approximation greatly simplifies the computations, and we have verified that it changes $\chi^{2}$ by only a small fraction of the higher-order uncertainty within the fitted $\alpha_{s}$ range.
From Fig. 13, we see that the various datasets have different sensitivities to both the central value of $\alpha_{s}\left(M_{Z}\right)$ and its uncertainty. According to the scans, the greatest sensitivity to $\alpha_{s}\left(M_{Z}\right)$ is provided by the HERA I + II dataset, followed by the BCDMS proton data. Relatively to the full CT18 dataset, both experiments prefer a lower value of $\alpha_{s}\left(M_{Z}\right)$, on the order of $0.114-0.116$, but with wider uncertainties. The dependence of those two DIS datasets on $\alpha_{s}\left(M_{Z}\right)$ is primarily through the effect of scaling violation, but the sheer number of data points, and the experimental and theoretical precision, lead to their large sensitivities.

The LHC inclusive jet production, especially the CMS 7 and $8 \mathrm{TeV}$ data, generally prefer a large value of $\alpha_{s}\left(M_{Z}\right)$, as

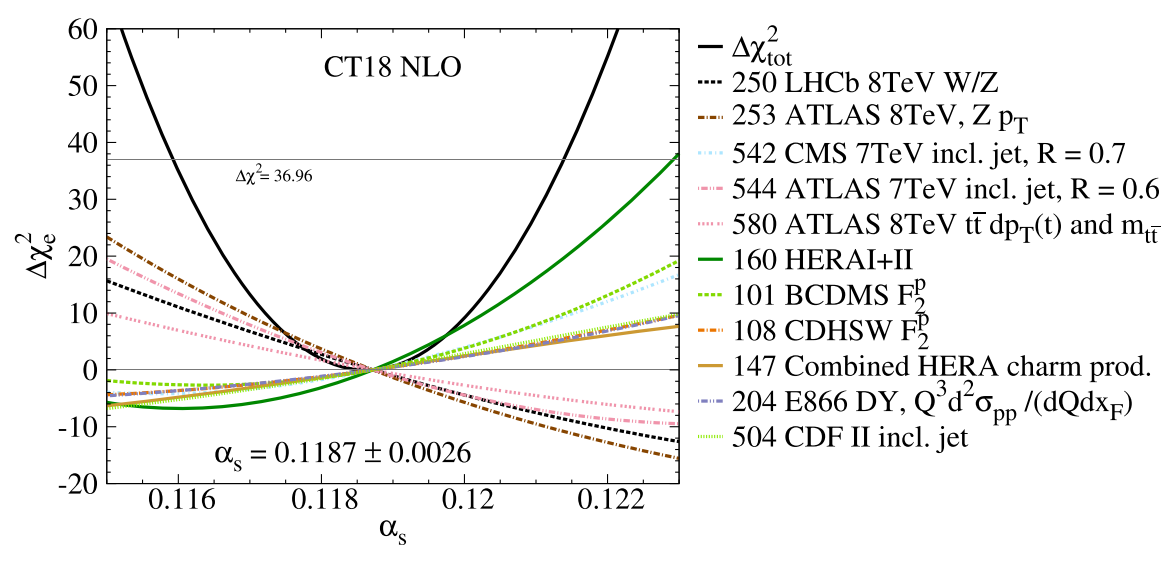

FIG. 14. Like Fig. 13, but now showing the scan of $\alpha_{s}\left(M_{Z}\right)$ at NLO precision in $\alpha_{s}$. 
does the ATLAS $8 \mathrm{TeV} \mathrm{Z} p_{T}$ data. The full CT18 dataset prefers a value of $\alpha_{s}\left(M_{Z}, \mathrm{NNLO}\right)=0.1164 \pm 0.0026$, at $68 \%$ C.L., defined using the "global tolerance" prescription to correspond to a $\Delta \chi^{2}=37$ interval (the corresponding $90 \%$ interval is defined by $\left.\Delta \chi^{2}=100\right)$. The extracted value of $\alpha_{s}\left(M_{Z}\right)$ obtained with $\mathrm{CT} 18 \mathrm{Z}$ is very similar, $0.1169 \pm 0.0026$, cf. Fig. 70 . These values are to be compared with $\alpha_{s}\left(M_{Z}\right)=0.1150_{-0.0024}^{+0.0036}$ as obtained by CT14 with a smaller HERA + LHC dataset.

The $\Delta \chi^{2}$ distribution for the full dataset is very parabolic, less so for the individual datasets. The $\Delta \chi^{2}$ curves for collections of datasets, for example, all DIS data, all DY data, and all jets and top data, also appear parabolic, as expected from the central limit theorem. From the right panel of Fig. 13, it is clear that the totality of DIS data prefer a smaller value of $\alpha_{s}\left(M_{Z}\right)$ than the DY pair, jet, and top-quark production. The exact size of the $\alpha_{s}$ uncertainty thus is not well determined and depends on the convention, as the pulls from various (types of) experiments are not consistent at the level of few tens of units of $\chi^{2}$.

The scan exercise can also be carried out at NLO in $\alpha_{s}$, as we show in Fig. 14. In fact, any difference between the NLO and NNLO results can serve as a partial estimate of the theoretical uncertainty of its determination. Although the uncertainty is similar to that obtained at NNLO, the central value is slightly higher: $\alpha_{s}\left(M_{Z}, \mathrm{NLO}\right)=0.1187 \pm$ 0.0027 . We note that the qualitative interplay of the experiments with leading sensitivity to $\alpha_{s}\left(M_{Z}\right)$ is much the same at NLO as found at NNLO, with the combined HERA (Exp. $\mathrm{ID}=160)$ and BCDMS $F_{2}^{p}$ data $($ Exp. ID = 101) again preferring lower values, while the ATLAS $7 \mathrm{TeV}$ jet data $($ Exp. ID $=544)$ and $8 \mathrm{TeV} Z p_{T}$ data $($ Exp. ID $=253)$ pulling in the opposing direction, but more strongly at NLO than at NNLO. The preference of a higher $\alpha_{s}$ value at NLO by an amount of about 0.002 is consistent with findings of other PDF groups $[4,30,148,149]$.

To summarize, we find that the CT18 dataset prefers a larger value of $\alpha_{s}\left(M_{Z}\right)$ and a marginally smaller nominal uncertainty than in CT14.

\section{Constraining the charm pole mass}

Similar investigations can be carried out for other inputs of the perturbative theory, such as the pole mass of the charm quark, $m_{c}$. A conclusive study on the charm mass dependence is beyond the scope of this article: the experimental preferences for $m_{c}$ may be affected by the initial scale $Q_{0}$, auxiliary settings in the heavy-quark scheme, and possibility of the nonperturbative charm $[56,121,150]$. In the candidate fits we made, we observe that the traditional choice $m_{c}^{\text {pole }}=1.3 \mathrm{GeV}$ remains compatible with the CT18(Z) global data, however, the most recent HERA inclusive DIS and LHC vector boson production experiments in totality may mildly prefer the pole mass of $1.4 \mathrm{GeV}$ or higher.

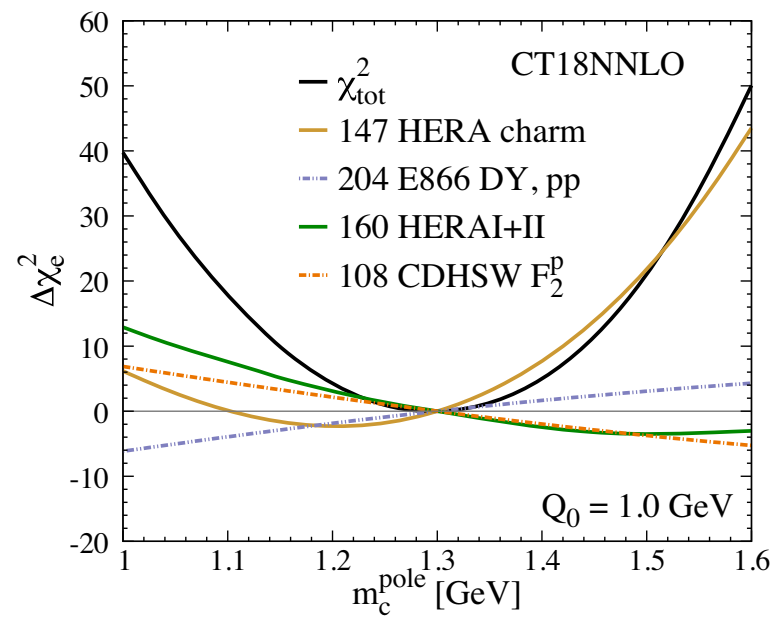

FIG. 15. A $\chi^{2}$ scan over values of the charm pole mass, $m_{c}$, at NNLO, using the CT18 dataset. The settings of the fit are described in the text. The CT18Z counterpart to this $m_{c}$ scan is presented in Fig. 71 in Appendix A.

An illustration of the observed trends can be viewed in Fig. 15, where we show the $\chi^{2}$ variation for the total dataset and for the leading experiments in a NNLO fit to the CT18 dataset at different pole $m_{c}$. To separate the $m_{c}$ dependence from $Q_{0}$ dependence, we set $Q_{0}=1 \mathrm{GeV}$ and use a more flexible (sign-indefinite at small $x$ ) gluon parametrization that better accommodates the full range of solutions at such low $Q$. The choice $Q_{0}=1 \mathrm{GeV}$, also used in the CT10 study [121] of $m_{c}$ dependence, allows us to widen the examined range of $m_{c}$, while the extra flexibility of the gluon at $Q=1 \mathrm{GeV}$ is needed to accommodate the full range of the CT18 PDF uncertainty at $Q>1.3 \mathrm{GeV}$. As can be seen based on the minimum of the heavy black curve, the scan prefers a value of $m_{c}=1.3 \mathrm{GeV}$, with this mass being somewhat larger than the preference of the combined charm production data from HERA (Exp. $\mathrm{ID}=147$ ) alone, which would otherwise suggest $m_{c} \gtrsim 1.2 \mathrm{GeV}$. The combined HERA run-I and run-II inclusive data, on the other hand, essentially provide a lower bound to $m_{c}$, and prefer a larger magnitude, $m_{c}>1.45 \mathrm{GeV}$. However, these preferences are quite weak, yielding an overall change by ten units of $\chi^{2}$ over a large range of $m_{c}$. The individual sensitivities of the other experiments presented in Fig. 15 are even weaker. It should be pointed out that the inclusion of the ATLAS $7 \mathrm{TeV} W / Z$ data (Exp. ID = 248) and other changes associated with CT18Z lead to a reconfiguration of the picture shown in Fig. 15 and to an increase in the best-fit value of $m_{c}$, as we show in Fig. 71 and discuss in Appendix A. In the same spirit, the patterns of the pulls change somewhat if we set $Q_{0}=m_{c}$ (another acceptable choice).

\section{Parton luminosities at the LHC}

In Fig. 16, we show the parton luminosities at the LHC $14 \mathrm{TeV}$ computed with the CT18 and CT18Z NNLO PDFs, 

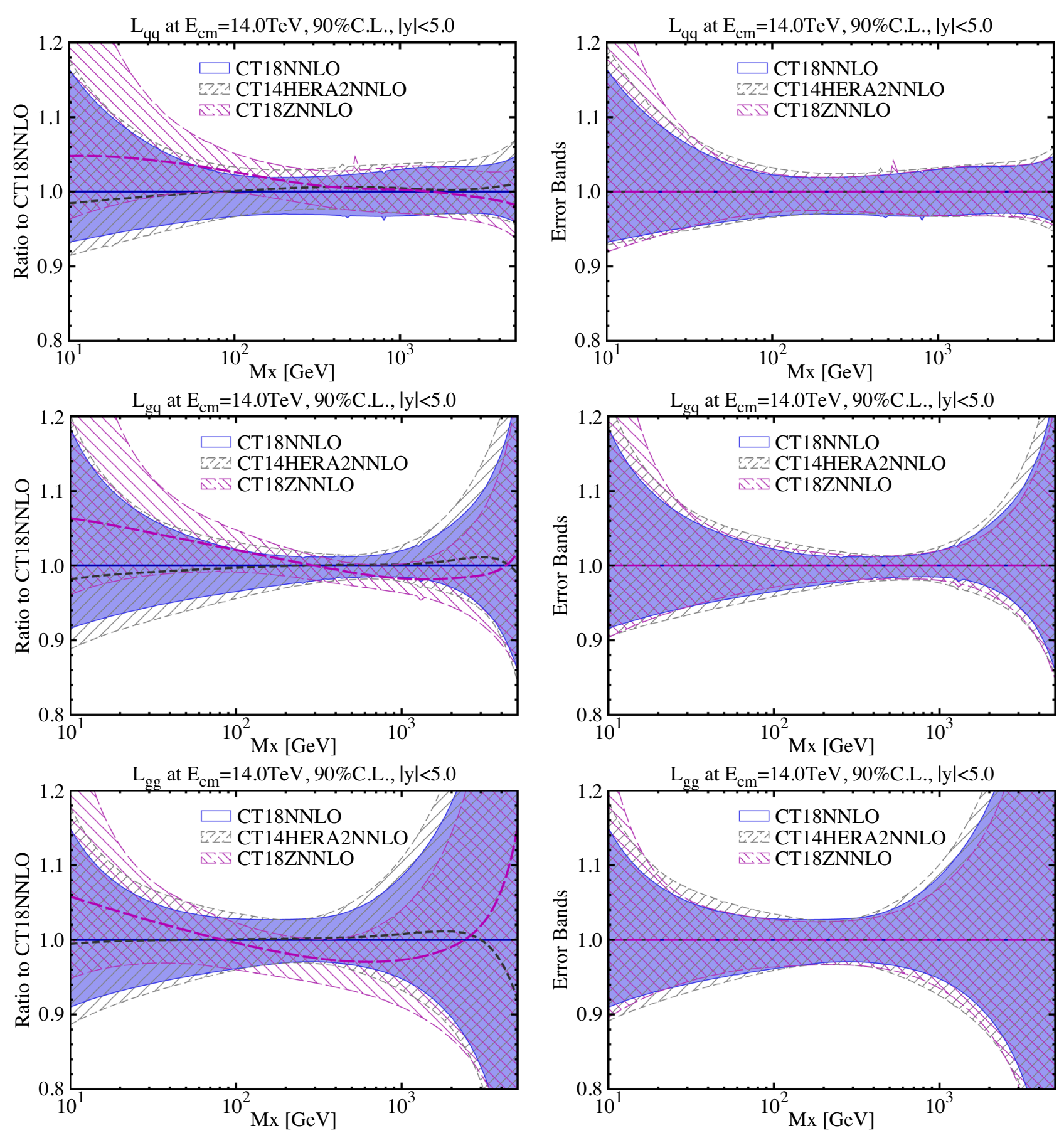

FIG. 16. Parton luminosities for processes at the LHC at $\sqrt{s}=14 \mathrm{TeV}$, in the central rapidity region $|y|<5: L_{q q}$ (upper panels), $L_{g q}$ (center panels), and $L_{g g}$ (lower panels); evaluated using CT18 (solid violet line), CT18Z (short-dashed gray line), and CT14 ${ }_{\text {HERAII }}($ longdashed magenta line) NNLO PDFs. The left panels give the luminosity ratios normalized to CT18, whereas the right panels show the error bands for each luminosity, normalized for each PDF ensemble to its own central prediction.

contrasting them with the previous CT14 $14_{\text {HERAII }}$ release. To compare the luminosities only within the physically accessible regions, we compute the integrals of the luminosity with a restriction on the absolute rapidity of the final state to be within five units, cf. Eq. (28) in [151]. In the comparisons for each flavor combination, we again show results normalized either to a common reference (either CT18
NNLO or NLO) in the left-hand plots or to their respective central predictions.

As in the case of individual PDFs, the CT18 central results for the parton luminosities remain very close to CT14 $4_{\text {HERAII }}$. On the other hand, the PDF uncertainties of the luminosities for the individual PDF ensembles are somewhat reduced, especially for those luminosities 

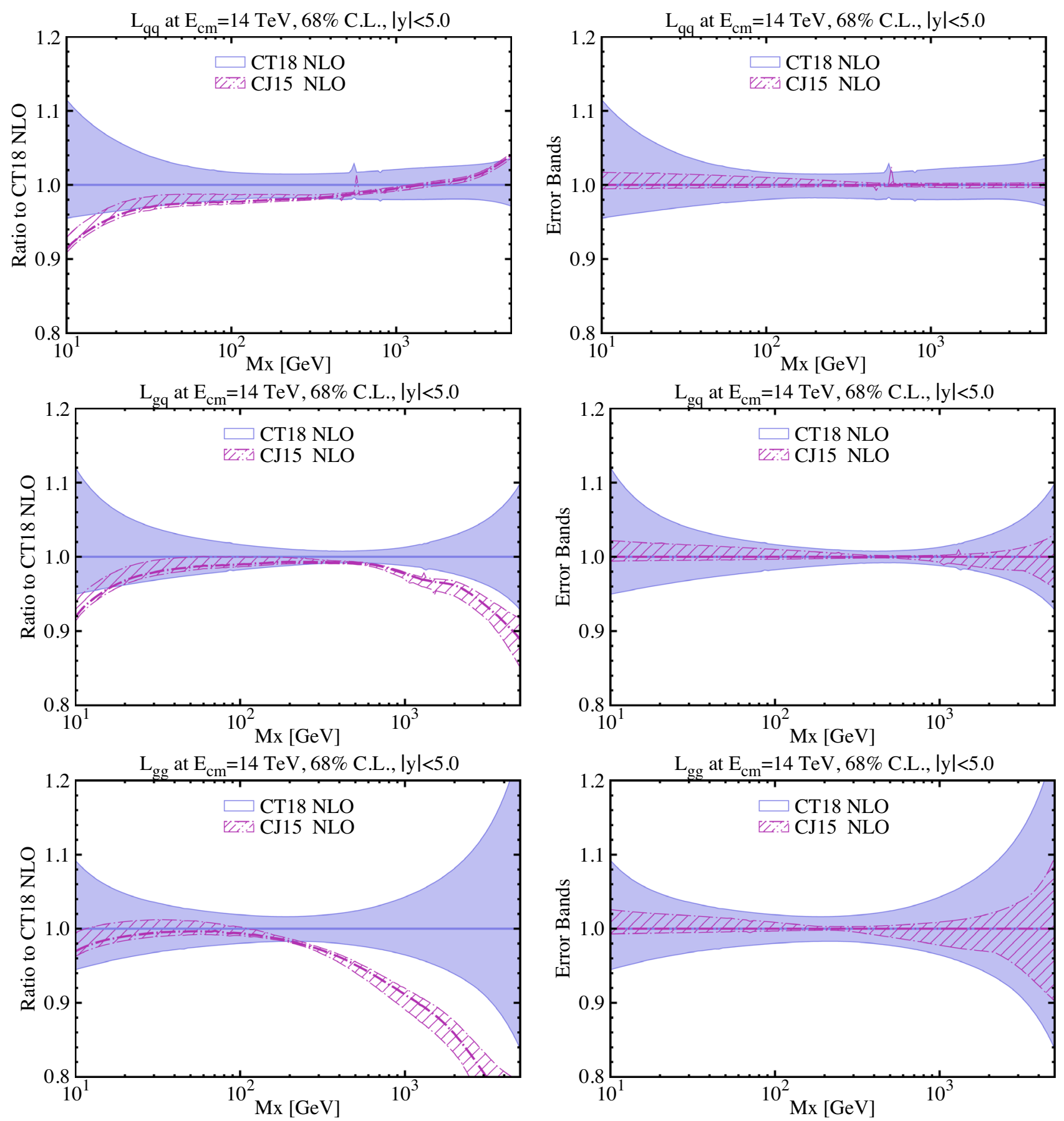

FIG. 17. Same as Fig. 16, comparing the CT18 NLO and CJ15 NLO parton luminosities.

involving gluons. In the region of the Higgs boson mass, $M_{X} \sim 125 \mathrm{GeV}$, the improvement on the $g g$ luminosity, $L_{g g}$, shown in the lowest panels of Fig. 16, is very small. In the $\mathrm{TeV}$-scale mass range, however, reductions in the PDF uncertainties of $L_{g g}$ are more sizable, closer to $\sim 20 \%$. Parton luminosities computed using CT18Z NNLO behave distinctly from CT18 in several respects. For example, in the $W / Z$ boson-mass region, the central predictions for the $q q$ luminosity are approximately 3-4\% higher in CT18Z relative to CT18. The other parton luminosities are similarly enhanced in CT18Z in the lowmass region, $M_{X} \lesssim 100 \mathrm{GeV}$, primarily because of the $x$-dependent DIS factorization scale used in CT18Z. This small- $x$ enhancement is about the same in CT18X and Z, in contrast to CT18, which more closely resembles CT14 HERAII . While the high-mass quark-quark luminosity, $L_{q q}$, is relatively unmodified in CT18Z, $L_{g q}$ and $L_{g g}$ are suppressed for $M_{X} \gtrsim 100-300 \mathrm{GeV}$; for the gluon-gluon luminosity, this suppression can be as large as $4 \%$ between $100 \mathrm{GeV}$ and $1 \mathrm{TeV}$. 

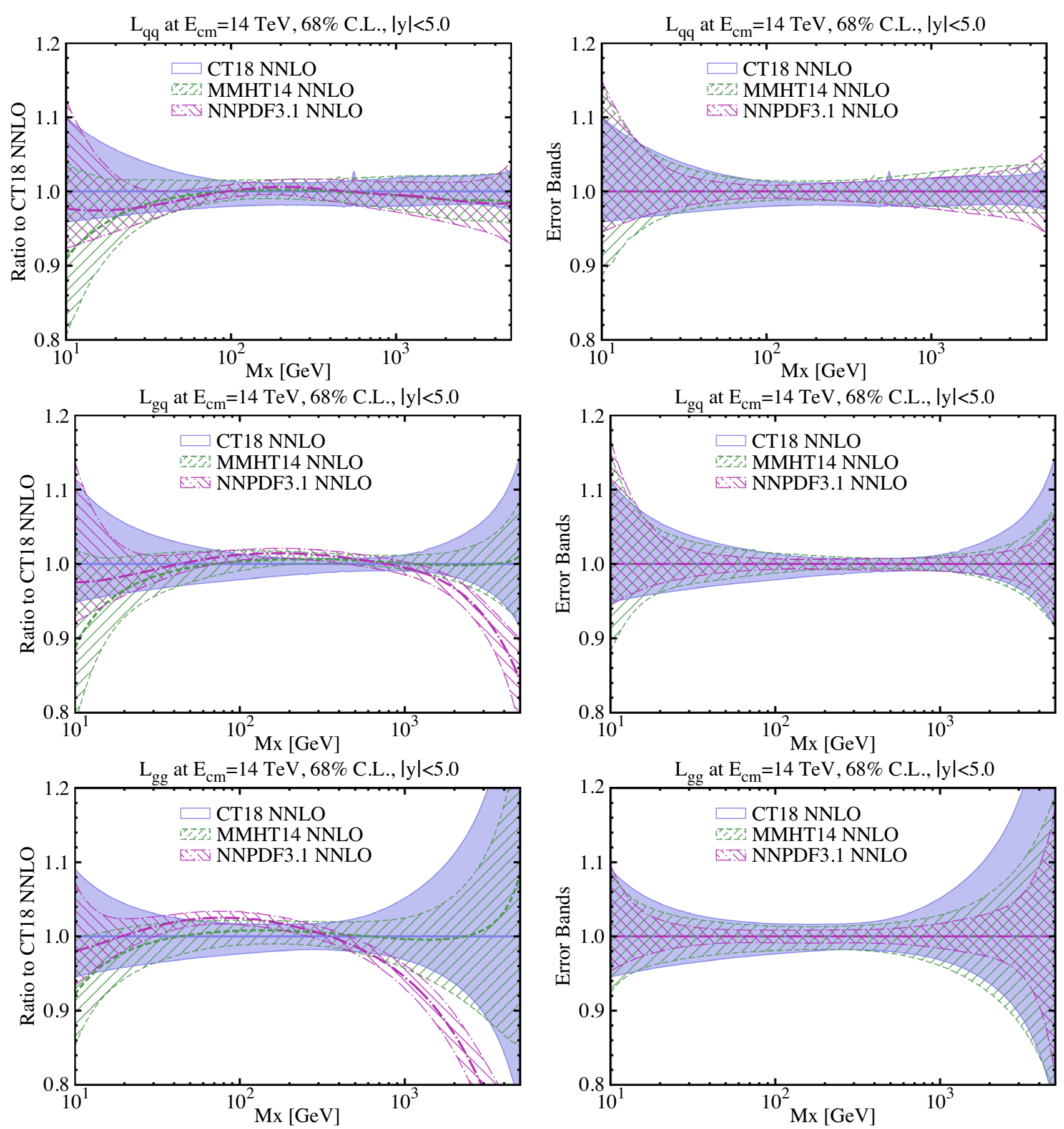

FIG. 18. Same as Fig. 16, comparing the CT18, MMHT14, and NNPDF3.1 NNLO parton luminosities with $\alpha_{s}\left(M_{Z}\right)=0.118$.

In Figs. 17 and 18, we compare these parton luminosities against those from other groups: CJ15 [5], MMHT14 [2], and NNPDF3.1 [3]. Here we adopt the rescaled 68\% C.L. for the CT18 PDFs to match the convention of the other groups. The comparison in Fig. 17 is done at NLO, because CJ15 PDFs are not available at NNLO in QCD. The PDF uncertainties on the CJ15 NLO luminosities are smaller than those on the CT18 NLO (see right insets), in part due to a smaller tolerance criterion $\left(\Delta \chi^{2}=1\right)$ and less flexible parametrization forms for the $\bar{u}$ and $\bar{d}$ PDFs employed by
CJ15. The CT18 NLO $q q$ luminosity central value is approximately $8 \%$ to $5 \%$ higher than $\mathrm{CJ} 15$ for mass values $10 \lesssim M_{X} \lesssim 2 \times 10^{3} \mathrm{GeV}$ and up to $8-10 \%$ lower for higher values (see left insets). The CT18 PDF error band for $L_{q q}$ covers that of CJ15 over the mass range $20 \lesssim M_{X} \lesssim 2 \times 10^{3} \mathrm{GeV}$. The CT18 NLO gq luminosity is approximately $2 \%$ higher than $\mathrm{CJ} 15$ in the mass region relevant for Higgs production, $100 \lesssim M_{X} \lesssim 300 \mathrm{GeV}$. It is also higher everywhere else, with major differences present at low masses, $M_{X} \lesssim 100 \mathrm{GeV}$, where it is $8 \%$ higher, and 
at high masses, $M_{X} \gtrsim 650 \mathrm{GeV}$, where differences are about $12 \%$ at $M_{X} \approx 5 \mathrm{TeV}$. For the $g g$ luminosity, CT18 at NLO is higher everywhere, in particular, differences are larger than $20 \%$ at $M_{X} \approx 5 \mathrm{TeV}$.

The luminosities obtained using CT18 NNLO PDFs are compared to those obtained with MMHT14 and NNPDF3.1 PDFs in Fig. 18. The NNPDF3.1 PDFs set is selected with $\alpha_{s}\left(M_{Z}\right)=0.118$. The central values of $q q$ and $g q$ luminosities for the three groups agree within a few percent in the mass region $100 \lesssim M_{X} \lesssim 10^{3} \mathrm{GeV}$, where they also have comparable PDF uncertainties. Comparing the NNLO $g g$ luminosities in the mass range $20<M_{X}<300 \mathrm{GeV}$, we see that MMHT14 is within a percent or so of CT18, while NNPDF3.1 is $2-3 \%$ higher. Furthermore, the uncertainty band for MMHT14 in this range is similar to that of CT18, while that of NNPDF3.1 is smaller. At larger masses $M_{X} \gtrsim 300 \mathrm{GeV}$, we observe a rapid drop of the NNPDF3.1 luminosity. Moreover, the NNPDF3.1 uncertainty is smaller over all the mass range.

In 2012-2015, several detailed studies [40,143,152] of the contemporary global PDF fits were carried out, including benchmark comparisons of their methodologies. The understanding gained from those studies has led to the 2015 recommendation on the usage of PDFs at the LHC [152]. The benchmarking also resulted in the improved agreement among the CT, MMHT, and NNPDF PDFs, which in turn allowed the PDF4LHC working group to combine these global PDFs as inputs into the widely used PDF4LHC15 PDF ensembles.

Since 2015 a great deal of LHC data has been added to the latest global fits. This has led in some cases to an increase in the differences among the central PDFs of the groups as compared to the corresponding 2015 PDF releases. This change may be attributable to various factors. In particular, small- $x$ resummation or a nonconventional choice of QCD scales in NNLO DIS cross sections modify the small- $x$ PDFs, as exemplified by CT18Z NNLO in Figs. 7 and 16. The NNPDF3.1 parton luminosity show more pronounced differences vs CT18 and MMHT2014 in some regions, cf. Fig. 18. A followup study is currently underway to better understand the impact of the LHC data and methodological choices on each global PDF.

\section{PDF moments and sum rules}

Knowledge of the integrated PDF Mellin moments has long been of interest, both for their phenomenological utility, and for their relevance to lattice QCD computations of hadronic structure $[21,153,154]$. In the case of the former, PDF moments can serve as valuable benchmarks for the purpose of comparing various global analyses and theoretical approaches, and can also be informative descriptors of the PDFs themselves. This follows especially from the fact that numerical results obtained for PDFs of a given order are connected with the $x$ dependence of the underlying parton distribution, with, in general, higher-order
Mellin moments mostly determined by the PDFs' high- $x$ behavior. In Ref. [21], an analysis of the sensitivities of HEP data to lattice-calculable quantities-specifically, the Mellin moments and parton quasidistribution functionswas performed to further develop the still-emerging PDFlattice effort $[153,154]$.

Integrated moments can in general be evaluated for practically any phenomenological PDF from its underlying distribution, provided the moment in question is convergent over the full range of support. However, in this analysis, we concentrate special attention on

$$
\left\langle x^{n}\right\rangle_{g}(Q)=\int_{0}^{1} d x x^{n} g(x, Q),
$$

with $n=1$ for the gluon, as well as

$$
\begin{aligned}
& \left\langle x^{n}\right\rangle_{q^{+}}(Q)=\int_{0}^{1} d x x^{n}[q+\bar{q}](x, Q) \text { for } n=1,3, \ldots, \\
& \left\langle x^{n}\right\rangle_{q^{-}}(Q)=\int_{0}^{1} d x x^{n}[q-\bar{q}](x, Q) \text { for } n=2,4, \ldots
\end{aligned}
$$

for the quark distributions, where we denote the charge conjugation-even (odd) quark combinations as $q^{ \pm}=q \pm \bar{q}$. We primarily consider these specific PDF moments of Eq. (12) with $n=1$ and Eq. (13) for compatibility with lattice QCD determinations, which are only able to compute the odd $(n=1,3, \ldots)$ moments of $(q+\bar{q})$-type distributions and even $(n=2,4, \ldots)$ moments for $(q-\bar{q})$-type distributions. This follows from the fact that lattice calculations extract the integrated Mellin moments from hadronic matrix elements as

$$
\frac{1}{2} \sum_{s}\left\langle p, s\left|\mathcal{O}_{\left\{\mu_{1}, \ldots, \mu_{n+1}\right\}}^{q}\right| p, s\right\rangle=2\left\langle x^{n+1}\right\rangle_{q}\left[p_{\mu_{1}} \cdots p_{\mu_{n+1}}-\text { traces }\right] .
$$

In Eq. (14), the lattice operators are $\mathcal{O}_{\left\{\mu_{1}, \ldots, \mu_{n+1}\right\}} \sim$ $i^{n} \bar{q} \gamma_{\mu_{1}} \stackrel{\leftrightarrow}{D}_{\mu_{2}} \ldots \stackrel{\leftrightarrow}{D}_{\mu_{n+1}} q$, involving covariant derivatives in such a way that successive derivative insertions increase the order of the extracted moment, but also alternate the evenness and oddness under charge conjugation.

We compute a number of the typical benchmark PDF Mellin moments using our updated CT18 and CT18Z NNLO fits and compare against the older CT14 HERAII NNLO parametrization as well as the recent MMHT14 NNLO, CJ15 NLO, and NNPDF3.1 global analyses. In all cases, moments are evaluated for an $\overline{\mathrm{MS}}$ factorization scale of $Q=2 \mathrm{GeV}$, which is also the standard matching scale computed in lattice QCD calculations. We summarize the numerical results of the PDF moment calculations in the entries of Table VI as well as in Figs. 19-20. We point out that the comparatively small values of the CJ15 NLO uncertainties are primarily attributable to the use of $\Delta \chi^{2}=1$ 
TABLE VI. We collect values of several PDF moments computed according to CT18, CT18Z, CT14 HERAII, MMHT14, CJ15 NLO, and NNPDF3.1, all at the scale $Q=2 \mathrm{GeV}$. The moments are chosen for their dual interest both as benchmarks for phenomenological calculations and relevance to lattice QCD calculations. In the descending order, we show the three lowest moments of the isovector $(u-d)$ distribution, the first moment of the gluon, the first and second moments, respectively, for the flavor-separated $u, d, s$ distributions, and two measures of light quark flavor symmetry violation: the zeroth moment of the flavor $\mathrm{SU}(2)$ difference, $\langle 1\rangle_{\bar{d}-\bar{u}}$, and the moment ratio related to the strangeness suppression, $\kappa_{s}$, defined in Eq. (19). We note that the $\bar{d}$ and $\bar{u}$ distributions are not constrained to coincide at $x \rightarrow 0$ in NNPDF3.1, leaving $\langle 1\rangle_{\bar{d}-\bar{u}}$ undefined, whereas the strange suppression factor was not fitted in CJ15. Here, all computed moment uncertainties are either based on $68 \%$ C.L., or have been rescaled accordingly for comparison.

\begin{tabular}{|c|c|c|c|c|c|c|}
\hline PDF moment & CT18 & CT18Z & CT14 $4_{\text {HERAII }}$ & МMHT14 & CJ15 & NNPDF3.1 \\
\hline$\langle x\rangle_{u^{+}-d^{+}}$ & $0.156(7)$ & $0.156(6)$ & $0.159(6)$ & $0.151(4)$ & $0.1518(13)$ & $0.152(3)$ \\
\hline$\left\langle x^{2}\right\rangle_{u^{-}-d^{-}}$ & $0.055(2)$ & $0.055(2)$ & $0.055(2)$ & $0.053(2)$ & $0.0548(2)$ & $0.057(3)$ \\
\hline$\left\langle x^{3}\right\rangle_{u^{+}-d^{+}}$ & $0.022(1)$ & $0.022(1)$ & $0.022(1)$ & $0.022(1)$ & $0.0229(1)$ & $0.022(1)$ \\
\hline$\langle x\rangle_{q}$ & $0.414(8)$ & $0.407(8)$ & $0.415(8)$ & $0.411(9)$ & $0.4162(8)$ & $0.410(4)$ \\
\hline$\langle x\rangle_{u^{+}}$ & $0.350(5)$ & $0.350(4)$ & $0.351(5)$ & $0.348(5)$ & $0.3480(6)$ & $0.348(4)$ \\
\hline$\langle x\rangle_{d^{+}}$ & $0.193(5)$ & $0.194(5)$ & $0.193(6)$ & $0.197(5)$ & $0.1962(9)$ & $0.196(4)$ \\
\hline$\langle x\rangle_{s^{+}}$ & $0.033(9)$ & $0.041(8)$ & $0.031(8)$ & $0.035(8)$ & $0.0313(2)$ & $0.039(4)$ \\
\hline$\left\langle x^{2}\right\rangle_{u^{-}}$ & $0.085(1)$ & $0.084(1)$ & $0.085(1)$ & $0.083(1)$ & $0.0853(2)$ & $0.085(3)$ \\
\hline$\left\langle x^{2}\right\rangle_{d^{-}}$ & $0.030(1)$ & $0.029(1)$ & $0.030(1)$ & $0.030(1)$ & $0.0305(2)$ & $0.028(3)$ \\
\hline$\left\langle x^{2}\right\rangle_{s^{-}}$ & $\ldots$ & $\ldots$ & $\ldots$ & $0.001(1)$ & $\ldots$ & $0.001(4)$ \\
\hline$\langle 1\rangle_{\bar{d}-\bar{u}}$ & $-0.12(35)$ & $-0.07(29)$ & $-0.37(41)$ & $0.084(15)$ & $0.103(20)$ & $\ldots$ \\
\hline$\kappa_{s}$ & $0.49(16)$ & $0.61(14)$ & $0.46(13)$ & $0.51(14)$ & .. & $0.563(82)$ \\
\hline
\end{tabular}

criterion, and, in some cases, a comparatively more restrictive parametrization.

\section{Observations}

In general, we observe concordance among the moments of the light distributions, including those of the isovector (i.e., $u-d)$ combination, $\left\langle x^{1,3}\right\rangle_{u^{+}-d^{+}}$and $\left\langle x^{2}\right\rangle_{u^{-}-d^{-}}$. Notably, the CT results for the first isovector moment, $\langle x\rangle_{u^{+}-d^{+}} \sim 0.156-0.159$, are marginally larger than those obtained under the other fits considered here, which produce $\langle x\rangle_{u^{+}-d^{+}} \sim 0.151-0.152$, but are nevertheless in close agreement at the $1 \sigma$ level. Similarly, we recover very robust agreement for the first moment of the gluon PDF, which can be understood to carry $\sim 41 \%$ of the proton's longitudinal momentum at the scale $Q=2 \mathrm{GeV}$. We find a slightly smaller total contribution to the momentum sum rule from the gluon under CT18Z NNLO, which results in $\langle x\rangle_{g}=0.407(8)$, but it is still easily in agreement within errors with the CT18 NNLO calculation, $\langle x\rangle_{g}=0.414(8)$. This is consistent with the modest reduction in the central gluon shown for CT18Z in the lower-right panel of Fig. 7.

For the contributions of the individual flavor-separated quark densities to the proton's longitudinal momentum, we again find in general strong convergence among our new global analysis and the results of previous and other fits. This is especially true for the total $u^{+}$and $d^{+}$first moments, for which we find concordance at $\langle x\rangle_{u^{+}} \sim 0.35$ and $\langle x\rangle_{d^{+}} \sim 0.193-0.194$. The situation is similar for the total
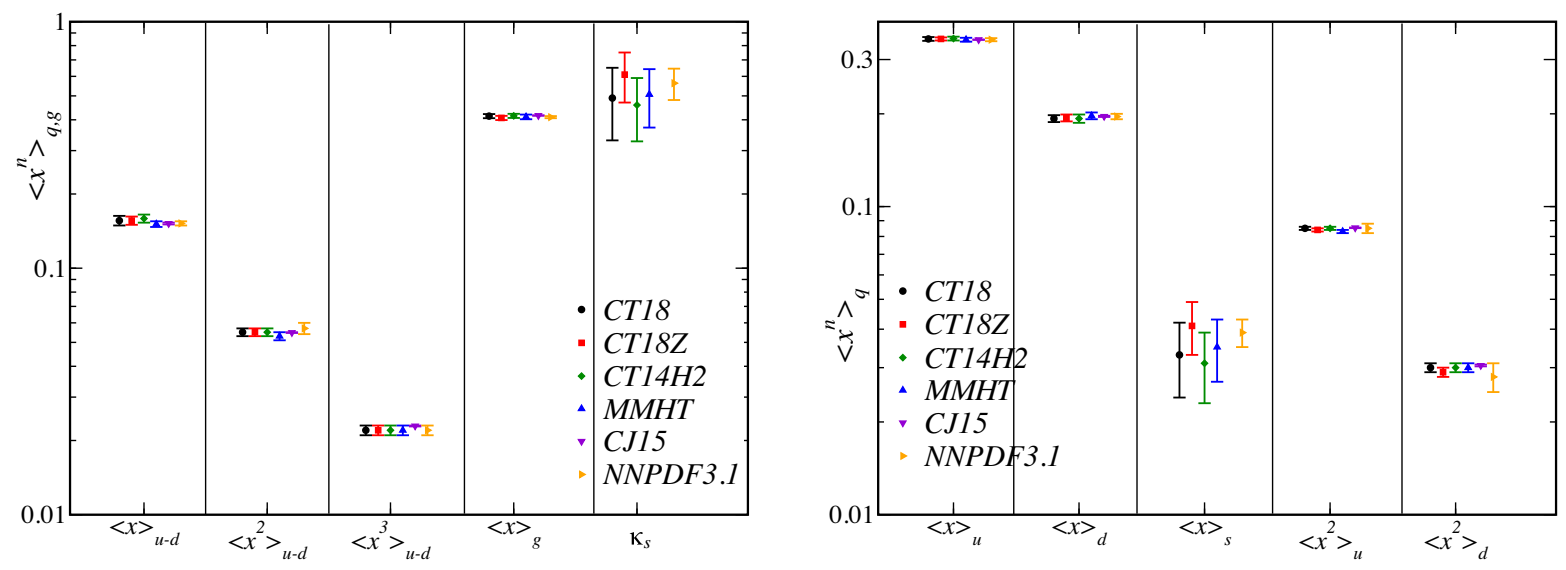

FIG. 19. A graphical comparison of the PDF moments summarized in Table VI, with the exception of the results for the zeroth moment of $\bar{d}-\bar{u}$ combination, relevant for studies of the Gottfried sum rule; this latter quantity is given in Fig. 20. The CJ15 global fit does not determine $\kappa_{s}$ and $\langle x\rangle_{s}$ as independent entities from the data, their respective predictions are not shown. 


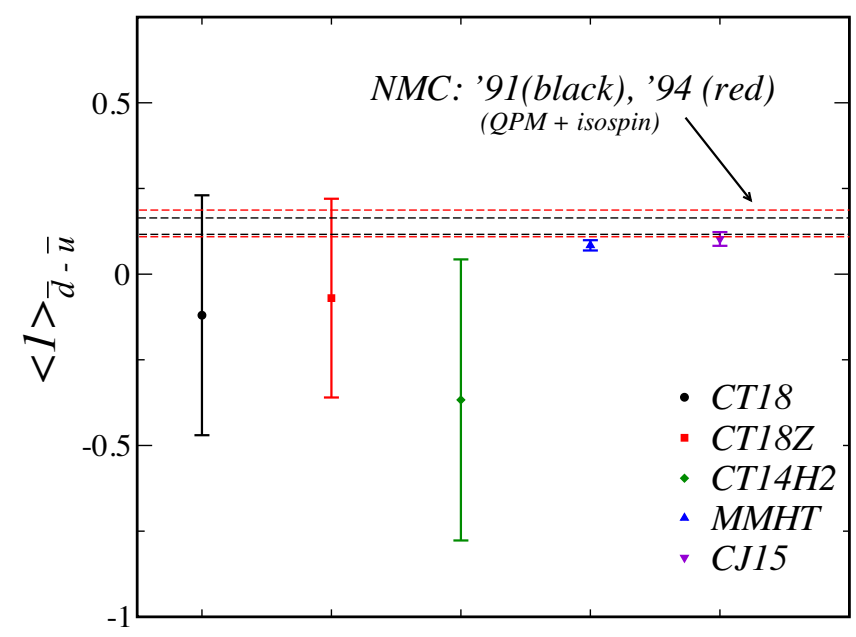

FIG. 20. A visual comparison of the results for $\int_{0}^{1} d x[\bar{d}(x)-$ $\bar{u}(x)]$. The horizontal nested black and red bands correspond to the values extracted from the original NMC analyses from 1991 [155] and 1994 [156], respectively. These were based on direct quark-parton model extractions of the flavor asymmetry PDF from the deuteron-to-proton structure function ratio measured at $Q^{2}=4 \mathrm{GeV}^{2}$ for a range of $x \lesssim 0.7$. While all but the highest $x$ bin in this dataset is consistent with CTEQ kinematical cuts, the very low $Q$ is exactly at the boundary of the $Q$ cut, and likely subject to substantial higher-twist corrections, especially for the higher $x$ bins.

nucleon strangeness momentum, but with a somewhat greater quantitative spread about $\langle x\rangle_{s^{+}}=3-4 \%$. For CT18 NNLO, we obtain $\langle x\rangle_{s^{+}}=3.3 \pm 0.9 \%$ - similar to CT14 HERAII. In shifting to CT18Z, a 24\% larger nucleon strange content is preferred, but with comparable error.

In addition to the first moments of the quark and gluon densities, $\langle x\rangle_{q^{+}, g}$, we also evaluate the second moments of select $q-\bar{q}$ quark asymmetries according to Eq. (13), finding for $\left\langle x^{2}\right\rangle_{u^{-}, d^{-}}$very close alignment among CT18(Z) and previous calculations. Recent CT fits and CJ15 do not independently parametrize $s$ vs $\bar{s}$, and we therefore omit entries in Table VI for $\left\langle x^{2}\right\rangle_{s^{-}}$.

Results on the integrated PDF moments are also of interest to phenomenological sum rules-for instance, the Gottfried sum rule [157], which relates $x^{-1}$-weighted moment of the $F_{2}^{p-n}=F_{2}^{p}-F_{2}^{n}$ structure function difference,

$\left.\int_{0}^{1} \frac{d x}{x} F_{2}^{p-n}(x, Q)\right|_{\mathrm{QPM}}=\frac{1}{3}-\frac{2}{3} \int_{0}^{1} d x[\bar{d}-\bar{u}](x, Q)$,

to flavor-symmetry violation in the light quark sea via the breaking of the $\mathrm{SU}(2)$ relation $\bar{d}=\bar{u}$. For the zeroth moment related to the Gottfried Sum Rule, we obtain $\langle 1\rangle_{\bar{d}-\bar{u}}=$ $-0.12 \pm 0.35$ in CT18 $\left(\langle 1\rangle_{\bar{d}-\bar{u}}=-0.07 \pm 0.29\right.$ under CT18Z), generally consistent with other PDF analyses. These other analyses produce narrower uncertainties for $\langle 1\rangle_{\bar{d}-\bar{u}}$, but this follows from comparatively more restrictive parametrizations in the low- $x$ region, $x \leq 0.001$. The zeroth moment is dominated by the low-x behavior of the $\bar{u}, \bar{d}$ PDFs, for which high-energy data remain relatively sparse, as can be seen in Fig. 1. NNPDF, in contrast, imposes no restriction on the relative behavior of $\bar{u}$ and $\bar{d}$ for $x \rightarrow 0$, such that $\langle 1\rangle_{\bar{d}-\bar{u}}$ is not numerically defined; the corresponding NNPDF3.1 entry is therefore left blank in Table VI and Fig. 20. CT uses a significantly more flexible parametrization for the light-quark sea (with 11 parameters for the combined $\bar{u}$ and $\bar{d}$ PDFs, compared with five parameters in MMHT14 for $\bar{d}-\bar{u}$ [2] and five parameters in CJ15 for $\bar{d} / \bar{u}$ [5]), with no constraint on the sign of $\bar{d}-\bar{u}$, as can be deduced from the $\bar{d} / \bar{u}(x, Q=1.4 \mathrm{GeV})$ ratio plot shown in the upper-left panel of Fig. 11. We therefore find that, with this flexibility in the low- $x$ region important for $\langle 1\rangle_{\bar{d}-\bar{u}}$, modern highenergy data still allow a broad range for the zeroth moment.

The CT18(Z) values for $\langle 1\rangle_{\bar{d}-\bar{u}}$ are in agreement with the moments calculated in the original 1991 and 1994 NMC analyses $[155,156]$, which we represent in Fig. 20 as the inner, black and outer, red horizontal bands for the 1991 [155] and 1994 [156] extractions, respectively. Several aspects of the original NMC analysis can be expected to underpredict the full experimental uncertainty on the Gottfried moment, but chief among these is the fact that NMC was sensitive only to the region $0.004<x<0.8$. In addition, directly matching the NMC structure function moment to $\langle 1\rangle_{\bar{d}-\bar{u}}$, as in Eq. (15), entails a leading-order quark-parton calculation, which necessarily induces corrections from missing higher orders and other QCD effects not contained in the bands of Fig. 20. Moreover, in determining the isovector structure function $F_{2}^{p-n}$ from deuteron-to-proton cross section ratios, NMC assumed a fairly restrictive parametrization to perform low- $x$ extrapolations as well as to represent the absolute deuteron structure function. For the sake of comparison, it is instructive to consider the Gottfried sum rule in the region measured by NMC, for which we find reasonable agreement between $\mathrm{CT}$ and $\mathrm{NMC}$ :

$$
\begin{aligned}
\frac{1}{3}- & \frac{2}{3} \int_{0.004}^{0.8} d x[\bar{d}-\bar{u}](x, Q=2 \mathrm{GeV}) \\
& =0.227 \pm 0.016\left(\mathrm{NMC}^{\prime} 91\right) \\
& =0.221 \pm 0.021\left(\mathrm{NMC}^{\prime} 94\right) \\
& =0.260 \pm 0.053(\mathrm{CT} 18)
\end{aligned}
$$

We stress that the relatively narrow CT uncertainty about the NMC extractions obtained for the restricted integral over $0.004<x<0.8$ underscores the importance of low- $x$ PDF uncertainties on $\bar{u}, \bar{d}$ in the still lightly probed $x<10^{-3}$ region. These must be brought under further control before phenomenological analyses of high-energy data can make a definitive statement about the violation of $\mathrm{SU}(2)$ flavor symmetry at the moment level of Eq. (15). 
TABLE VII. Like Table VI, but now comparing the most recent results obtained under CT18(Z) and CT14 $4_{\text {HERAII }}$ with a representative selection of recent lattice QCD calculations in the rightmost column. For the latter, reported results are generally taken from the recent whitepapers in Refs. $[153,154]$. The information given in this table is not exhaustive, but summary, and we refer the interested reader to the detailed presentations in Refs. [153,154] for extensive surveys of modern lattice calculations. Those lattice entries corresponding to single calculations are given with the associated reference, whereas those which result from a combination of several lattice extractions are indicated with “ $\star$." In particular, for $\langle x\rangle_{u^{+}-d^{+}}$we follow Ref. [154] in supplying ranges obtained from various calculations, grouped according to the number of active flavors, $N_{f}$, in the lattice action used. Meanwhile, the corresponding result for $\left\langle x^{2}\right\rangle_{u^{-}-d^{-}}$shown above is an average over the result in Ref. [159] and two separate calculations reported in Ref. [160].

\begin{tabular}{|c|c|c|c|c|}
\hline PDF moment & CT18 & CT18Z & CT14 $14_{\text {HERAII }}$ & Lattice \\
\hline$\langle x\rangle_{u^{+}-d^{+}}$ & $0.156(7)$ & $0.156(6)$ & $0.159(6)$ & $\begin{array}{c}0.153-0.194^{N_{f}=2+1+1} \star \\
0.111-0.209^{N_{f}=2+1} \star \\
0.166-0.212^{N_{f}=2 \star}\end{array}$ \\
\hline$\left\langle x^{2}\right\rangle_{u^{-}-d^{-}}$ & $0.055(2)$ & $0.055(2)$ & $0.055(2)$ & $0.107(98) \star$ \\
\hline$\left\langle x^{3}\right\rangle_{u^{+}-d^{+}}$ & $0.022(1)$ & $0.022(1)$ & $0.022(1)$ & $\begin{array}{c}N / A \\
0.427(92)[161]\end{array}$ \\
\hline$\langle x\rangle_{g}$ & $0.414(8)$ & $0.407(8)$ & $0.415(8)$ & $\begin{array}{c}0.482(69)(48)[162] \\
0.47(4)(11)[163]\end{array}$ \\
\hline$\langle x\rangle_{u^{+}}$ & $0.350(5)$ & $0.350(4)$ & $0.351(5)$ & $\begin{array}{c}0.359(30)[161] \\
0.307(30)(18)[162]\end{array}$ \\
\hline$\langle x\rangle_{d^{+}}$ & $0.193(5)$ & $0.194(5)$ & $0.193(6)$ & $\begin{array}{c}0.188(19)[161] \\
0.160(27)(40)[162]\end{array}$ \\
\hline$\langle x\rangle_{s^{+}}$ & $0.033(9)$ & $0.041(8)$ & $0.031(8)$ & $\begin{array}{c}0.052(12)[161] \\
0.051(26)(5)[162]\end{array}$ \\
\hline$\left\langle x^{2}\right\rangle_{u^{-}}$ & $0.085(1)$ & $0.084(1)$ & $0.085(1)$ & $0.117(18)[164]$ \\
\hline$\left\langle x^{2}\right\rangle_{d^{-}}$ & $0.030(1)$ & $0.029(1)$ & $0.030(1)$ & $0.052(9)[164]$ \\
\hline$\left\langle x^{2}\right\rangle_{s^{-}}$ & $\ldots$ & $\ldots$ & $\ldots$ & N/A \\
\hline$\langle 1\rangle_{\bar{d}-\bar{u}}$ & $-0.12(35)$ & $-0.07(29)$ & $-0.37(41)$ & $\ldots$ \\
\hline$\kappa_{s}$ & $0.49(16)$ & $0.61(14)$ & $0.46(13)$ & $0.795(95)[158]$ \\
\hline
\end{tabular}

We may extend the analysis of the $\bar{d} \neq \bar{u}$ breaking to the $\mathrm{SU}(3)$ sector, by analyzing the ratio of the first moments of the distributions appearing in Eq. (10) leading to the strange suppression factor moment ratio,

$$
\kappa_{s}(Q) \equiv \frac{\langle x\rangle_{s^{+}}}{\langle x\rangle_{\bar{u}}+\langle x\rangle_{\bar{d}}},
$$

as illustrated in Fig. 19. The final row of Table VI lists the numerical results for this quantity for the PDF parametrizations considered above, with the exception of CJ15, which sets $R_{s}(x, Q)$ to a constant, making $s^{+}(x, Q)$ proportional to $\bar{u}(x, Q)+\bar{d}(x, Q)$. Up to uncertainties, the moments we compute are generally consistent with the traditional strangeness suppression scenario, $\kappa_{s}=0.5$. In moving from CT14 $4_{\text {HERAII }}$ to CT18, there is a modest enhancement, at $Q=2 \mathrm{GeV}$, in the preferred central value and related growth of the associated uncertainty, which shifts from $\kappa_{s}\left(\mathrm{CT} 14_{\text {HERAII }}\right)=0.46 \pm 0.13$ to $\kappa_{s}(\mathrm{CT} 18)=$ $0.49 \pm 0.16$, in very close agreement with MMHT14, in particular. The inclusion of the ATLAS $W, Z$ production data, as well as other changes leading to CT18Z, noticeably increase the ratio to $\kappa_{s}(\mathrm{CT} 18 \mathrm{Z})=0.61 \pm 0.14$ and marginally contract its uncertainty, as compared to CT18, making the value more similar to the one in NNPDF3.1. Recently, a first lattice calculation of $\kappa_{s}$ was reported by the $\chi \mathrm{QCD}$ collaboration in Ref. [158], which found $\kappa_{s}(Q=2 \mathrm{GeV})=$ $0.795 \pm 0.079$ (stat) \pm 0.053 (sys). Indeed, while this result lies just beyond the upper periphery of the values preferred by typical phenomenological fits, $\kappa_{s} \sim 0.5$, it agrees at the $1 \sigma$ level with the CT18Z result that follows from the inclusion of $7 \mathrm{TeV}$ inclusive $W, Z$ production data taken by ATLAS.

This, as well as other entries for the PDF moments determined on the QCD lattice as listed in the rightmost column of Table VII, have historically shown a general tendency to overestimate the values extracted phenomenologically. More recent lattice calculations have in some cases begun to approach the phenomenological momentse.g., for the isovector $u-d$ moments, or for the total $u$, $d$-quark and gluon momenta, $\langle x\rangle_{u^{+}, d^{+}, g}$-for which the lattice uncertainties are also sufficiently large as to allow agreement with global analyses.

Schematically, the PDF moments are extracted on the lattice from the ratio of three-point to two-point correlation functions $[153,165]$ :

$R(t, \tau, \mathbf{p}, \hat{O})=\frac{\sum_{a, b} \Gamma_{b, a}\left\langle B_{a}(t, \mathbf{p})|\hat{O}(\tau)| B_{b}(0, \mathbf{p})\right\rangle}{\sum_{a, b} \Gamma_{b, a}\left\langle B_{a}(t, \mathbf{p}) \mid B_{b}(0, \mathbf{p})\right\rangle}$,

where the $B_{a, b}$ are baryon interpolating operators, $t$ the source-sink Euclidean time separation, and $\tau$ the Euclidean 
time associated with the operator $\hat{O}$ insertion noted in Eq. (14). For the lower moments of the nucleon parton distributions, the lattice output is substantially governed by the interplay between excited-state contamination of the correlation functions, which in general depend on Euclidean time as $\sim \exp \left(-m_{i} t\right)$, and the lattice signal-tonoise ratio, which goes as $S / N \sim \exp \left(-\left(E_{N}-[3 / 2] m_{\pi}\right) t\right)$. As such, lattice calculations at physical pion mass (or chiral extrapolations thereto) lead to more rapid deterioration of the signal-to-noise at precisely the larger lattice times at which contributions from nucleon excited states are relatively suppressed. The subtle relationship between these lattice effects (in addition to other systematic artifacts) complicate any straightforward interpretation of the presently large or small lattice results for the PDF moments shown in Table VII.

\section{DESCRIPTION OF INDIVIDUAL DATASETS}

The CT18 global analysis includes a wide range of data from run-1 of the LHC, in addition to the extensive collection of data used in the previous CT14 analysis with the combined HERA measurements. Section II B and Tables I-II reviewed the CT18(Z) datasets and broadly summarized the overall quality of the fits in terms of $\chi^{2} / N_{p t}$ and effective Gaussian variables $S_{E}$ provided for each fitted experiment. A successful fit of the global data, however, requires a far more fine-grained exploration of the degree to which individual experiments are described well. It is important to quantitatively evaluate the agreement between data and theory with a rigorous battery of statistical measures and tests [37], including a comprehensive survey of potential tensions in fitting various experiments. We survey the landscape of experimental constraints in Sec. VA, concentrating primarily on the complementary techniques of LM scans and sensitivity calculations to elucidate the level of agreement within the fit and remaining sources of systematic tension. Section V B concentrates on the theoretical description of specific fitted experiments, while Sec. V C examines the role of NLO electroweak corrections in describing the fitted data.

Procedurally, fitting in the CT approach is done as described in Appendix B. First, we minimize the difference between data and theory by computing the best-fit values of the nuisance parameters $\lambda$ associated with the correlated systematic errors of each experiments. Then, we minimize $\chi^{2}$ with respect to the parameters $a$ of the functional forms of the parton distribution functions. We arrive at the best-fit $\chi^{2}$ given by Eq. (2) as the sum of $\left(D_{i}^{s h}\left(a_{0}\right)-T_{i}\left(a_{0}\right)\right)^{2} / s_{i}^{2}$ and squares of optimal individual nuisance parameters $\bar{\lambda}\left(a_{0}\right)$. Here $T_{i}$ is the $i$ th theory prediction, $D_{i}^{s h}$ denotes the respective data value shifted by the optimal systematic displacements of the nuisance parameters; $s_{i}$ is the published estimate for the total uncorrelated error.
In a high-quality fit, deviations of theory from data are consistent with random fluctuations associated with statistical and systematic uncertainties [37]. To check that this is the case, we may plot the shifted data points $D_{i}^{s h}$ and the theory values $T_{i}$ for each fitted experiment. The error bars for the shifted data are the uncorrelated errors $s_{i}$ only, because the correlated systematic errors are already accounted for in the nuisance parameter values.

There is also a second comparison that needs to be considered: a histogram plotting optimal nuisance parameter values $\bar{\lambda}_{\alpha}(a)$, associated with the sources of systematic uncertainties. The nuisance parameters, which are used to model the relation between the true and the experimentallydetermined values of observables, are usually assumed to be sampled from a normal distribution $\mathcal{N}(0,1)$ with the mean equal to 0 and standard deviation equal to 1 . Thus, if too many best-fit parameters $\bar{\lambda}_{\alpha}(a)$ are far from zero according to $\mathcal{N}(0,1)$, we should be concerned. On the other hand, the situation where many $\bar{\lambda}_{\alpha}(a)$ are close to zero, meaning that the empirical histogram is narrower than $\mathcal{N}(0,1)$, is common for several new datasets that have published large numbers of systematic uncertainties. This situation is generally less of a concern, as there may be benign reasons for having too many $\bar{\lambda}_{\alpha}(a)$ that are very small, see Sec. IV.E in [37].

\section{A. Overall agreement among experiments}

\section{Revisiting effective Gaussian variables}

Let us first return to Fig. 4 illustrating the overall quality of individual description of experiments in the CT18 NNLO global fit based on the information collected in Tables I and II. Instead of examining $\chi_{E}^{2}\left(N_{p t, E}\right) / N_{p t, E}$ for individual experiments $E$, which have different probability distributions dependent on $N_{p t, E}$, we plot equivalent information in the form of a histogram of the effective Gaussian variables $S_{E}=\sqrt{2 \chi_{E}^{2}}-\sqrt{2 N_{p t, E}-1}$ listed in Tables I and II [38].

If all deviations of theory from data are purely due to random fluctuations, one would expect to recover an empirical distribution of $S_{E}$ that is close to $\mathcal{N}(0,1)$ for any $N_{p t, E}$. In practice, any recent global fit renders an $S_{E}$ distribution that is statistically incompatible with $\mathcal{N}(0,1)$ [37], indicating that too many experiments are underfitted or overfitted compared to the textbook case.

For the CT18 NNLO fit, the observed $S_{E}$ distribution shown in Fig. 4 is most compatible with $\mathcal{N}(0.6,1.9)$. The probability that is compatible with $\mathcal{N}(0,1)$ is very small ( $p=2.5 \times 10^{-5}$ according to the Anderson-Darling test [37]). In the figure, we labeled the experiments with the largest deviations from $S_{E}=0$. These are the combined HERAI + II dataset on inclusive DIS [30] with $S_{E} \approx 5.7$, which provides the dominant constraints on the PDFs and must be retained in the global analysis despite the 

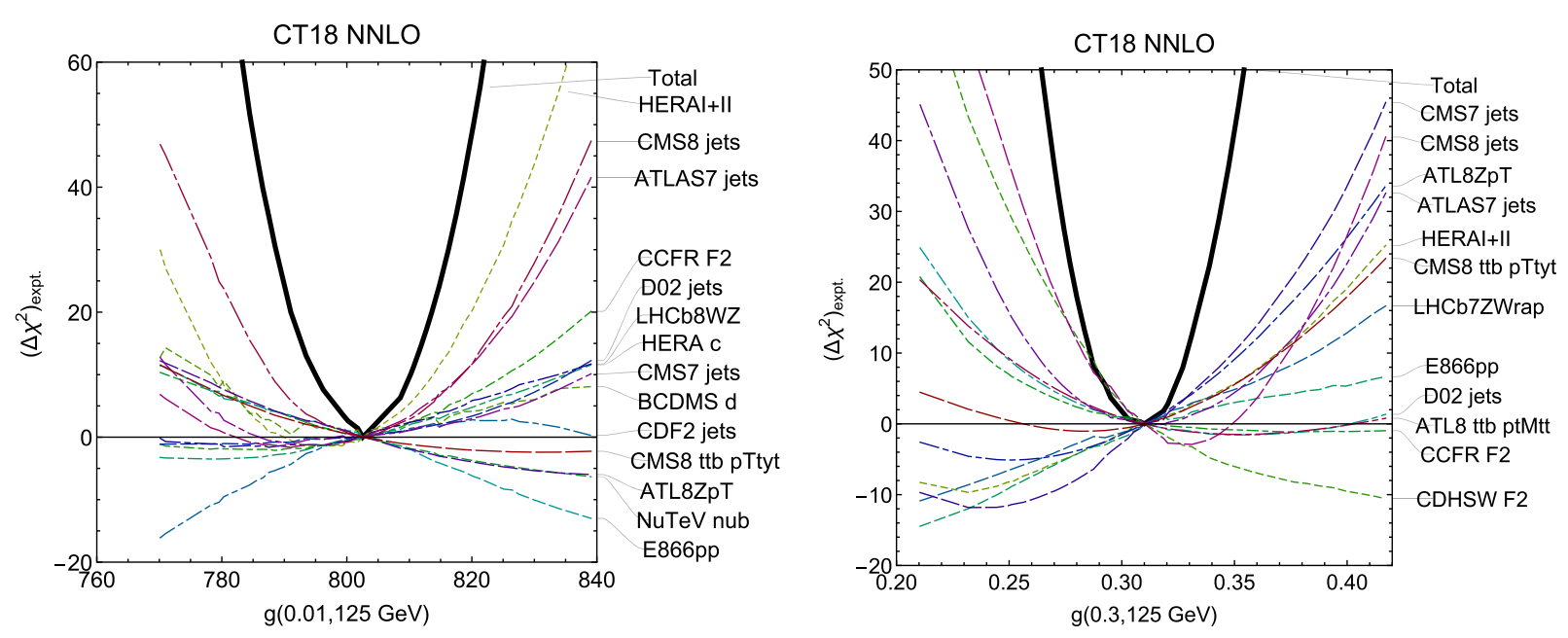

FIG. 21. LM scans for the gluon PDF at $Q=125 \mathrm{GeV}$ and $x=0.01$ and 0.3 , based upon the CT18 NNLO fits.

quality-of-fit issues discussed in Sec. II A 2, and the CCFR measurement [62] of the structure function $x_{B} F_{3}\left(x_{B}, Q\right)$ in charged-current DIS on iron, which has an unusually low $\chi^{2} / N_{p t} \approx 0.4$ for the central fit, but does constrain the PDF uncertainty for some flavors, as can be seen, e.g., in the LM scans presented in the next section.

We also note that the new LHC run-1 datasets, indicated by the light green color in Fig. 4, have more positive than negative $S_{E}$ values, indicating that their $\chi^{2}$ values are larger than would be expected from random fluctuations consistent with the published experimental errors, as can be verified by consulting Table II.

Two squares and two stars indicate the $S_{E}$ values for the $\mathrm{NuTeV}$ dimuon and CCFR dimuon data, respectively, which we highlight for special attention given the importance of these data for probing the strangeness PDF. An analogous plot for the alternative CT18Z fit in Fig. 59 shows increased $S_{E}$ values for the CCFR and NuTeV experiments, as compared to the CT18 fit, because of the conflicting pull of the ATLAS $7 \mathrm{TeV} W / Z$ production data.

\section{Lagrange multiplier scans}

The LM scan technique, which was introduced in Ref. [26], is among the most robust methods of assessing the level of tension in a global fit. This method involves constraining a particular fitted distribution to hold a chosen numerical value by means of Lagrange multipliers, while refitting the rest of the PDF parameters with this constraint in place. A PDF at a chosen $x$ and $Q$ can then be systematically varied away from its value preferred in an unconstrained global fit. The profile of increases in $\chi^{2}$ (or $S_{E}$ ) as a result of this variation can be computed for each fitted experiment, revealing the extent to which numerical alteration of the PDFs is connected to the ability to successfully describe specific data.

A collection of panels in Figs. 21-25 demonstrates $\chi^{2}$ profiles in LM scans for a broad range of CT18 NNLO
PDFs, typically at a high scale $Q=100 \mathrm{GeV}$ relevant for high-energy processes, and for select parton fractions representative of the PDF behavior at low $x(x=0.002$ and 0.023) and high $x(x=0.1$ and 0.3). Among the generic features of the scans, we observe that, while the global $\chi^{2}$ for all experiments is close to parabolic in well-constrained $(x, Q)$ regions, some individual experiments may prefer the PDF values that are quite different from the global minimum. At the global minimum itself, the $\chi_{E}^{2}$ for such an experiment may be elevated by up to tens of units.

In Fig. 21, for instance, we show two LM scans associated with the gluon density, $g(x, Q)$. In the left panel, the LM scan probes the pulls of the most sensitive measurements to the Higgs-region gluon PDF, which contributes to Higgs boson production through the predominant $g g \rightarrow H$ channel, especially in the neighborhood of $x=m_{H} /(14 \mathrm{TeV}) \sim 0.01$ and for $Q \sim m_{H}$. Evidently, most constraints arise due to HERA inclusive DIS data as well as the LHC jet data.

In the right-hand plot for $x=0.3$, strong constraints spread over more datasets, notably from high- $p_{T} Z$ boson $p_{T}$ and top-quark production. In particular, while the ATLAS $7 \mathrm{TeV}$ inclusive jet data prefer $g(0.3,125 \mathrm{GeV}) \approx$ 0.3 , consistent with the central value of the full fit, the CMS 7 and $8 \mathrm{TeV}$ jet production prefer $g(0.3,125 \mathrm{GeV})=$ $0.242_{-0.020}^{+0.016}$ and $0.327_{-0.010}^{+0.015}-\mathrm{a} \approx 3 \sigma$ difference according to the $\Delta \chi^{2}=1$ criterion.

We notice that in some situations, when a significant tension between the experiments is revealed, as in the righthand plot of Fig. 21, a Hessian estimate based on the dynamic tolerance [166] may result in a much narrower PDF uncertainty than the estimate based on the total $\chi^{2}$ in the LM scan, as a consequence of the trade-off between the opposite pulls on the PDF exerted by the conflicting experiments. We discuss this further in Appendix A 4 b, with a specific example shown in Fig. 69. 

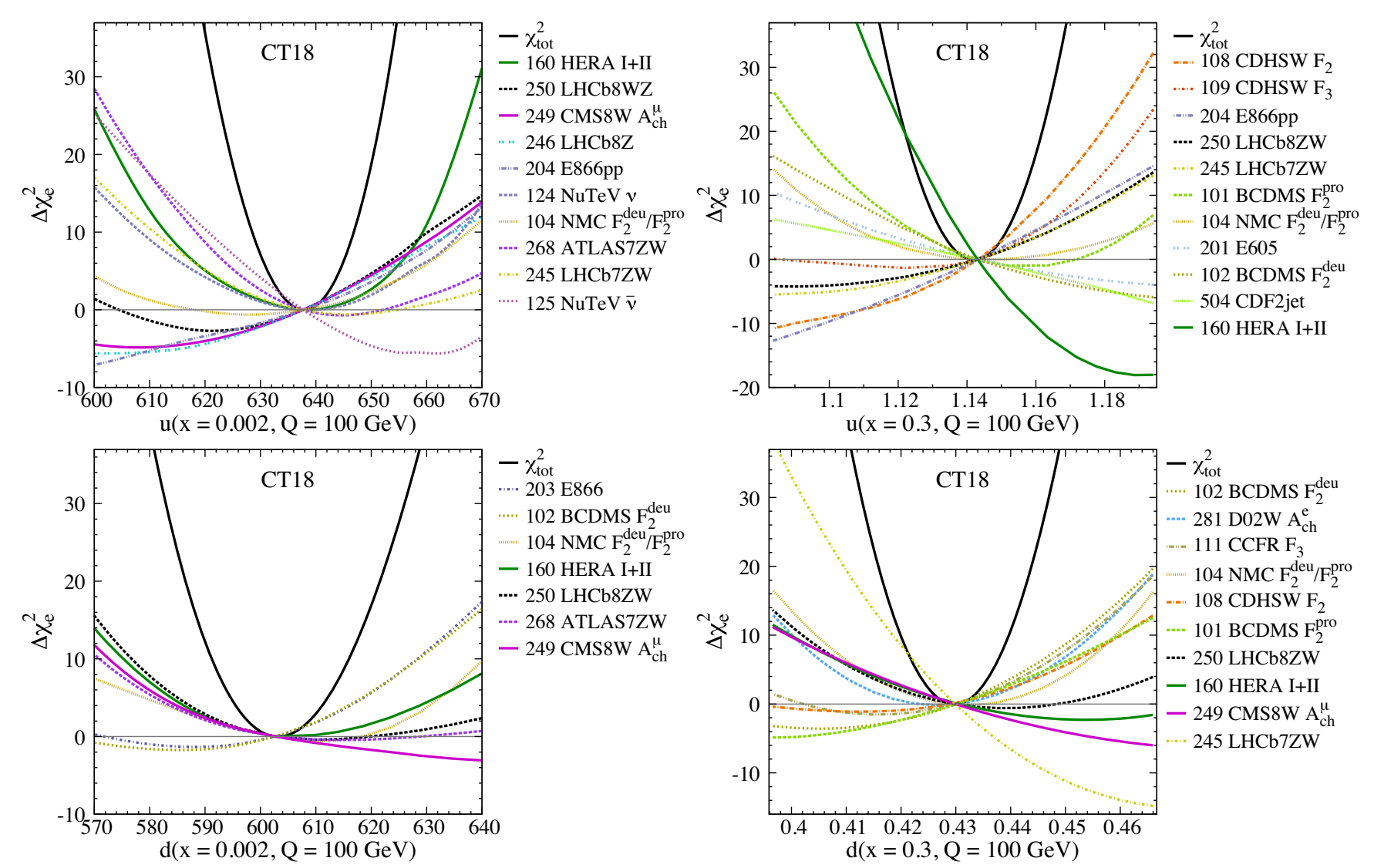

FIG. 22. LM scans for the up- and down-quark PDF at $Q=100 \mathrm{GeV}$ and $x=0.002$ and 0.3 , based upon the CT18 fits.

In Fig. 22 we show LM scans for the $u$ - and $d$-quark PDFs at $x=0.002$ and 0.3 . For the low- $x$ values, constraints from LHC $W$ and $Z$ boson data (from the LHCb, CMS, and ATLAS collaborations) stand out as expected, in addition to constraints from HERA and NuTeV. At $x=0.3$, several fixed target experiments, e.g., CDHSW, BCDMS, and E866 make significant contributions. The situations are similar for the $d$-quark density as well as for the $d / u$ ratio shown in Fig. 25.

For the $\bar{u}$ and $\bar{d}$ antiquarks in Fig. 23, as well as the $\bar{d} / \bar{u}$ ratio in Fig. 25, the LHCb data and the CMS $W$ boson charge asymmetry data play an important role at small $x$, as can be seen from Fig. 23. On the other hand, at large $x$, the flavor separation depends on the E605, E866, and NMC deuteron data.

The power of the LM method is most explicitly demonstrated by the scans on the strange quark PDF for CT18 in the third row of Fig. 23, and the strangeness ratio $R_{s}(x, Q)$ defined in Eq. (10) and scanned at $x=0.023$ and $x=0.3$ in Fig. 24. We see from the lower left inset of Fig. 23 at $x=0.002$ that the CT18 dataset provides no substantial direct constraint on $s(x, Q)$ at $x<0.01$. Rather, the behavior of $s(x, Q)$ is weakly constrained by the low-luminosity ATLAS $7 \mathrm{TeV} W$ and $Z$ data (Exp. $f=268$ ), as well as by the low- $x$ extrapolation of the constraints by the $\mathrm{NuTeV}$ and CCFR dimuon data probing $x$ above 0.01 .

At $x=0.01-0.1$, the $R_{s}$ ratios in Fig. 24 indicate the dominance of constraints from $\mathrm{NuTeV}$ and CCFR dimuon production, together with HERA inclusive DIS, with weaker constraints from $\mathrm{LHCb} W / Z$ production and the fixed-target experiments BCDMS, CDHSW, E866, and NMC. Here, the scans reveal a salient feature, that the fits using the CT18 strangeness parametrization become unstable when $R_{s}(x, Q)$ is forced to be close to 1 at $x>0.01$. For such increased $R_{s}$ values, the $\chi^{2}$ values fluctuate, or the fits fail to converge. Somewhat larger values of $R_{s}$ are tolerated at $x<0.01$.

Finally, going back to $s(x, Q)$ at $x=0.3$ in the lower right inset of Fig. 23, the very large- $x$ behavior is again determined by the extrapolation of the strangeness PDF from lower $x$, where it is constrained by the combination of the experiments listed in the figure.

We see from this section that the advantage of the Lagrange multiplier approach lies in its systematic, robust nature, as well as its ability to reveal tensions or instabilities that may be missed by the other techniques. On the other hand, this calculation requires repeated refits of the PDFs for many values of the LM parameter(s) - a limitation that makes the LM scans computationally expensive. 

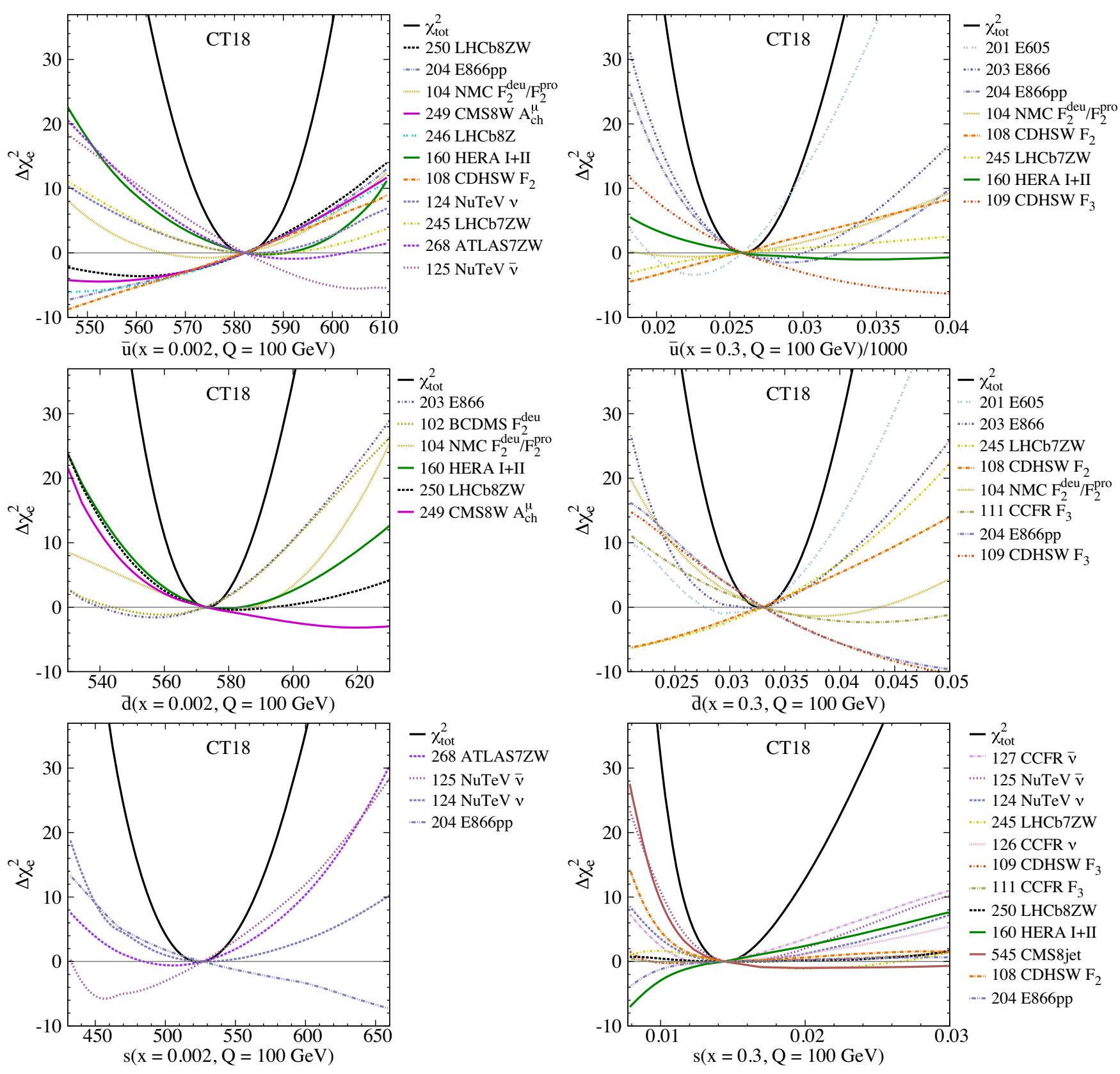

FIG. 23. Like Fig. 22, here giving LM scans for the $\bar{u}$-, $\bar{d}$-, and $s$-quark PDFs in CT18.

\section{The PDF sensitivity analysis}

A technique complementary to the LM scans explored in Sec. VA 2 is the calculation of the $L_{2}$ sensitivity. The $L_{2}$ sensitivity was first introduced in Ref. [21] for the purpose of analyzing the interplay among the pulls of the CT18(Z) data upon the fitted PDFs. Here we will review its essential definition. A closely related implementation, based on the $L_{1}$ sensitivity detailed in [20] and realized in the PDFSENSE program, will be used at the end of this section to rank the experiments of the CT18 dataset according to the sensitivity to various combinations of PDFs.
While the LM scans offer the most robust approach for exploring possible tensions among fitted datasets in a given analysis, they are very computationally costly to evaluate and done for specific choices of $x$ and $Q$. As we explain here, the $L_{2}$ sensitivity can be rapidly computed and provides a strong approximation to the $\Delta \chi^{2}$ trends in a given global analysis. Moreover, the $L_{2}$ sensitivity can be readily calculated across a wide range of $x$, allowing the $\Delta \chi^{2}$ variations shown in the LM scans to be visualized and interpreted for multiple $x$ at once. We stress that the qualitative conclusions revealed by consideration of the $L_{2}$ sensitivities, discussed and presented below, are consistent 


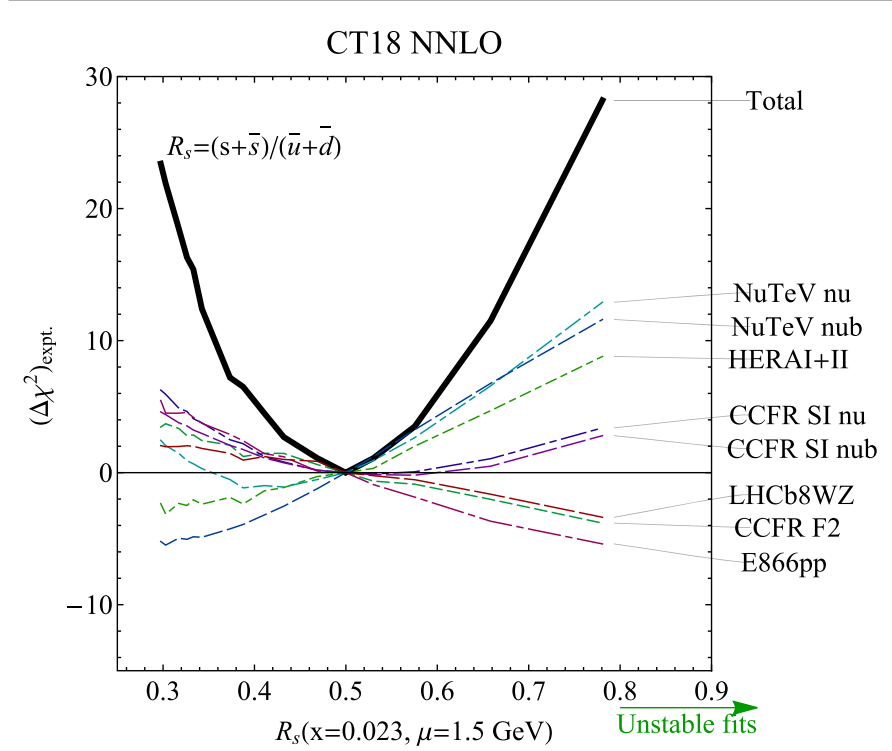

(a)

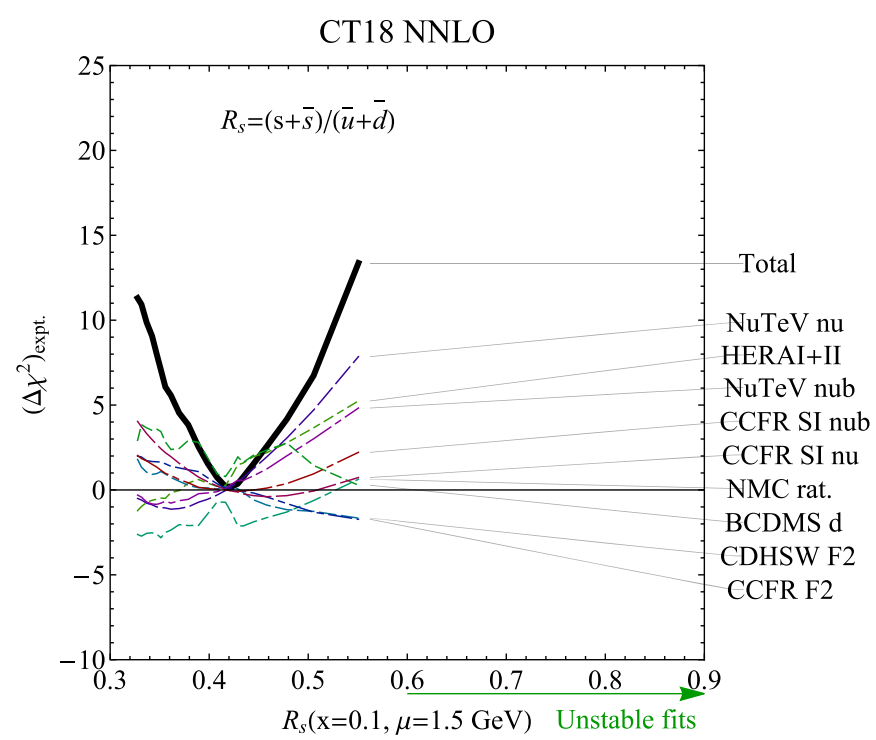

(b)

FIG. 24. The LM scan over $R_{s}$ at $Q=1.5 \mathrm{GeV}$, with $x=0.023$ and $x=0.1$, respectively, for the CT18 NNLO fit.

with the picture based on the LM scans themselves. Although the $L_{2}$ sensitivities may not always provide the same numerical ordering as the LM scans for the subdominant experiments, they offer complementary information over broader reaches of $x$ that are not completely captured by the LM scans.

We work in the Hessian formalism [22,23,91] and compute the $L_{2}$ sensitivity $S_{f, L 2}(E)$ for each experiment, $E$, as
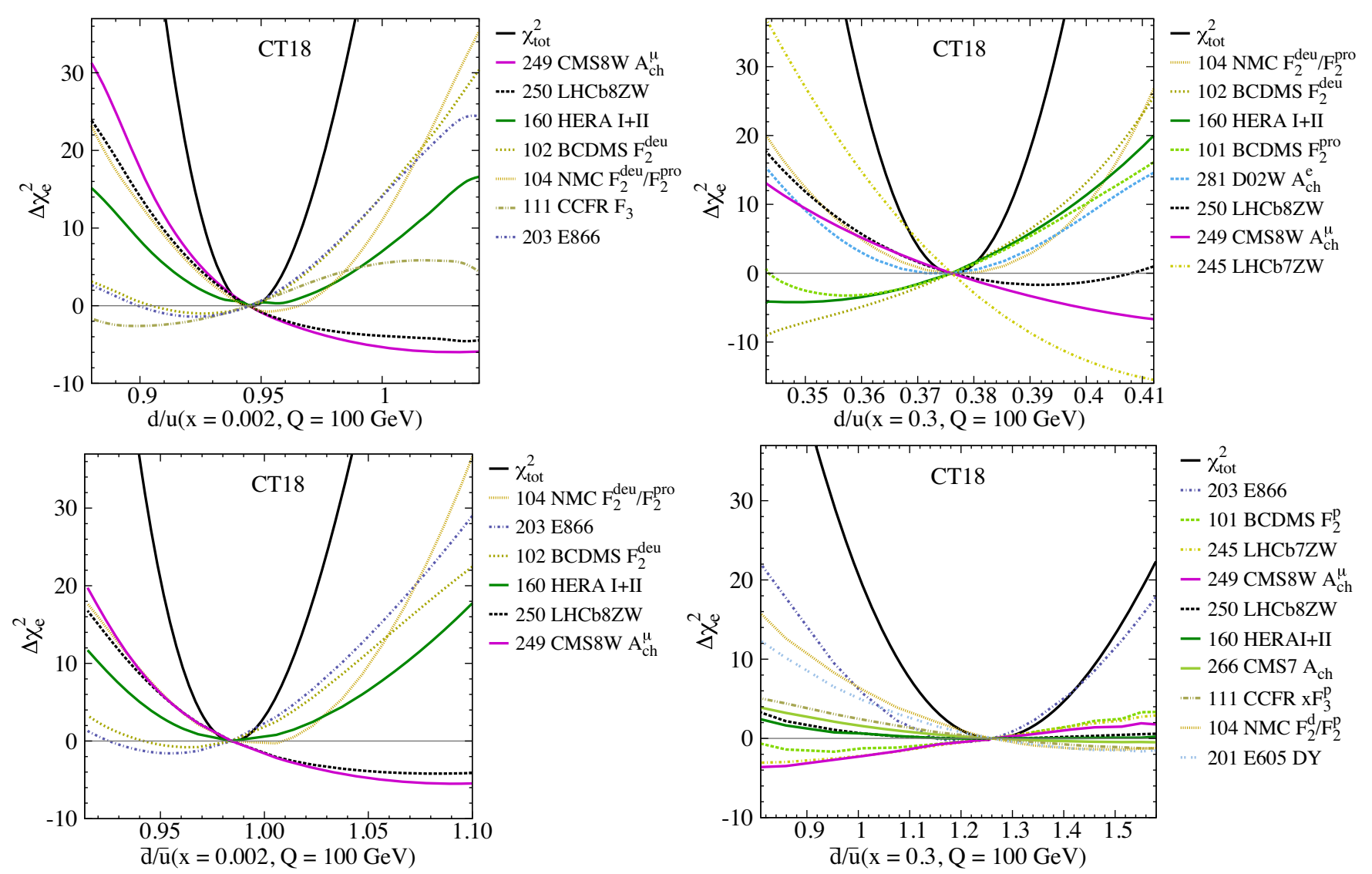

FIG. 25. Like Fig. 22, for LM scans over the ratios $d / u$ and $\bar{d} / \bar{u}$. 


$$
S_{f, L 2}(E)=\vec{\nabla} \chi_{E}^{2} \cdot \frac{\vec{\nabla} f}{|\vec{\nabla} f|}=\Delta \chi_{E}^{2} \cos \varphi\left(f, \chi_{E}^{2}\right),
$$

which yields the variation of the log-likelihood function $\chi_{E}^{2}$ due to a unit-length displacement of the fitted PDF parameters away from the global minimum $\vec{a}_{0}$ of $\chi^{2}(\vec{a})$ in the direction of $\vec{\nabla} f$. The PDF parameters $\vec{a}$ are normalized so that a unit displacement from the best fit in any direction corresponds to the default confidence level of the Hessian error set ( $90 \%$ for CT18, on average corresponding to slightly less than $\Delta \chi_{\text {tot }}^{2}=100$ in a given direction.)

This displacement increases the $\operatorname{PDF} f(x, Q)$ by its Hessian PDF error $\Delta f$, and, to the extent its PDF variation is correlated with that of $\chi_{E}^{2}$ through the correlation angle

$$
\varphi\left(f, \chi_{E}^{2}\right)=\cos ^{-1}\left(\frac{\vec{\nabla} f}{|\vec{\nabla} f|} \cdot \frac{\vec{\nabla} \chi_{E}^{2}}{\left|\vec{\nabla} \chi_{E}^{2}\right|}\right),
$$

it changes $\chi_{E}^{2}$ by $\Delta \chi_{E}^{2}\left(\hat{a}_{f}\right)=\Delta \chi_{E}^{2} \cos \varphi\left(f, \chi_{E}^{2}\right)=S_{f, L 2}(E)$. The $L_{2}$ sensitivity, $S_{f, L 2}(E)$, therefore quantifies the impact variations of PDFs at fixed $x$ and $Q$ have upon the description of fitted datasets. Plotting $S_{f, L 2}(E)$ against $x$ yields useful information regarding the pulls of the CT18 (Z) datasets upon PDFs (and PDF combinations) fitted in the global analysis. This also permits the rapid visualization of possible tensions within the global fit, since the PDF variation of some parton densities of given flavor are correlated with the variation of $\chi_{E}^{2}$ (i.e., $S_{f, L 2}(E)>0$ ), while others are anticorrelated $\left(S_{f, L 2}(E)<0\right)$, at the same values of $(x, Q)$.

The terms on the right-hand side of Eq. (21) for $S_{f, L 2}$ are computed as

$$
\Delta X=|\vec{\nabla} X|=\frac{1}{2} \sqrt{\sum_{i=1}^{N_{\text {eig }}}\left(X_{i}^{(+)}-X_{i}^{(-)}\right)^{2}},
$$

and

$\cos \varphi=\frac{\vec{\nabla} X \cdot \vec{\nabla} Y}{\Delta X \Delta Y}=\frac{1}{4 \Delta X \Delta Y} \sum_{i=1}^{N_{\mathrm{eig}}}\left(X_{i}^{(+)}-X_{i}^{(-)}\right)\left(Y_{i}^{(+)}-Y_{i}^{(-)}\right)$,

from the values $X_{i}^{(+)}$and $X_{i}^{(-)}$that a quantity $X$ takes for the parameter displacements along the $( \pm)$ direction of the $i$ th eigenvector. With these symmetric master formulas, the sum of $S_{f, L 2}(E)$ over all experiments $E$ should be within a few tens from zero, since the tolerance boundary for the total $\chi^{2}$ is close to being spherically symmetric. The $S_{f, L 2}(E)$ variables for individual experiments tend to cancel among themselves to this accuracy; the order of magnitude of $S_{f, L 2}(E)$ can be also interpreted as a measure of tension of $E$ against the rest of the experiments.

The $L_{2}$ sensitivity can be computed for individual data point residuals or optimal nuisance parameters, i.e., for parts of Eq. (2). A related, similarly informative, definition of sensitivity [20] is computed using the absolute values of residuals, $\left|r_{i}\right|$, rather than their squares $r_{i}^{2}$ (using the $L_{1}$ norm instead of the $L_{2}$ norm).

An extensive collection of the $L_{2}$ sensitivity plots for CT18(Z) PDFs and PDF ratios, reflecting the interplay and competing pulls among the CT18 datasets, can be viewed at [167]. Analogous calculations are shown for the alternative CT18Z fit in Sec. A 4.

In Fig. 26, we show the $L_{2}$ sensitivity of the CT18 data on the gluon PDF at fixed $Q=100 \mathrm{GeV}$, plotting curves for those experiments that satisfy $\left|S_{f, L 2}(E)\right| \geq 4$ for any value of $x$. This criterion generally identifies the leading five to ten experiments with strongest pulls on the PDF in the kinematical region under consideration. By its proximity to the Higgs mass scale, $Q=100 \mathrm{GeV}$, Fig. 26 highlights the opposing pulls of a number of CT18 datasets relevant for the $14 \mathrm{TeV}$ Higgs boson production cross section, $\sigma_{H}(14 \mathrm{TeV})$, and is the $L_{2}$-based counterpart to Fig. 21 (left). In addition to several non-LHC experiments (Exp. IDs $=101,102,108,109,160,204)$ imposing significant pulls on the gluon PDF at various $x$, among the newly-fitted LHC run- 1 data, the $8 \mathrm{TeV} Z p_{T}$ ATLAS data (Exp. ID $=253)$ show the strongest overall pull in the immediate vicinity of $x=0.01, S_{g, L 2}(E) \approx-(4-5)$, approaching the pull of the E866 $p p$ absolute cross section data $($ Exp. ID $=204)$ in the same neighborhood. Meanwhile, the corresponding pulls of the inclusive Tevatron (504) and CMS $8 \mathrm{TeV}$ (545) jet-production data are even larger at slightly higher $x=0.05-0.1$; at $x \approx 0.1$ the CMS $8 \mathrm{TeV}$ jet data (545) have a very strong pull of $S_{g, L 2}(E) \approx+13$ against the opposing pulls of the ATLAS (544) and CMS $7 \mathrm{TeV}$ (542) jet data datasets. At even higher $x$, the best-fit behavior of the gluon PDF reflects the tradeoffs among the pulls from multiple experiments, as explicated in the caption of Fig. 26. At $x<0.01$, we notice visible competition between the inclusive (160) and charm-production (147) datasets from HERA, with constraints from other experiments being less prominent in this region. The totality of observations based on Fig. 26 is consistent with our findings based on the LM scans appearing in Sec. VA 2, as typified by Fig. 21 (left panel), wherein we identified the same experiments as imposing the most stringent constraints upon $g\left(x=0.01, Q=m_{H}\right)$.

We conclude this section by presenting Fig. 27 with the ranking plots of $L_{1}$ sensitivities computed by the PDFSENSE code according to the approach in Ref. [20]. In that article, we presented tables that rank the experiments in the CT14 $4_{\text {HERAII }}$ NNLO analysis either according to their total sensitivity to the PDFs, $f\left(x_{i}, Q_{i}\right)$, computed as 


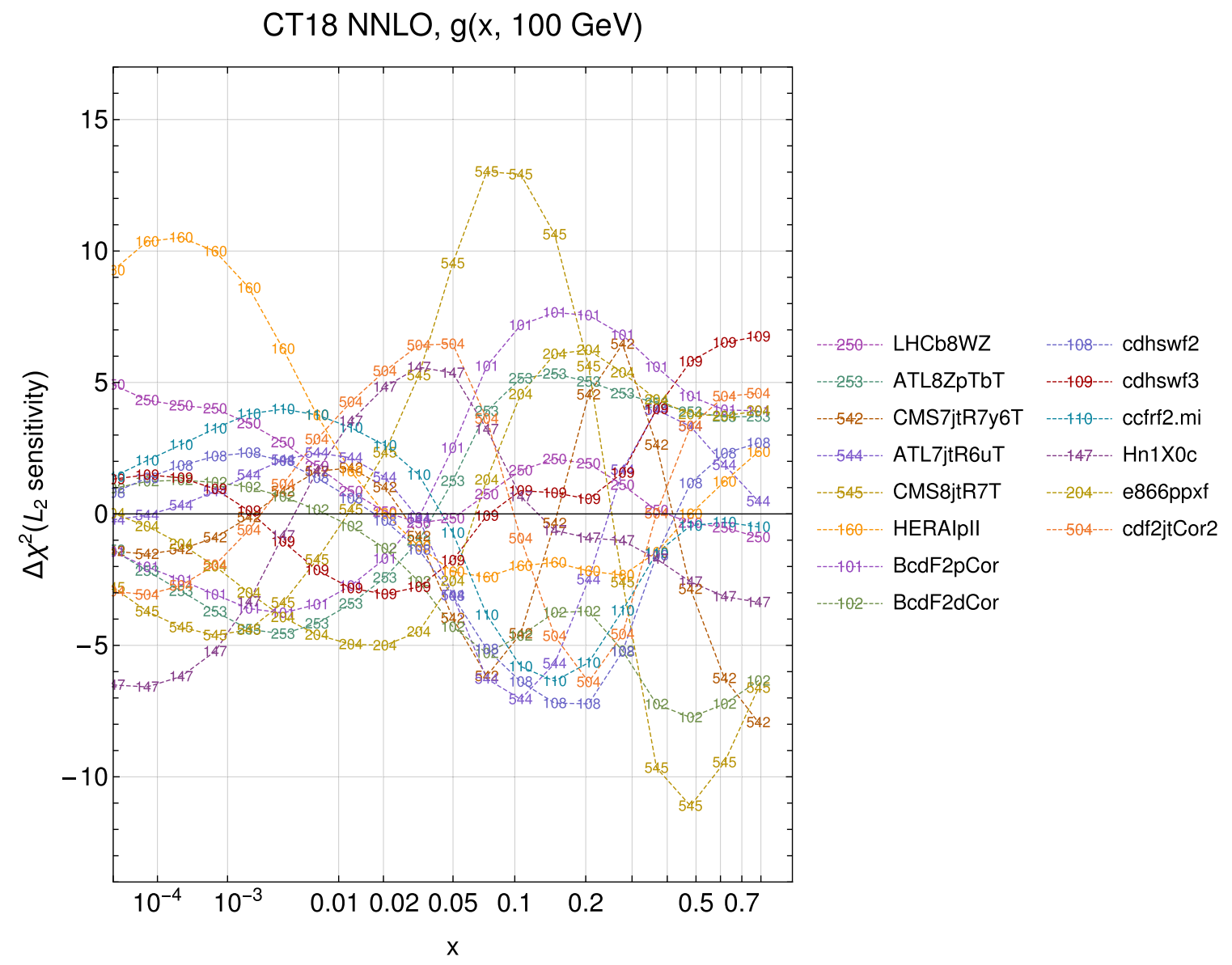

FIG. 26. The $x$-dependent $L_{2}$ sensitivity of the CT18 datasets with strongest pull upon the gluon PDF, $g(x, Q=100$ GeV). A number of tensions among the leading datasets are revealed by examining those regions of $x$ where $S_{f, L 2}(E)$ peaks for certain experiments in the "positive direction" while $S_{f, L 2}(E)$ is sharply negative for others. For instance, at $x=0.4$, the sensitivity curves indicate a strong competition of the CMS $8 \mathrm{TeV}$ jet data (Exp. ID = 545) and the BCDMS $F_{2}^{d}$ data (Exp. ID = 102), both preferring a larger $g(0.4,100 \mathrm{GeV})$, against the combined downward pull on the gluon by the BCDMS $F_{2}^{p}$ (101), CDHSW $F_{3}$ (109), E866 $p p$ Drell-Yan (204), and high- $p_{T} Z$ boson production (253) datasets. At $x \approx 0.1$, the CMS $8 \mathrm{TeV}$ jet data (Exp. ID $\left.=545\right)$ strongly pulls against the ATLAS (544) and CMS $7 \mathrm{TeV}$ (542) jet production, as well as measurements by CDHSW (108), CCFR (110), and BCDMS (102) of the DIS structure function $F_{2}(x, Q)$ on various targets.

$$
S_{f, L 1}^{\mathrm{tot}}(E) \equiv \sum_{i=1}^{N_{p t, E}}\left|S_{f, L 1}(i)\right|,
$$

or according to the average sensitivity per data point,

$$
S_{f, L 1}^{\mathrm{ave}}(E) \equiv S_{f, L 1}^{\mathrm{tot}}(E) / N_{p t, E} .
$$

These quantities respectively estimate either the total sensitivity of the experiment $E$ to the PDF, $f\left(x_{i}, Q_{i}\right)$, at the typical $\left(x_{i}, Q_{i}\right)$ probed by data points $i=1, \ldots, N_{p t, E}$, and summed over all $N_{p t, E}$ points, or the averaged sensitivity for a single data point in this experiment. The two sensitivities allow informative side-by-side comparison of the strengths of constraints from individual experiments, once again estimated in the Hessian approximation.

In Fig. 27, we present a graphical visualization of the ranking tables from Ref. [20], now recomputed for the
CT18 NNLO fit, and, for the most part, leading to similar conclusions as obtained for CT14 HERAII NNLO. The upper and lower panels of Fig. 27 correspond to the pointaveraged and total sensitivities, respectively, as discussed above. On the right are placed palettes relating the colors to the magnitudes of $S_{f, L 1}(E)$. The cells that vary from yellow to orange to red indicate experiments (listed on the left) with increasingly strong sensitivities to the PDFs, $f(x, \mu)$, given at the bottom. White or grey cells indicate experiments with minimal sensitivity to $f(x, \mu)$.

We observe that, while the HERA I + II, BCDMS, and NMC datasets have relatively low per-point sensitivity as seen in the upper panel, when aggregated over their large number of points, the experiments have very large total sensitivities to all PDF flavors seen in the lower inset. The specialized fixed-target measurements, such as CCFR, NuTeV, E605, and E866, are most sensitive to certain flavors, such as $s, \bar{u}$, and $\bar{d}$, as expected. 

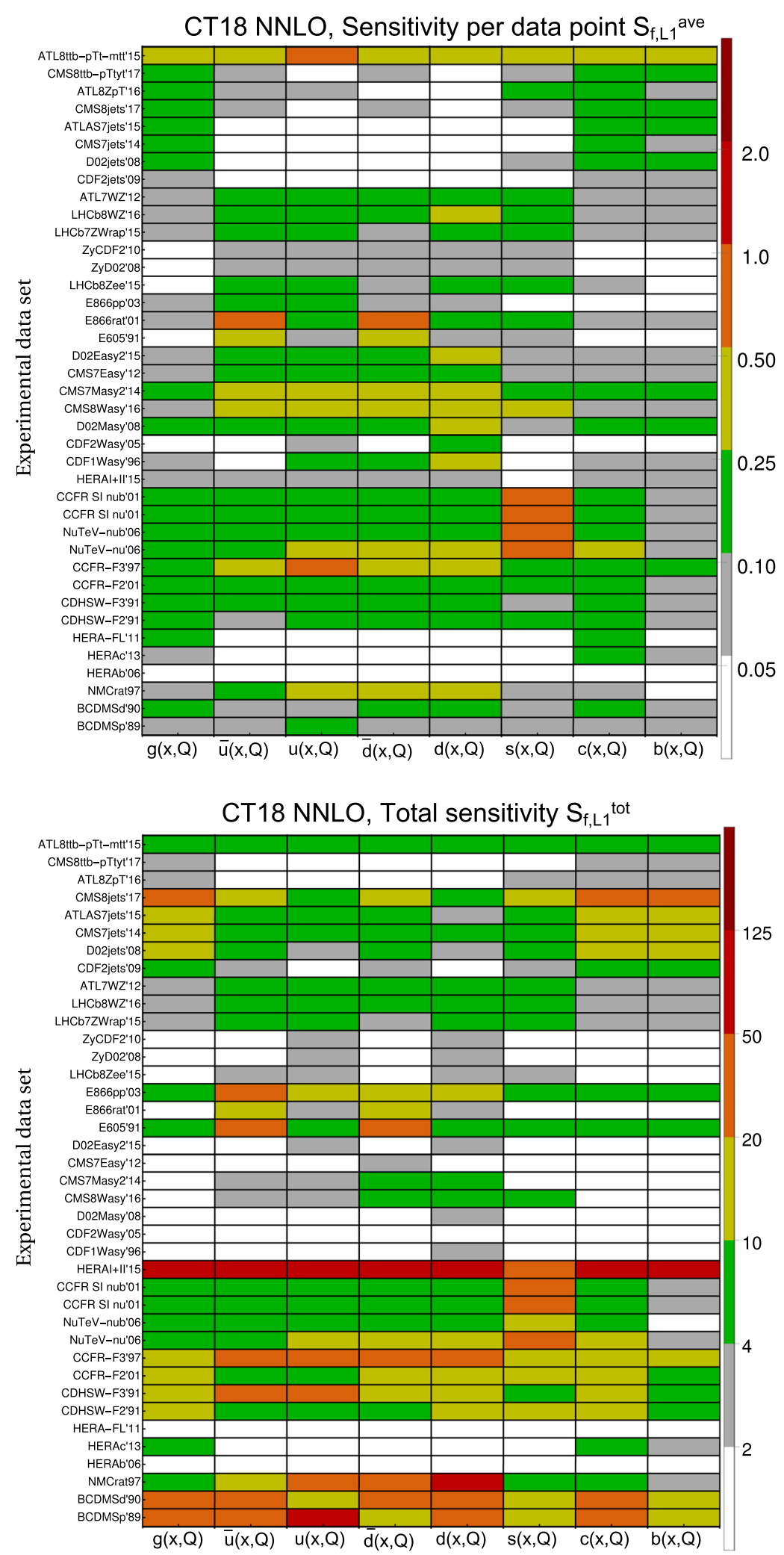

FIG. 27. $L_{1}$ sensitivities of experimental datasets to PDF flavors in the CT18 NNLO analysis, computed according to the methodology in Ref. [20]. The color of the cells in the upper (lower) inset, chosen according to the palettes on the right, indicates the point-average (cumulative) sensitivity of the experimental set on the vertical axis to the PDF flavor on the horizontal axis. 

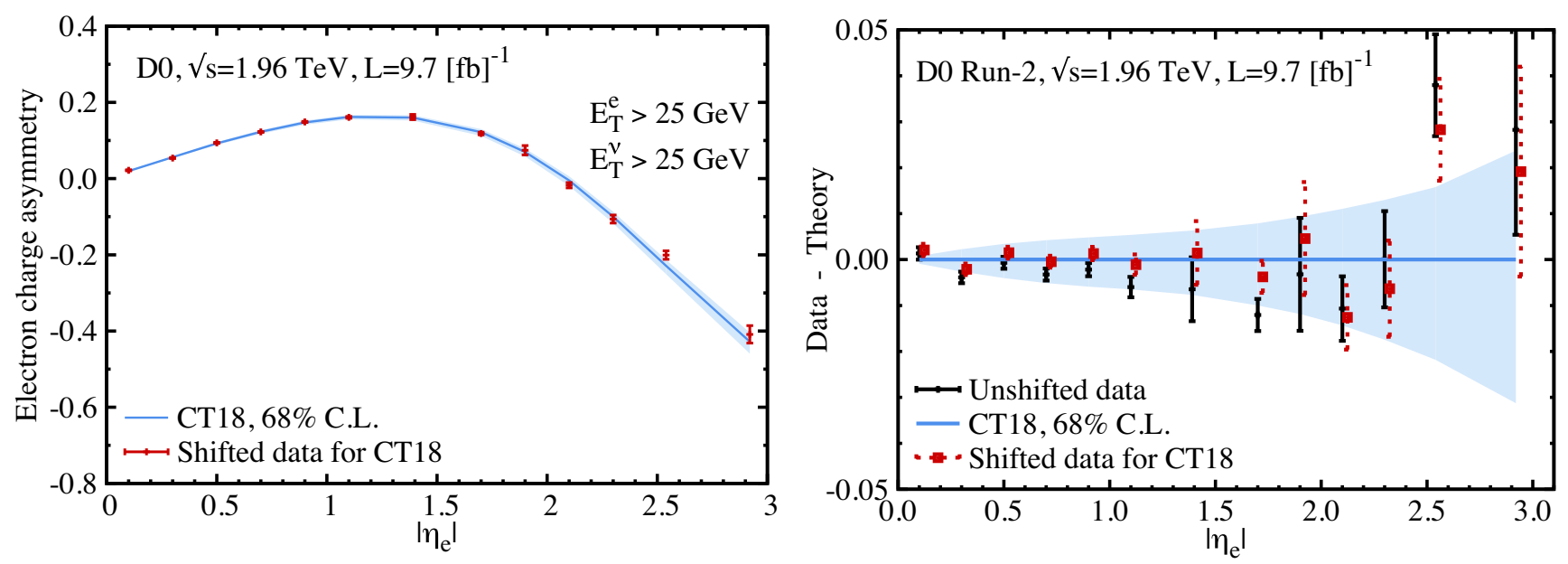

FIG. 28. A comparison of the CT18 theory with the D0 run II electron charge-asymmetry data (Exp. ID = 281). Since the asymmetry crosses zero in the shown range, the right panel shows the difference, Data-Theory, rather than the ratio, Data/Theory, as done elsewhere in this section.

Several LHC experiments, on the other hand, have strong per-point sensitivities, especially $t \bar{t}$ and high- $p_{T} Z$ production, as well as CMS $W$-charge asymmetries at 7 and $8 \mathrm{TeV}$ (see the upper inset). The total sensitivities of these experiments in the lower inset are still quite low because of their small numbers of data points $\left(N_{p t, E} \approx 10-20\right)$. On the other hand, the inclusive jet production datasets by ATLAS and $\mathrm{CMS}$ at $7 \mathrm{TeV}$, and especially by CMS at $8 \mathrm{TeV}$, despite their modest sensitivities per data point, show the highest total sensitivities among all LHC experiments because of their large numbers of data points and extended kinematic coverage.

In aggregate, while the bulk of the sensitivity in the CT18 fit still arises from HERA and fixed-target data, the LHC experiments could already reduce some PDF uncertainties, given their sizable per-point sensitivities. These uncertainty reductions have not yet been fully realized in part due to the tensions among some LHC experiments expounded upon earlier in the paper.

\section{B. Description of datasets fitted in CT18}

In this subsection, we illustrate the ability of CT18 to describe the individual experiments included in this analysis, with particular attention paid to the newly included LHC run-1 data. We organize this discussion according to the specific physical process.

\section{Vector boson production data}

\section{a. Tevatron charge asymmetry}

CT18 PDFs show a good overall agreement with the vector boson production data from fixed-target and Tevatron experiments. In particular, the high-luminosity charge asymmetry dataset 281 from D0 run-2 [78], used in our analysis since CT14 [1] and sensitive to $d(x) / u(x)$ at $x>0.1$, is well described. ${ }^{5}$

Figure 28 shows a data vs theory comparison for the electron charge asymmetry as a function of the absolute value of the electron pseudorapidity. Shifted data are represented by red points, while unshifted data are black. The absolute charge asymmetry is illustrated in the left inset of Fig. 28, while in the right one we show the DataTheory difference, where the error bars represent the total uncorrelated uncertainty (the quadrature sum of uncorrelated statistical and uncorrelated systematic errors) for both the shifted and unshifted data, as we show consistently throughout this paper, unless specified otherwise. The differences of the shifted data from theory, relative to the error bars, exemplify the goodness of fit, while the movements between the shifted and unshifted data show the effect of the correlated nuisance parameters. The theoretical predictions are computed using the code RESBOS at approximate NNLO + NNLL in QCD. The blue band represents the CT18 PDF uncertainty evaluated using the Hessian symmetric errors at the $68 \%$ C.L. We see that the data are described well by the CT18 predictions, with the exception of one high pseudorapidity bin $\left(\left|\eta_{e}\right| \sim 2.6\right)$, in which we observe a mild disagreement.

\section{b. LHC data: $L H C b$.}

As discussed previously, Drell-Yan cross-section measurements from the LHCb collaboration (Exp. IDs $=250$,

\footnotetext{
${ }^{5}$ According to the $L_{2}$ sensitivity [167], the NMC DIS data 104 and the charge asymmetry dataset 281 prefer to have a softer $d(x) / u(x)$ at large $x$ by about (15) 5 units of $\chi_{E}^{2}$, compared to the full data, in contrast to the LHCb $7 \mathrm{TeV} \mathrm{W}$ rapidity (245) and E866 $p p$ Drell-Yan (204) datasets that prefer a harder $d / u$ in the same $x$ region.
} 

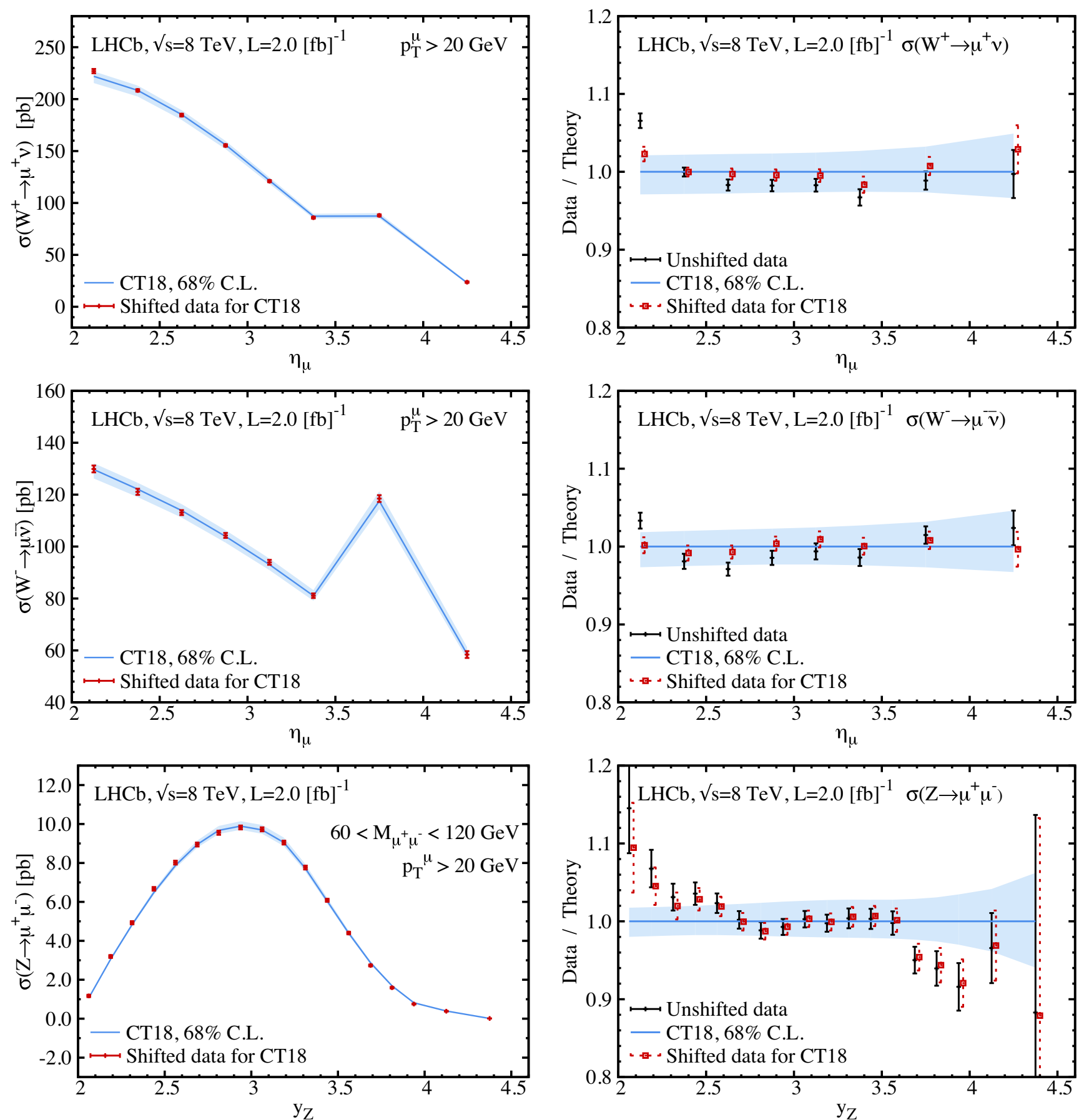

FIG. 29. A comparison of the CT18 theoretical predictions to the $W^{+}$(top), $W^{-}$(middle), and $Z^{0}$ (bottom) cross section measurements by $\mathrm{LHCb}$ at $8 \mathrm{TeV}$ in the muon decay channel $($ Exp. $\mathrm{ID}=250)$. The data are presented as cross sections for each bin, $\sigma=\frac{d \sigma}{d \eta_{\mu}} \Delta \eta_{\mu}, \frac{d \sigma}{d y_{Z}} \Delta y_{Z}$, rather than as differential cross sections. The bump in the histogram bin $3.5<\eta_{\mu}<4.0$ of $W^{-}$plot thus results from its larger bin width. A similar bump occurs in the plots for the LHCb $7 \mathrm{TeV} W / Z$ data (Exp. ID =245).

245 , and 246, in that order of importance) produce the strongest impact on CT18 PDFs among the newly introduced LHC Drell-Yan datasets. Similarly to the D0 electron charge asymmetry, in Fig. 29, the CT18 NNLO theory prediction is compared to both the shifted and the unshifted data of $W / Z$ production in the muon channel (Exp. ID $=250$ ) at $8 \mathrm{TeV}$. The analogous comparisons to $W / Z$ production in the $\mu$ channel (Exp. $\mathrm{ID}=245)$ at $7 \mathrm{TeV}$, and to $Z$ production in the $e$ channel $($ Exp. ID $=246)$ at $8 \mathrm{TeV}$ are respectively shown in Figs. 30 and 31. The NNLO theory is obtained using APPLGRID files generated with NLO MCFM, and multiplied by point-by-point $K$ factors computed with FEWZ and MCFM-8.0. 

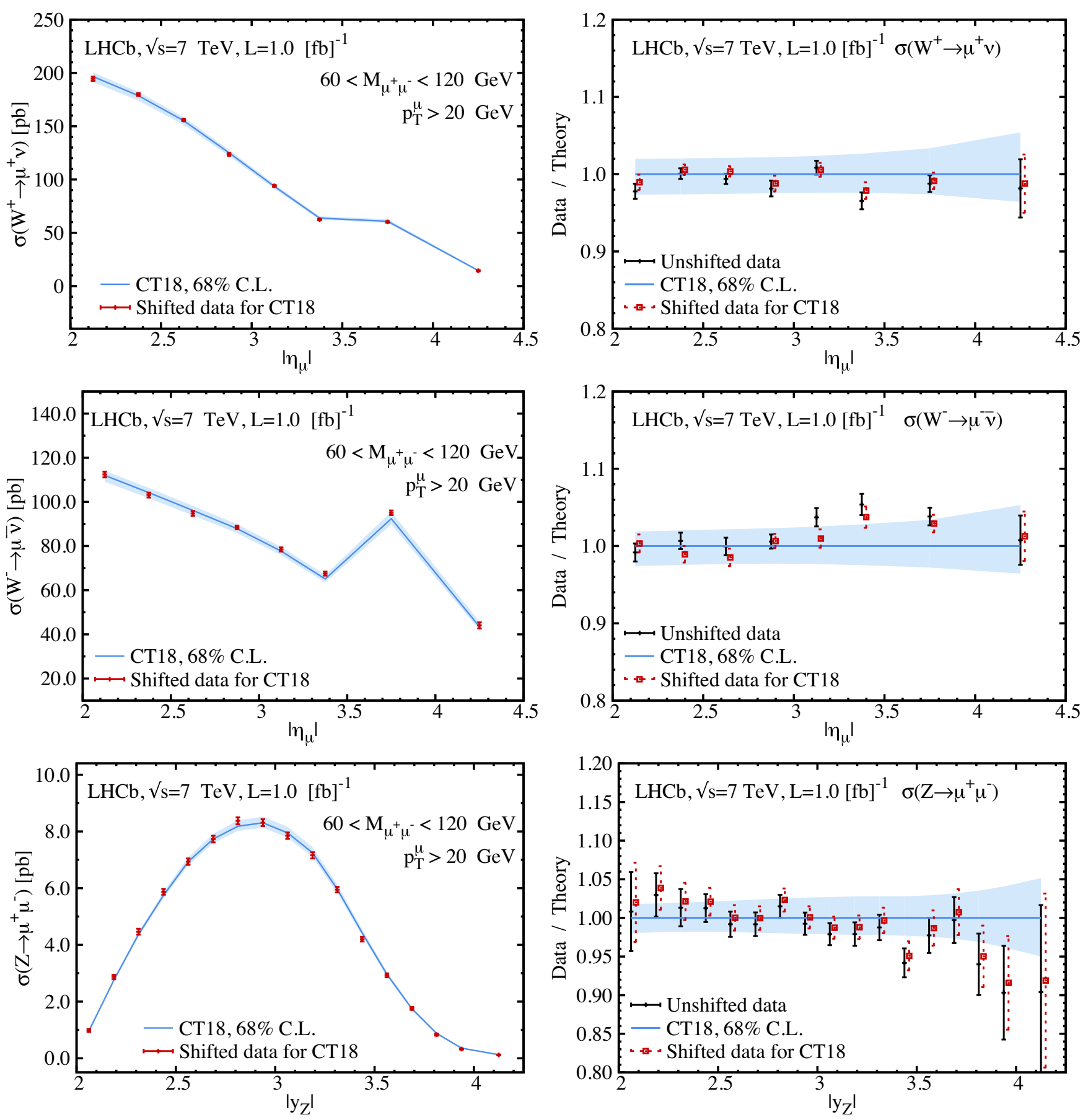

FIG. 30. Same as Fig. 29, for the LHCb $7 \mathrm{TeV}($ Exp. ID = 245).

In the case of $Z / W$ boson production at $8 \mathrm{TeV}$ in Fig. 29, theory and data agree well except for the data points near rapidity of 2 . In $Z$ boson production in all three datasets (bottom rows), some disagreement between theory and data in shape at $y_{Z}<2.5$ and $y_{Z}=3-4$ remains in spite of systematic shifts. It leads to the elevated $\chi_{E}^{2}$ for experiments 245 and 250 quoted in Table II, the discrepancy that is partially alleviated in the CT18Z fit after including the ATLAS $7 \mathrm{TeV} W / Z$ production dataset $($ Exp. ID $=248)$.
At low rapidity in $Z$ production, there is a large modeling uncertainty for the kinematic acceptance of the observed leptons. On the other hand, the discrepancy at $y_{Z} \approx 4$ shows tension with pulls from other datasets included in the global fit, such as the CMS and D0 $W$ lepton-charge asymmetry data. This tension has been investigated using the EPUMP program. In particular, we compared updated fits in which we either had included, or had not included, the first (low rapidity) bin of the $Z$-boson distribution for experiment 

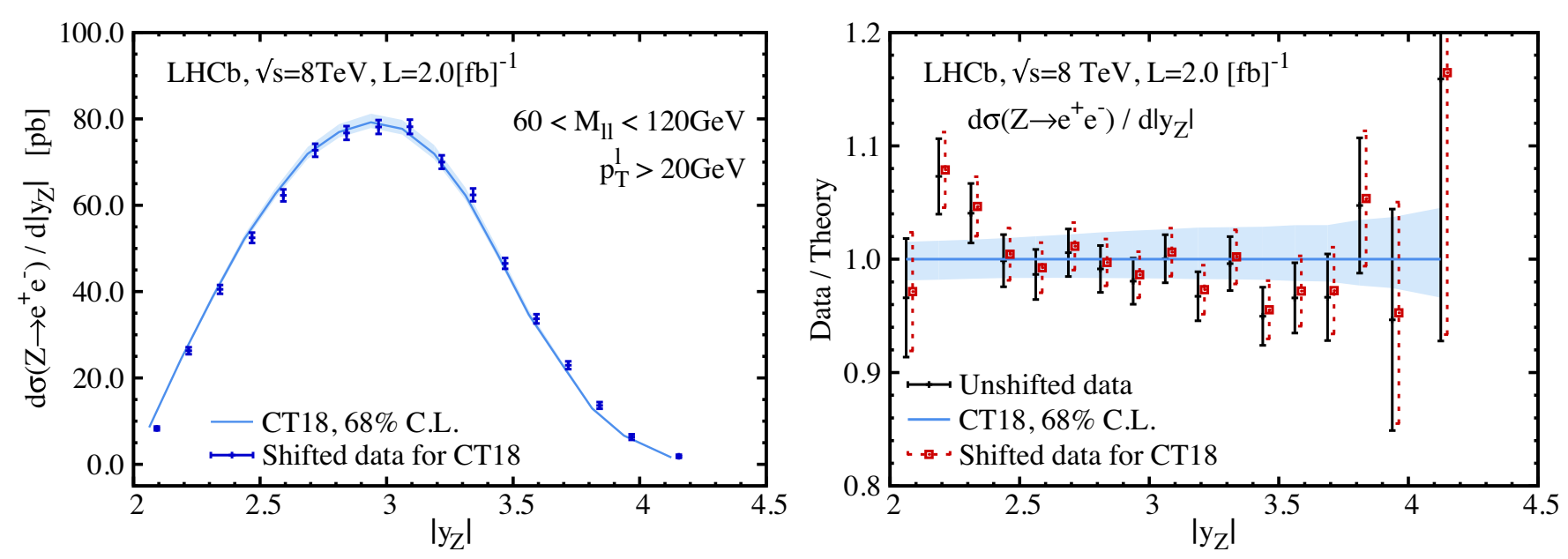

FIG. 31. A comparison of the CT18 theoretical predictions to the $Z$ rapidity distribution in $Z \rightarrow e^{+} e^{-}$production by LHCb at 8 TeV $($ Exp. ID = 246).

246. This choice had little impact on the resulting PDFs, though the $\chi_{E}^{2} / N_{p t, E}$ of the LHCb data had noticeably improved after dropping the first rapidity bin.

The quality of the CT18 fit to the individual data points can be quantified by the histograms of the shifted residuals shown in Fig. 32. When the fit to experiment $E$ is good, the histograms of its shifted residuals $r_{i}=\left(D_{i}^{s h}-T_{i}\right) / s_{i}$ and optimized nuisance parameters $\bar{\lambda}_{\alpha}$ are consistent with the standard normal distribution. For example, the third panel illustrates the distribution of $r_{i}$ for the $\mathrm{LHCb} 8 \mathrm{TeV} W^{ \pm}$and $Z$ data $($ Exp. ID $=250)$. It indicates that there are a few data points with large values in the Exp. ID $=250$ dataset. As expected, the large residuals result from the first few rapidity bins near $y=2$ in the $W^{ \pm}$and $Z$ data, and from $3.5 \lesssim y_{Z} \lesssim 4$ between 3.5 and 4 in the $Z$ data. Another useful criterion is the examination of the distribution of nuisance parameters needed to fit the Exp. ID $=250$ data, which is shown in the left panel of Fig. 33. The distribution of nuisance parameters deviates from the normal distribution, with two nuisance parameters having particularly large values $(-3$ and +3.7$)$. The right panel of Fig. 33 represents the $L_{2}$ sensitivity of these data to various PDF flavors at $Q=100 \mathrm{GeV}$. We see that the LHCb data prefers lower $u, \bar{u}$ PDFs at $x<10^{-2}$, as compared to the full global data, somewhat higher $s$ at $x<10^{-2}$, and a higher $\bar{d}$ at $x \approx 0.2$. The plots of $L_{2}$ sensitivities for the other experiments and PDF combinations can be viewed at Ref. [167].

\section{c. LHC data: CMS and ATLAS.}

Measurements of lepton charge asymmetry at $8 \mathrm{TeV}$ (Exp. ID = 249) from the CMS collaboration are included in all the CT18 global fits. The theoretical predictions, compared with the shifted and unshifted data, are shown in Fig. 34. We see that all the experimental data are fitted well within the $68 \%$ C.L. PDF uncertainty.

In the CT18(Z) analysis, we have also included the transverse momentum $\left(p_{T}\right)$ distributions of lepton pairs produced in $Z$ decays at ATLAS at $\sqrt{s}=8 \mathrm{TeV}$ (Exp. ID $=253$ ). The theoretical predictions for these data are obtained based on the NNLO fixed-order calculations for $Z+$ jet production. We stress that we have imposed a kinematic cut $45<p_{T}^{Z}<150 \mathrm{GeV}$ to remove the low- and high- $p_{T}$ regions where this fixed-order calculation lacks the necessary accuracy. The low- $p_{T}$ data are dropped because of the missing resummation effects in our fixed-order
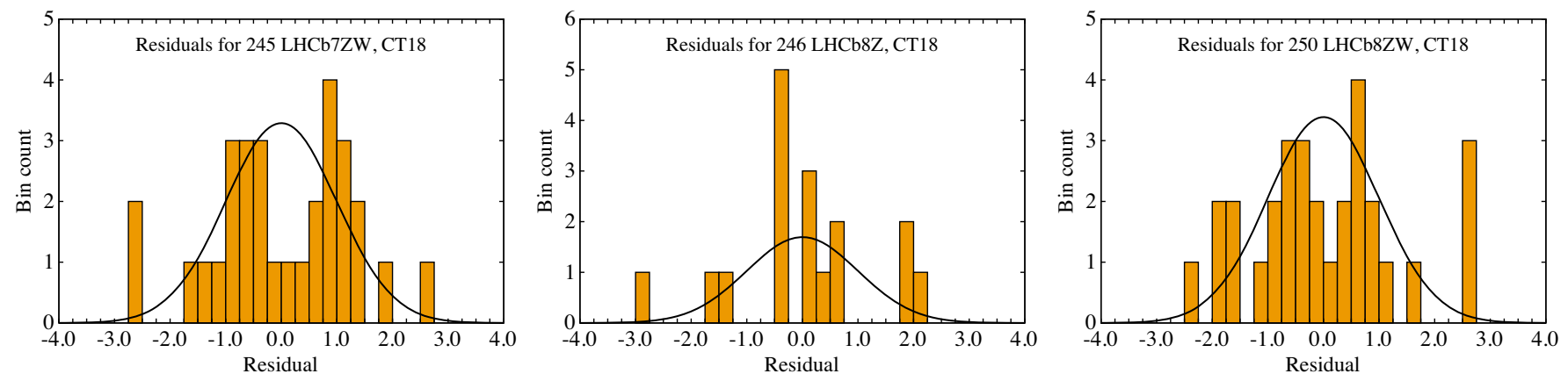

FIG. 32. Distributions of the residuals for the LHCb $W / Z$ production cross sections at 7 and 8 TeV: Exp. ID = 245 (left), 246 (center), and 250 (right). 


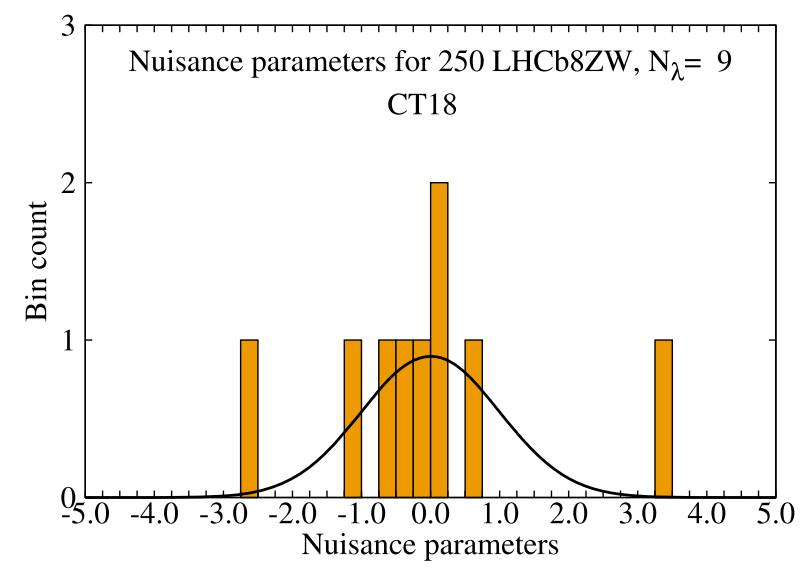

CT18 NNLO, LHCb8WZ (250), Q=100 GeV

FIG. 33. Left: distribution of nuisance parameters for the $\mathrm{LHCb} 8 \mathrm{TeV} W / Z$ cross sections (Exp. ID $=250$ ). Right: the pulls of these data on the CT18 NNLO PDFs at $Q=100 \mathrm{GeV}$, computed in terms of the $L_{2}$ sensitivity of Eq. (21) [167].
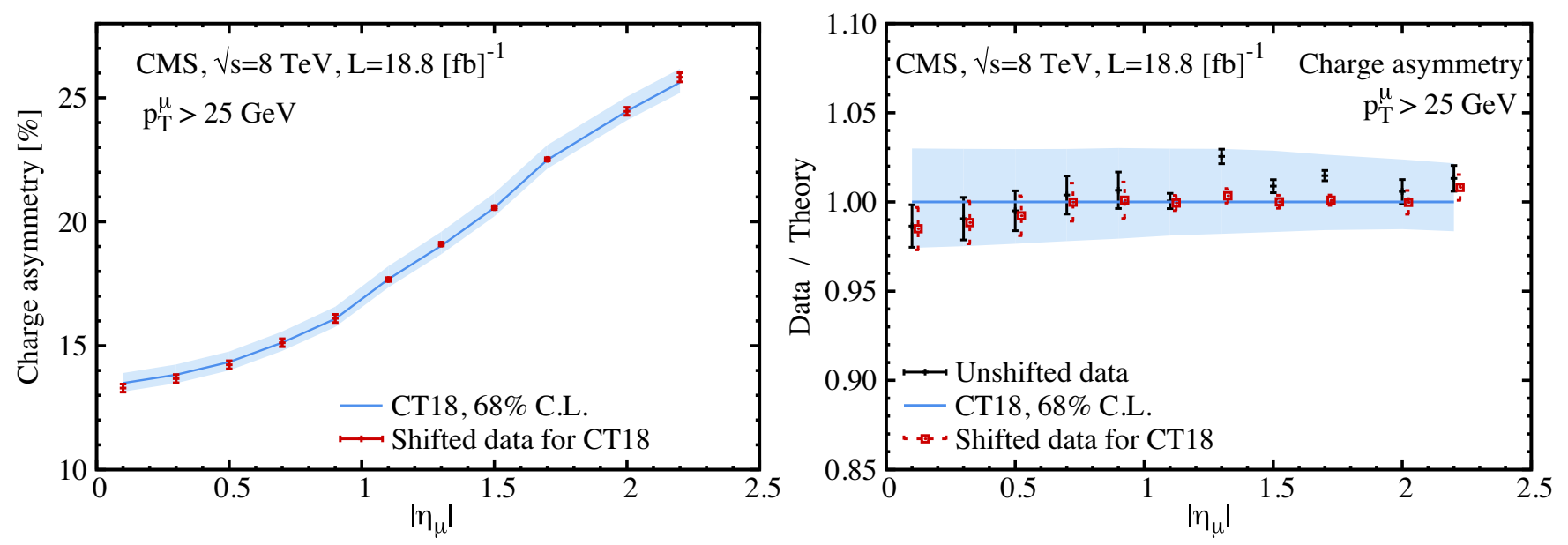

FIG. 34. A comparison of the CT18 theoretical predictions to the CMS $8 \mathrm{TeV}$ charge asymmetry data (Exp. ID $=249)$.

calculation. The high- $p_{T}$ data are dropped because (1) the constraining power of the data is small given the relatively large statistical errors, and (2) the EW corrections are nonnegligible, as will be discussed in Sec. V C.

As a practical implementation, we generated in-house NLO APPLGRID files with MCFM and multiplied them by the NNLO/NLO $K$ factors computed as the ratios of the NNLO and NLO cross sections published in Refs. [52,53,168-171]. To account for non-negligible fluctuations in the NNLO theoretical prediction, we have included an additional $0.5 \%$ theoretical Monte Carlo uncertainty, estimated by the standard deviation for a smooth curve fitted to discrete $K$ factors. The nominal renormalization and factorization scales are chosen as

$$
\mu_{R}=\mu_{F}=M_{T, \ell \bar{\ell}}=\sqrt{\left(p_{T, \ell \bar{\ell}}\right)^{2}+M_{\ell \bar{\ell}}^{2}}
$$

assuming the unity prefactor in the scales. We have also investigated the QCD scale dependence by multiplying the renormalization and factorization scales independently by the scaling factors of 2 and $1 / 2$. Specifically, the scale uncertainty is estimated using the envelope of the sevenpoint scale variation:

$$
\begin{aligned}
\left(\mu_{R}, \mu_{F}\right)= & {[(1 / 2,1 / 2),(1,1 / 2),(1 / 2,1),(1,1),} \\
& (1,2),(2,1),(2,2)] \times M_{T, \ell \bar{\ell}}
\end{aligned}
$$

All these combinations of QCD scales describe the shape of the ATLAS $Z p_{T}$ data fairly, however, the data prefer higher-than-nominal normalizations, which can be accommodated either by increasing the overall normalization of theory by 1-2 standard deviations of the luminosity uncertainty, reducing $\mu_{R}$, or by increasing $\alpha_{s}$ to 0.120 0.124 [172]. As a result, we get a marginally better $\chi^{2}$ for $\mathrm{ID}=253$ using the scales

$$
\mu_{R}=M_{T, \ell \bar{\ell}} / 2, \quad \mu_{F}=M_{T, \ell \bar{\ell}},
$$

with the negligible difference in the PDFs compared to the other scales. The CT18 NNLO theoretical predictions with the scales as in Eq. (29) are compared to the ATLAS $8 \mathrm{TeV}$ 

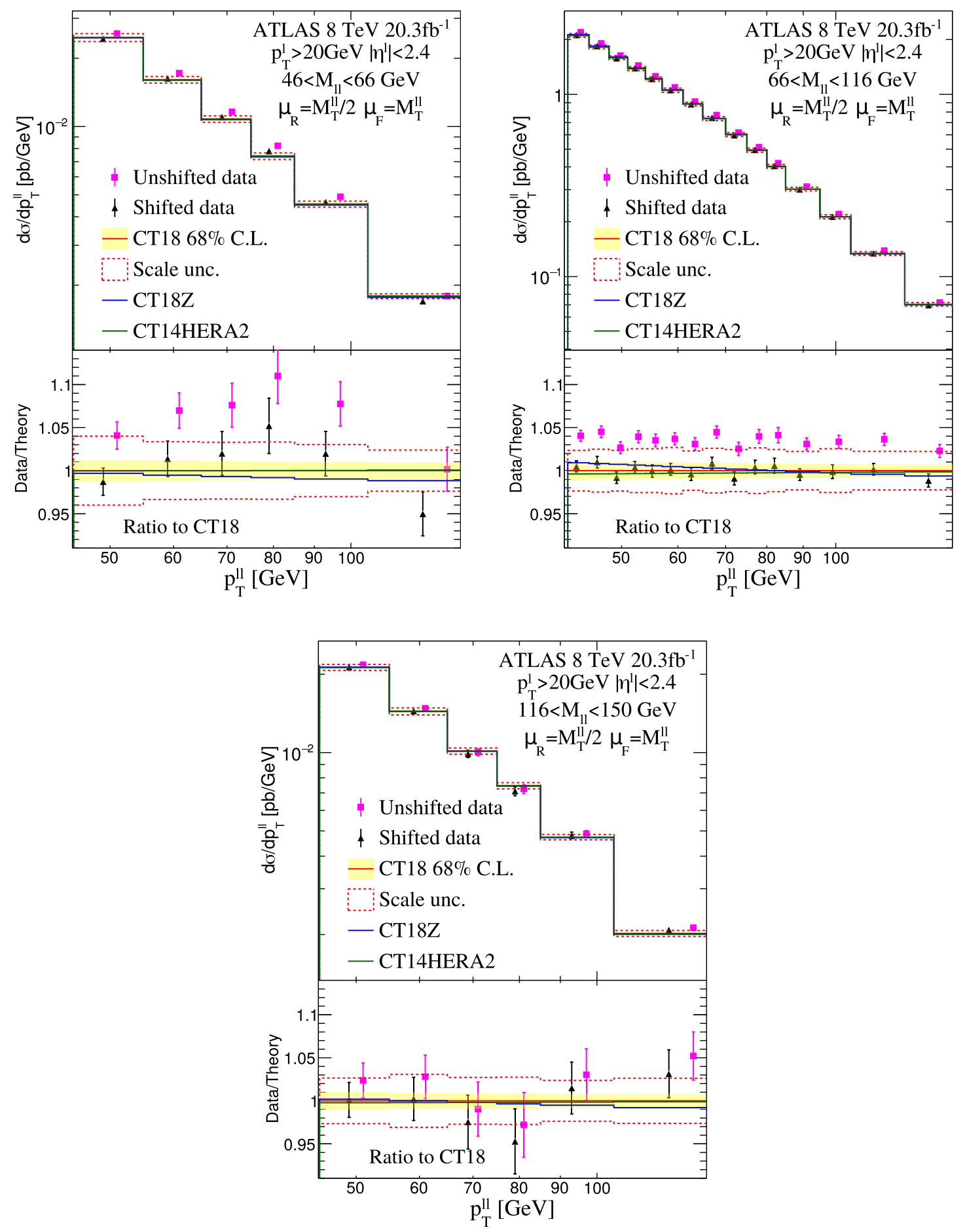

FIG. 35. Theoretical predictions for lepton pair transverse momentum distribution, $p_{T, \ell \bar{\ell}}$ based on the CT14 ${ }_{\text {HERAII }}$ CT18, and CT18Z NNLO PDFs, using QCD scales $\mu_{R}=M_{T, \ell \bar{\ell}} / 2, \mu_{F}=M_{T, \ell \bar{\ell}}$ and compared with the ATLAS 8 TeV measurements. The yellow band represents the PDF uncertainty calculated with the symmetric Hessian method at the $68 \%$ C.L. The dashed band represents the scale uncertainty.

data in Fig. 35. We obtain $\chi_{E}^{2} / N_{p t, E} \sim 1$ and fairly describe all three invariant-mass bins after allowing for an upward shift by $1.2 \sigma$ in the overall normalization. The complementary figure with the scales as in Eq. (27), also having $\chi_{E}^{2} / N_{p t, E} \approx 1$, cf. Table II, and requiring a shift in the overall normalization by $2 \sigma$, is included in Supplemental Material [27].

In Fig. 36, the distributions of the residuals (in the left subfigure) and nuisance parameters (in the right subfigure) of these data are shown. We see excellent agreement of 

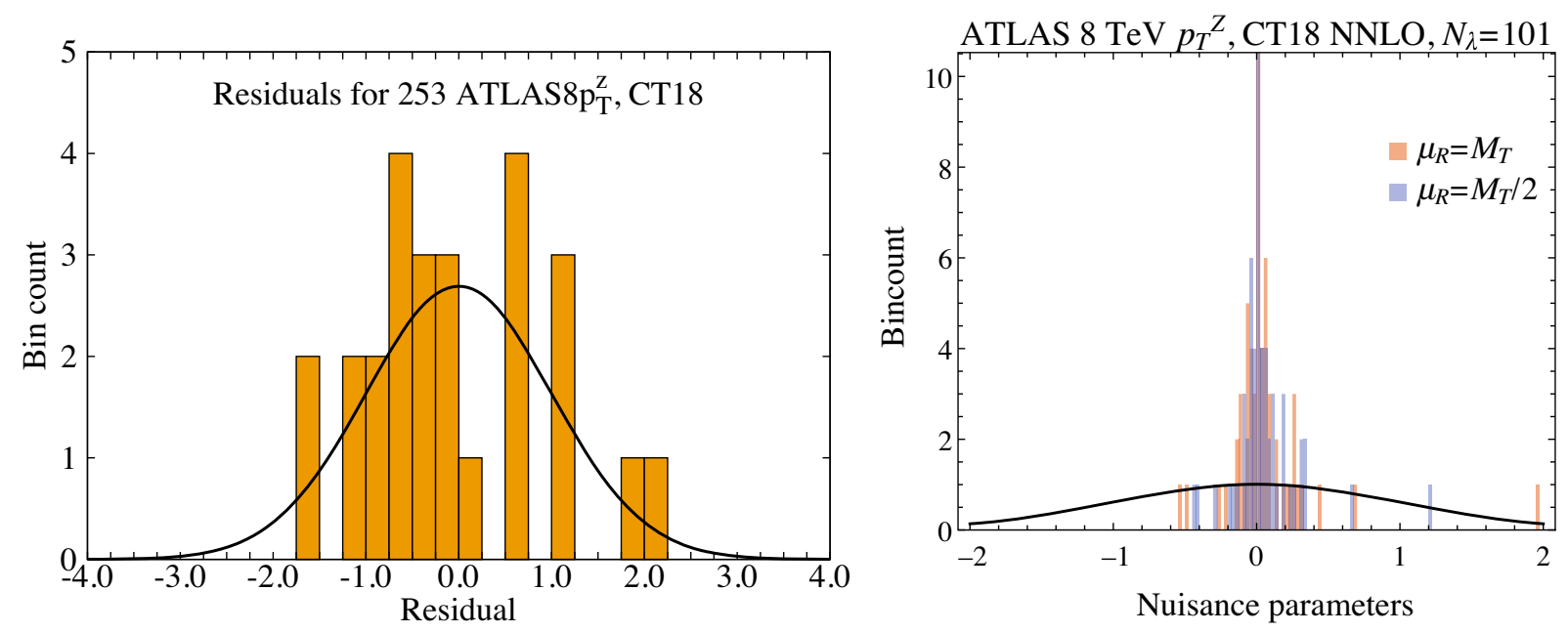

FIG. 36. Left: Distribution of the $\chi^{2}$ residuals for the ATLAS $8 \mathrm{TeV} Z p_{T}$ data (Exp. ID $=253$ ) obtained using the nominal QCD scales in Eq. (27). Right: distributions of the respective nuisance parameters obtained for $\mu_{R}=M_{T, \ell \bar{\ell}}$ (default) and $M_{T, \ell \bar{\ell}} / 2$, with $\mu_{F}=M_{T, \ell \bar{\ell}}$ in both cases.

theory and data in the distribution of residuals. In the distribution of nuisance parameters, out of 101 nuisance parameters in this process, only one parameter, associated with the overall normalization, is increased by more than $1 \sigma(2 \sigma)$ for $\mu_{R}=M_{T, \ell \bar{\ell}} / 2\left(M_{T, \ell \bar{\ell}}\right)$. More than 70 nuisance parameters are too close to zero in these fits, perhaps indicating that the experiment has tabulated too many evanescent systematic effects. (Such excess of very small nuisance parameters is not uncommon for the LHC experiments, as discussed at the beginning of Sec. V and in Sec. IV.E of [37].)

The alternative scales $\mu_{F, R}=M_{\ell \bar{\ell}}$ have been also tried and resulted in a worse description of the shape of the $p_{T}$ distribution (not only the normalization) and elevated $\chi^{2}$.

The remaining difference cannot be explained by the EW corrections, since the EW corrections are small and negative (see Sec. V C), pulling the theory further away from the data. Instead, the systematic shift in the normalization can possibly be ascribed to the missing higher-order $\left(\mathrm{N}^{3} \mathrm{LO}\right)$ corrections, implied by two observations. First, the NNLO corrections to the $Z p_{T}$ are generally as large as $10 \%$, which indicates slow convergence of the perturbative expansion. Second, the large scale uncertainty (about $3-4 \%$ ) is also an indication that the missing higher-order effects may be significant.

\section{Jet data}

Historically, inclusive jet production has played an important role in constraining the gluon density, $g(x, Q)$, as evidenced by the impact that the older jet data from the Tevatron run-II had on the CT10 and CT14 global analyses. CT18 now also implements inclusive jet production data at even higher collider energies and luminosities, measured by the ATLAS and CMS collaborations at the LHC, as described in Sec. II B 4.

\section{a. Tevatron run-II data}

First, we examine the fits to the Tevatron run-II jet data. The CDF run-II data shown in Fig. 37 is not perfectly described by NNLO theory (has an elevated $\chi_{E}^{2} / N_{p t, E} \approx 1.7$ according to Table I) and prefers a somewhat different shape of the gluon PDF $g(x, Q)$, compared to the average of all experiments, according to the $L_{2}$ sensitivity plot for $g(x, Q)$ in Fig. 26. The D0 run-II jet data, depicted in Fig. 38, show better agreement with the rest of the datasets.

\section{b. Run-1 LHC data}

The CT18 fit can describe the LHC CMS and ATLAS jet data, depicted in Figs. 39-41, after the decorrelation of some correlated systematic errors, as laid out in Sec. II B 4 and Appendix E, as well as the inclusion of a $0.5 \%$ overall uncorrelated systematic error for all the LHC jet data, as discussed in Sec. II B 4.

Although the agreement with theory in the CT18 analysis is reasonable, we note some tensions among the LHC inclusive jet datasets themselves, especially the CMS results at 7 (Exp. ID = 542) and $8 \mathrm{TeV}($ Exp. ID = 545). These tensions are particularly pronounced for some parton flavors in the specific kinematic regions-most evidently, for the gluon PDF, as quantified by the LM scans and $L_{2}$ sensitivity profiles plotted in Figs. 21 and 26, respectively. For the ATLAS inclusive jet data at $7 \mathrm{TeV}$, the best fit requires the correlated errors to shift the raw data downward in the smaller rapidity regions, but to shift the raw data upward at high rapidities. The majority of optimal nuisance parameters $\lambda_{\alpha}$ for the CT18 NNLO PDF set, shown in the histograms included in Supplemental Material [27], are distributed narrowly about $\left|\lambda_{\alpha}\right| \sim 0$. For the CMS $8 \mathrm{TeV}$ dataset, four nuisance parameters out of 28 require 


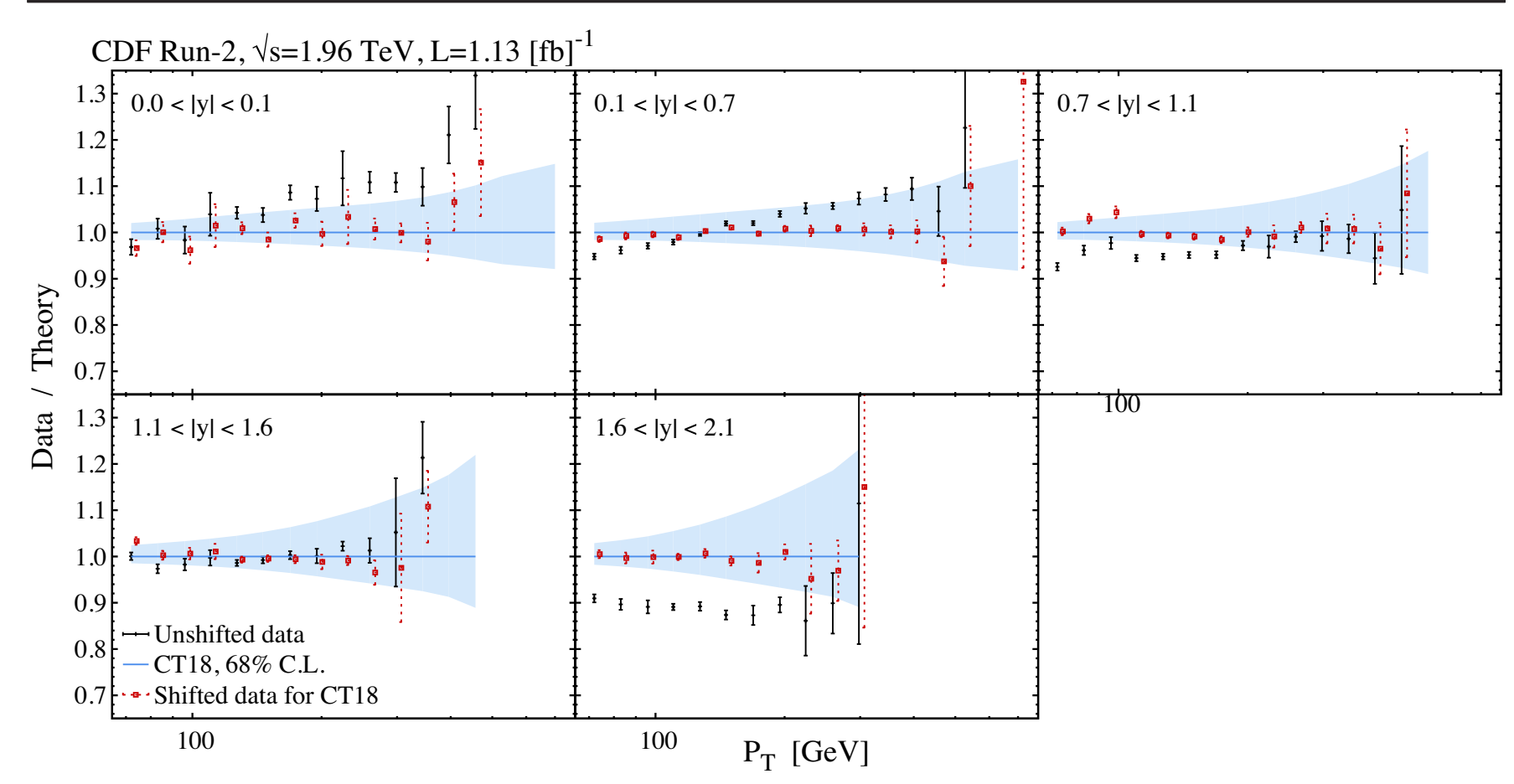

FIG. 37. Data/Theory values for CT18 NNLO and CDF run-2 jet data (Exp. ID = 504).

absolute correlated shifts larger than two-a larger count than is expected based on the assumed normal statistics.

\section{Top-quark pair production data}

The two $t \bar{t}$ datasets included in the CT18 global analysis are described well, as shown by the values of $\chi^{2}$ and effective Gaussian variable $S_{E}$ given in the latter two rows of Table II. In particular, for the CMS (Exp. ID = 573) and ATLAS (Exp. ID $=580)$ datasets included in the fit, we obtain $S_{E}=0.6$ and $S_{E}=-1.1$, respectively. For a detailed point-by-point description of the $t \bar{t}$ agreement with the theory, in Figs. 42 and 43 we show plots of the (Data)/(Theory) ratio for both datasets. For simplicity,

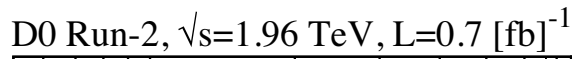

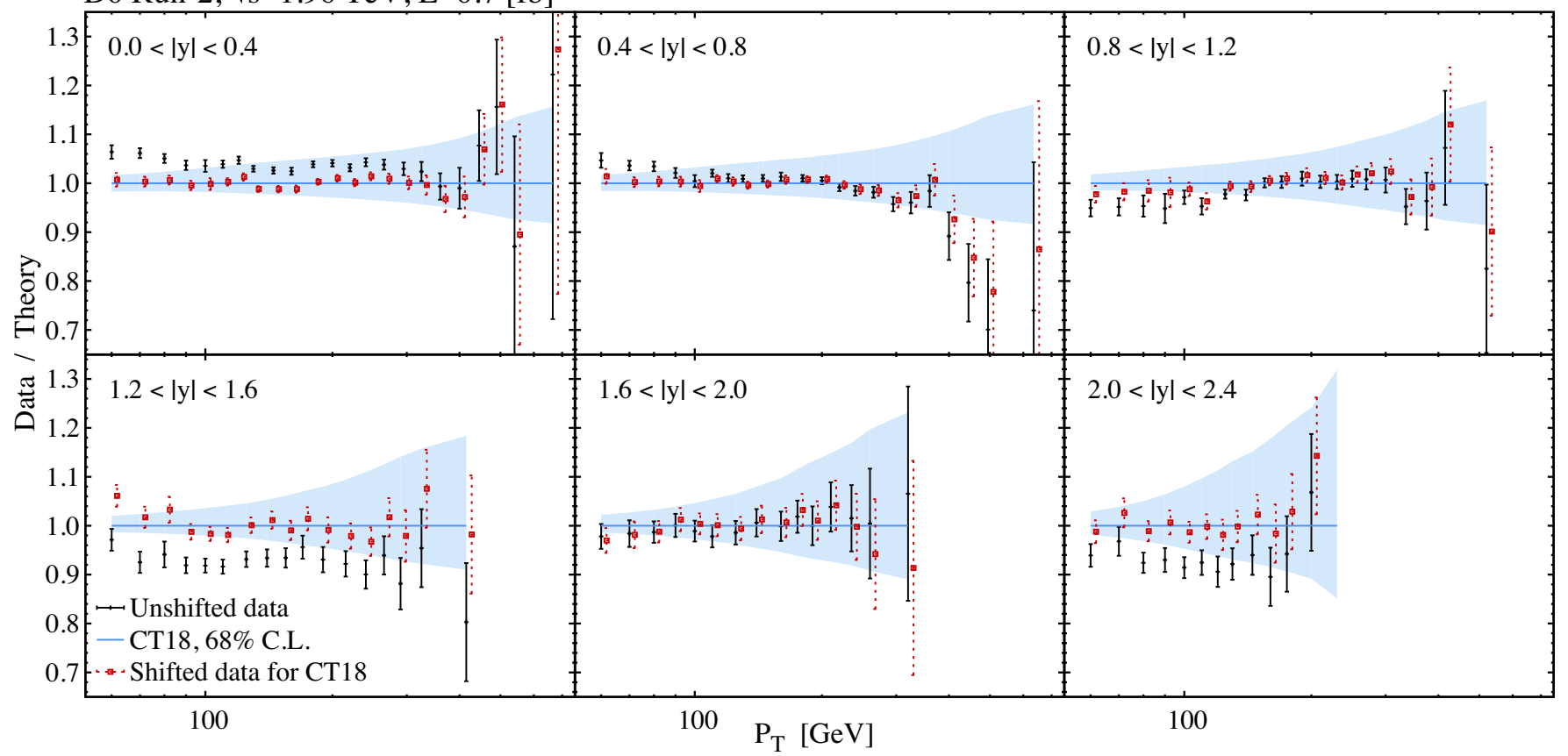

FIG. 38. Data/Theory values for CT18 NNLO and D0 run-2 inclusive jet production (Exp. ID $=514)$. 


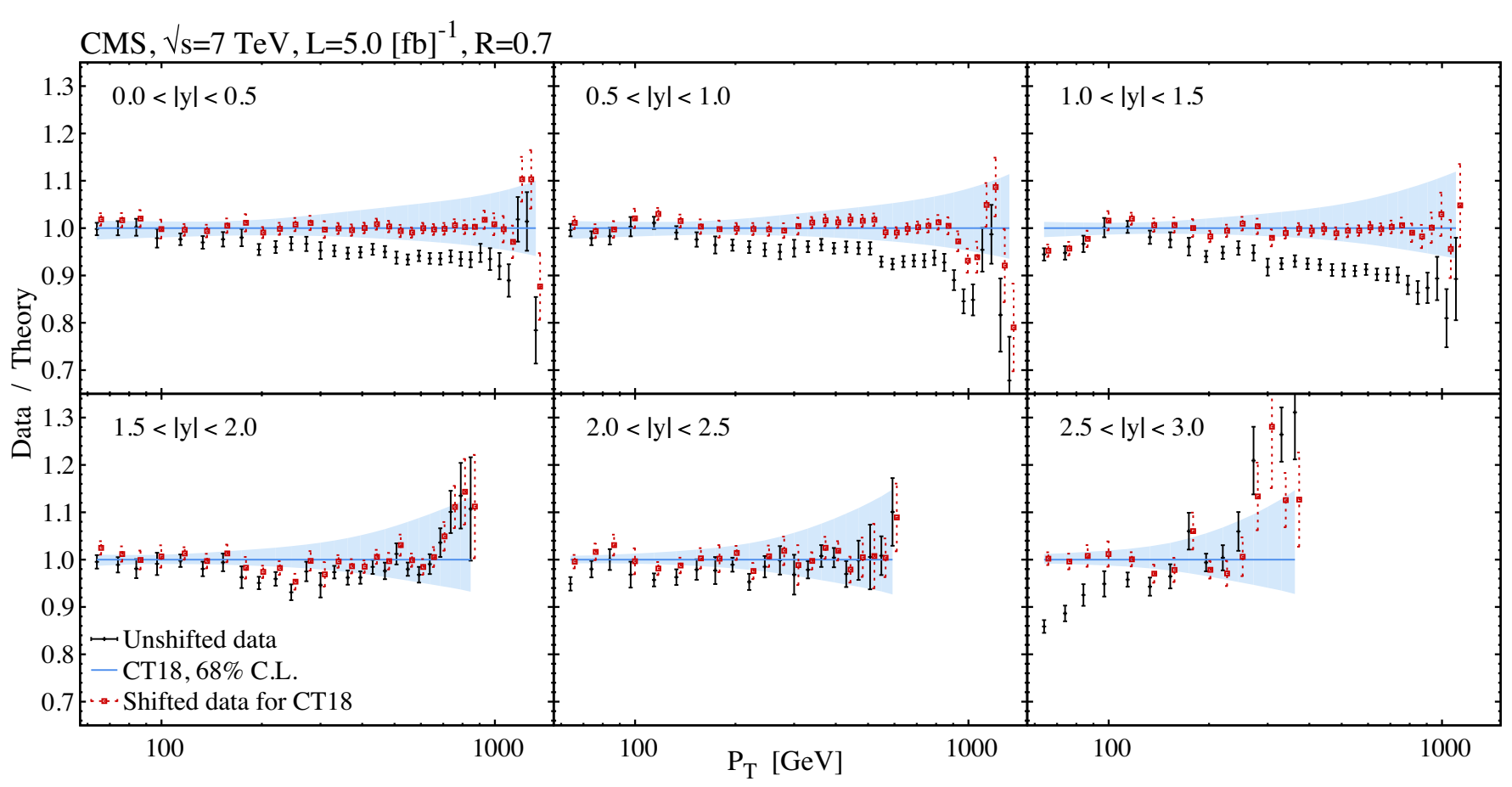

FIG. 39. Data/Theory values for CT18 NNLO and CMS $7 \mathrm{TeV}$ inclusive jet production (Exp. ID = 542).

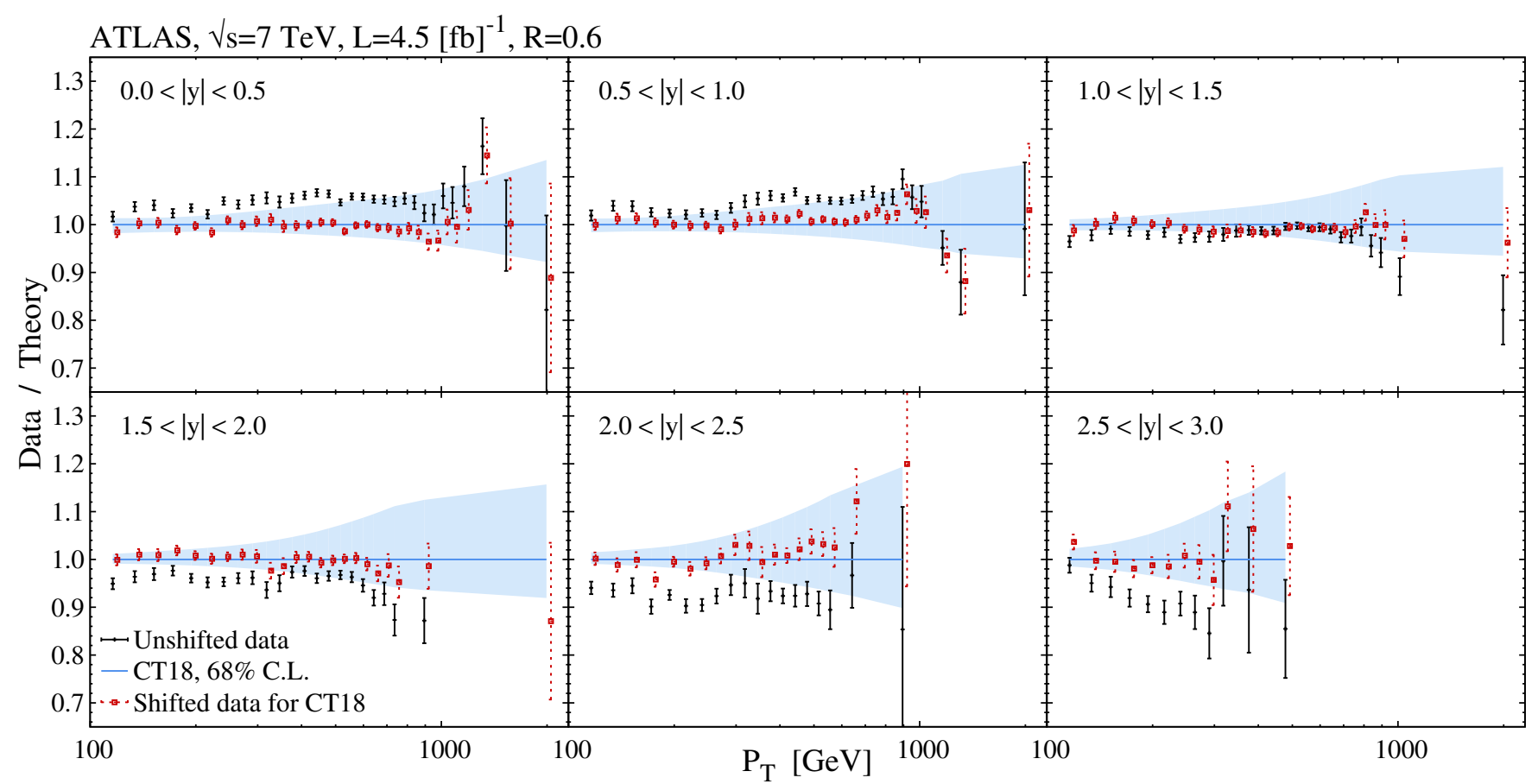

FIG. 40. Data/Theory values for CT18 NNLO and ATLAS 7 TeV inclusive jet production (Exp. ID = 544).

the error bars for the CMS data in Fig. 42 were calculated by including both statistical and correlated systematic errors listed in Tables 5 and 7 of Ref. [88].

The statistical correlations for these measurements (Table 6 in Ref. [88]) are not included in the CT18 analysis because of technical difficulties in the realization of the nuisance parameter representation for this statistical correlation information. The nuisance parameter representation is the one and only default representation utilized in all CT analyses. The statistical correlations released by the CMS collaboration are given in terms of the covariance matrix representation. Despite this, we independently cross-checked the impact of the statistical correlations by using the EPUMP software which is able to process the 


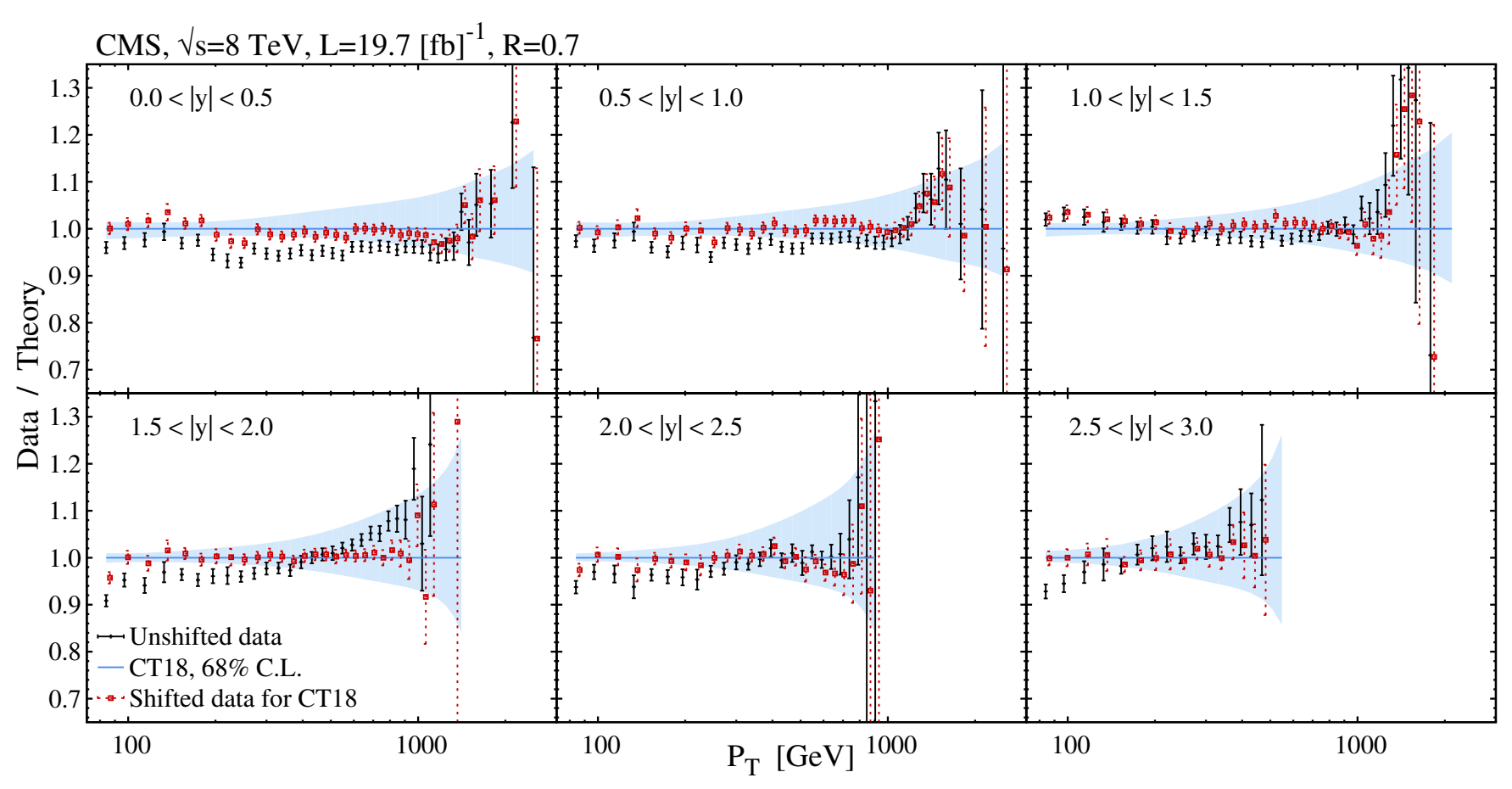

FIG. 41. Data/Theory values for CT18 NNLO and CMS $8 \mathrm{TeV}$ inclusive jet production (Exp. ID = 545).

correlation information when given in terms of covariance matrix. The conclusion is that the inclusion of statistical correlations has negligible impact on the resulting EPUMP updated PDFs. The CT18 baseline $\chi^{2}$ value obtained for the CMS (Exp. ID = 573) data with the inclusion of the statistical correlation covariance matrix increases by about 5 units, as compared to the value (18.9) given in Table II. (See Refs. [173] and [100] for related discussions on the inclusion of these measurements in global PDF analyses.)

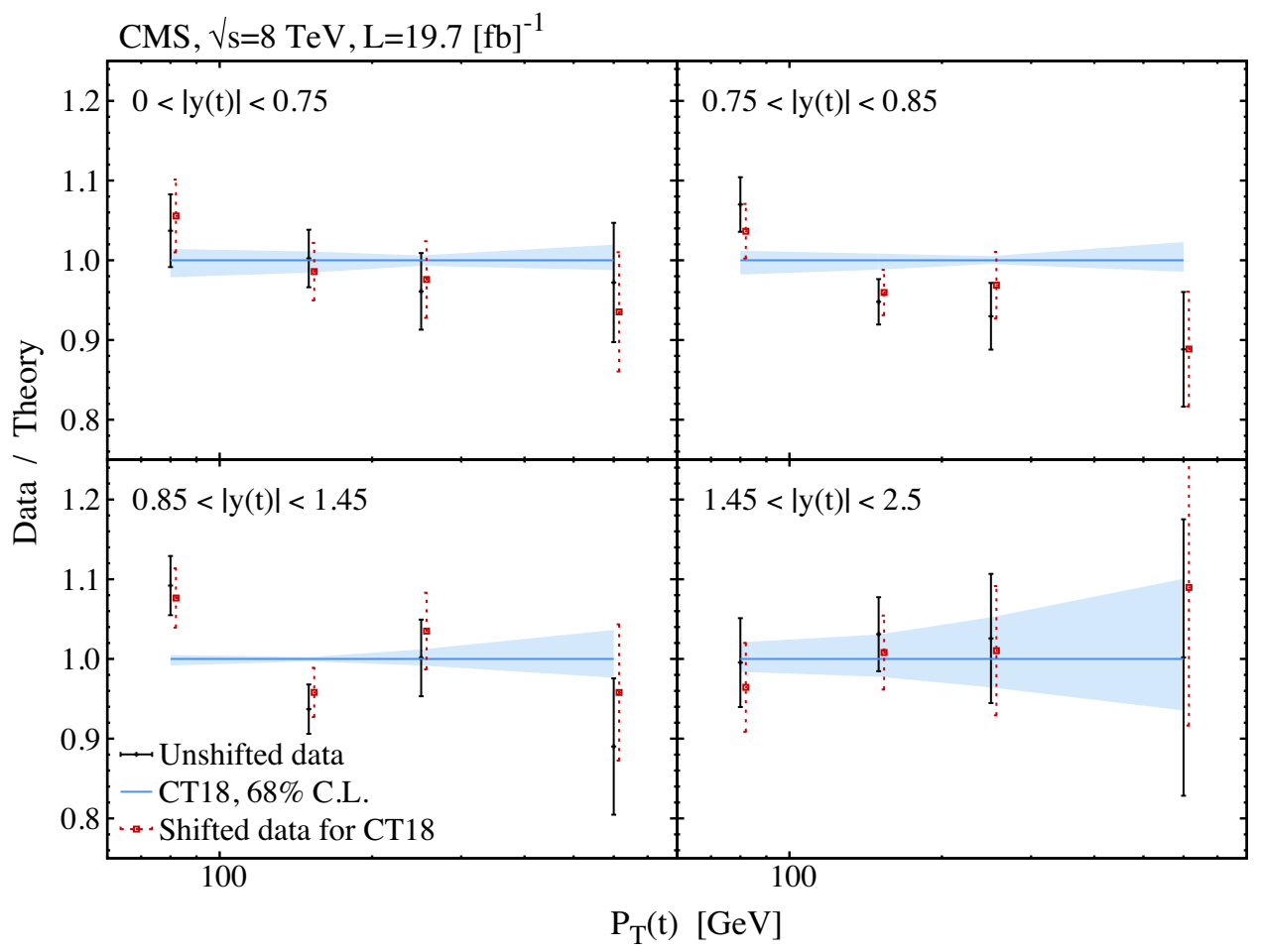

FIG. 42. (Data)/(Theory) comparison for the CMS $8 \mathrm{TeV} t \bar{t}$ production data $($ Exp. ID $=573$ ) as a function of the transverse momentum of the top (anti)quark. 

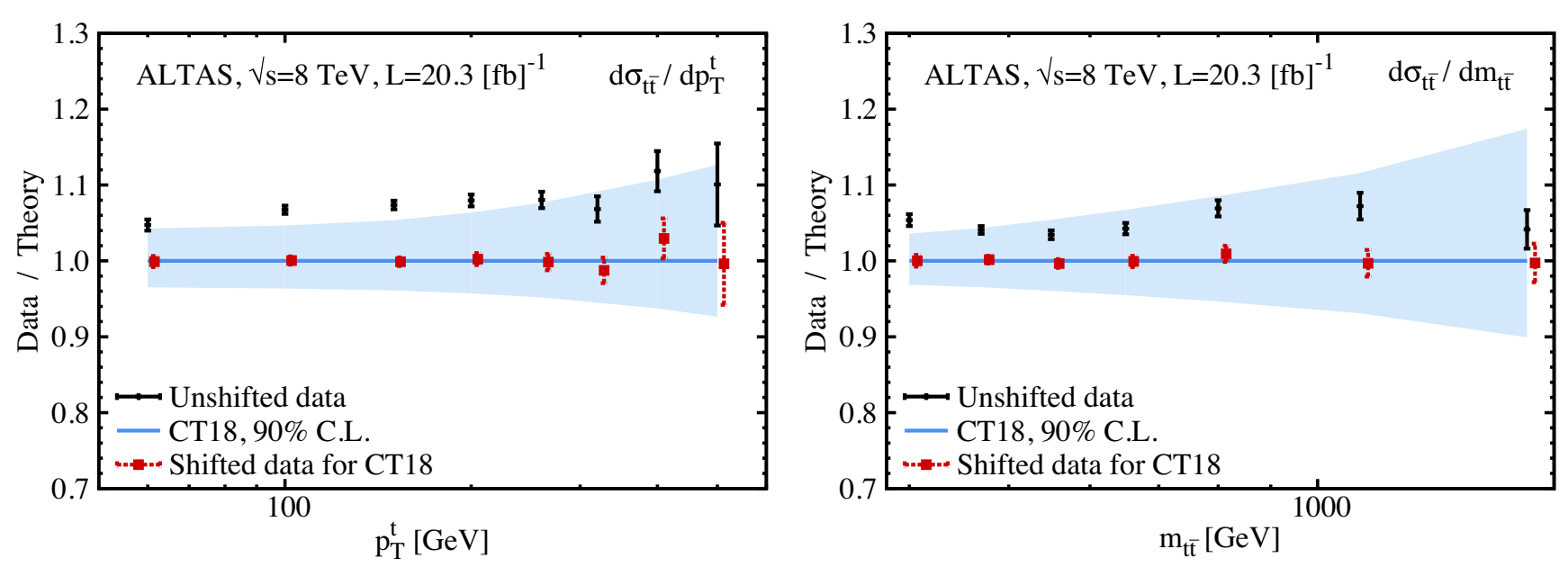

FIG. 43. (Data)/(Theory) comparison for the ATLAS $8 \mathrm{TeV} t \bar{t}$ production data $($ Exp. ID $=580)$ as a function of the $t \bar{t}$ transverse momentum (left) and invariant mass (right).

In Fig. 42, the top-quark $p_{T}$ distribution at CMS is fitted reasonably well across the four rapidity bins examined here. We find modest deviations between theoretical predictions and the (un)shifted data for some points in the intermediate rapidity bins, $0.75<\left|y_{t}\right|<0.85$ and $0.85<\left|y_{t}\right|<1.45$, contributing to the somewhat broader distribution of residuals. Notably, the effect of correlated errors in fitting the CMS data is relatively minimal, given the fact that the shifted (red) and unshifted (black) data are very similar, as observed in Fig. 42. Correlated systematics are nonetheless important for some cross section values, allowing the data values to shift enough to be within $1 \sigma$ distance from the CT18 prediction.

In contrast, achieving a very good description of the analogous ATLAS $p_{T, t}$ and $m_{t \bar{t}}$ distributions shown in Fig. 43 critically depends on the use of nuisance parameters to compensate for correlated systematics, as seen in Fig. 43. The uncorrelated errors are small (less than 1-2\%) in most bins of this dataset. On the other hand, the systematic errors are sizable and the systematic shifts lead to a very good agreement between theory and data, with $\chi_{E}^{2} / N_{p t, E}=$ 9.4/15 for CT18 NNLO.

\section{Dimuon production}

Charm-quark production cross sections in neutrino deepinelastic scattering provide key low- $Q$ constraints on the strangeness PDF at $x>10^{-2}$. In the CT14 NNLO analyses, the charm-quark production cross section were calculated at NLO in QCD [174-176] in the S-ACOT- $\chi$ variable-flavornumber (VFN) scheme [118-120,177]. Recently, chargedcurrent coefficient functions in DIS have been calculated to NNLO in QCD, including quark mass dependence $[122,178]$. This calculation, in a fixed-flavor-number (FFN) scheme with three light-quark flavors, is published in the form of fast interpolation tables for the kinematics of the CCFR and NuTeV dimuon experiments $[63,64]$.
The CT18 analysis still uses an NLO theory prediction in the S-ACOT- $\chi$ VFN scheme because it matches the precision of the CCFR and $\mathrm{NuTeV}$ experimental datasets. Implementation of charm-quark mass effects at NNLO demands not only the NNLO charged-current cross section in an ACOT-like VFN scheme, which is not yet available, but also consistency in implementation of QCD radiative effects in the CCFR and NuTeV studies of their systematics. In the case of NuTeV [63], unfolding of events, acceptance estimations, ${ }^{6}$ and studies of charm fragmentation were done using LO and NLO programs, with systematic uncertainties that exceed the magnitude of the NNLO radiative contribution, as concluded in Refs. [122,178], and that depend on the charm quark mass and an (arguably small [179]) nuclear correction. In the CT analyses, the CCFR and NuTeV dimuon cross sections are implemented by assuming the $c \rightarrow \mu$ branching ratio of 0.099, as in Section 5.2.1 of [63]. The normalization uncertainty of $10 \%$ is treated as fully correlated over the $\nu$ channel and similarly over the $\bar{\nu}$ channel. The rest of systematic uncertainties are added in quadrature. Overall, the discussion in Ref. [178] indicates that, for the kinematics of CCFR and $\mathrm{NuTeV}$, the differences between the NNLO results from the FFN scheme and any VFN scheme are expected to be significantly smaller than the precision of experimental data.

As a cross check, we have carried out alternative fits, labeled CT18(Z)-charmDIS NNLO, using the NNLO FFN calculations for dimuon production cross sections. In the case of CT18-charmDIS NNLO, the global $\chi^{2}$ is reduced by 6 units (compared to CT18), with the reduction in the $\chi_{E}^{2}$ for the dimuon data of the order of 1-2 units. For CT18ZcharmDIS NNLO, the global $\chi^{2}$ and the $\chi_{E}^{2}$ for the dimuon

\footnotetext{
${ }^{6} \mathrm{CCFR}$ and NuTeV collaborations apply significant acceptance corrections for extracting the charm-quark production cross sections from dimuon cross sections. These corrections were estimated at NLO precision only.
} 

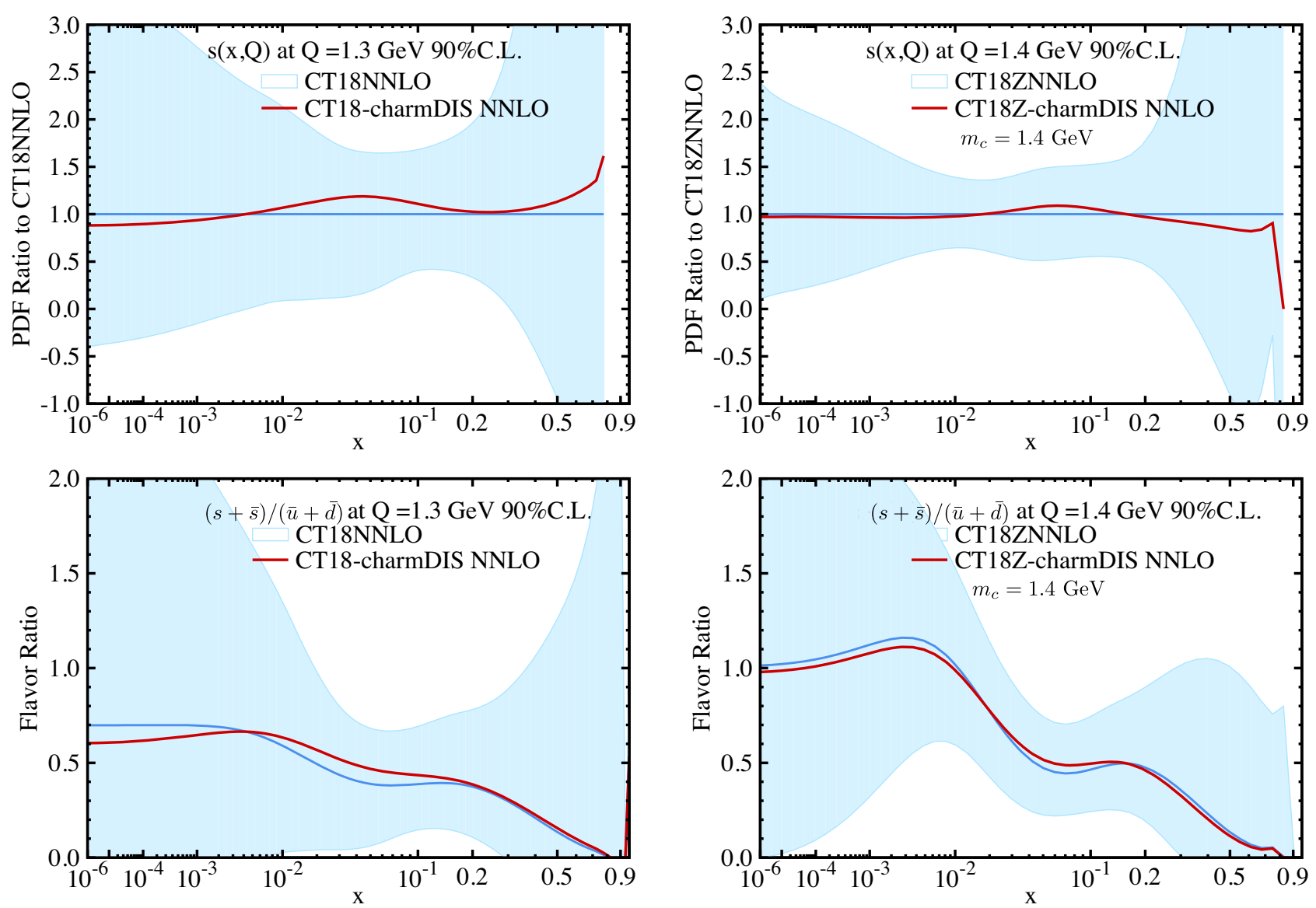

FIG. 44. Strange-quark distribution $s(x, Q)$ and ratio $R_{s}(x, Q)$ in CT18 (left) and CT18Z (right) NNLO fits, compared with alternative fits using QCD NNLO cross sections for CCFR and NuTeV measurements.

data are reduced by 11 and 8 units, respectively, compared to CT18Z. In both cases the NNLO predictions provide a marginally better agreement with the data.

The impact of these choices on the strange-quark PDF has also been cross checked. The strange-quark PDF $s(x, Q)$ and the ratio $R_{s}(x, Q)$ defined in Eq. (10) are compared in Fig. 44 for the nominal CT18(Z) fits and their "charmDIS NNLO" alternatives. In the CT18-charmDIS fit, we observe a slight increase of the strange-quark PDF at $x \approx 0.1$. This outcome is consistent with the PDF profiling results in Ref. [178] and reflects negative NNLO QCD corrections in the same $x$ region. In the CT18Z-charmDIS fit, with the ATLAS $7 \mathrm{TeV} W / Z$ data included, the PDFs change less as compared to the CT18-charmDIS fit. Furthermore, the changes due to the NNLO contribution to dimuon production are small compared to the size of the PDF uncertainties, as one can also infer from the relative stability of the $\chi^{2}$ values for the nominal and alternate fits.

The tendency of the NNLO corrections to the dimuon cross sections to slightly increase the strangeness to higher values at $x \approx 0.1$ has independently been confirmed by using the fast Hessian updating technique with EPUMP [25], as well as by the MMHT group [19], cf. Appendix A. Increasing the $c \rightarrow \mu$ branching ratio from 0.099 to 0.092 adopted by MMHT [149] only marginally increases $s(x, Q)$ in CT18 at $x>0.1$, while also slightly increasing the $\mathrm{CCFR}+\mathrm{NuTeV} \chi^{2}$ values.

Finally, to estimate the impact of the NNLO corrections to the charm-quark production cross section on the simultaneous inclusion of the ATL7ZW and dimuon datasets, we

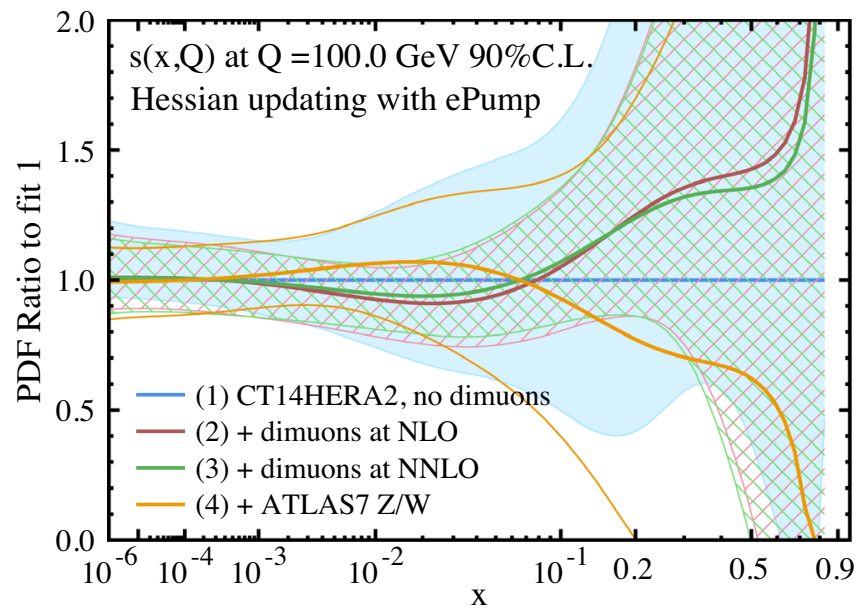

FIG. 45. Comparison of $s$ PDF at $Q=100 \mathrm{GeV}$ for various fits. See the main text for its detail. 
TABLE VIII. A summary of electroweak corrections to the LHC precision data considered for CT18(Z). For each process, we indicate the primary observable, an approximate upper bound for the EW correction, references for computing the EW corrections, and whether the data were adopted in CT18(Z) with or without EW corrections.

\begin{tabular}{|c|c|c|c|c|c|}
\hline Data & Observables & $\begin{array}{l}\text { Size of EW (and PI) } \\
\text { corrections }\end{array}$ & Ref. & $\begin{array}{l}\text { Data included } \\
\text { in the CT18(Z)? }\end{array}$ & $\begin{array}{l}\text { EW corrections } \\
\text { included in the fits }\end{array}$ \\
\hline Inclusive jet & $p_{T} \sim 1.4 \mathrm{TeV}$, central & $8 \%$ & [132] & Yes & Yes \\
\hline$t \bar{t}$ & $p_{T}^{t} \sim 500 \mathrm{GeV}$ & $-5 \%$ & [136] & Yes & No \\
\hline$W^{+}\left(W^{-}\right)$ & & $-0.4(0.3) \%$ & & & \\
\hline DY low-mass & $46<M_{\ell \bar{\ell}}<66 \mathrm{GeV}$ central & $+1.5 \%(\mathrm{PI})+6 \%(\mathrm{EW})$ & & & \\
\hline DY Z-peak & $\begin{array}{c}66<M_{\ell \bar{\ell}}<116 \mathrm{GeV} \\
\text { central (forward) }\end{array}$ & $<0.1 \%(\mathrm{PI})-0.3(-0.4 \%)(\mathrm{EW})$ & [39] & CT18Z & Yes \\
\hline DY high-mass & $\begin{array}{c}116<M_{\ell \bar{\ell}}<150 \mathrm{GeV} \\
\text { central (forward) }\end{array}$ & $+1.5 \%(\mathrm{PI})-0.5(-1.2 \%)(\mathrm{EW})$ & & & \\
\hline high-mass Drell-Yan & $M_{\ell \bar{\ell}} \sim 1 \mathrm{TeV}$ & $+5 \%(\mathrm{PI})-3 \%(\mathrm{EW})$ & FEWZ & No & $\ldots$ \\
\hline$Z p_{T}$ & $p_{T} \sim m_{Z}$ & about $-5 \%$ & {$[135]$} & Yes & No \\
\hline & $p_{T} \sim 1 \mathrm{TeV}$ & about $-30 \%$ & {$[135]$} & No & $\ldots$ \\
\hline
\end{tabular}

performed a series of NNLO fits illustrated in Fig. 45. There, we compare the strange-quark PDF obtained from four different fits that have been updated with EPUMP. PDF set (1) is the base fit obtained from the CT14 HERAII dataset by removing the $\mathrm{NuTeV}$ and CCFR dimuon data. Adding back those four dimuon datasets, with NLO and NNLO predictions, yields the sets (2) and (3), respectively. PDF set (4) is obtained by adding the ATL7ZW dataset, without the dimuon datasets. While PDF set (3) (found using the NNLO dimuon cross sections) yields an $s$ PDF that is marginally closer to that constrained by the ATL7ZW data for $10^{-3} \lesssim x \lesssim 10^{-1}$, the improvement is still too weak to resolve the tension between the ATL7ZW and dimuon datasets.

\section{Electroweak corrections}

In this subsection, we present a summary of the electroweak (EW) corrections for the LHC data which were considered, and, in some cases, applied, to the CT18(Z) fits. In general, we have not used data for which EW corrections are large, especially if the data do not provide significant constraints to the PDFs. EW corrections tend to be larger in those kinematical regions for which the statistical errors of the data are also sizable, such that those measurements which are most impacted by EW corrections are often less sensitive to the PDFs. We note that PI contributions are also important in kinematical regions afflicted by large EW corrections, but these are of opposite sign, thus leading to partial cancellation. As we do not include an explicit photon PDF in the CT18(Z) PDFs, there is a potential to over-estimate the impact of EW corrections in the kinematical regions where they are greatest. For those EW corrections described below which were applied to the $\mathrm{CT} 18(\mathrm{Z})$ fits, the implementation was via multiplicative $K$ factors.

In Table VIII, we summarize the upper bounds upon the EW corrections to data considered for CT18(Z), indicating whether these data were fitted and whether EW corrections were applied. Of these, the largest EW corrections are for the inclusive jet cross section, being as large as $8 \%$ in the highest $p_{T}$ bins of the central rapidity region. The EW corrections for $t \bar{t}$ production have already been noted in Sec. III B 4, with the largest EW corrections for the $p_{T}(t)$ distribution. At high- $p_{T}(t)$ values approaching $500 \mathrm{GeV}$, the EW correction is $-5 \%$ before decreasing rapidly at softer values of $p_{T}(t)$. For $t \bar{t}$ observables other than the $p_{T}(t)$ spectra, EW corrections are negligible compared to the experimental uncertainty. Given the experimental precision of the $8 \mathrm{TeV} t \bar{t}$ information over $p_{T}<500 \mathrm{GeV}$, we do not include EW corrections when fitting these data, but such corrections will likely be required to describe future measurements at higher $p_{T}$.

The EW corrections to the inclusive $W^{+}, W^{-}$, and $Z / \gamma^{*}$ production data have been investigated in Ref. [39] using the MCSANC framework [180]. For $W^{+}$and $W^{-}$production, the EW corrections were found to be $-0.4 \%$ and $-0.3 \%$, respectively. In the $Z$-peak region $\left(66<M_{\ell \bar{\ell}}<116 \mathrm{GeV}\right)$ for neutral-current (NC) Drell-Yan (DY) with central (forward) selections, ${ }^{7}$ the EW corrections are about $-0.3(-0.4) \%$, with only a weak kinematical dependence on the observables $M_{\ell \bar{\ell}}$ and $y_{\ell \bar{\ell}}$. We estimate that photoninduced dilepton production $\left(\gamma \gamma \rightarrow l^{+} l^{-}\right)$contributes to $Z$-peak NC DY by less than $0.1 \%$. For the low-mass $\left(46<M_{\ell \bar{\ell}}<66 \mathrm{GeV}\right)$ region, the EW corrections are $+6 \%$ independent of rapidity selection criteria, and, for high-mass $\left(116<M_{\ell \bar{\ell}}<150 \mathrm{GeV}\right) \mathrm{NC}$ DY production, the EW corrections are $-0.5 \%(-1.2 \%)$ for the central (forward) selection, with a very weak dependence on the $\eta_{l}$ and $y_{\ell \bar{\ell}}$ bins. The PI contributions are $1.5 \%$ for both $M_{\ell \bar{\ell}}$ bins. Given the small impact of the low- and high-mass DY data on the PDF fits, we decided not to include the lowmass, high-mass, and forward Z-peak DY data in the

\footnotetext{
${ }^{7}$ The central selection requires both leptons in the central region, $\left|\eta_{l}\right|<2.5$, while the forward one requires one central and one forward $\left(2.5<\left|\eta_{l}\right|<4.9\right)$ leptons.
} 


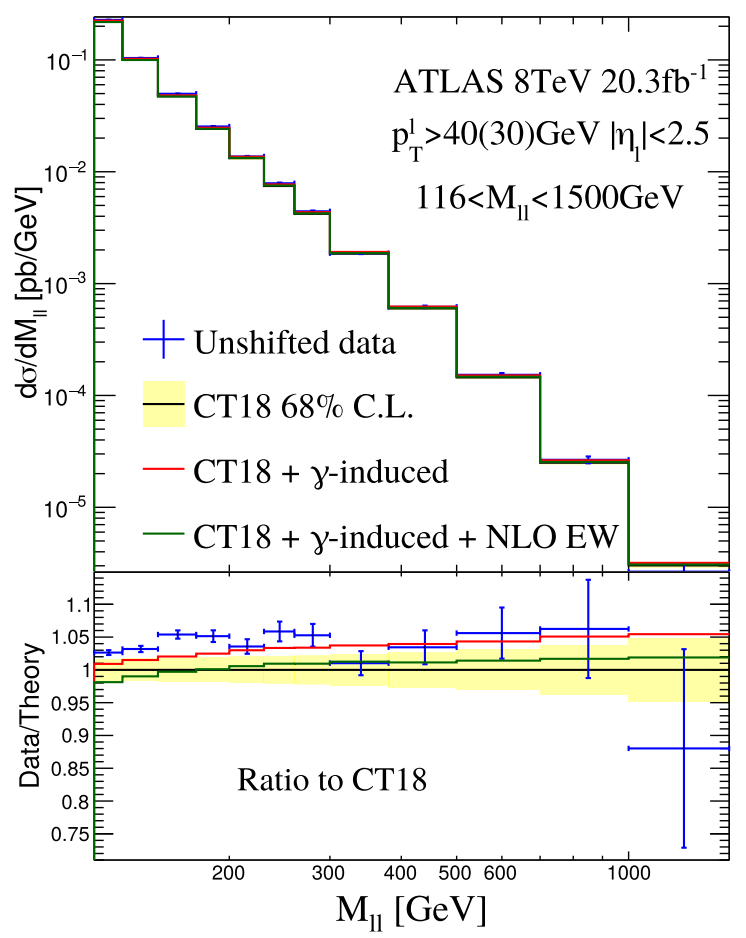

FIG. 46. The photon-induced contributions (photon PDF are taken from LUXqed17_plus_PDF4LHC15) and NLO EW corrections to ATLAS $8 \mathrm{TeV}$ high-mass Drell-Yan production.

CT18A(Z) fits. For the Z-peak and $W^{ \pm}$data, the EW corrections are included in the multiplicative $K$ factors, while the PI contribution is ignored. Finally, we note that, as discussed in Sec. 6.1.2 of Ref. [39], the background from the PI dilepton production has been subtracted from the ATLAS $7 \mathrm{TeV} W, Z$ data.

We also did not include the ATLAS $8 \mathrm{TeV}$ very highmass $\left(116<M_{\ell \bar{\ell}}<1500 \mathrm{GeV}\right)$ Drell-Yan data [181] in our CT18(Z) fitting, due to non-negligible EW corrections and PI contributions. We find that, for very high invariant masses $\left(M_{\ell \bar{e}} \sim 1 \mathrm{TeV}\right)$, the PI contribution can be as large as 5\% as computed with LUXqed17_plus_PDF4LHC15 [8]. In comparison, the EW corrections can be calculated using the FEWZ program as shown in Fig. 46, and are approximately $-3 \%$ in this case. The partial cancellation of the PI contribution and EW correction yields an increase in the cross section by less than $2 \%$. With the EPUMP program, we have also checked that the impact of these data on the CT18 fits is very small.

The only $Z p_{T}$ distribution included in the CT18(Z) fits comes from the ATLAS $8 \mathrm{TeV}$ measurements. We have dropped the high- $p_{T}$ data by imposing a kinematic cut of $p_{T}^{Z}<150 \mathrm{GeV}$ because the missing EW corrections to the high- $p_{T}$ data are significant. In general, we note that these corrections are negative. In terms of Refs. [134,135], the NLO EW corrections can be as large as several tens of percent when $p_{T}^{Z} \gg M_{Z}$, due to electroweak Sudakov logarithms. In the fitted region of $p_{T}^{Z}$, between 45 and
$150 \mathrm{GeV}$, the EW corrections are found to reduce the cross sections by several percent, thereby pulling the theory predictions further away from the ATLAS $8 \mathrm{TeV}$ data.

\section{STANDARD CANDLE CROSS SECTIONS}

Measurements of total cross sections for inclusive hadroproduction at colliders provide cornerstone tests of the Standard Model. These relatively simple observables can both be measured with high precision and predicted in NNLO QCD theory with small uncertainties. In Sec. VI A, we collect NNLO theory predictions, based upon the CT14, CT14 $1_{\text {HERAII }}$, and CT18(A/X/Z) NNLO PDFs, for the inclusive production cross sections of $W$ and $Z$ bosons, top-quark pairs, and Higgs bosons (through gluon-gluon fusion), at the LHC with center-of-mass energies of $\sqrt{s}=7,8,13$, and $14 \mathrm{TeV}$. These theoretical predictions supersede similar comparisons made with the previous generations of CT10/CT14 PDFs [1,42] and can be compared to the corresponding experimental measurements. In addition, we also present theoretical predictions for vector boson production at $\mathrm{LHCb}$ based on fixed-order and resummed calculations in Sec. VIB, explore predictions for $W+c$ production at ATLAS in Sec. VIC and $13 \mathrm{TeV} t \bar{t}$ production at CMS in Sec. VID, and show predictions for high- $x$ fixed-target Drell-Yan cross sections in Sec. VIE, in anticipation of the forthcoming results of the SeaQuest experiment [182] at Fermilab.

\section{A. Inclusive total cross sections at the LHC}

In this work, the masses of the top quark and Higgs boson are set to $m_{t}^{\text {pole }}=173.3 \mathrm{GeV}$ and $m_{H}=125 \mathrm{GeV}$, respectively. The $W$ and $Z$ inclusive cross sections (multiplied by branching ratios for the decay into one charged lepton flavor), are calculated by using the VRAP v0.9 program $[128,129]$ at NNLO in QCD, with the renormalization and factorization scales $\left(\mu_{R}\right.$ and $\left.\mu_{F}\right)$ set equal to the invariant mass of the vector boson. The total inclusive topquark pair cross sections are calculated with the help of the program TOP++ v2.0 $[183,184]$ at NNLO + NNLL accuracy, with QCD scales set to the mass of the top quark [136] as is the default in the TOP++ framework. The Higgs boson cross sections via gluon-gluon fusion are calculated at NNLO in QCD by using the IHIXS v1.3 program [185], in the heavyquark effective theory with finite top quark mass correction, and with the QCD scales set equal to the Higgs boson mass.

Figure 47 shows that the Higgs boson cross section through gluon-gluon fusion $(g g H)$ at the LHC does not have a pronounced correlation with the top-quark pair $(t \bar{t})$ cross section, because the two processes are dominated by the gluon PDF in somewhat different $x$ regions. The degree of anticorrelation found in the $g g H$ and $Z$ boson cross sections decreases as the LHC energy increases. On the other hand, Fig. 48 shows that the electroweak gauge boson cross sections are highly correlated with each other at the 

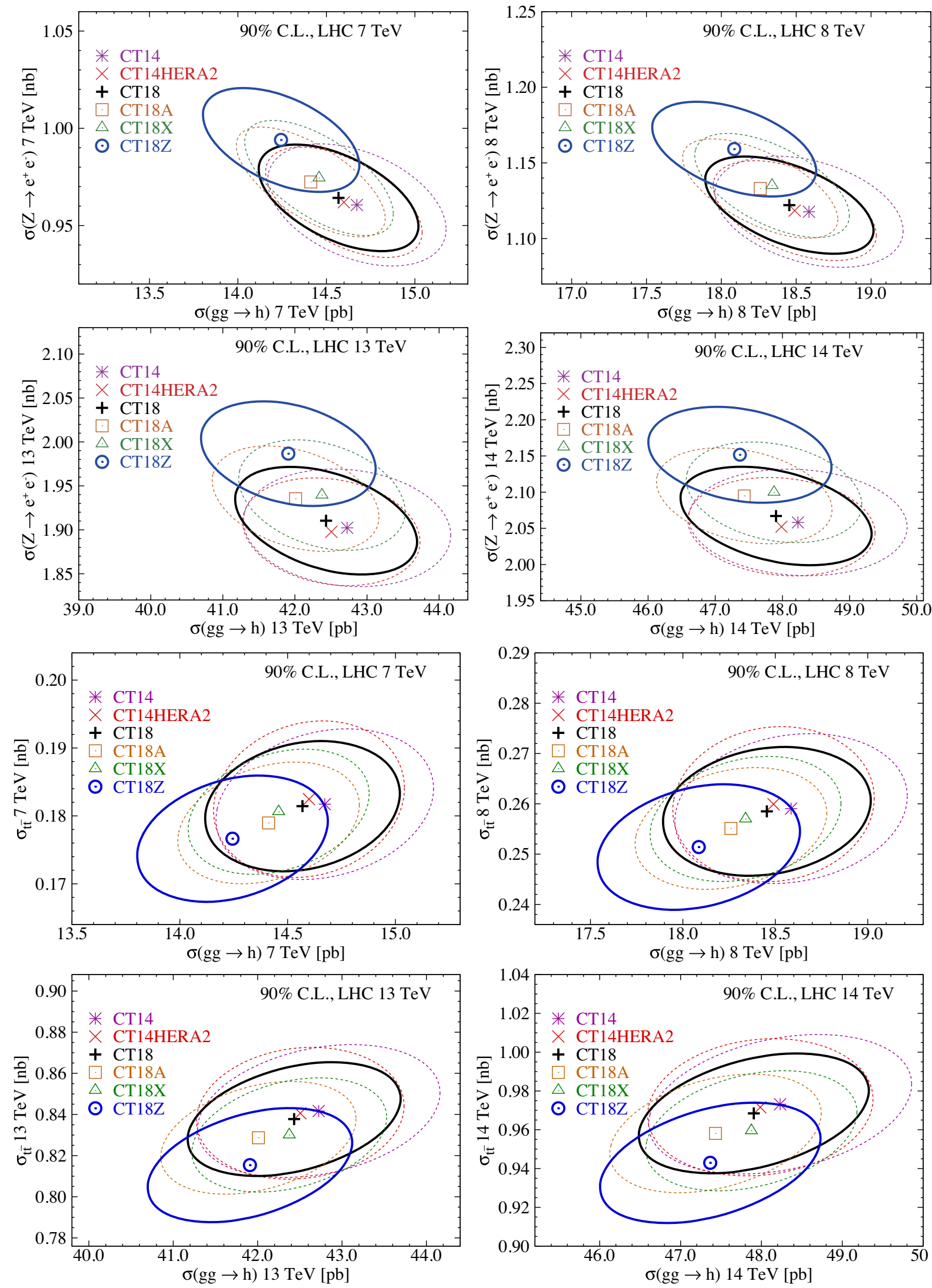

FIG. 47. The $90 \%$ C.L. error ellipses for the $g g H, t \bar{t}$, and $Z^{0}$ inclusive cross sections computed with the CT18 NNLO family of PDFs at the LHC 7, 8, 13, and $14 \mathrm{TeV}$. 

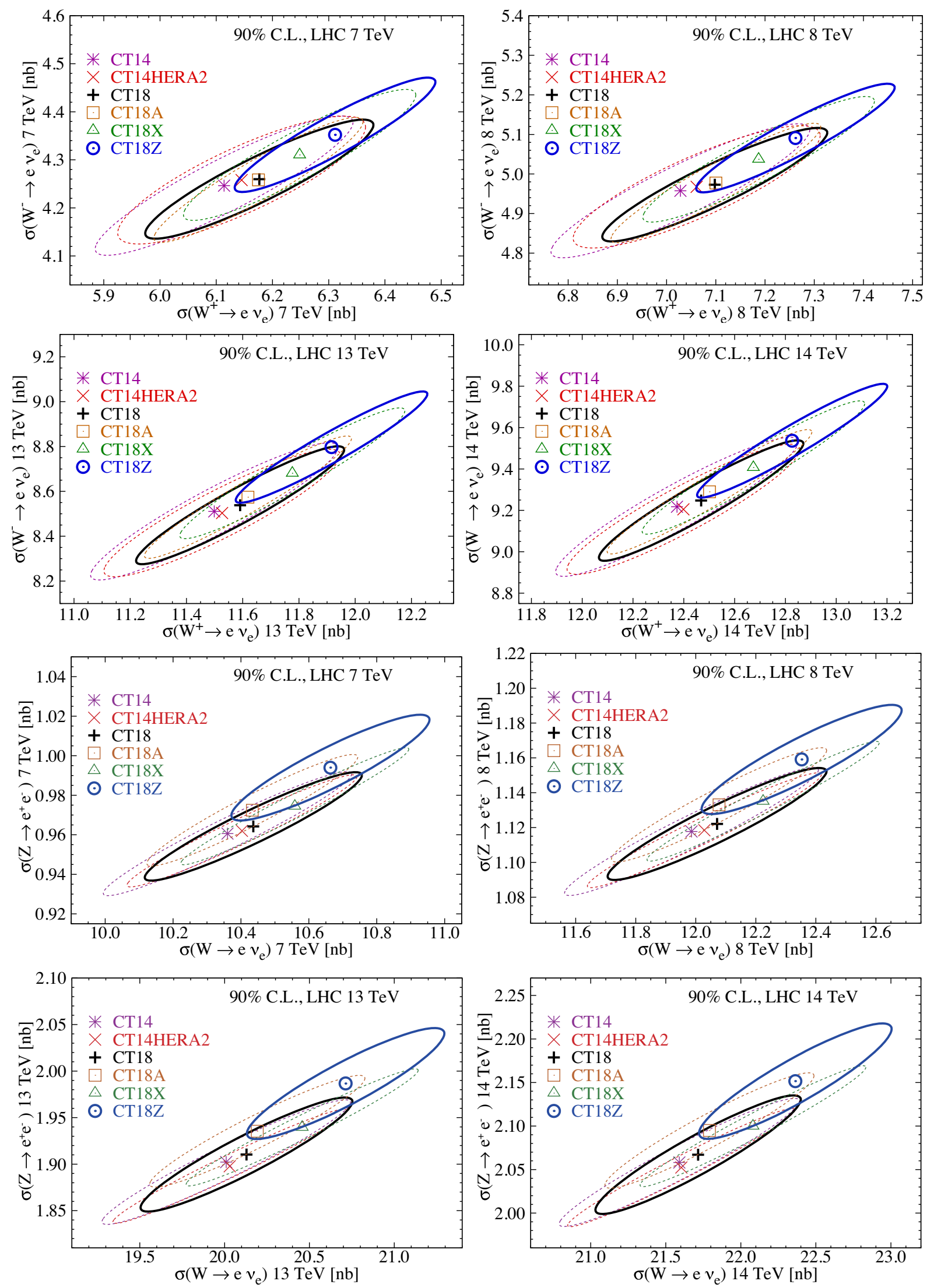

FIG. 48. Same as Fig. 47, but for the $W^{+}, W^{-}$, and $Z^{0}$ inclusive cross sections. 
LHC. Generally speaking, the prediction of CT18 is closer to $\mathrm{CT} 14_{\text {HERAII }}$, and the largest difference occurs between CT18Z and CT14. Furthermore, the CT18X prediction is closer to CT18Z for the electroweak gauge boson productions, cf. Fig. 48, but not for the $g g H$ or $t \bar{t}$ inclusive cross sections, cf. Fig. 47.

The mutual dispositions of the error ellipses for $W$ and $Z$ cross sections in the bottom half of Fig. 48 can be tied to the differences among the strangeness and other PDFs of the CT18, A, X, and Z ensembles discussed in Sec. A 2. In general, the orientations of all shown $W-Z$ ellipses are similar, with the direction parallel to the semiminor axesassociated with the relative difference between the $W$ and $Z$ production cross sections-most closely identified with the strange PDF. The correlation between the $s$ PDF and the ratio of $W^{ \pm}$to $Z$ cross sections was first pointed out in the CTEQ6.6 analysis [91]. The theory predictions based on CT18A and CT18Z are both equally shifted in this direction. Meanwhile, CT18X and, especially, CT18Z are significantly offset along the semimajor axis [the " $\sigma(Z)+\sigma(W)$ direction"], more related to the gluon at $x<10^{-2}$, as again was pointed out in [91]. The close alignment of CT18Z and A in the perpendicular direction relates closely to similarity in the fitted strangeness distributions obtained under these fits.

It is worthwhile to investigate whether the inclusion of nonperturbative charm may significantly alter these theoretical predictions, especially for electroweak boson production. Reference [3] suggested that tensions between the combined HERA data (Exp. ID = 160) and ATLAS $7 \mathrm{TeV}$ $W / Z$ data require that the charm PDF at the initial scale $Q_{0}$ be independently parameterized. Such nonperturbative charm, of indefinite sign and shape, was thus implemented using the unique neural network approach of the NNPDF collaboration as a "fitted charm" contribution to the proton's structure, distinct from perturbative charm. The question of intrinsic charm, including its dynamic origin in perturbative QCD, has also been studied by CT, most recently, in Ref. [150], which implemented positive nonperturbative charm as an explicitly twist-2 intrinsic PDF, informed by various models, at the scale $Q_{0}=m_{c}$ as a boundary condition for the perturbative evolution of charm.

Following this work, we show in Fig. 49 theoretical predictions for the total $W$ and $Z$ production cross sections at $7 \mathrm{TeV}$, analogous to the third left-hand panel of Fig. 48, but including several scenarios for IC. Correlation studies in the CTEQ6.6 analysis [91] have shown that the central point $\left\{\sigma_{W}, \sigma_{Z}\right\}$ is shifted in the direction $G$ in Fig. 48 primarily by increasing the gluon PDF in the relevant region $x \approx 0.01$. It is shifted in the direction $S$ primarily by increasing the $s$ PDF in a similar $x$ region.

By these rules of thumb, the upward shift of the ellipse for CT18A above CT18 is consistent with the increase of strangeness in CT18A upon the inclusion of the ATLAS $7 \mathrm{TeV} W / Z$ data. Inclusion of IC in CT14 shifts the

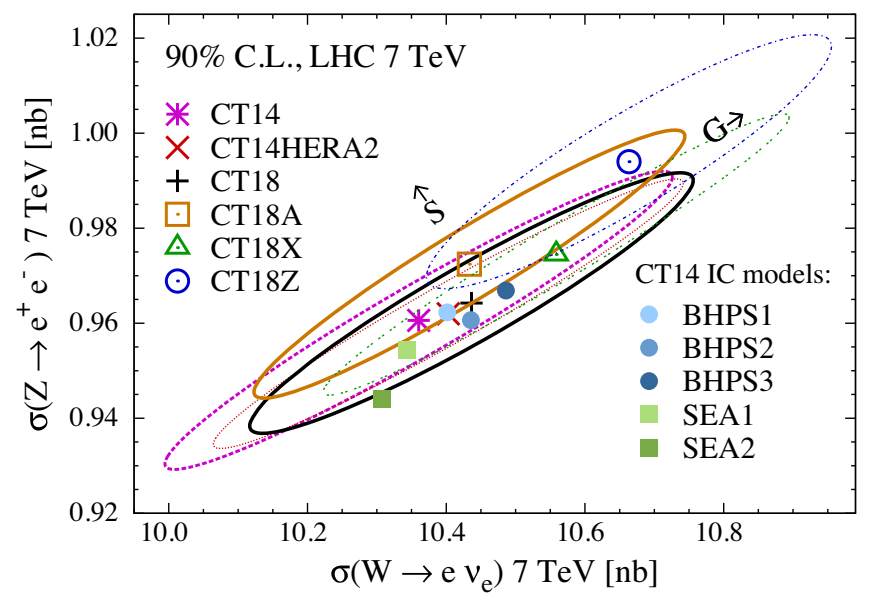

FIG. 49. Theoretical predictions of the total production cross sections of $W$ and $Z$ bosons at $\sqrt{s}=7 \mathrm{TeV}$ as relevant for the ATLAS $7 \mathrm{TeV} W / Z$ data (Exp. ID = 248). Here, we also include several calculations which include an intrinsic charm (IC) PDF based upon either the BHPS valencelike model (with three different normalizations) or a sealike model (with two different normalizations) in addition to CT14, as described in Ref. [150].

theoretical prediction for the central CT14 against the direction $S$, which is consistent with some suppression of strangeness preferred by DIS experiments in CT14 after a positive IC PDF is included. The CT14 IC predictions are also shifted along direction $G$ in reflection of the different magnitude of the gluon PDF in CT14 IC models, as compared to the nominal CT14.

The downward shift of the IC predictions in the figure, with respect to the purely extrinsic charm predictions of CT14, etc., thus appears to be a generic outcome of assuming a non-negative charm at the initial-scale $Q_{0}$, which would naturally arise from twist- 4 contributions as discussed in [150]. The reason is, again, some suppression of the strangeness PDF in such IC models, which only exacerbates the tension with the ATLAS $7 \mathrm{TeV} W / Z$ data. We conclude that the standard approach of including a nonnegative intrinsic $c_{\mathrm{IC}}\left(x, Q=m_{c}\right) \mathrm{PDF}$ is unlikely to resolve the tensions between ATLAS $W / Z 7 \mathrm{TeV}$ data and HERA. The subtleties involved in the definition and dynamical origin of intrinsic/fitted charm are sufficiently complex that more forthcoming analyses will be required to disentangle them and understand their phenomenological implications.

\section{B. Vector boson differential cross sections at the LHC}

As described previously, NNLO calculations have been used formerly to predict vector boson production data at both the Tevatron and LHC. In the past, we have also compared this type of precision data to ResBos predictions, which include effects from multi-gluon emission [55], to produce the CTEQ6.6, CT10, and CT14 PDFs. For this reason, it is important to compare vector boson differential 

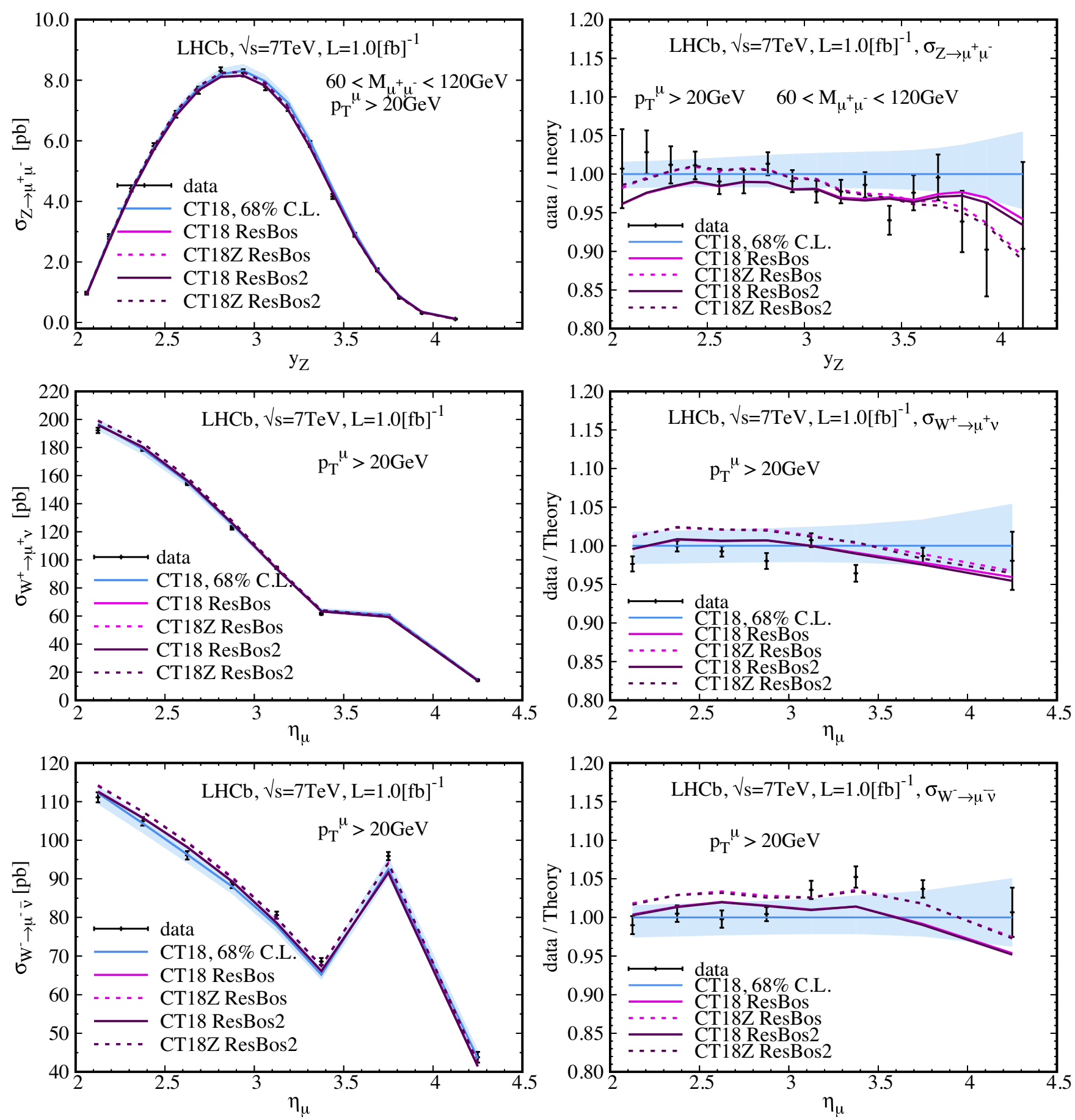

FIG. 50. Comparison of the LHCb $7 \mathrm{TeV} W$ and $Z$ data to CT18 predictions, with either NNLO (labeled as CT18), RESBOS (labeled by CT18 RESBOS) or RESBOS2 (labeled by CT18 RESBOS2) calculations. The prediction of CT18Z NNLO is also shown.

cross section measurements to predictions based on the CT18 (and CT18Z) PDFs with RESBOS and NNLO calculations. As an example, we compare the RESBOS predictions to the $\mathrm{LHCb} 7 \mathrm{TeV} W$ and $Z$ boson differential distributions [87] in Fig. 50.

For completeness, we have also included in the same figure the predictions from RESBOS2, which is an updated version of the RESBOS project to include full NNLO corrections, i.e., the complete $\alpha_{s}^{2}$ contribution for DrellYan production of the dilepton pair has been included in this calculation [186]. In contrast, the RESBOS prediction only contains parts of the NNLO contribution. More specifically, it includes only the Wilson coefficient $C^{(1)}$, but not $C^{(2)}$, in the resummation calculation, cf. Ref. [55]. As shown in Fig. 50, the predictions from RESBOS and RESBOS2 agree well for the LHCb kinematics, except in the 

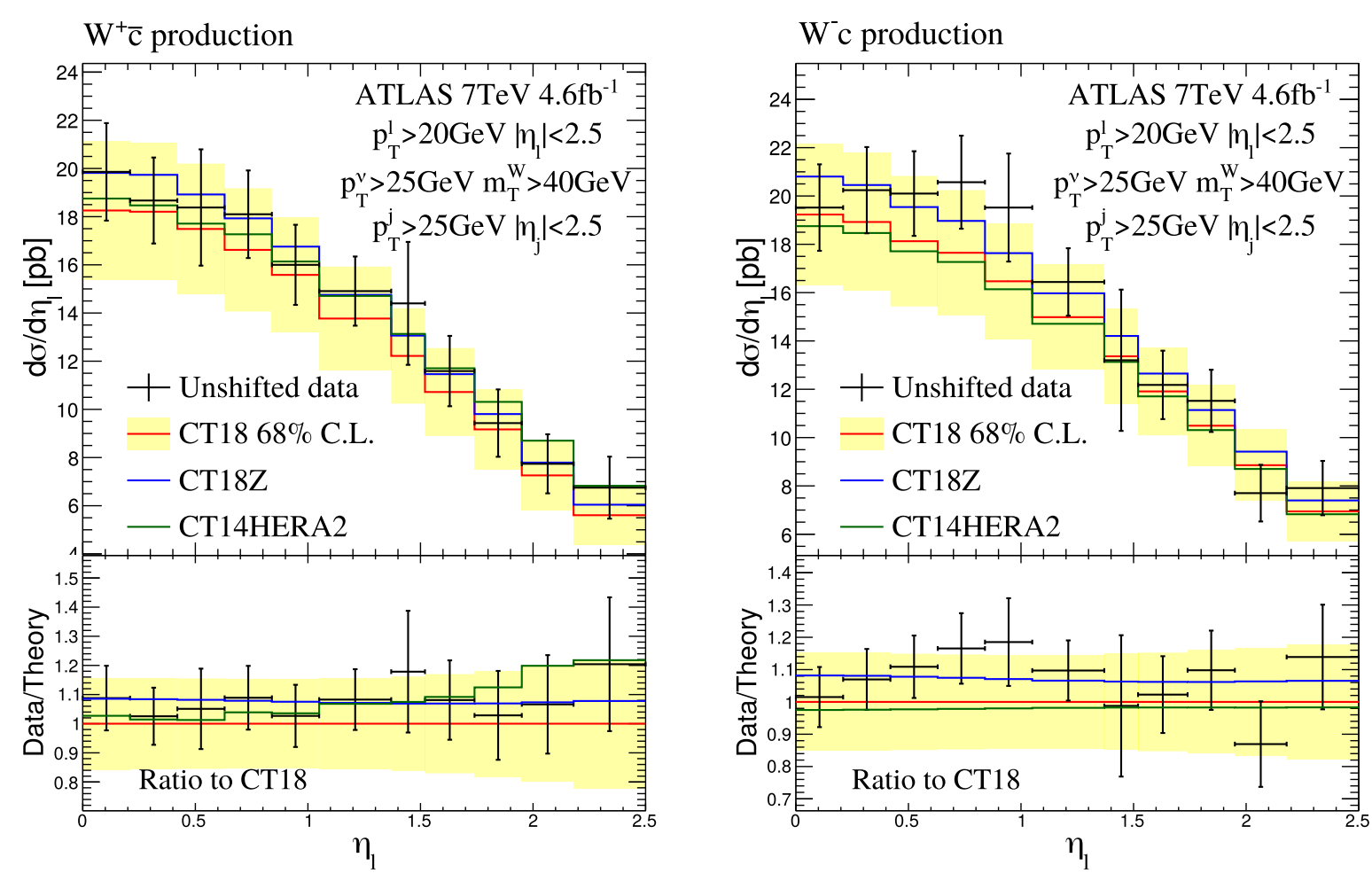

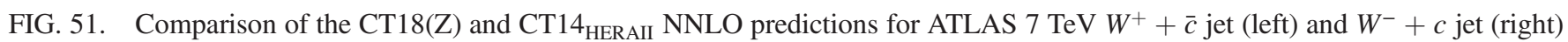
production, respectively, for the combined electron and muon decay channels. The CT18 PDF uncertainty is evaluated at $68 \%$ C.L. The scale choice is $\mu_{R}=\mu_{F}=M_{W}$.

very large rapidity region. The difference between the resummed and (NNLO) fixed-order predictions arises from multiple soft-gluon radiation, the effect of which tends to grow in the large-rapidity region, where it becomes comparable in size to the $\mathrm{LHCb} 7 \mathrm{TeV}$ experimental errors. Further detailed discussion about the difference between the resummation and fixed-order calculations will be presented elsewhere. In order to see how different PDFs might modify these comparisons between theory predictions and the LHCb $7 \mathrm{TeV}$ data, we also present in the same plot predictions based upon the CT18Z PDFs, in which the gluon and sea-quark distributions differ from those of CT18.

\section{C. $W$ plus charm-jet production at the LHC}

The $s$-quark PDF of CT18 differs from CT14 at $x<10^{-1}$ predominantly as a result of the inclusion of the new LHC vector boson production data from LHCb and ATLAS $7 \mathrm{TeV}$. Independent constraints on the strange quark are provided by the cross sections with significant contributions of processes initiated by $s$ quarks, such as $W$ plus charm-jet production [187]. As this process has not yet been calculated to NNLO, the relevant data samples have not been included in the CT18(Z) NNLO PDF fit, but it is still instructive to compare the NLO predictions (but with the NNLO PDFs) with the data.
Figure 51 compares the CT14 $4_{\text {HERAII }}$, CT18, and CT18Z predictions with ATLAS $7 \mathrm{TeV} W^{+}+\bar{c}$ jet and $W^{-}+c$ jet data, respectively. The PDF uncertainty is evaluated at 68\% C.L. and represented by the yellow bands. The theoretical calculations are performed by using APPLGRID tables generated with MCFM, cross checked against MadGraph_aMC@NLO + AMCFAST. The scale choice for this calculation is $\mu_{R}=\mu_{F}=M_{W}$ and the running of $\alpha_{s}$ is at NNLO as provided by the LHAPDF tables used together with APPLGRID. The $\chi^{2} / N_{p t}$ values are $0.59,0.52$, and 0.41 , respectively, with the CT14 HERAII, CT18, and CT18Z PDFs, for the total of $N_{p t}=22$ data points. We observe an upward shift in the predictions based upon CT18Z compared with CT18 in both panels of Fig. 51 for $W^{+}$ and $W^{-}$.

Interestingly, for $W^{+} \bar{c}$ production, the CT14 HERAII predictions tend to be even larger than those of CT18(Z) in the large-rapidity region, while for $W^{-} c$ production in the right panel of Fig. 51, the CT14 $4_{\text {HERAII }}$ predictions lie well below the CT18(Z) predictions over the full plotted range. This nuanced behavior of the large-rapidity $W^{+} c$ cross section reflects not only the increase of $s$-quark PDFs in CT18Z, but also some compensating changes in $g, d$, and other PDFs that occur at large $x$ and have been independently verified by updating the CT14 $4_{\text {HERAII }}$ PDFs using $W+c$ cross sections with the EPUMP program. For completeness, 

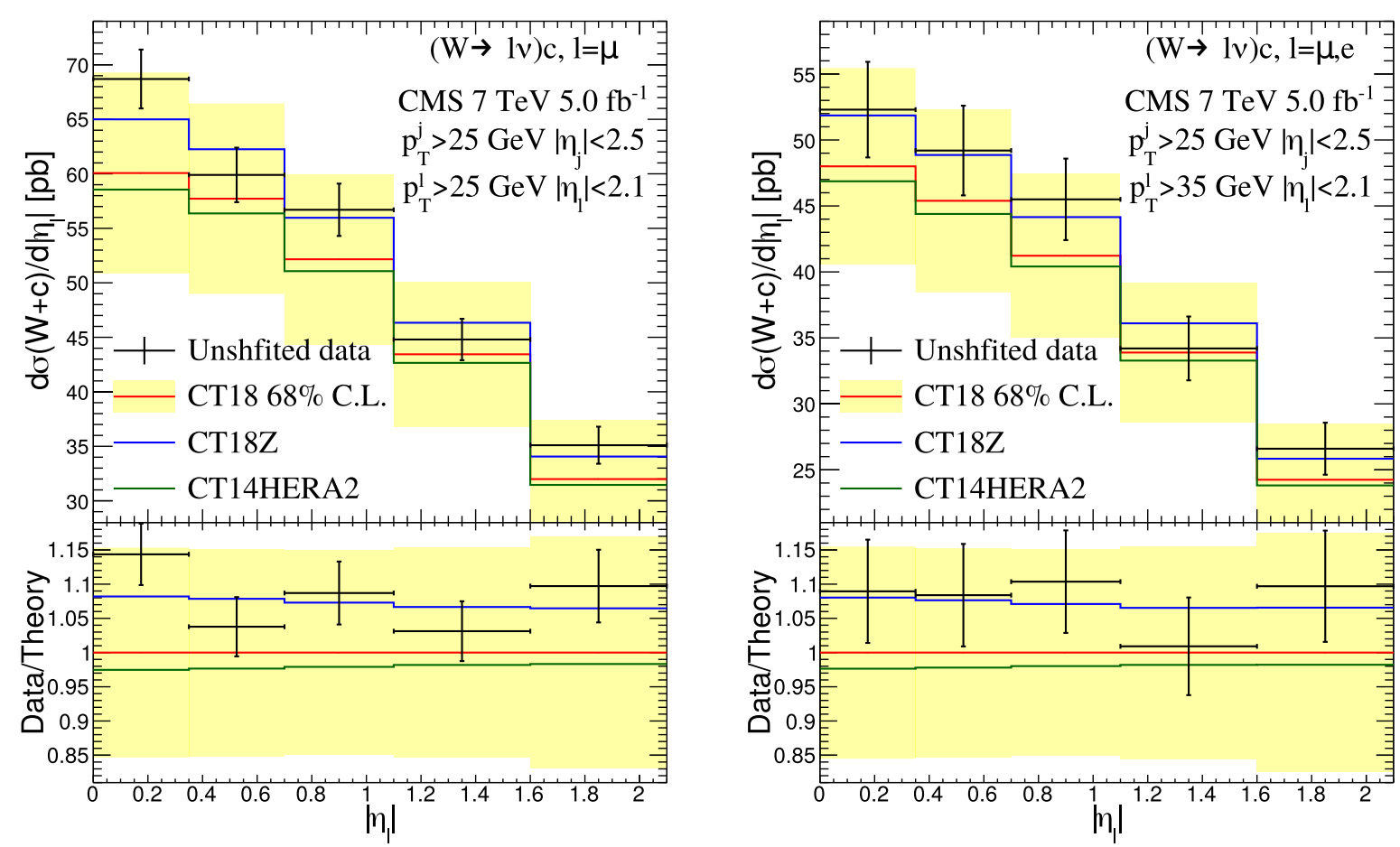

FIG. 52. Comparison of CT18(Z) and CT14 $14_{\text {HERAII }}$ predictions with CMS $7 \mathrm{TeV} W+c$ data, with lepton transverse momentum cut $p_{T}^{l}>25 \mathrm{GeV}$ (left), for the muon channel, and $p_{T}^{l}>35 \mathrm{GeV}$ (right), for the combined electron and muon channels, respectively. The scale choice is $\mu_{R}=\mu_{F}=M_{W}$.

we also show the similar comparison to CMS $7 \mathrm{TeV} W+c$ data in Fig. 52.

\section{Top quark pair differential distributions at the LHC $13 \mathrm{TeV}$}

In Sec. VB 3, we have shown data-to-theory comparisons to the ATLAS and CMS differential topproduction data at $8 \mathrm{TeV}$, i.e., data which were included in the $\mathrm{CT} 18(\mathrm{Z})$ fits. In this section we present analogous comparisons for the CMS $13 \mathrm{TeV}$ measurement of $t \bar{t}$ differential cross sections in the dilepton channel [103]. These data have been released after the CT18(Z) datasets were frozen in the final form. The QCD theoretical predictions at NNLO in QCD are obtained by using fastNNLO tables [18] with CT18 NNLO PDFs. The value of the top-quark mass used to obtain the theory predictions in this case is $m_{t}^{\text {pole }}=172.5 \mathrm{GeV}$. We also show the resultant theory predictions using CT18Z, CT18X, and CT18A NNLO PDFs, with PDF uncertainties for the cross sections shown at the $68 \%$ C.L. Plots of the distributions and data-vs-theory comparisons are shown in Figs. 53-55. In the data-vs-theory plots, all theory predictions are normalized to CT18NNLO theory. The error bars represent the quadrature sums of the statistical and total systematic
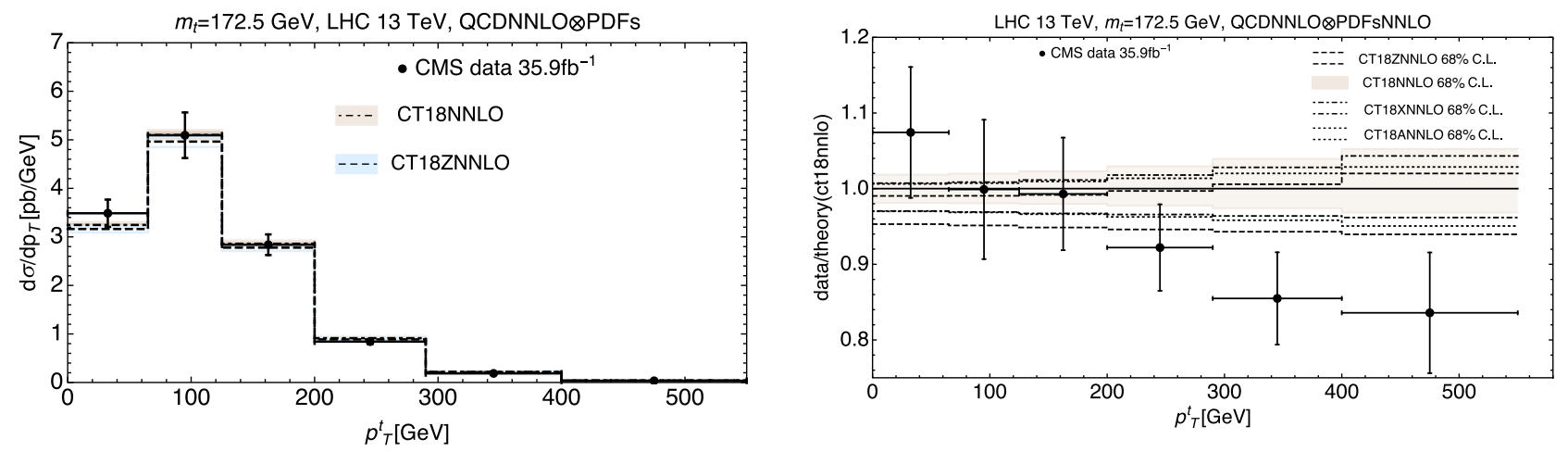

FIG. 53. Left: top-quark transverse momentum $p_{T, t}$ distribution. Right: unshifted data vs theory plot including CT18Z, CT18X, CT18A NNLO. In the right figure, data and theory predictions are normalized to CT18NNLO theory. The error bars indicate statistical and total systematic errors summed in quadrature. 

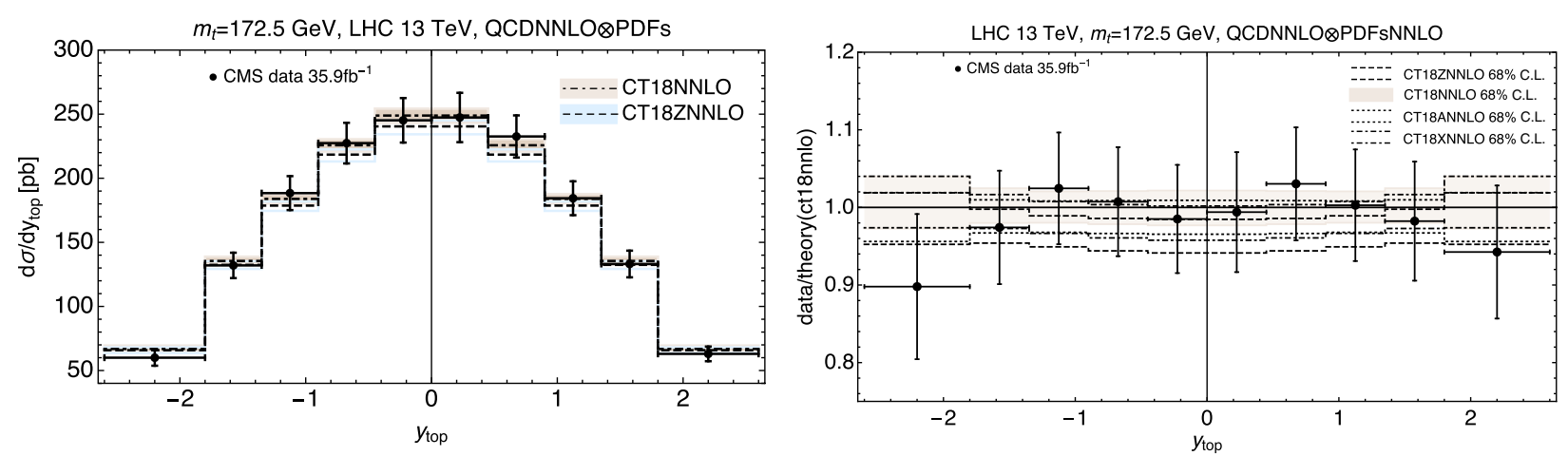

FIG. 54. Left: Top-quark rapidity $y_{t}$ distribution. Right: Unshifted data vs theory plot including CT18, CT18Z, CT18X, CT18A NNLO. Data and theory predictions are normalized to CT18NNLO theory. The error bars indicate statistical and total systematic errors summed in quadrature.
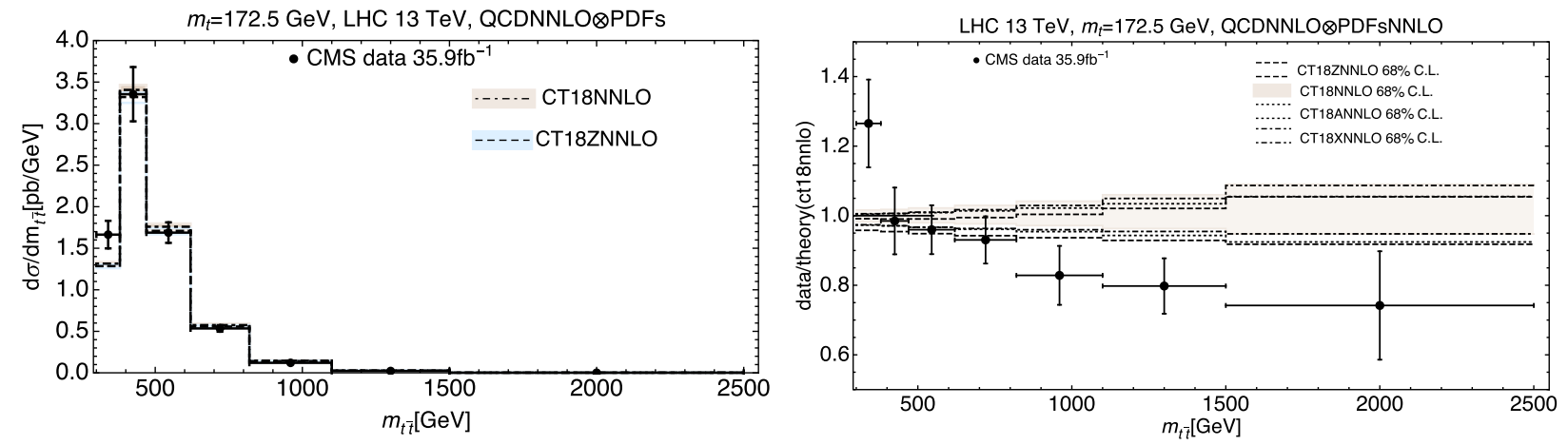

FIG. 55. Left: invariant mass distribution of the top-quark pair. Right: unshifted data vs theory plot including CT18, CT18Z, CT18X, CT18A NNLO. Data and theory predictions are normalized to CT18NNLO theory. The error bars are statistical and total systematic errors summed in quadrature.

errors. We observe a clear difference in the slope between the theory and unshifted experimental data for both $d \sigma / d p_{T}^{t}$ and $d \sigma / d m_{t \bar{t}}$. Those differences can be accommodated by systematic error shifts of the data, resulting in a good $\chi^{2}$ after all uncertainties are taken into account. We notice that, in the case of the $p_{T}$ spectrum, the theory prediction obtained with CT18Z NNLO gives a slightly better description of the data at large $p_{T}$.

The impact of the electroweak corrections on the CT18 theory is illustrated in Fig. 56. These corrections are included as $K$ factors using the multiplicative scheme according to Ref. [136]. They are available at [137].
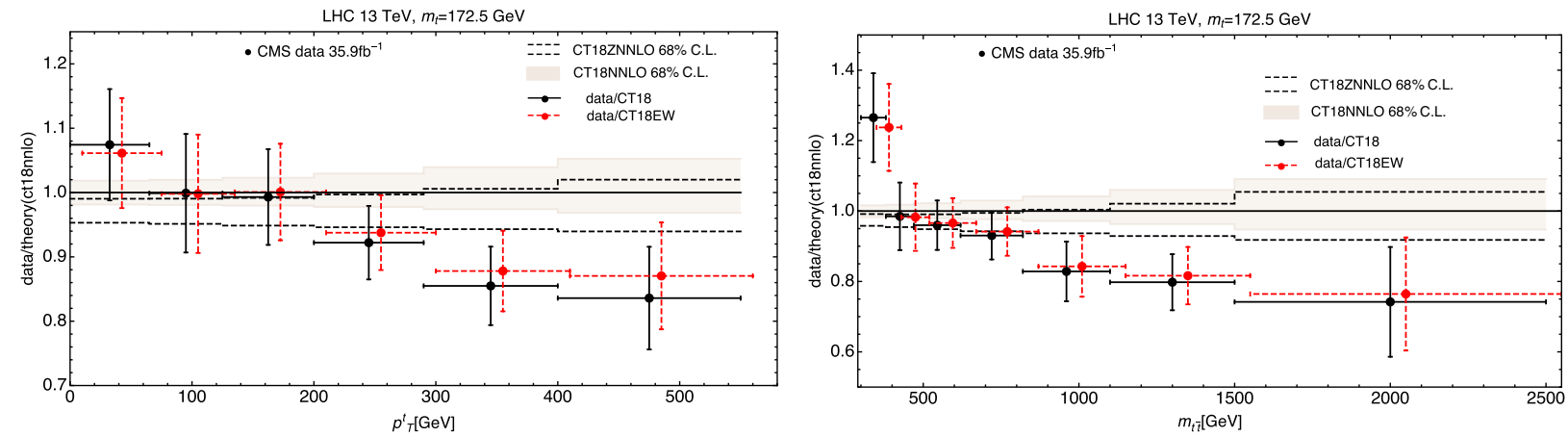

FIG. 56. Impact of NLO EW corrections. Unshifted data vs theory plot for top-quark $p_{T}$ distribution and the invariant mass distribution of the $t \bar{t}$ pair. The error bars are statistical and total systematic errors summed in quadrature. The red data points with dashed error bars represent data divided by the CT18NNLO theory with NLO EW corrections. The black data points with solid error bars represent data normalized to the CT18NNLO theory. The CT18ZNNLO theory prediction (black dashed error band) is also normalized to CT18NNLO. The data vs (theory + EW) points are slightly shifted to the right in the same bin to improve visualization. 

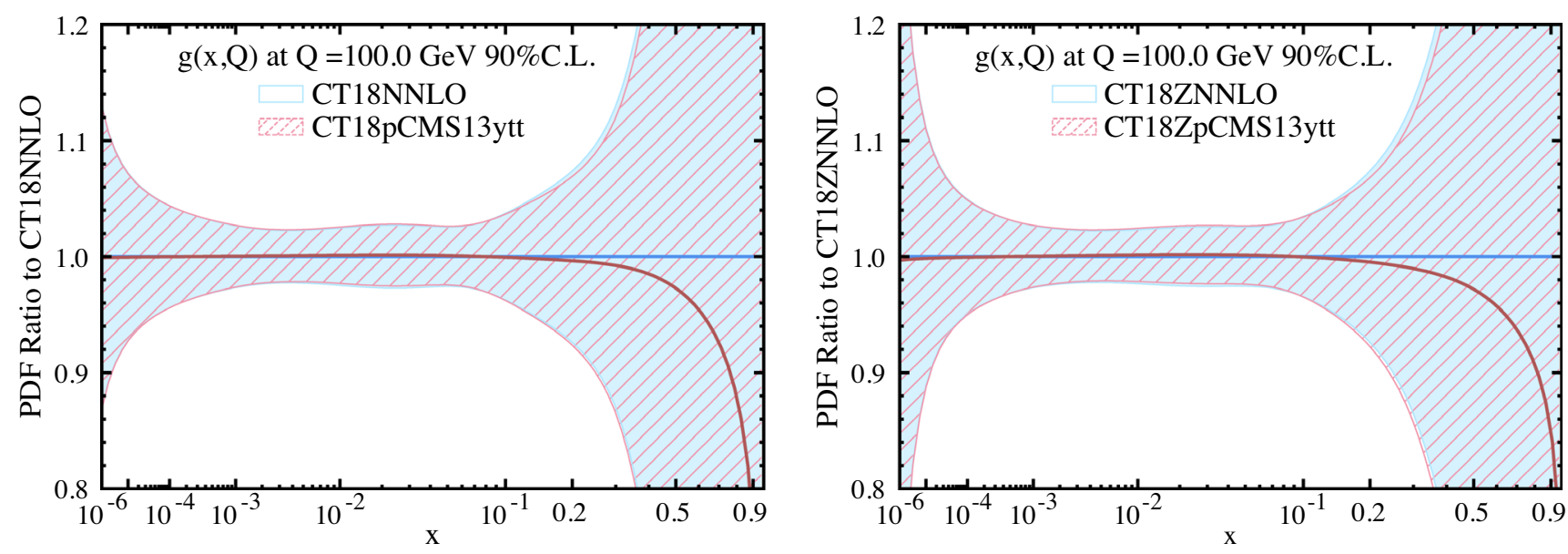

FIG. 57. Impact of the $y_{t \bar{t}}$ differential cross section measurements of the CMS $13 \mathrm{TeV} t \bar{t}$ data on the CT18 (left) and CT18Z (right) gluon PDFs.

Large EW effects show up in the high $p_{T}^{t}$ tails. However, in the $p_{T}$ range $1-500 \mathrm{GeV}$ shown in the figures, the EW corrections are not larger than $3-4 \%$ in most cases. If one considers higher- $p_{T}$ regions, $K$ factors would be much larger there. The EW corrections minimally improve the agreement of theory and data for the top-quark $p_{T}$ and $m_{t \bar{t}}$ distributions. The $\chi^{2} / N_{p t}$ of the NNLO QCD + NLO EW prediction using CT18 PDFs agrees well with the values presented in Table 49 of Ref. [103]. For all other distributions, the EW corrections are negligible for the kinematic ranges studied. The CT18 global analysis currently includes $\bar{t} t$ differential cross section measurements from ATLAS and CMS at $8 \mathrm{TeV}$ only. The CT18 theory prediction for these distributions in the fit does not include EW corrections. If EW corrections were included in the fit their impact on the fitted PDFs would be negligible due to the size of the EW corrections in the kinematic range of the distributions currently considered.

Among various one-dimensional $t \bar{t}$ differential distributions, the distribution of the top-quark pair rapidity, $y_{t \bar{t}}$, shows a good agreement between the CMS data and CT18 predictions. To examine how this data could modify the CT18(Z) gluon PDFs, we use the EPUMP program [25] to update the CT18(Z) PDFs, after including the CMS $13 \mathrm{TeV}$ $y_{t \bar{t}}$ data in the fit. As shown in Fig. 57, the updated gluonPDF error band (labeled as CT18 pCMS13ytt) is very slightly reduced for $x$ from 0.1 to 0.4 in both cases. Further discussion about these datasets will be presented elsewhere.

\section{E. High- $x$ Drell-Yan predictions}

Fixed-target Drell-Yan measurements provide an important probe of the $x$ dependence of the nucleon (and nuclear) PDFs. This fact has motivated a number of experiments, including the Fermilab E866/NuSea experiment [68], which determined the normalized deuteron-to-proton cross section ratio $\sigma_{p d} / 2 \sigma_{p p}$ out to relatively large $x_{2}$, the momentum fraction of the target. As can be seen based upon a leading-order quark-parton model analysis, this ratio is expected to have especially pronounced sensitivity to the $x$ dependence of the PDF ratio, $\bar{d} / \bar{u}$, making it a favorable observable for investigations of flavor-symmetry breaking in the light-quark sea. Breaking of SU(2) symmetry is understood to have a nonperturbative origin, as noted in the discussion of the Gottfried sum rule in Sec. IV D.

Intriguingly, E866 [68] found evidence that the cross section ratio dropped below unity, $\sigma_{p d} / 2 \sigma_{p p}<1$, as $x_{2}$ approached and exceeded $x \gtrsim 0.25$, as seen by the higher $x_{2}$ portion of the E866 ratio points shown in Fig. 58. This fact was surprising on the grounds of a number of theoretical models. The E866 results therefore stimulated

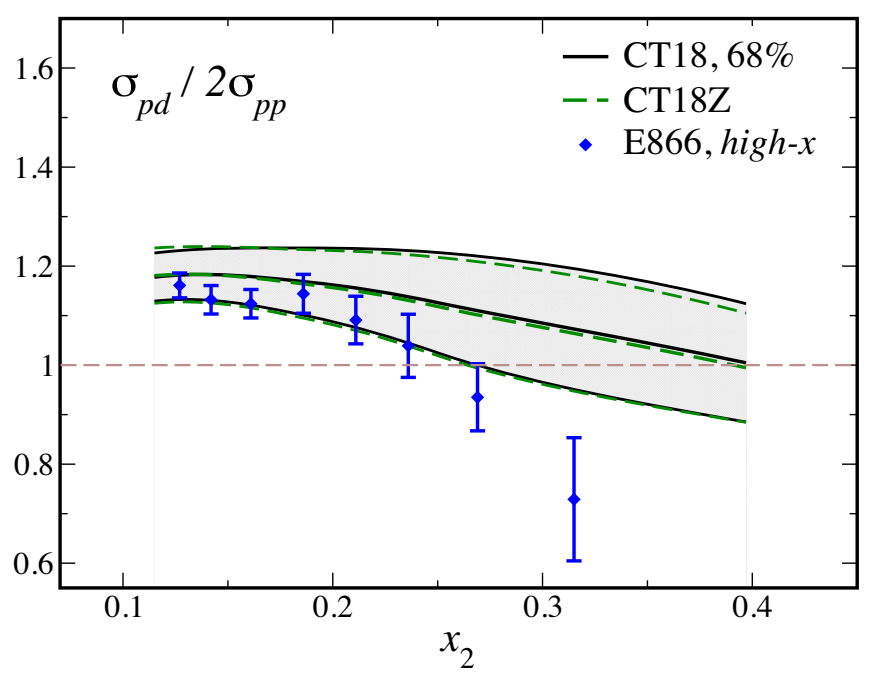

FIG. 58. Theoretical predictions based on CT18 (black outer band) and CT18Z (green inner band) for the fixed-target DrellYan cross section, $\sigma_{p d} / 2 \sigma_{p p}$, in the region of larger $x_{2} \gtrsim 0.1$ to be probed by the SeaQuest experiment [182] at Fermilab. For comparison, we also plot the higher- $x_{2}$ portion of the older E866 data [68] (blue diamonds). 
an interest in performing a similar measurement out to larger $x_{2}$ with higher precision - the main objective of the subsequent SeaQuest/E906 experiment at Fermilab [182], from which results are expected soon. For this reason, we illustrate in Fig. 58 theoretical predictions based upon our updated CT18 (black band) and CT18Z (green band) global analyses at the $68 \%$ C.L. to higher $x_{2}$ beyond that probed by E866. While CT18 and CT18Z are constrained to the E866 ratio data, the theoretical prediction for the deuteronto-proton ratio remains above or consistent with unity out to $x<0.4$. More precision data in the high- $x$ region will be instrumental in resolving the behavior of the cross section ratio and its implications for the nucleon sea.

\section{DISCUSSION AND CONCLUSIONS}

In this paper, we have presented the CT18 family of parton distribution functions (PDFs), including the CT18Z, CT18A, and CT18X alternative fits. CT18 is the next generation of NNLO (as well as NLO) PDFs of the proton from a global analysis by the CTEQ-TEA group. It represents the next update following the release of the CT14 and CT14 $4_{\text {HERAII }}$ NNLO distributions, the latter of which was prompted by the release of precision HERA I and II combined data after the publication of CT14. CT18 is the nominal CTEQ-TEA PDF set, which we recommend for all general-use applications. CT18A is the product of adding the ATLAS $7 \mathrm{TeV} W / Z$ data [39] into the CT18 fit; CT18X is a variation of CT18 with an $x$-dependent QCD scale for the low- $x$ DIS data (along with a slightly larger charm quark mass value of $1.4 \mathrm{GeV}$ ); and CT18Z contains all the above variations and generally differs most significantly from CT18. The differences between the CT18 and CT18Z datasets will be non-negligible only for a small range of applications, in which these differences will need to be folded into the total PDF uncertainty, for example, by taking the envelope of the CT18 and CT18Z uncertainties. CT18A can be used for more complete examination of the range of uncertainty for the strange quark distribution. Similarly, the possible impact of low $x$ resummation can be explored using CT18X.

Although some of the early 7 and $8 \mathrm{TeV}$ LHC run- 1 data, including measurements of inclusive production of vector bosons [75-77,94] and jets [95,96], were included as input for the CT14 fits, CT18 represents the first CT analysis that substantially includes the most important experimental data from the full run-1 of the LHC, including measurements of inclusive production of vector bosons, jets, and top quark pairs at 7 and $8 \mathrm{TeV}$. Detailed information about the specific datasets included in the CT18 global analysis can be found in Tables I and II, with the newly included data in the latter table. With rapid improvements in the precision of LHC measurements, the focus of the global analysis has shifted toward providing accurate predictions in the wide range of $x$ and $Q$ covered by the LHC data, by making use of the state-of-the-art theory calculations. To achieve this goal requires a long-term multipronged effort in theoretical, experimental, and statistical areas.

The challenge from the side of the experimental information is to select and implement relevant and consistent datasets in the global analysis. Specifically, we have included processes that have a sensitivity for the PDFs of interest, and for which NNLO predictions are available. For example, we include as large a rapidity interval for the ATLAS jet data as we can, using the ATLAS decorrelation model, rather than using a single rapidity interval. We noted that using a single rapidity interval may result in selection bias. The result may be a larger value of $\chi^{2} / N_{p t}$, due to remaining tensions in the ATLAS jet data, as well as reduced PDF sensitivity compared to the CMS jet data, cf. Sec. II B 4. Similarly, to incorporate the $t \bar{t}$ differential cross section measurements into the CT18 global analysis, we use two $t \bar{t}$ single differential observables from ATLAS (using statistical correlations) and doubly differential measurements from CMS in order to include as much information as possible. Again, there is a risk of bias if we were to use only one differential distribution; however, some of the $t \bar{t}$ observables are in tension with each other, cf. Sec. II B 5. The CT18 global analysis shows that previous datasets, included in the CT14 global analyses, continue to have very strong pulls and tend to dilute the impact of new data. For example, low-energy DIS and Drell-Yan data, precision HERA data and precise measurements of the electron-charge asymmetry from $D \varnothing$ at $9.7 \mathrm{fb}^{-1}$ [78] remain important for probing combinations of quark flavors that cannot be resolved by the LHC run-1 data alone. Furthermore, most experimental measurements contain substantial correlated systematic uncertainties; we have taken account of these systematic errors in examining the PDF impacts of these measurements. In addition, we have examined the PDF errors for important LHC processes and have tested the consistency of the Hessian and LM approaches.

The challenges from the theoretical side are threefold: to examine the dependence of theoretical predictions upon QCD-scale choices in comparison with experimental precision, to explore the impact on the global analysis and uncertainty in the chosen parametrization forms for the nonperturbative PDFs, and to be able to do fast and accurate theory calculations. In the nominal CT18 fits, we have used the canonical choice of the QCD renormalization and factorization scales, which typically stabilize higher-order theoretical corrections. Fits with alternative scale choices were considered when studying the PDF uncertainty, cf. Sec. III C 3.

For the CT18 NNLO PDFs in particular, we have consistently applied NNLO calculations to precision DIS, Drell-Yan, jet and $t \bar{t}$ processes, cf. Sec. III. The specific QCD-scale choices we take for various processes are listed in Tables IV and V. For example, a non-negligible difference was found at low-jet transverse momentum between theory 
predictions at NNLO using as the momentum-scale choice either the inclusive jet or the leading-jet transverse momentum [13]. The nominal choice adopted by the CTEQ-TEA group is to use the inclusive-jet $p_{T}$. We have observed that the fitted gluon PDF is not very sensitive to this choice even in kinematic regions where the difference in predictions between these two scale choices is important. This resilience in the global fit is due to the presence of other data constraining the gluon PDF in the relevant kinematic region and possibly due to the compensating effects from sizable systematic uncertainties. To compare with the high precision data at the LHC, electroweak corrections must also be included in theory predictions. Details can be found in Sec. III B, cf. Table VIII.

To examine the dependence of the fits upon the nonperturbative functional forms chosen for the PDFs at the evolution starting scale $Q_{0}$ (around $1.3 \mathrm{GeV}$ ), we have sampled a large, $\mathcal{O}(250)$, collection of candidate fitting forms, all having a comparable number of fitting parameters. (More flexible parametrizations are used to better capture variations in the PDFs' $x$ dependence, $\mathrm{cf}$. Appendix C.) The result of this study can be seen in Fig. 6. As we increase the number of fitting parameters in the global analysis, we typically observe a steady improvement in $\chi^{2}$; this improvement generally increases so long as $\lesssim 30$ parameters are fitted, beyond which fits tend to destabilize as expanded parametrizations attempt to describe statistical noise.

In order to perform the CT18 global fits at NNLO for comparison with precision data whose per datum statistical error can be as small as $0.1 \%$, we require fast theory calculations with high numerical precision. Hence, the usage of various fast interfaces on the calculations of structure functions and cross sections becomes mandatory and conventional. For that, we have internally developed fast APPLGRID and fastNLO calculations at NNLO accuracy in the QCD interaction, cf. Sec. III. In addition, we have also parallelized our global-fitting algorithms to facilitate greatly accelerated convergence times, as discussed in Appendix D.

The experimental collaborations at the LHC have succeeded in taking copious high-precision data. To examine the agreement with these precision data calls for advances in statistical methodology. Which of the eligible LHC experiments provide promising constraints on the CTEQTEA PDFs? Do the LHC experiments agree among themselves and with other experiments? A consistent answer emerges from a powerful combination of four methods: (1) PDFSENSE and $L_{2}$ sensitivity, (2) the EPUMP program, (3) Effective Gaussian variables, and (4) LM scans. While the last two methods had been introduced in the previous CTEQ-TEA global analysis, such as CTEQ6, CT10, and CT14, the first two techniques were invented in the process of CT18 global analysis.

The PDFSENSE program [20] provides an easy way to visualize the potential impact of data on PDFs in the $x$ and
$Q$ plane. In addition, a simple $L_{2}$ sensitivity variable [21] is instructive for exploring agreement between different experiments similarly to the LM scans, but using a much faster Hessian formalism across the full range of $x$ or $Q$. See examples in Secs. VA 3 and A.

The complementary EPUMP program [24] contains a fast and efficient method to estimate the effect of new data on a set of best-fit and Hessian error PDFs. Extensive validations against the previous CT14 global fits have also been performed [25]. The application of the above four techniques in the CT18 analysis is illustrated throughout this paper.

In the four CT18 fits, important impacts are found on PDFs from ATLAS and CMS inclusive jet production measurements, LHCb $W$ and $Z$ vector boson productions and ATLAS $\sqrt{s}=8 \mathrm{TeV} \mathrm{Z}$ boson transverse momentum data. We find contradictory preferences for the strange quark PDF between semi-inclusive (SI) DIS (e.g., NuTeV and CCFR dimuon production) experiments, on one hand, and some LHC experiments, especially ATLAS $7 \mathrm{TeV}$ $W / Z$ production measurements and to some extent $\mathrm{LHCb}$ $W / Z$ measurements, on the other hand. Benchmarking of LHC measurements and theoretical predictions, as well as new (SI)DIS experiments can be highly effective for resolving these tensions. Going forward, to facilitate the discovery program of the high-luminosity run of the LHC, a sustained effort to navigate experimental tensions in collider data will be required to achieve the ultimate precision of these planned experiments. We envision an interplay among theoretical and data-analytical methods (including those used in this study to explore data compatibility), and additional high-precision experiments such as high-luminosity DIS colliders like the Electron-Ion Collider (EIC) [188], to be indispensable for making such progress.

The inclusion of new data and theoretical advances have resulted in the following changes in CT18, as compared to CT14: (1) a smaller $g(x, Q)$ for $x \sim 0.3$ (mainly due to ATLAS and CMS $7 \mathrm{TeV}$ jet data and ATLAS $8 \mathrm{TeV} Z p_{T}$ data, with some tension found between CMS 7 and $8 \mathrm{TeV}$ jet data), (2) some changes in $u, d, \bar{u}$, and $\bar{d}$ at small $x$, such as a larger $d$ and $d / u$ and a smaller $\bar{d} / \bar{u}$ for $x \sim 0.2$ (mainly due to LHCb $W$ and $Z$ rapidity data and CMS $8 \mathrm{TeV} W$ lepton charge asymmetry data), and (3) a larger $s$ and $(s+\bar{s}) /(\bar{u}+\bar{d})$ at small $x$ (mainly due to $\mathrm{LHCb}$ $W$ and $Z$ rapidity data, and further enhanced by the ATLAS $7 \mathrm{TeV} W / Z$ data in the CT18A and CT18Z fits). While the sensitivity of an individual $t \bar{t}$ data point can be similar to that of an individual jet data point at the LHC, the total sensitivity of the $t \bar{t}$ data is small due to the small number of $t \bar{t}$ data points. Hence, we did not find noticeable impact from the double differential distributions of the $t \bar{t}$ data included in the CT18 analysis. A similar finding was also reported in Ref. [99], in a CT14 analysis. 
Despite these changes in central predictions, the CT18 NNLO PDFs remain consistent with CT14 NNLO within the respective error bands. More details about the comparison of CT18 and CT14 PDFs, as well as the quality of the fits to data can be found in Secs. IV and V.

Some implications of CT18 predictions for phenomenological observables were reviewed in Sec. VI. Compared to calculations with CT14 NNLO, both the $g g \rightarrow H$ and $t \bar{t}$ total cross sections have decreased slightly in CT18. The $W$ and $Z$ cross sections, while still consistent with CT14, have slightly increased as a result of enhanced strangeness. Common ratios of strange and nonstrange PDFs for CT14 NNLO, shown in Sec. IVA 2, are consistent with the independent ATLAS and CMS determinations within the PDF uncertainties.

We have also presented the implications of the CT18 global fits for the value of $\alpha_{s}$, as seen in Sec. IV B. The full CT18 dataset prefers, at NNLO, a value of $\alpha_{s}\left(M_{Z}\right)=$ $0.1164 \pm 0.0026$, at $68 \%$ C.L. The corresponding value for CT18Z is basically the same, $0.1169 \pm 0.0027$. This is to be compared to the CT14 determination, which included very little LHC data, of $\alpha_{s}\left(M_{Z}\right)=0.115_{-0.004}^{+0.006}$ at $90 \%$ C.L.

The LM scans over the charm quark (pole) mass, $m_{c}$, as shown in Figs. 15 and 71, support the usage of $1.3 \mathrm{GeV}$ and $1.4 \mathrm{GeV}$ in the CT18 and CT18Z fits, respectively. Notably, the combined HERA charm data prefer a somewhat smaller $m_{c}$ value, while the ATLAS $7 \mathrm{TeV} W / Z$ data in the CT18Z fit prefer a larger $m_{c}$ value. Comments about the impact of fitted charm contributions on predictions for LHC $W / Z$ cross sections are made at the end of Sec. VIA. Comparisons to the parton luminosities and predictions based on the PDFs from other groups can be found in Secs. IV C, IV D, and A 3 a.

To allow direct comparison to results obtained by the lattice QCD community, we have also presented the CT18 predictions for various PDF moments and sum rules in Sec. IV D. In general, we find good agreement between CT18 and results from other phenomenological fitting efforts for most lattice observables. At present, systematic effects are such that many lattice calculations significantly overshoot the predictions of contemporary phenomenology, with the exception of the gluonic moment $\langle x\rangle_{g}$, which is underpredicted by the lattice relative to PDF fits. We expect complementary advances in lattice simulations and PDF phenomenology to improve this situation in coming years and pave the way for a synergistic PDF-lattice effort $[21,153]$ to determine the nucleon's longitudinal structure.

The final CT18 PDFs are presented in the form of 1 central and 58 Hessian eigenvector sets at NNLO and NLO. The $90 \%$ C.L. PDF uncertainties for physical observables can be estimated from these sets using the symmetric [23] or asymmetric $[38,90]$ master formulas by adding contributions from eigenvector pairs in quadrature. These PDFs are determined for the central QCD coupling of $\alpha_{s}\left(M_{Z}\right)=$ 0.118 , consistent with the world-average $\alpha_{s}$ value. For estimation of the combined $\mathrm{PDF}+\alpha_{s}$ uncertainty, we provide two additional best-fit sets for $\alpha_{s}\left(M_{Z}\right)=0.116$ and 0.120 . The $90 \%$ C.L. variation due to $\alpha_{s}\left(M_{Z}\right)$ can be estimated as a one-half of the difference in predictions from the two $\alpha_{s}$ sets. The PDF $+\alpha_{s}$ uncertainty, at 90\% C.L., and including correlations, can also be determined by adding the PDF uncertainty and $\alpha_{s}$ uncertainty in quadrature [29]. Aside from these general-purpose PDF sets, we provide a series of $(\mathrm{N}) \mathrm{NLO}$ sets for $\alpha_{s}\left(M_{Z}\right)=0.111-0.123$ and additional sets using heavy-quark schemes other than our standard five-flavor method, with up to three, four, and six active flavors.

Parametrizations for the CT18 PDF sets are distributed in a standalone form via the CTEQ-TEA website [189], or as a part of the LHAPDF6 library [190]. For backward compatibility with version 5.9.X of LHAPDF, our website also provides CT18 grids in the LHAPDF5 format, as well as an update for the CTEQ-TEA module of the LHAPDF5 library, which must be included during compilation to support calls of all eigenvector sets included with CT18 [191].

\section{ACKNOWLEDGMENTS}

We are indebted to our friend and colleague Jon Pumplin for decades of fruitful collaboration and for his last major contribution to the CTEQ-TEA global analysis made in this article. We thank Stefano Camarda, Amanda CooperSarkar, Alexander Glazov, Lucian Harland-Lang, Jan Kretzschmar, Bogdan Malescu, Wally Melnitchouk, Dave Soper, and CTEQ colleagues for insightful discussions. This work is partially supported by the U.S. Department of Energy under Grants No. DE-SC0010129 (at SMU) and No. DE-FG02-95ER40896 (at U. Pittsburgh); by the U.S. National Science Foundation under Grants No. PHY-1719914 and No. PHY-2013791 (at MSU) and No. PHY-1820760 (at U. Pittsburgh), and in part by the PITT PACC. T. J. Hobbs acknowledges support from a JLab EIC Center Fellowship. The work of M. Guzzi is supported by the National Science Foundation under Grant No. PHY-1820818. The work of J. G. was supported by the National Natural Science Foundation (NNSF) of China under Grants No. 11875189 and No. 11835005, and the work of S. D. and I. S. under NNSF Grants No. 11965020 and No. 11847160. C.-P. Yuan is also grateful for the support from the Wu-Ki Tung endowed chair in particle physics.

\section{APPENDIX A: THE ALTERNATIVE CT18Z GLOBAL FIT}

In this Appendix, we describe a series of fits leading to the CT18Z PDFs that provide a distinct alternative to the primary result of this analysis, CT18 NNLO. While CT18Z NNLO achieves a comparable level of success in describing the CTEQ-TEA data, producing $\chi^{2} / N_{p t}=1.19$ as opposed to $\chi^{2} / N_{p t}=1.17$ for the CT18 NNLO fit, the quality of the 


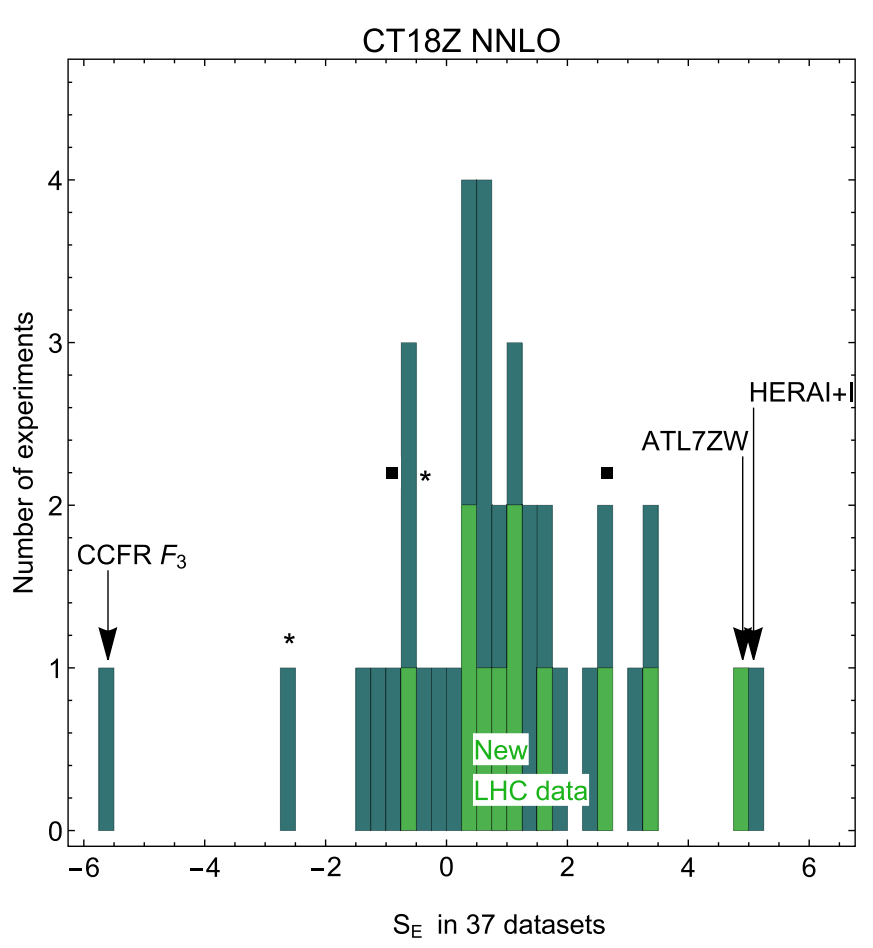

FIG. 59. Analogous to Fig. 4, the effective Gaussian variable $\left(S_{E}\right)$ distribution of all CT18Z datasets. Two squares and two stars indicate the $S_{E}$ values for the $\mathrm{NuTeV}$ dimuon and CCFR dimuon experiments, respectively.

agreement for specific datasets undergoes a number of changes. This can be seen in part by comparing the distribution of $S_{E}$ values obtained for CT18Z in Fig. 59 with what we presented for CT18 in Fig. 4. The inclusion of the ATLAS $7 \mathrm{TeV} W / Z$ data (or, ATL7ZW data, with CTEQ experimental $\mathrm{ID}=248$ ) in $\mathrm{CT} 18 \mathrm{Z}$ causes an upward shift of $S_{E}$ (or $\chi_{E}^{2} / N_{p t, E}$ ) for a number of experiments, notably for the dimuon data from NuTeV (Exp. IDs $=124,125)$ and CCFR (Exp. IDs $=126,127)$, indicating that the ATL7ZW data is in some disagreement with these other datasets.

In Sec. IIC, we pointed out that we release two intermediate fits, CT18A (with addition of ATL7ZW data only) and CT18X (with a specially chosen factorization scale in DIS cross sections). Compared to CT18A and X, the CT18Z PDFs produce maximal changes away from CT18 in the PDFs and their moments, the parton luminosities, and standard-candle predictions: those can be viewed in Figs. 7, 11, 12, 16, 47, and 48.

We will now look into the accumulation of the modifications that led from CT18 to CT18Z NNLO in more detail. The role of the ATL7ZW and CDHSW experiments is reviewed in Sec. A 1. Section A 2 summarizes the key differences between CT18, A, X, and Z PDFs, while the plots of error bands for CT18A and X NNLO and NLO PDFs are included in Supplemental Material [27]. The agreement with the ATL7ZW data is explored in Sec. A 3.
In Sec. A 4, we examine $\chi^{2}$ scans to extract detailed information on the redistribution of constraints on the PDFs inside the CT18Z global fit, as well as on the CT18Z predictions for $\alpha_{s}\left(M_{Z}\right)$ and $m_{c}$.

The physics conclusions presented here have been verified using several independent techniques. Initially, projections of the likely impact of the datasets on the PDFs were obtained by applying the fast Hessian techniques, PDFSENSE $/ L_{2}$ sensitivity [20,21] and EPUMP [25], by starting from theoretical predictions based on the previous CT14 $4_{\text {HERAII }}$ NNLO PDFs [32]. At this stage, we discovered that the EPUMP and $x$ Fitter programs produce very different results when profiling the ATL7ZW data. This discrepancy is addressed in Appendix F. As a part of the fitting itself, we repeated some fits multiple times while either constraining the PDFs at specific values using LM or varying the statistical weights of ATL7ZW and other datasets to explore their mutual consistency within the approach by Collins and Pumplin [192]. All these methods render a coherent physics picture that will be now summarized.

\section{Alterations to datasets and theoretical settings}

\section{a. Modified data selection}

Let us first address some questions arising in the description of two datasets: (i) the recent $7 \mathrm{TeV}$ DrellYan data taken by ATLAS for the rapidity distributions for the inclusive production of $W$ and $Z$ bosons (ATL7ZW, Exp. ID = 248); and (ii) the $F_{2}^{p}, x F_{3}^{p}$ DIS structure function information extracted by CDHSW from $\nu$-Fe data (Exp. IDs $=108,109$ ).

ATLAS $7 \mathrm{TeV}$ inclusive $W / Z$-production data.Regarding case (i), the ATL7ZW dataset is seen as providing important information on the structure of the light-quark nucleon sea, and, in particular, favoring an enhanced value for the strangeness suppression factor, $R_{s}(x, Q)$ of Eq. (10) compared to what has been found in the past global analyses dominated by the DIS data. The sensitivity analysis indicates that, while the correlation cosines of the ATL7ZW measurements with respect to various PDF flavors are quite modest (typically, $|\cos \phi|<0.6$ ), the sheer precision of the ATL7ZW data creates a pronounced pull on all lightantiquark flavors: $\bar{u}$, and $\bar{d}$, and especially $\bar{s}$. For example, the pulls are revealed by the charts showing $L_{2}$ sensitivities of ATL7ZW data to various CT18Z PDF flavors in Fig. 60. The strong positive pull on $s(x, Q)$ revealed by the corresponding negative $S_{L 2, E}$ for $s(x, Q)$ at $x=0.01-0.1$ in Fig. 60 must be compensated in the global fits by the opposing pulls from other experiments. For example, we can compare the Hessian estimates of the pulls on $s(x, Q=$ $2 \mathrm{GeV}$ ) by plotting the respective sensitivities for individual experiments in Fig. 61. It is obvious that the prominent negative pull on $\Delta \chi^{2}$ from ID $=248$ at $x=0.03$ is opposed by the positive pulls from $\mathrm{NuTeV}$ (Exp. IDs $=124,125$ ) 
CT18Z NNLO, ATL7ZW.xF (248), Q=100 GeV

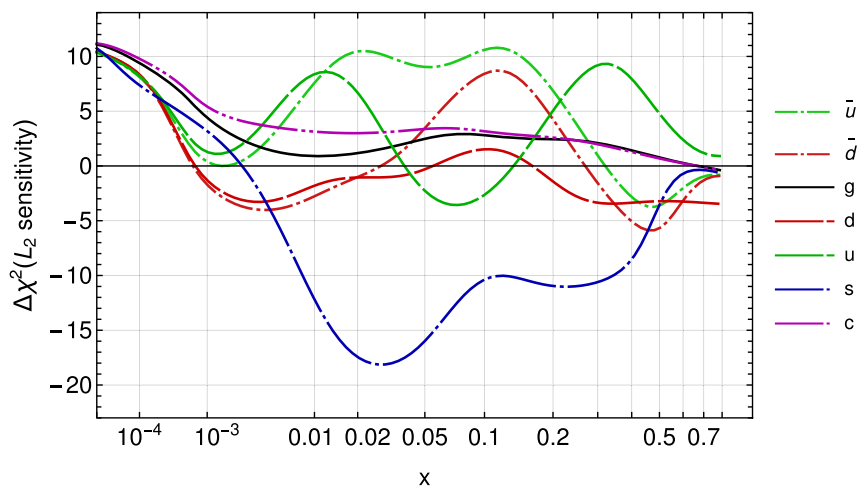

FIG. 60. The $L_{2}$ sensitivity of the ATL7ZW data to the PDFs of several individual parton flavors. The pull on the strangeness distribution, $s(x, Q)$, is particularly large, peaking at $S_{s, L 2} \sim-20$ for $x \sim 0.02-0.05$; although opposing pulls on the $d-, \bar{u}-$, and $\bar{d}$-quark PDFs are also significant in a similar region of $x$.

and CCFR (Exp. IDs $=126,127$ ) dimuon SIDIS, and, also very prominently, the inclusive HERAI + II data $($ Exp. ID = 160). On the other hand, the fixed-target E866 Drell-Yan data on the $p p$ target $($ Exp. ID $=204)$ weakly pulls in the same direction as ATL7ZW, although at smaller $x \approx 0.01$.

While the $L_{2}$ sensitivity estimates contributions to $\chi^{2}$, tensions with the ATL7ZW data are also revealed by other statistical indicators, such as an effective Gaussian variable $S_{E}$ for experiment $E$ that quantifies how the change in $\chi_{E}^{2}$ compares to the respective statistical uncertainty. By this measure, the Hessian updating study [25] based on EPUMP found that including the ATL7ZW data with increasing statistical weights into the CT18 fit strongly increases $S_{E}$ values for the NuTeV $\nu$ SIDIS (Exp. ID = 125), the E866 $\sigma_{p d} /\left(2 \sigma_{p p}\right)$ Drell-Yan data $($ Exp. ID $=203)$, and the CMS $7 \mathrm{TeV}$ electron asymmetry data (Exp. ID = 267), cf. Fig. 25 of [25]. This change is accompanied by modifications in the $s$-quark PDF and $\bar{d} / \bar{u}$ PDF ratio, cf. Fig. 26 of the same reference. Finally, we observe mild suppression of $g(x, Q)$ at $x \gtrsim 10^{-2}$ after including the ATL7ZW dataset.

CDHSW data.-Our LM scans, like the ones presented in Fig. 21 and the $L_{2}$ sensitivity plot in Fig. 26, reveal that the CDHSW measurements of deep inelastic scattering in charged-current neutrino interactions on iron (Exp. IDs = 108 [60], 109 [60]) are sensitive to the gluon distribution at $x>0.2-0.5$ via $Q^{2}$ distributions of their cross sections. At $x<0.4$, the logarithmic slopes of the structure functions

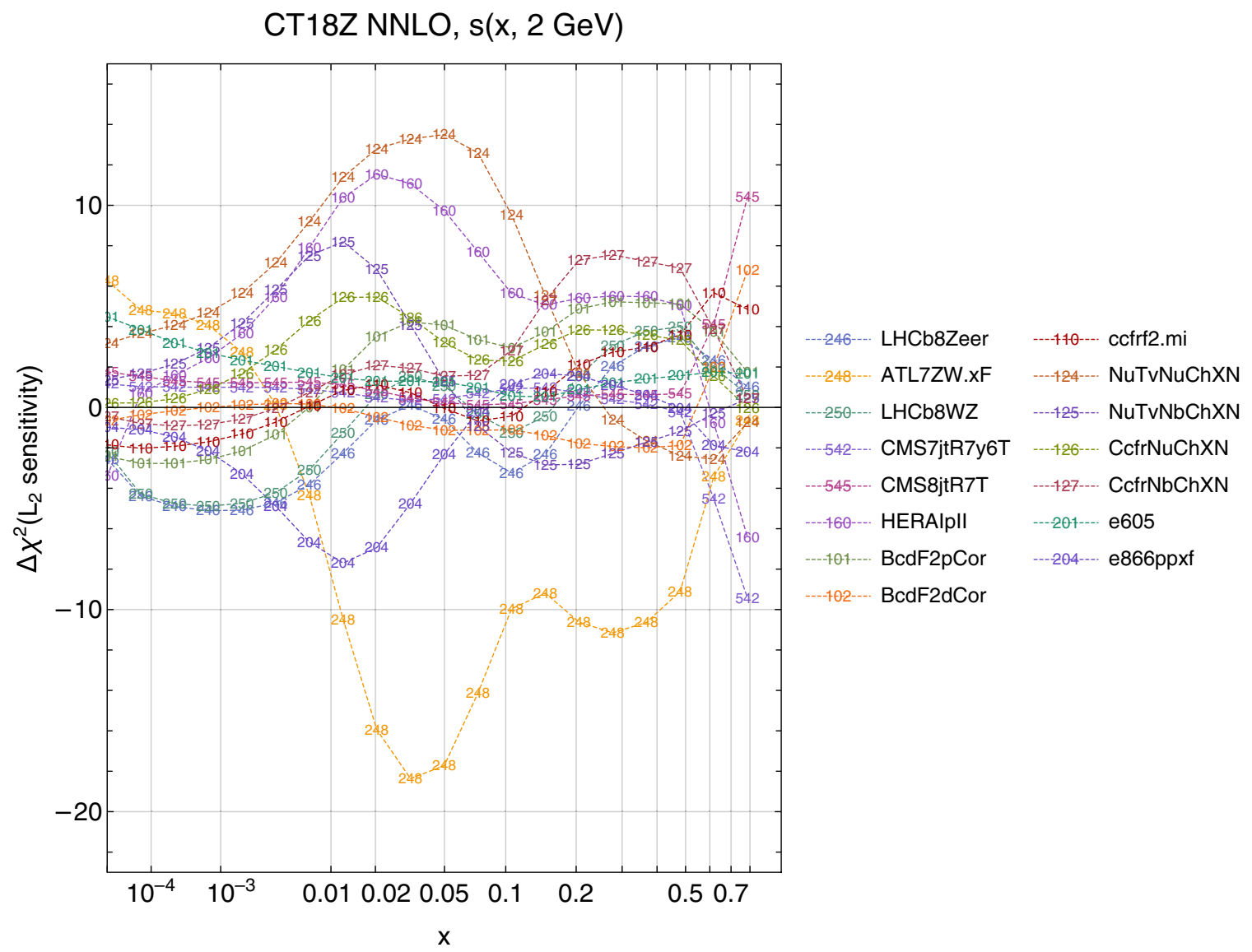

FIG. 61. The $L_{2}$ sensitivity of the data fitted in CT18Z to the $x$-dependent strange PDF at $Q=2$ GeV. Given that the fitted behavior for $R_{S}(x, Q)$ is substantially driven by $s(x, Q)$, the information shown here is complementary to the LM scans in Fig. 68 . 
$F_{2}$ and $x F_{3}$ measured by CDHSW and CCFR are different, cf. Figs. 8.3 and 8.4 in Ref. [92], with CDHSW structure functions preferring a harder $g(x, Q=100 \mathrm{GeV})$ at $x \approx 0.2$, but a softer gluon at $x \gtrsim 0.5$-similarly to the CDF run-2 jet data (Exp. ID = 504). It has been known that unresolved experimental issues may exist in the CDHSW analysis [193], and nuclear corrections may be nonnegligible for the iron target. Thus, one may wonder how the PDFs would change if these two CDHSW datasets are excluded. This question was addressed using EPUMP in Ref. [25], as well as by performing a special fit named "CT18mCDHSW" (i.e., CT18 "minus" CDHSW), in which the two CDHSW datasets were removed from the CT18 global data.

The plots of PDF error bands from the CT18mCDHSW NNLO fit, included in the Supplemental material [27], show that removing CDHSW data leads to a slight reduction in the gluon PDF at $x=0.1-0.5$, combined with a slight increase in the gluon PDF uncertainty, and compensating increases in the $u$ and $d$ PDFs in the same $x$ region.

\section{b. The $x_{B}$-dependent scale and modified global fits}

Another point of potential concern is the residual dependence on QCD cross sections on the renormalization and factorization scales, which we find to be non-negligible in some experiments, compared to the latest experimental uncertainties, even when NNLO theoretical expressions for QCD cross sections are used. In particular, by evaluating the NNLO DIS cross sections at a carefully chosen factorization scale $\mu_{F, x}$ dependent on Bjorken $x_{B}$ in Table III, we moderately improve agreement with HERA DIS cross sections: the respective $\chi^{2}$ improves by 40 units $\left[\chi^{2}(\mathrm{CT} 18)=1408, \chi^{2}(\mathrm{CT} 18 \mathrm{Z})=1378\right.$ for $N_{p t}=1120$ data points], and it can be improved by another 30 units by increasing the statistical weight of the inclusive HERA data to 10 as in the right Fig. 3. With this scale choice, we also obtain larger PDFs for the gluon and strangeness PDFs at momentum fractions $x$ below 0.05 , cf. left Fig. 3. At the same time, $g(x, Q)$ and $s(x, Q)$ are reduced from $x=0.2$ to $0.4-0.5$ to preserve the sum rules.

Therefore, three modifications in the global fitting framework-using the $\mu_{F, x}$ scale in DIS, excluding the CDHSW datasets, and adding the ATLAS7ZW data-add up to suppress the gluon PDF at $0.005 \lesssim x \lesssim 0.3$, across most of the interval of $x$ relevant for the LHC Higgs production via $g g$ fusion. Their combination also produces a substantial increase of $s(x, Q)$ at all $x$.

As discussed in Sec. II C, this combination is adopted to produce the CT18Z NNLO PDFs. The intermediate PDFs, CT18A and CT18X, implement only the ATL7ZW data in the $66<Q<116 \mathrm{GeV}$ region (34 data points) or only the DIS scale $\mu_{F, x}$, as indicated in Table III. [The lowluminosity $\left(35 \mathrm{pb}^{-1}\right)$ sample of the ATLAS $7 \mathrm{TeV} W^{ \pm}$ and $Z$ cross section data (Exp. ID $=268$ ) is removed from the CT18A and Z fits to avoid double counting.]

\section{Comparisons between the four PDF ensembles}

Supplemental Material [27] includes a series of figures comparing the NNLO and NLO PDF uncertainty bands for CT18, A, X, and Z PDF flavors. The main characteristics of these comparisons can be distilled as follows:

(1) $g(x, Q)$ : By comparing the PDF uncertainty bands for CT18, CT18A, and CT18Z, on one hand, and CT18, CT18X, and CT18Z, on the other hand, it is clear the bulk of the variation of CT18Z away from CT18 is due to the modified DIS scale choice, $\mu_{F, x}$, with only weaker changes resulting from the interplay of the removal of CDHSW and inclusion of the ATL7ZW measurements. The deviations from CT18 are generally smaller at NLO, with the exception of the very low- $x$ region, where CT18Z and CT18X NLO ratios to CT18 are $\sim 50 \%$ larger.

(2) $d(x, Q)$ : In contrast to $g(x, Q)$, the sensitivity of the Drell-Yan process to $d(x, Q)$ enhances the difference between the CT18 and CT18A NNLO results for this flavor, realized as a mild, $\sim 1 \%$ suppression of the central CT18A distribution for $d(x, Q)$ relative to CT18 about $x \sim 10^{-3}$, with a few-percent reduction in the accompanying PDF uncertainty. The CT18Z fit in this region is pulled in the opposing direction, being enhanced by $\lesssim 2 \%$ with a comparable uncertainty for $x<0.1$. In the high- $x$ region, $x \gtrsim 0.2$, the effect of fitting the ATL7ZW data alone in CT18A NNLO boils down to a small shift in the $d(x, Q)$ uncertainty. CT18Z, in contrast, is suppressed relative to CT18 at higher values of $x$. The qualitative impact of fitting $d(x, Q)$ at NLO, as opposed to NNLO, is fairly weak, being felt mostly at lower $x \lesssim 10^{-3}$.

(3) $u(x, Q)$ and $\bar{u}(x, Q):$ Here, the remarkable property is the extent to which the deviations of CT18Z NNLO away from CT18 NNLO are driven by the modifications in CT18X, as attested by the very close agreement between the central PDFs and uncertainties for CT18Z and X. The weak suppression of $\bar{u}(x, Q)$ in CT18A NNLO is almost entirely nulled once the DISscale choice found in CT18X is implemented in CT18Z on top of the ATL7ZW measurements.

(4) $s(x, Q)$ and $R_{s}(x, Q)$ : We have noted already that the introduction of the ATL7ZW data in CT18A/Z NNLO fits leads to a demonstrable enhancement of $s(x, Q)$ over CT18, while the scale choice in CT18X mildly suppresses $s(x, Q)$ at $0.01 \lesssim x \lesssim 0.5$ and enhances it at $x \lesssim 0.01$ and $x \gtrsim 0.5$. The strangeness suppression ratio, $R_{s}(x, Q)$, is driven for the most part by the patterns observed for $s(x, Q)$ itself.

\section{A closer look at the description of ATLAS $7 \mathrm{~W} / \mathrm{Z}$ data}

\section{a. Goodness of fit to ATL7ZW data in CT18A(Z)}

Turning now to the overall description of the ATL7ZW measurement, responsible for the strong modifications of 
the nucleon strangeness in CT18A/Z NNLO, we find a significant improvement in $\chi^{2}$ for these data once they are actually fitted. Namely, CT18 NNLO produces a very large $\chi_{E}^{2} / N_{p t, E}=8.4\left(S_{E}=13.7\right)$, which diminishes substantially to $\chi_{E}^{2} / N_{p t, E}=2.6\left(S_{E}=4.8\right)$ in CT18Z NNLO, as we reported in Table II. We note that the corresponding values for CT18A NNLO, which deviates from the settings of CT18 NNLO only in the implementation of the ATL7ZW data, are only a very slight improvement over CT18Z, $\chi_{E}^{2} / N_{p t, E}=2.6\left(S_{E}=4.7\right)$. We also remind the reader that the $\mathrm{CT} 18 \mathrm{~A} / \mathrm{Z}$ fits include only 34 ATL7ZW data points in the resonance region $(66<Q<116 \mathrm{GeV})$, with the rest of 61 points of the published set being less precise and contributing mostly to the reduction of $\chi_{E}^{2} / N_{p t, E}$ without really improving the PDF constraints, while also enhancing the dependence of the PDFs on NLO EW corrections in the off-resonance regions.

In comparison, the ATLAS group themselves obtained $\chi_{E}^{2} / N_{p t, E}=108 / 61$ in the ATLAS-epWZ16 fit [39] of the $W / Z$ and combined HERA I + II data [30]. For CT14 PDFs, they obtained $\chi_{E}^{2} / N_{p t, E}=103 / 61=1.69$ after profiling the CT14 PDFs with the $W / Z$ data in xFitter. Their fitted strangeness fraction is $R_{s}=(s+\bar{s}) /(\bar{u}+\bar{d})=$ $1.13 \pm 0.08$ at $x=0.023$; i.e., it is significantly larger than what we obtained in the CT18A(Z) fits.

We confirm these findings when including only the ATL7ZW and HERA I + II data in the CT analysis. In fact, we get a better $\chi^{2} / N_{p t, E} \approx 1.4$ and even larger $R_{s}$, due to the more flexible CT parametrization, if the ATL7ZW data are included with an elevated statistical weight.

However, we view this outcome as problematic on the grounds that

(1) The above xFitter profiling analysis strongly deemphasizes the experiments that show tension with the ATL7ZW data, as explained in Appendix F;

(2) Our LM scans like the ones presented in Sec. A 4 reveal that CT fits, with their increased flexibility compared to HERAPDF fits, become unstable or have multiple minima when $R_{s}(x, Q)$ is forced to be close to 1 at $x=0.01-0.1$, as preferred by the ATL7ZW dataset; the instability may reflect the still weak capability of data to discriminate between the $\bar{s}, \bar{d}$, and $\bar{u}$ contributions.

Other PDF fitting groups have also investigated the ATL7ZW data. Table IX summarizes the values of $\chi_{E}^{2} / N_{p t, E}$ for the ATL7ZW data obtained in CT18A, Z, ATLAS-epWZ16, MMHT (2019), and NNPDF3.1 NNLO fits. For the CT18A/Z fits, we quote the $68 \%$ C.L. uncertainties on $\chi_{\mathrm{ATL} Z \mathrm{ZW}}^{2}$. We find them in Sec. A 4 a from the LM scan on the weight of $\chi_{\text {ATL7ZW }}^{2}$ shown in the left panel of Fig. 67. The uncertainties are equal to the differences of $\chi_{\text {ATL7ZW }}^{2}$ from the value of $\chi_{\text {ATL7ZW }}^{2}$ at the best global fit, when the total $\chi^{2}$ (solid black line in the left
TABLE IX. The comparison of the $\chi^{2}$ values for ATLAS $7 \mathrm{TeV}$ $W / Z$ data among the QCD analysis from different groups. For CT18A/Z PDFs, we show the $68 \%$ C.L. uncertainties obtained by a Lagrange multiplier scan on the $\chi^{2}$ weight of the ATLAS $W / Z$ dataset, as illustrated in Fig. 67 (left).

\begin{tabular}{lcccc}
\hline \hline PDF & $N_{p t, E}$ & $\chi_{E}^{2}$ & $\chi_{E}^{2} / N_{p t, E}$ & Ref. \\
\hline CT18A & 34 & $88_{-28}^{+68}$ & $2.58_{-0.84}^{+2.01}$ & \\
CT18Z & 34 & $89_{-31}^{+65}$ & $2.61_{-0.91}^{+1.90}$ & \\
ATLAS-epWZ16 & 61 & 108 & 1.77 & {$[39]$} \\
MMHT (2019) & 61 & 106.8 & 1.76 & {$[19]$} \\
NNPDF3.1 & 34 & 73 & 2.14 & {$[3]$} \\
\hline \hline
\end{tabular}

panel of Fig. 67) is increased by one standard deviation (36 $\chi^{2}$ units).

The respective $\chi_{\mathrm{ATL} 7 \mathrm{ZW}}^{2}$ values quoted in Table IX are well within the $68 \%$ uncertainties of CT18A/Z, with the nominal CT18A/Z values for $\chi_{\mathrm{ATL} \text { 7ZW }}^{2}$ being on the high side, but not significantly. The differences in $\chi_{\text {ATL7ZW }}^{2}$ can be traced primarily to the magnitude of $s(x, Q)$ at moderate $x$, which is lower (within the uncertainties) in CT18A/Z NNLO than in some other fits.

The ABM analysis [194] has emphasized tensions between ATL7ZW, NuTeV, and NOMAD datasets, as well as strong dependence of the preferred $R_{s}(x, Q)$ enhancement on the flexibility of the strangeness parametrization. The unpublished 2019 MMHT analysis [19] obtains $\chi_{E}^{2} / N_{p t, E}=1.76$ for 61 ATL7ZW data points by (a) including NNLO quark-mass corrections for inclusive chargedcurrent (SI)DIS cross sections [122,178], which slightly improve agreement between the DIS and ATL7ZW datasets, cf. Sec. V B 4; and (b) using flexible six-parameter parametrizations for $d$ and $s$ quarks. The 2019 MMHT analysis reports a significant enhancement in $s(x, Q)$ above that in MMHT14 for $x>10^{-3}$, with a corresponding uncertainty reduction.

While NNPDF did not actively fit ATL7ZW in NNPDF3.0, which obtained $\chi_{E}^{2} / N_{p t, E}=8.44$, these data were implemented in NNPDF3.1 [3], resulting in $\chi_{E}^{2} / N_{p t, E}=$ 2.14 - close to CT18A/Z NNLO. NNPDF3.1 observed an enhancement in the fitted strange PDF that is similar to that in CT18A NNLO for $x<0.1$. Figure 62 plots the ratios of the CT18A and NNPDF3.1 NNLO strangeness PDFs at $Q=$ $100 \mathrm{GeV}$ to those in CT18 (here, a baseline which did not include ATL7ZW). In CT18A, we observe a $10 \%$ excess above CT18 in the region $x \gtrsim 0.02$ where the ATL7ZW data have the strongest pull. Especially in this region of $x$, the larger $s$ PDF found in CT18A is closely reflected by the strangeness fitted in NNPDF3.1.

The agreement with the ATL7ZW dataset is impacted by the choice of the NNLO code for Drell-Yan pair production, as discussed at the end of Appendix F, and also by the choice of the QCD scales, set equal to $\mu_{R, F}=Q$ in the CT18A/Z fits and to $\mu_{R, F}=Q / 2$ in MMHT (2019), where 


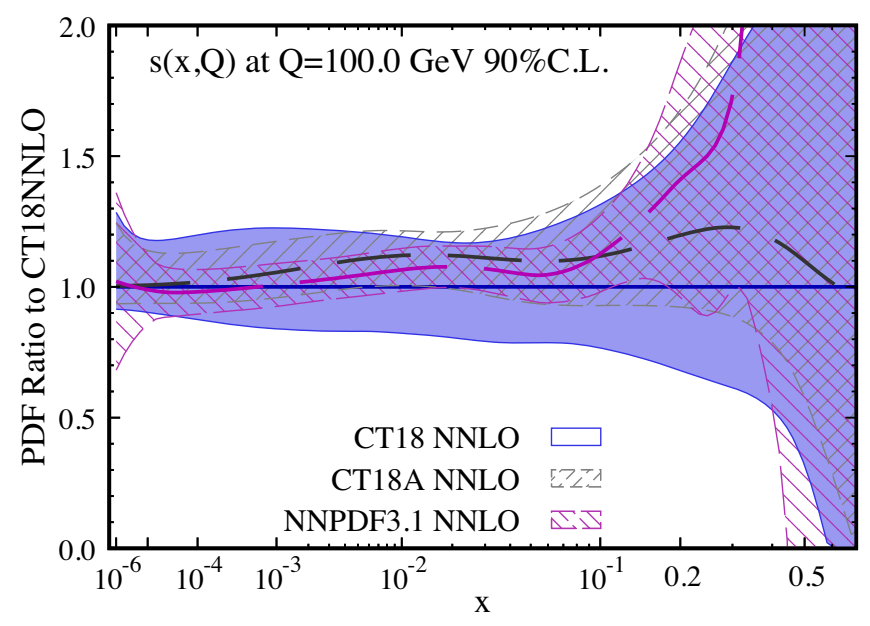

FIG. 62. A comparison of the fitted strangeness PDF, $s(x, Q)$, at $Q=100 \mathrm{GeV}$ as obtained with CT18 (solid violet line) as well as with CT18A (short-dashed gray line) and NNPDF3.1 (longdashed magenta line).

$Q=M_{W}\left(M_{\ell \bar{\ell}}\right)$ in $W(Z)$ boson production. Inclusion of associated $W$-boson and charm-jet $(W+c)$ production data in the fit, like the dataset by $\mathrm{CMS}$ at $7 \mathrm{TeV}$ [111] included by NNPDF3.1, tends to create an extra upward pull on $s(x, Q)$. We do not include $W+c$ measurements in CT18A(Z) yet because the full NNLO calculation is still unavailable. Neither are the NNLO massive heavy-quark contributions for differential cross sections of SIDIS dimuon production, needed to compute the detector acceptance when the fitted $\mathrm{CCFR} / \mathrm{NuTeV}$ cross sections are reconstructed.

\section{b. Comparisons to rapidity distributions}

Figures 63-65 show the theory-to-data comparisons for CT18A/Z predictions against the ATL7ZW dataset, the distributions of shifted residuals in the final-state (pseudo) rapidity in three individual channels, and the respective cumulative histograms of the best-fit shifted residuals $r_{i}$ and nuisance parameters $\bar{\lambda}_{\alpha}$.

In terms of the descriptions of the individual data points, the figures indicate that $\mathrm{CT} 18 \mathrm{~A} / \mathrm{Z}$ predictions describe $W^{+}$production well, while they show elevated differences with $W^{-}$production across the whole range of lepton pseudorapidities, as well with $Z$ production in the bins with the average $Z$-boson rapidity of 0.9 and 1.3.

Description of these data also require shifts of five nuisance parameters $\bar{\lambda}_{\alpha}$, labeled as $113,129,72,125$, and 128 in the ATLAS dataset, by $\approx \pm 2$ standard deviations. (These parameters receive a mix of contributions from various systematic sources and do not have a certain physics interpretation.)

A variation on the $L_{2}$ sensitivity technique allows us to demonstrate that variations in these parameters are strongly linked to changes in the strangeness ratio $R_{s}$ in the $x$ region probed by the ATL7ZW measurement. In the previous figures, the $L_{2}$ sensitivity approach explored the connection between PDF variations and $\Delta \chi_{E}^{2}$ for individual experiments; but we can also compute the $L_{2}$ correlation between the PDFs and contribution to $\chi^{2}$ from an individual nuisance parameter, $\Delta \bar{\lambda}_{\alpha}^{2}(a)$ for $\alpha=1, \ldots, N_{\lambda}$, contributing

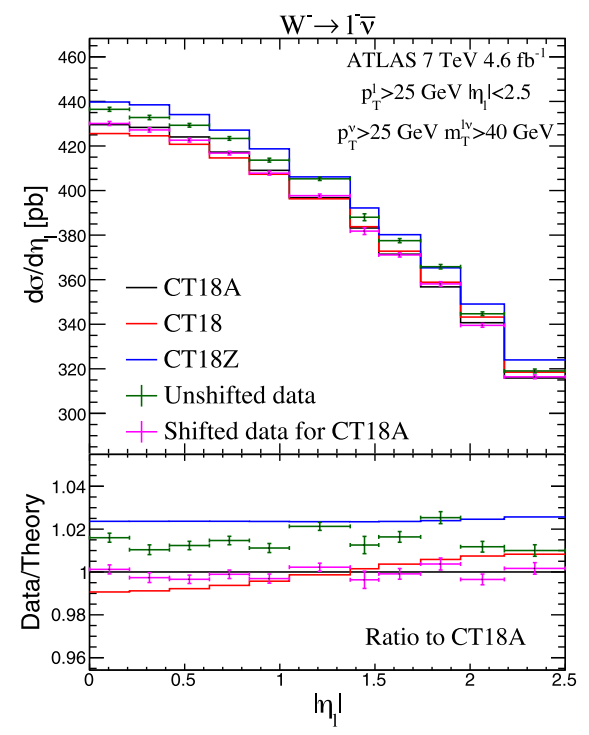

(a) $W^{-}$production

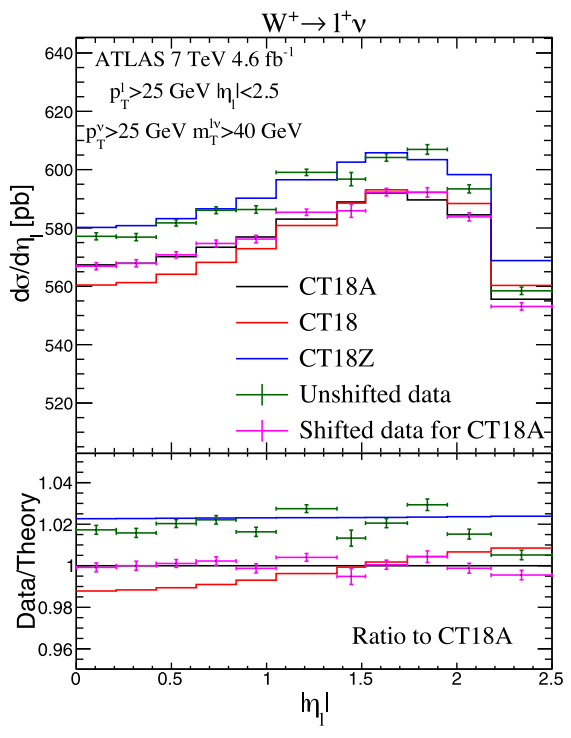

(b) $W^{+}$production

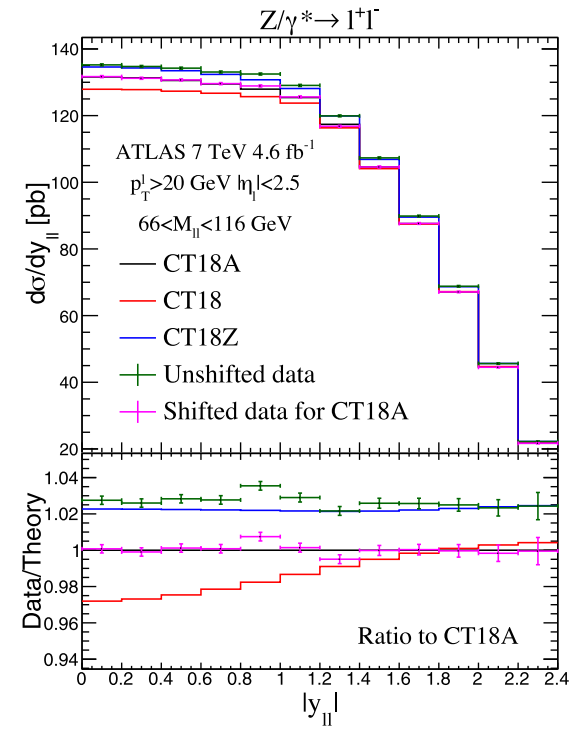

(c) $Z$ production

FIG. 63. (a) A comparison of theoretical predictions for the ATL7ZW data based on CT18 and CT18A/Z NNLO. The shifted data (magenta crosses) are computed based on CT18A NNLO. The upper panels give rapidity distributions of the differential cross sections in $\left|\eta_{l}\right|$ (for the charge-current processes) or $\left|y_{\ell \bar{\ell}}\right|$ (for neutral current), while the lower insets show the ratios of the data and theory, normalized to CT18A theory. 


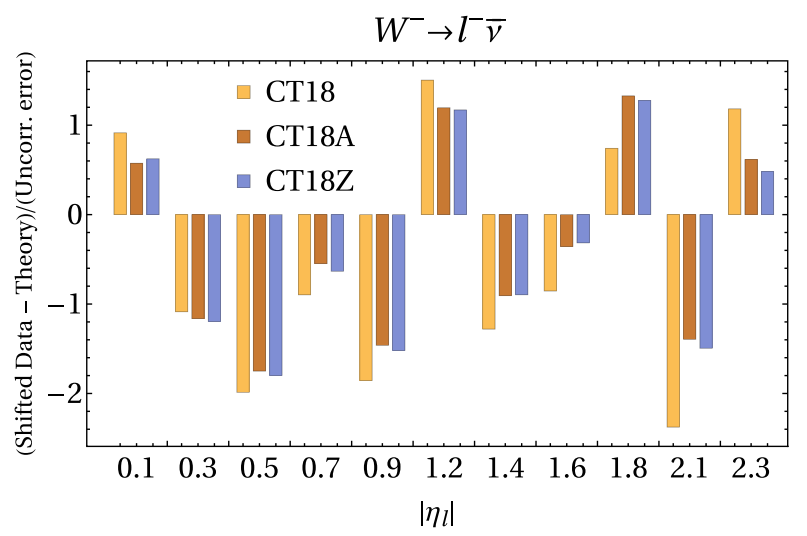

(a) $W^{-}$production

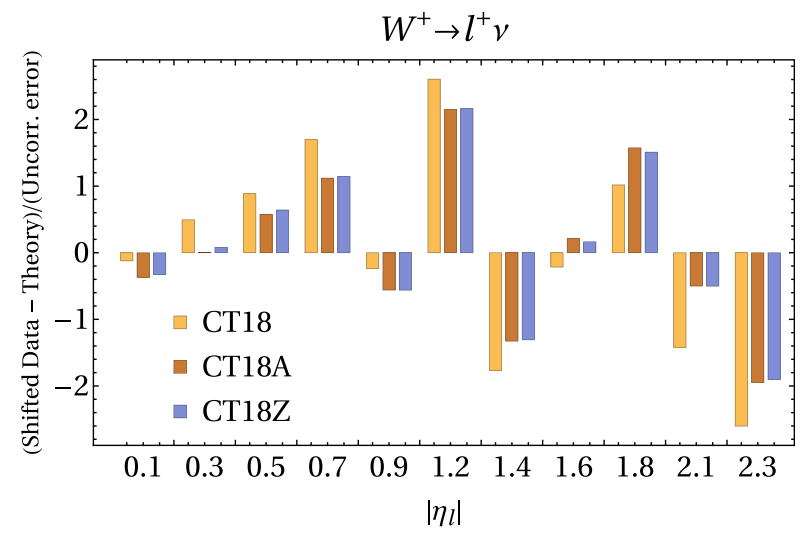

(b) $W^{+}$production

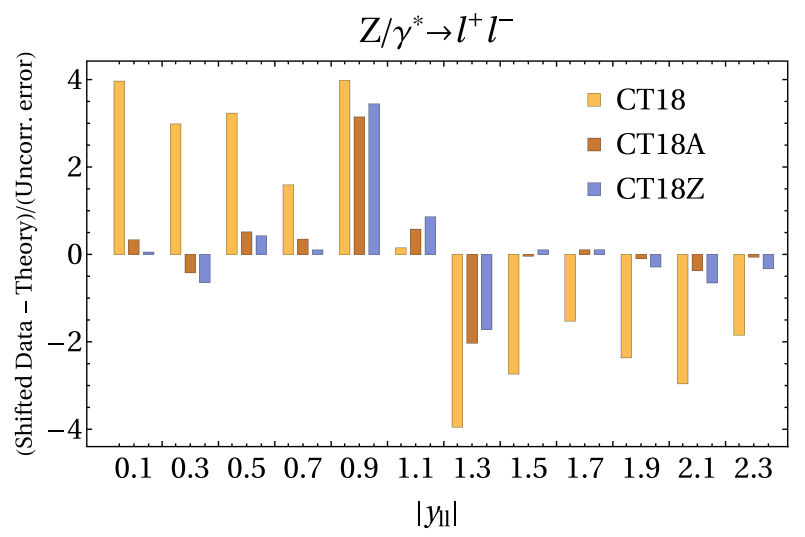

(c) $Z$ production

FIG. 64. Bin-by-bin shifted Theory-Data residuals computed based on CT18(A/Z) for each of the three ATL7ZW processes shown in Fig. 63.

to $\Delta \chi_{E}^{2}(a)$ according to Eq. (2) for arbitrary $a \approx a_{0}$. In Fig. 66, we demonstrate this for the ATL7ZW data, showing the pulls of the ATL7WZ correlated systematic uncertainties on $R_{s}$ at $Q=100 \mathrm{GeV}$ in CT18A. While most nuisance parameter shifts stay within $\left|\Delta \lambda_{\alpha}^{2}\right| \sim 1-2$ units, a small collection of $\bar{\lambda}_{\alpha}$ are strongly sensitive to the fitted $R_{s}$ at various $x$, notably parameters $113,72,120$, and
129 , of which some very nearly approach (parameters 72 and 129) or exceed (parameters 113 and 120) the bound of $\left|\Delta \lambda_{\alpha}^{2}\right|<2$.

While we do not have information to reveal the specific causes driving these systematic sources, it is clear that some have profound effect on the preferred $R_{s}$ behavior in the intervals of $x$ that can be read off Fig. 66.

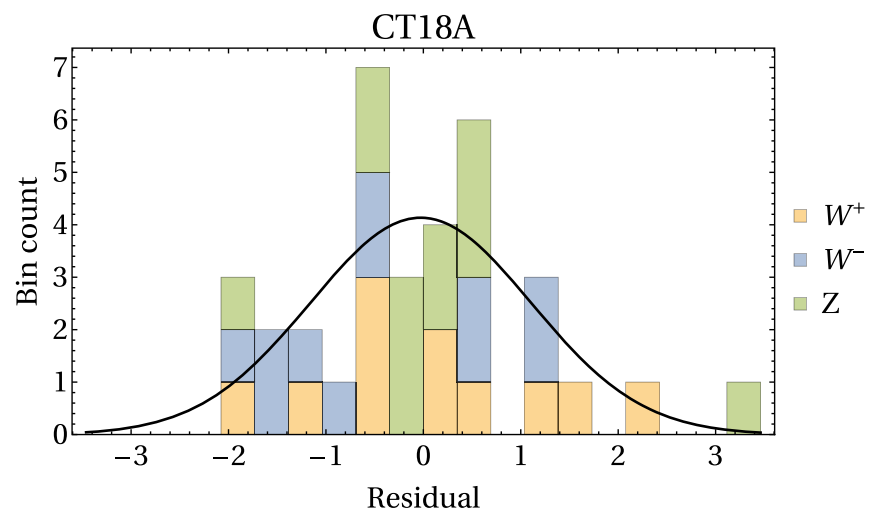

(a)

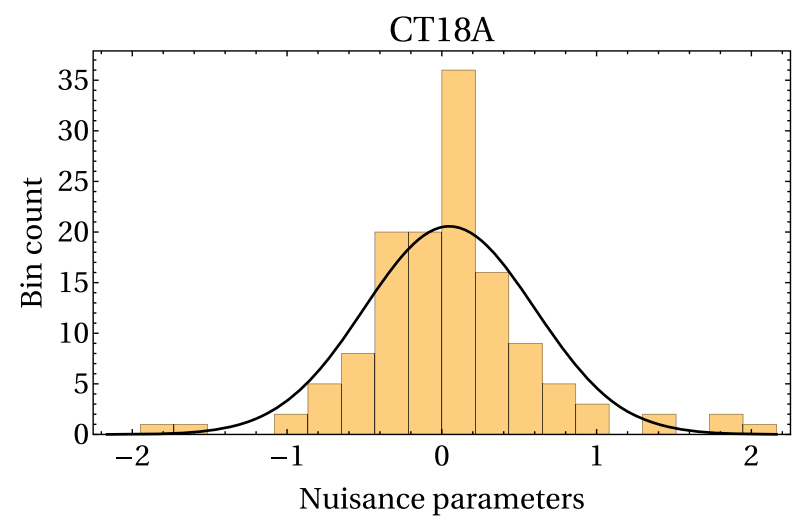

(b)

FIG. 65. Histogram of (a) the shifted residuals and (b) nuisance parameters for the ATL7ZW data obtained for CT18A NNLO. 


\section{CT18A NNLO, ATL7ZW.xF, 2s/( $\bar{u}+\bar{d})(x, 100 \mathrm{GeV})$}

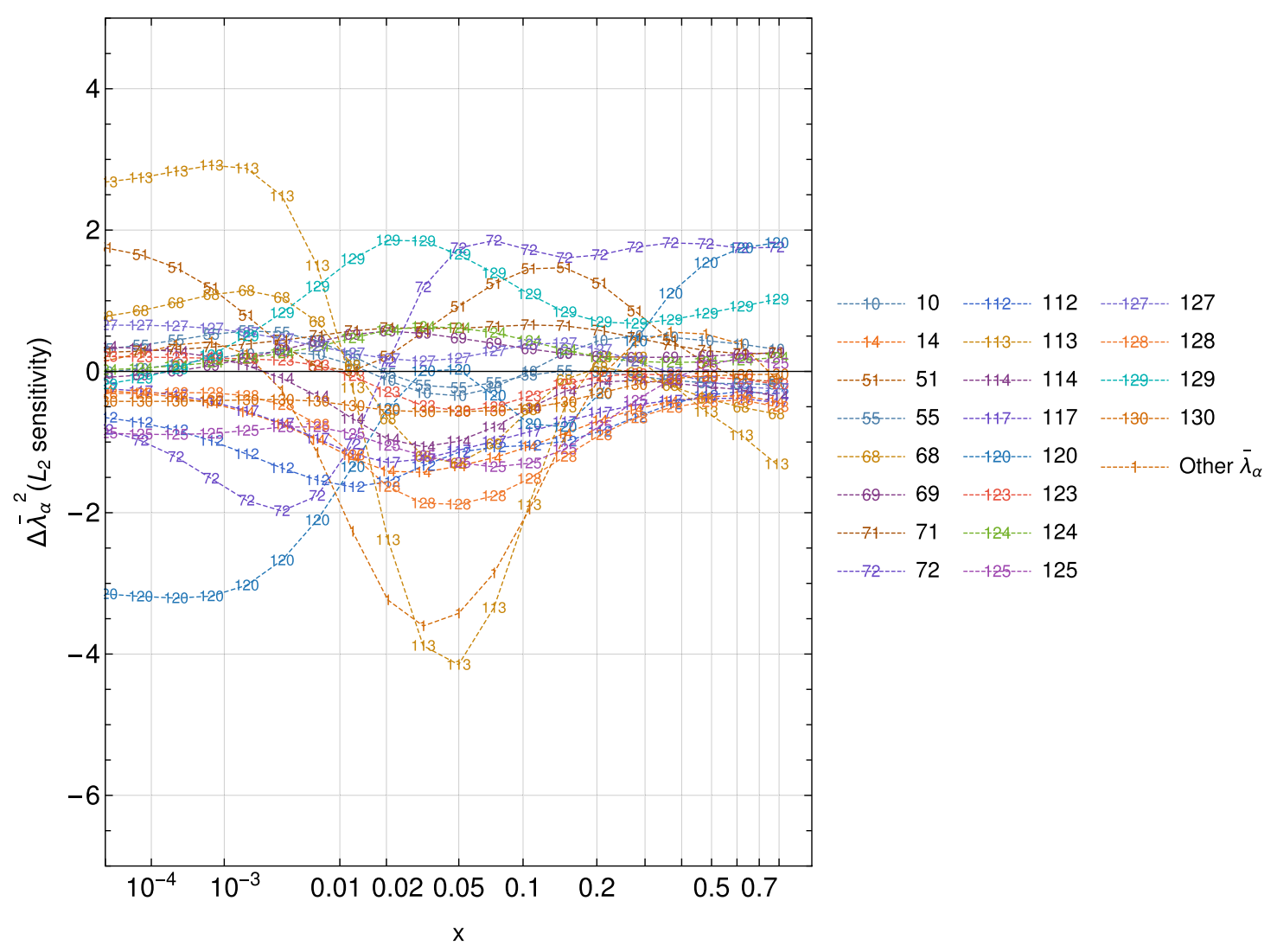

FIG. 66. A plot, analogous to the $L_{2}$ sensitivity plots given above, showing the pulls of the correlated systematic uncertainties of the ATLAS $W / Z$ data on $R_{s}(x, Q=100 \mathrm{GeV})$ in CT18A NNLO, as represented by shifts in the associated squared nuisance parameters, $\Delta \lambda_{\alpha}^{2}$.

\section{c. Minisummary}

The precision of the ATL7ZW data results in a strong impact on the strange quark distribution when included in the CT18 global PDF fits. The presence of the data in the fit (either CT18A or CT18Z) leads to a greatly improved $\chi^{2}$ for that dataset, compared to a straight evaluation with the CT18 PDFs, but still significantly above one per degree of freedom. The effective Gaussian variable for this dataset is also large, comparable only to that for HERA I + II. Overall, tensions with the other CT18 global data lead to a less consistent fit, as quantified by the strong goodnessof-fit criteria [37], and have resulted in the ATL7ZW data being included in the CT18A/Z PDFs, but not in the CT18 PDF. Additional precise LHC data may further resolve the strangeness issue. In the meantime, a full exploration of the strangeness uncertainty will require the use of the CT18A or CT18Z PDF sets.

\section{Scans on PDFs, $\alpha_{s}$, and $m_{c}$ in CT18Z}

We conclude this Appendix with a few results from Lagrange multiplier scans, the powerful technique applied at the end of the global analysis cycle to obtain a close look at the exact probability distributions that cannot be gleaned from the fast, but approximate, Hessian studies. The results presented here for CT18Z NNLO complement an analogous discussion for CT18 presented in Sec. VA. By examining the $\Delta \chi^{2}$ values that are returned from the full fit rather than from the fast linearized approximations, we discover subtle features such as the tensions among experiments or instability/multiple solutions of the fit for some PDF combinations.

\section{a. Scans with varied statistical weights of ATLAS $7 \mathrm{TeV} W / Z$ and NuTeV data}

The evident tension between the ATL7ZW data and other experiments can be examined in terms of the variation of $\chi_{E}^{2}$ for a number of most sensitive experiments, when the weight of the ATL7ZW data is varied within the CT18Z NNLO fit [192]. The results of this are shown in the left panel of Fig. 67, in which the weight of the ATL7ZW data is continuously tuned from $w=0.1$ (sharply deemphasizing this information, shifting $\chi_{E}^{2}$ by $\Delta \chi_{E}^{2} \sim+80$ ) to $w=20$ (strongly over weighting the ATLAS data, leading to a $\Delta \chi_{E}^{2} \sim-40$ improvement in their 

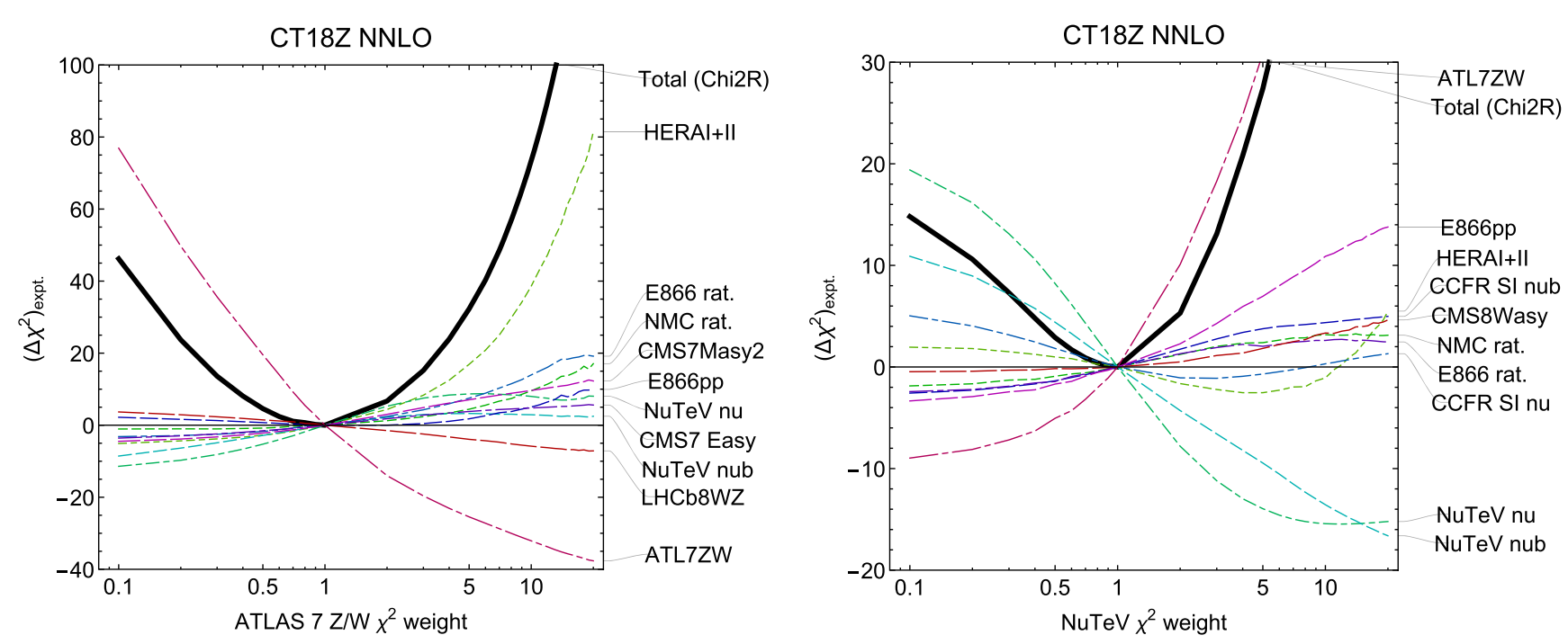

FIG. 67. Left panel: the change in total $\chi^{2}, \Delta \chi^{2}$, of the leading datasets included in the CT18Z NNLO fit when varying the weight of the ATL7ZW data away from weight $w=1$ as in the default fit. Right panel: the analogous plot for the $\mathrm{NuTeV} \nu, \bar{\nu} \operatorname{SIDIS}$ dimuon data $($ Exp. IDs $=124,125)$.

description in CT18Z). The uncertainties in $\Delta \chi_{E}^{2}$ for the ATL7ZW data reported in Table IX correspond to an increase in the total $\chi^{2}$ of 36 .

The figure also shows the curves for the experiments with the largest variations in their $\Delta \chi_{E}^{2}$ when the $\chi^{2}$ weight of the ATL7ZW dataset is changed. A notable feature to observe in the left inset is that it is the combined HERAI + II inclusive DIS dataset (Exp. ID = 160), rather than other experiments, that mostly opposes including the ATL7ZW dataset with a weight of 5-10. With the exception of the LHCb $8 \mathrm{TeV} W / Z$ data (250), which is described better when the weight of ATL7ZW is increased, the rest of the plotted experiments oppose such increase. These experiments include E866 (203) and NMC (104) $p / d$ ratio, $\mathrm{NuTeV}$ dimuon production $(124,125)$, and CMS $7 \mathrm{TeV}$ $\mu$ - and $e$-asymmetries data (266 and 267).

As the ATLAS data are deemphasized $(w \sim 0.1)$, the descriptions of the NuTeV SIDIS dimuon-production datasets (Exp. IDs $=124,125)$ are most improved, further suggesting some tension.

These observations are consistent with the companion scan shown in the right inset, which similarly plots the $\Delta \chi^{2}$ variations of the experiments most responsive to changing the weight of the NuTeV dimuon data. Especially considering $N_{p t}$ for the plotted experiments, the heavy overweighing of the $\mathrm{NuTeV}$ data leads to a very rapid deterioration of $\chi^{2}$ for the ATL7ZW points, which in fact worsens more quickly than the full CT18Z global analysis as the NuTeV weight is increased. In fact, the $\chi_{E}^{2}$ for the inclusive HERA (160) and CCFR neutrino dimuon production (126) mildly improves when the NuTeV weight is increased to about 5. On the other hand, E866 pp (204), CCFR antineutrino dimuon (127), CMS $8 \mathrm{TeV}$ charge asymmetry (249), and NMC $p d$ ratio (104) oppose such increase.

\section{b. LM scans on the strangeness ratio $\boldsymbol{R}_{s}$ for CT18Z}

Lagrange multiplier scans on the strangeness ratio $R_{s}(x, Q)$, shown for CT18Z NNLO in Fig. 68 at $Q=$ $1.5 \mathrm{GeV}$ and two representative momentum fractions, $x=$ 0.023 (in the left panel) and $x=0.1$ (in the right), reveal several important features that are also observed in the other LM scans we have performed.

Both panels of Fig. 68 reveal the opposing preferences of, e.g., the HERA DIS and NuTeV/CCFR SIDIS sets for a smaller value of $R_{s}$, peaked more toward $R_{s} \sim 0.5$ at both values of $x$, and the positive pull of the ATL7ZW data, supported by weaker pulls of E866 $p d$ ratio (203), BCDMS $F_{2}^{d}$ (102), and especially $F_{2}$ CCFR neutrino (110) data, which is very pronounced at $x=0.1$.

When $R_{s}$ is forced to take values above $0.7-0.8$, the $\chi^{2}$ starts to fluctuate irregularly in both scans and fails to converge in many fits as $R_{s}$ is pushed to even higher values.

In reflection of the above strong trade-off between the pulls of the DIS and ATL7ZW datasets, we observe that the Hessian uncertainty for $R_{S}$, based either on the CT two-tier tolerance or dynamic tolerance used by MMHT, does not fully capture the true $\chi^{2}$ behavior revealed by the LM scans in Fig. 68. We remind the reader that CTEQ-TEA Hessian eigenvector PDFs are constructed using a two-tier prescription $[38,42]$ that prevents either too large increase in the global $\chi^{2}$ or too large increases in $\chi_{E}^{2}$ values of individual experiments. Figure 69 illustrates several estimates for $68 \%$ C.L. uncertainty intervals for $R_{s}(0.023,1.5 \mathrm{GeV})$ in the CT18A NNLO fit. (The findings are similar for the CT18Z NNLO fit.) The upper estimate is based on the CT18A Hessian eigenvector set, with the 68\% C.L. estimate obtained by dividing the $90 \%$ C.L. asymmetric uncertainty by 1.645 . Right below the CT18A Hessian estimate, we 


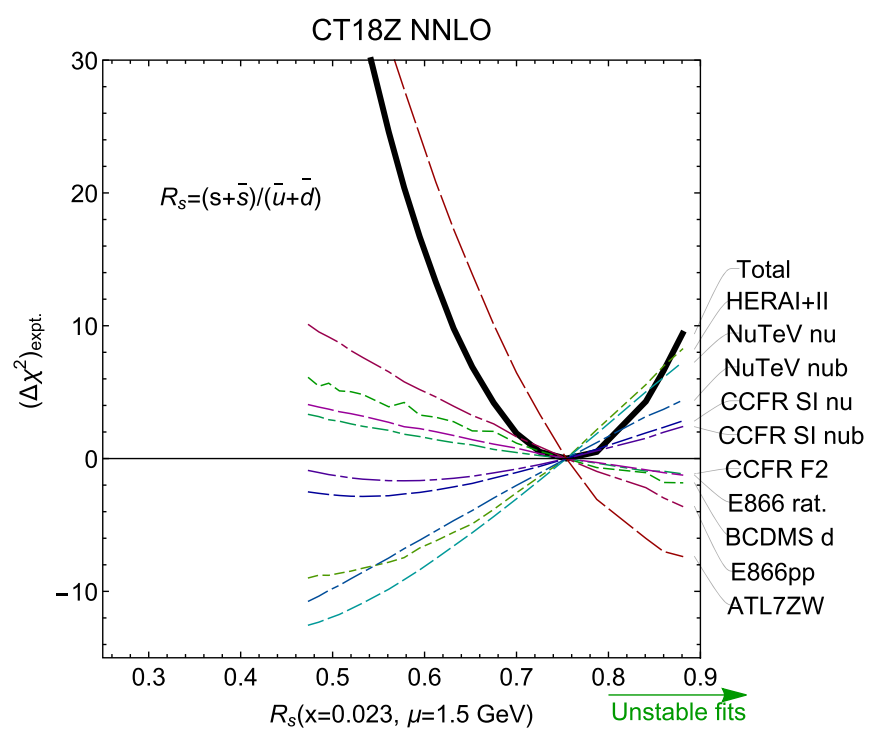

(a)

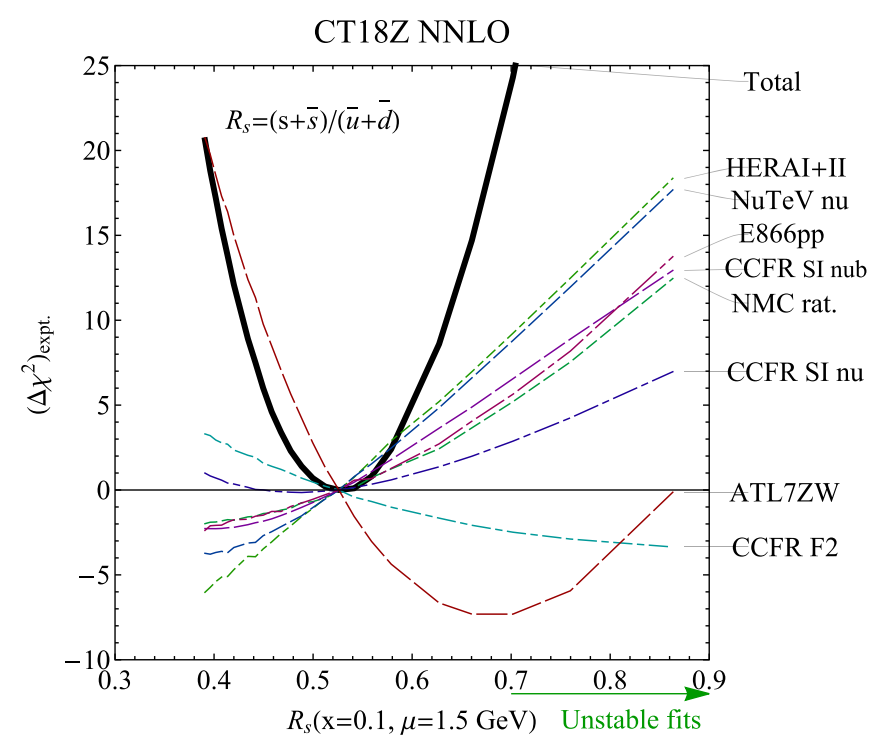

(b)

FIG. 68. The Lagrange multiplier scan of $R_{s}$ at $Q=1.5 \mathrm{GeV}, x=0.023$, and $x=0.1$ for the (a),(b) CT18Z fit, analogous to Fig. 24 for CT18.

show the interval corresponding to $\Delta \chi_{\text {total }}^{2}=10$ in the $R_{s}$ scan in the left Fig. 68. We see that, while the CT Hessian uncertainty nominally corresponds to $\Delta \chi_{\text {total }}^{2} \approx 36$, in fact its uncertainty interval is comparable to that for the true $\Delta \chi_{\text {total }}^{2} \approx 10$ from the LM scan. The true uncertainty is wider than the Hessian estimate suggests.

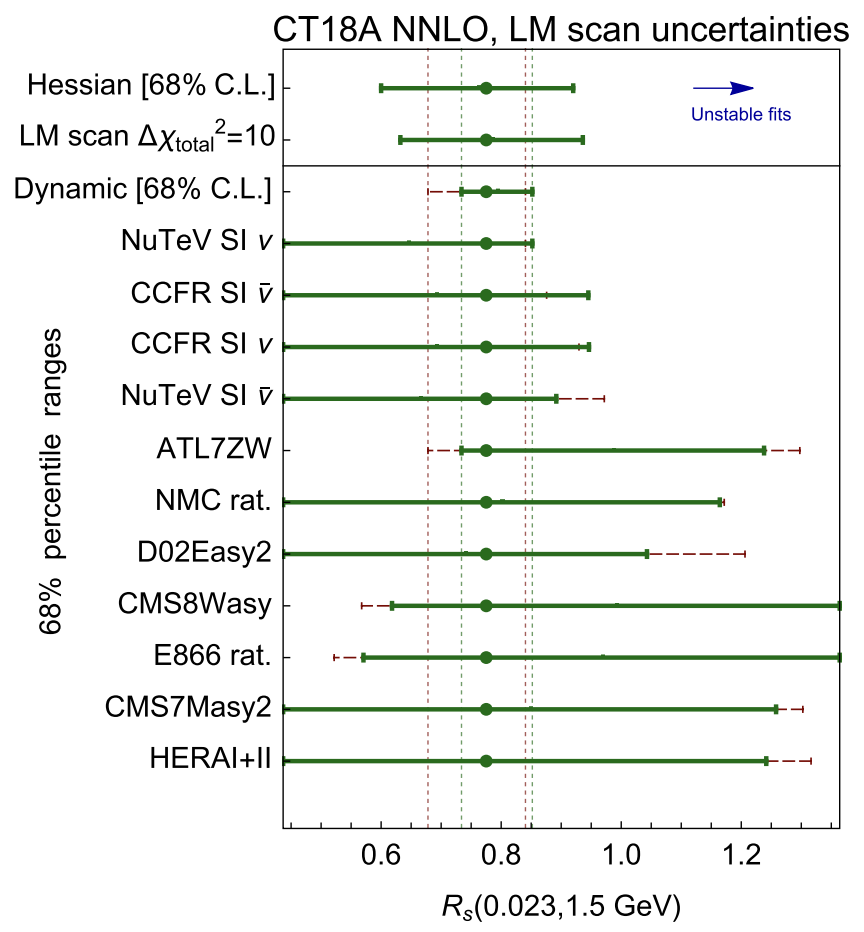

FIG. $69.68 \%$ C.L. uncertainty ranges on $R_{s}(0.023,1.5 \mathrm{GeV})$ obtained based on $\chi^{2}$ variations and Hessian estimates. For the dynamic tolerance and tolerance ranges for individual experiments, the green solid (red dashed) bands are described in the main text.
In the lower part of Fig. 69, the solid green error bands show $68 \%$ percentile ranges, $\xi_{68}$, plotted for $\chi_{E}^{2}$ distributions of individual experiments. The red dashed error bands are for these $68 \%$ percentile ranges rescaled by $\chi_{E, 0}^{2} / \xi_{50}$, where $\chi_{E, 0}^{2}$ is the $\chi^{2}$ value achieved for experiment $E$ in the best global fit. From these, we construct an estimate, in the third line, for the $68 \%$ C.L. uncertainty on $R_{S}(0.023,1.5 \mathrm{GeV})$ according to the procedure in Sec. VI 2 of the MSTW2008 analysis [166]. The resulting interval can be interpreted as the dynamic tolerance in the MSTW/ MMHT approach for a particular eigenvector direction that is chosen to be along the gradient of $R_{S}(0.023,1.5 \mathrm{GeV})$. [The other eigenvectors are perpendicular to the one shown and do not contribute to the uncertainty on $\left.R_{s}(0.023,1.5 \mathrm{GeV})\right]$.

The dynamically estimated uncertainty in the third line is substantially narrower than the two uncertainty intervals above it. The dynamic uncertainty is equal to the small overlap between the rescaled $68 \%$ percentiles for $\mathrm{NuTeV}$ neutrino and ATL7ZW datasets, which constrain the dynamic interval from above and from below. The dynamic uncertainty on $R_{S}(0.023,1.5 \mathrm{GeV})$ computed this way will tend to be too small if some experiments strongly disagree with one another. Similarly, the tier-2 penalty in the CT analysis may result in too narrow uncertainties along some eigenvector directions if some experiments strongly disagree.

\section{c. CT18 LM scans on $\alpha_{s}\left(M_{Z}\right)$ and $\boldsymbol{m}_{c}$}

The various modifications in CT18Z, especially the use of the $x_{B}$-dependent scale $\mu_{F, x}$ in DIS experiments that provide the largest coverage in the energy scale $Q$, modify the constraints on the strong coupling, $\alpha_{s}\left(M_{Z}\right)$, for which we plot the scans in Fig. 70. 

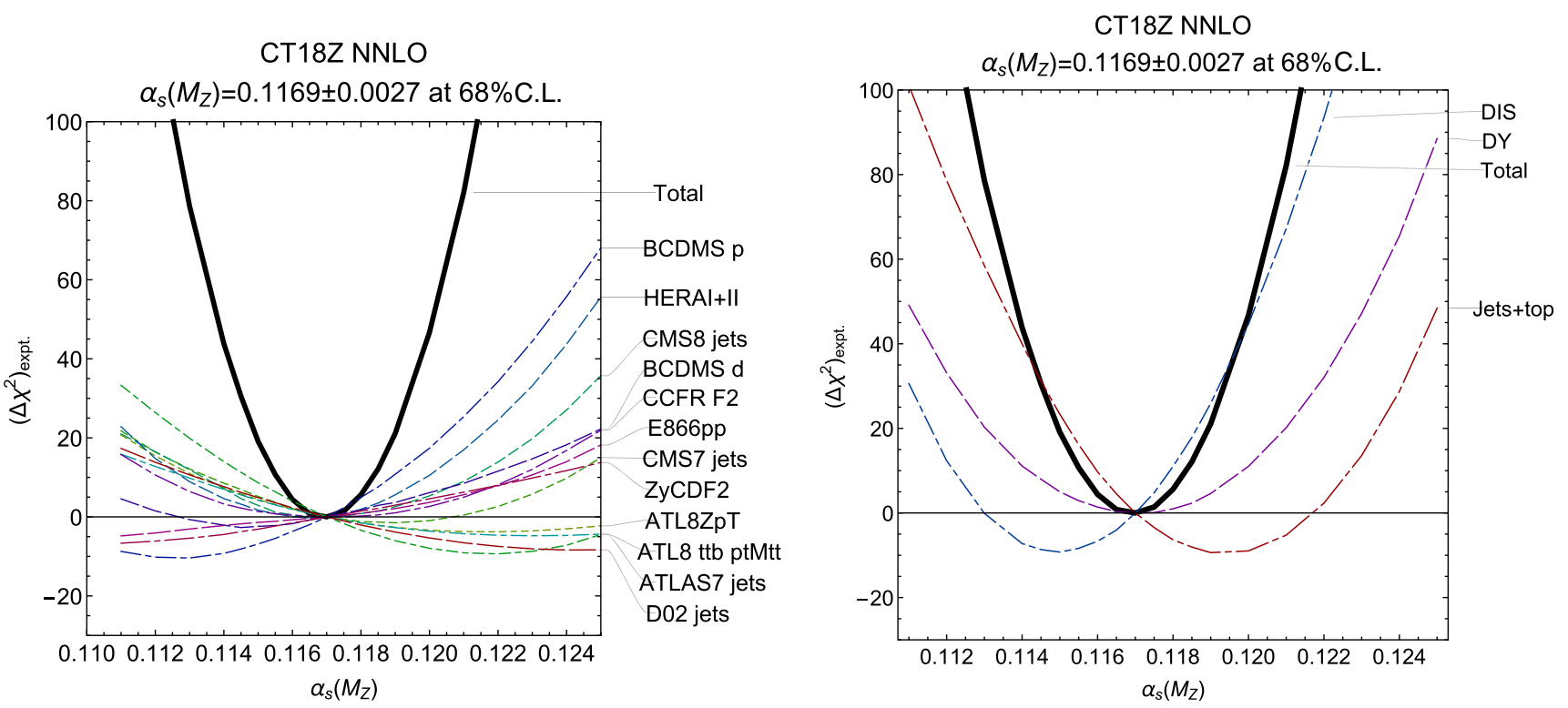

FIG. 70. Scans over the strong coupling at the scale of $M_{Z}$ for CT18Z, analogous to Fig. 13 for CT18. As before, we show in the left panel the $\Delta \chi^{2}$ variations for a number of experiments with leading sensitivity to $\alpha_{s}\left(M_{Z}\right)$, while the right panel again shows the change in $\chi^{2}$ for all experiments fitted in CT18Z, separately collected into combined DIS, DY, and top/jets datasets. Also as before, the "jets+top" curve in the right panel is primarily influenced by the jet production datasets. Due to the intermediate impact of the $t \bar{t}$ data noted in Fig. 13, variations in the CT18 fit leading to CT18Z are such that the ATLAS $8 \mathrm{TeV} t \bar{t}$ data (Exp. ID $=580)$ are now selected with the ensemble of sensitive experiments in the left panel.

Although the $68 \%$ C.L. determination of $\alpha_{s}\left(M_{Z}\right)=$ $0.1169 \pm 0.0027$ agrees closely with the CT18-based determination (with the latter being only slightly weaker and with an identical uncertainty), the experimental pulls have notable differences. Most strikingly, there is a separation in the preferences of the combined inclusive jetproduction data and Drell-Yan experiments, which were aligned closely for CT18 in Fig. 15. The combined DrellYan data (including ATL7ZW) now agree very closely with the preferred value of the full CT18Z fit, but the DIS and jet

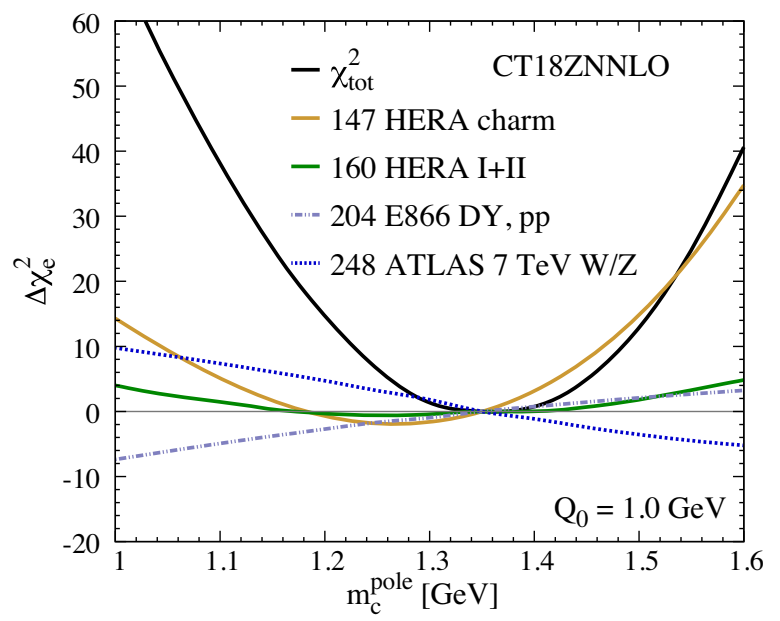

FIG. 71. Analogously to Fig. 15, the scan over values of the charm pole mass, $m_{c}$, computed here using CT18Z NNLO following the procedure described in Sec. IV B. $t \bar{t}$ data pull $\alpha_{s}\left(M_{Z}\right)$ in the opposite directions more strongly than in CT18.

Such visible dependence of the preferred ranges for $\alpha_{s}\left(M_{Z}\right)$ from three categories of experiments on the DIS QCD scale may indicate presence of important uncertainties beyond NNLO that are not accounted in the nominal $68 \%$ C.L. uncertainty of 0.0027 .

Similarly, the choices made in the alternative CT18 X/A/ $\mathrm{Z}$ global analyses can lead to different preferences in these fits for the charm pole mass, $m_{c}^{\text {pole }}$. While we described the $m_{c}$ scan in detail in the case of CT18 in Sec. IV B, repeating this scan for CT18Z NNLO leads to a somewhat different behavior shown in Fig. 71. Although CT18Z ultimately arrives at a very similar central value of $m_{c}$, the interplay of sensitive experiments now is somewhat different than that shown for CT18 in Fig. 15. The pull of the combined HERA data (160) on $m_{c}$ decreases in CT18Z, the preferred $m_{c}$ of the HERA charm-production data $($ Exp. ID $=147$ ) increases slightly. In addition, the ATL7ZW data also exhibit a modest preference for larger charm masses, and these latter two experiments produce a small increase in the central preferred value of $m_{c}$ in CT18Z relative to CT18, although with a similar extent of uncertainty.

\section{APPENDIX B: CT18 GOODNESS-OF-FIT FUNCTION AND TREATMENT OF CORRELATED UNCERTAINTIES}

In this Appendix, we summarize the implementation of the goodness-of-fit function $\chi^{2}$ and marginalization of 
nuisance parameters in the CT18 family of fits. For the latter task, we normally follow a procedure, adopted since the CTEQ6 analysis, to estimate the correlated uncertainties using the correlation matrix published by the experiment. For the few datasets that do not provide the correlation matrix, we find it convenient to present the covariance matrix in an approximate form that separates the uncorrelated and correlated components. The algorithm for this conversion is explained at the end of the Appendix.

\section{Definitions}

In Eq. (1) of Sec. III A, we introduced the standard goodness-of-fit function $\chi^{2}$ used in the recent CT fits. Here we review its treatment using a matrix notation.

We express $D_{k}, T_{k}$, and $\beta_{k \alpha}$ in units of $s_{k}$ for each $k$. That is, we introduce a vector $d \equiv S^{-1}(D-T(a))$ of length $N_{p t}$, where

$$
S \equiv \operatorname{diag}\left\{s_{1}, s_{2}, \ldots, s_{N_{p t}}\right\} .
$$

Similarly, $\lambda \equiv\left\{\lambda_{\alpha}\right\}$ is a vector of length $N_{\lambda}$; and $b \equiv S^{-1} \beta$ is a rectangular matrix of dimension $N_{p t} \times N_{\lambda}$. In this matrix notation, Eq. (1) takes the form

$$
\chi_{E}^{2}(a, \lambda)=(d-b \lambda)^{T}(d-b \lambda)+\lambda^{T} \lambda .
$$

\section{Minimization of $\chi^{2}$}

The solution for the minimal value of $\chi^{2}$ (with respect to nuisance parameters) is found in terms of the following matrices:

$$
\begin{gathered}
\mathcal{A} \equiv \mathcal{I}+b^{T} b ; \quad C \equiv I+b b^{T} ; \\
\mathcal{A}^{-1}=\mathcal{I}-b^{T} C^{-1} b ; \quad C^{-1}=I-b \mathcal{A}^{-1} b^{T} .
\end{gathered}
$$

Uppercase roman and script letters denote matrices of dimensions $N_{p t} \times N_{p t}$ and $N_{\lambda} \times N_{\lambda}$, respectively. Therefore, $I$ is an $N_{p t} \times N_{p t}$ identity matrix, and $\mathcal{I}$ is an $N_{\lambda} \times N_{\lambda}$ identity matrix. $C$ and $\mathcal{A}$ are covariance matrices (appropriately normalized by $s_{k}$ ) in spaces of data values and nuisance parameters, respectively. The relations between $\mathcal{A}^{-1}$ and $C^{-1}$ in Eq. (B4) can be demonstrated by using $b^{T} b=\mathcal{A}-\mathcal{I}$ and $b b^{T}=C-I$. The covariance matrices are symmetric: $\mathcal{A}^{T}=\mathcal{A}, C^{T}=C$.

For each theory input $a, \chi^{2}$ can be minimized with respect to $\lambda$ analytically, by solving for $\partial \chi^{2} / \partial \lambda_{\alpha}=0$ [23]. The solution is

$$
\begin{aligned}
& \bar{\lambda}(a)=\mathcal{A}^{-1} b^{T} d=b^{T} C^{-1} d ; \\
& \bar{\lambda}^{T}(a)=d^{T} b \mathcal{A}^{-1}=d^{T} C^{-1} b .
\end{aligned}
$$

The global minimum $\chi^{2}\left(a_{0}, \lambda_{0}\right)$ for all experiments $E$ can be found numerically as

$$
\chi^{2}\left(a_{0}, \lambda_{0}\right)=\sum_{E} d_{0}^{T} C^{-1} d_{0}
$$

with $d_{0} \equiv S^{-1}\left(D-T\left(a_{0}\right)\right), \lambda_{0} \equiv \bar{\lambda}\left(a_{0}\right)$.

An equivalent form of this equation can be derived,

$$
\chi^{2}\left(a_{0}, \lambda_{0}\right)=\sum_{E}\left(r_{0}^{T} r_{0}+\lambda_{0}^{T} \lambda_{0}\right)
$$

where $r_{0}$ are the best-fit shifted residuals:

$$
r_{0} \equiv S^{-1}\left(d_{0}-b \lambda_{0}\right)=\bar{r}\left(d_{0}\right), \text { with } \bar{r}(d) \equiv C^{-1} d .
$$

The representation (B8) is particularly informative. We anticipate that, in a good fit of theory to an experiment $E$, the shifted residuals $r_{0}$, quantifying agreement with individual data points, as well as the nuisance parameters $\bar{\lambda}_{0}$, quantifying the systematic shifts, are distributed according to their own standard normal distributions, $\mathcal{N}(0,1)$. Comparisons of the two empirical distributions to the expected $\mathcal{N}(0,1)$ distributions serve as the tests for the goodness of fit and for the implementation of systematic errors [37].

\section{Decomposition of the covariance matrix}

The form of $\chi^{2}$ in Eq. (8) coincides with Eq. (B7), obtained by optimizing the nuisance parameters $\lambda$ for a given $a$, in the prevalent case when we separately know the uncorrelated and fully correlated systematic errors. In this case, we identify

$$
\operatorname{cov}=S C S, \quad \operatorname{cov}^{-1}=S^{-1} C^{-1} S^{-1} .
$$

The matrix elements, according to Eqs. (B3) and (B4), are

$$
\begin{gathered}
(\mathrm{cov})_{i j}=s_{i}^{2} \delta_{i j}+\sum_{\alpha=1}^{N_{\lambda}} \beta_{i \alpha} \beta_{j \alpha}, \\
\left(\mathrm{cov}^{-1}\right)_{i j}=\frac{\delta_{i j}}{s_{i}^{2}}-\sum_{\alpha_{1}, \alpha_{2}=1}^{N_{\lambda}} \frac{\beta_{i \alpha_{1}}}{s_{i}^{2}} \mathcal{A}_{\alpha_{1} \alpha_{2}}^{-1} \frac{\beta_{j \alpha_{2}}}{s_{j}^{2}} .
\end{gathered}
$$

In particular, a diagonal element (cov) $)_{i i}$ (no summation) is the quadrature sum of the statistical, uncorrelated systematic, and correlated systematic uncertainties:

$$
(\operatorname{cov})_{i i}=s_{i, \text { stat }}^{2}+s_{i, \text { uncorsys }}^{2}+s_{i, \text { corsys }}^{2}
$$

where $s_{i, \text { corsys }}^{2} \equiv \sum_{\alpha} \beta_{i \alpha}^{2}$. With the help of Eq. (B10), the shifted residuals in Eq. (B9) then are written as

$r_{i} \equiv \frac{D_{i}^{s h}(a)-T_{i}(a)}{s_{i}}=s_{i} \sum_{j=1}^{N_{p t}}\left(\operatorname{cov}^{-1}\right)_{i j}\left(D_{i}-T_{i}(a)\right)$. 
Computing them thus requires that we know the full uncorrelated error $s_{i}=\sqrt{s_{i, \text { stat }}^{2}+s_{i, \text { uncorsys }}^{2}}$

\section{Finding a correlation matrix from the covariance matrix}

In some experimental measurements, such as the LHCb $8 \mathrm{TeV} \mathrm{W} / \mathrm{Z}$ production [84], only the full covariance matrix is provided, making impossible the straightforward reconstruction of the shifted residuals according to Eq. (B14). In several cases, we find it feasible to iteratively reconstruct the approximate uncorrelated systematic and correlated systematic contributions, $s_{k \text {,uncorsys }}$ and $\beta_{k \alpha}$, from the provided covariance matrix $\operatorname{cov} \equiv K_{0}$, by assuming that the systematic shifts are dominated by a certain number $M_{\lambda}$, with $M_{\lambda} \leq N_{\lambda}$, of fully correlated linear combinations. The approximation makes use of the positive definiteness of the covariance matrix and its diagonal elements, cf. Eq. (B13). It represents the original covariance matrix by a numerically close matrix given by the sum of a diagonal matrix $\Sigma$, interpreted as consisting of total uncorrelated errors, and a nondiagonal square matrix $K$, interpreted as a product of the correlation matrix $\beta$ and its transpose.

In particular, suppose we find the eigenvalues $x_{k}^{2}$ of $K_{0}$ and sort them in the descending order:

$$
\begin{gathered}
K_{0}=O^{T} x O, \quad \text { with } \quad x=\operatorname{diag}\left\{x_{1}^{2}, x_{2}^{2}, \ldots, x_{N_{p t}}^{2}\right\}, \\
x_{1}^{2} \geq x_{2}^{2} \geq \ldots \geq x_{N_{p t}}^{2}>0 .
\end{gathered}
$$

Here $O$ is an orthogonal matrix. We partition $x$ into a matrix $y$ containing the largest $M_{\lambda}$ eigenvalues and a matrix $z$ with the smallest $\left(N_{p t}-M_{\lambda}\right)$ ones:

$$
\begin{gathered}
y \equiv \operatorname{diag}\left\{x_{1}^{2}, x_{2}^{2}, \ldots, x_{M_{\lambda}}^{2}, 0, \ldots, 0\right\}, \\
z \equiv \operatorname{diag}\left\{0, \ldots, x_{M_{\lambda+1}}^{2}, \ldots, x_{N_{p t}}^{2}\right\} .
\end{gathered}
$$

Recalling that the diagonal elements of matrices $Y=$ $O^{T} y O$ and $Z=O^{T} z O$ are non-negative, we then construct a diagonal matrix $\Sigma_{1} \equiv \operatorname{diag}\left\{Z_{11}, \ldots, Z_{N_{p t} N_{p t}}\right\}$ with $Z_{i i}>0$, and another positive-definite matrix, $K_{1} \equiv Y+Z-\Sigma_{1}$. We can iterate the steps in Eqs. (B15)-(B18) by computing $\Sigma_{a+1}$ and $K_{a+1}$ at step $a$ as

$$
\begin{gathered}
\Sigma_{a+1} \equiv \Sigma_{a}+\operatorname{diag}\left\{Z_{11}, \ldots, Z_{N_{p t} N_{p t}}\right\}, \\
K_{a+1} \equiv Y+Z-\operatorname{diag}\left\{Z_{11}, \ldots, Z_{N_{p t} N_{p t}}\right\} .
\end{gathered}
$$

Here the matrices $Y$ and $Z$ are recomputed in each step using $K_{a}$ as the input. After a sufficient number of steps $a$, the sum

$$
C_{a} \equiv \Sigma_{a}+Y_{a}
$$

approaches an asymptotic matrix that is close to the input matrix $K_{0}$ in the sense of the $L_{p}$ norm $\sum_{i, j=1}^{N_{p t}} \mid\left(K_{0}\right)_{i j}-$ $\left.\left(C_{a}\right)_{i j}\right|^{p}$ with $p=2$ or 1 . If the extraction of the uncorrelated and fully correlated components is feasible, the asymptotic $L_{p}$ distance can be made small by choosing a large enough $M_{\lambda}$. For the three experiments $[81,83,84]$ in the CT18 dataset that provide only the covariance matrices, the $M_{\lambda}$ values giving good convergence lie in the range between $N_{\lambda} / 2$ and $N_{\lambda}$.

By comparing Eqs. (B11) and (B21), we identify

$$
\begin{gathered}
\left(\Sigma_{a}\right)_{i j} \approx s_{i}^{2} \delta_{i j}, \\
\left(Y_{a}\right)_{i j} \approx \sum_{\alpha=1}^{M_{\lambda}} \beta_{i \alpha} \beta_{j \alpha} .
\end{gathered}
$$

Hence, $s_{i}$ is estimated from $\left(\Sigma_{a}\right)_{i j}$; and $\beta_{i \alpha}$ can be estimated from $\left(Y_{a}\right)_{i j}$ by singular values decomposition.

\section{APPENDIX C: NONPERTURBATIVE PARAMETRIZATION FORMS}

As noted in Sec. III C, to obtain realistic estimates of the parametric PDF uncertainties, the CT global analyses explore a broad range of parametric forms for the PDFs at the starting scale, $Q=Q_{0}$. The goals of these investigations are (i) to select a sufficiently flexible functional form capable of fitting an expansive high-energy dataset without overfitting; and (ii) to understand the uncertainties associated with the choice of parametrization.

The Appendix in the CT14 publication [1] expounds our main rationales that guide the selection of the parametrization forms, including the ones adopted in the CT18 analysis. The general functional form in terms of the free parameters $a_{k}$ at the initial scale $Q_{0}$ is

$$
f_{i}\left(x, Q_{0}\right)=a_{0} x^{a_{1}-1}(1-x)^{a_{2}} P_{i}\left(y ; a_{3}, a_{4}, \ldots\right) .
$$

The coefficients $a_{1}$ and $a_{2}$ control the asymptotic behavior of $f_{i}\left(x, Q_{0}\right)$ in the limits $x \rightarrow 0$ and $1 . P_{i}\left(y ; a_{3}, a_{4}, \ldots\right)$ is a sum of Bernstein polynomials (also called a Bézier curve) dependent on $y=f(x)$, such as $y \equiv \sqrt{x}$, that is very flexible across the whole interval $0<x<1$. While a variety of the functional forms $P_{i}\left(x ; a_{3}, a_{4}, \ldots\right)$ has been tried at the intermediate stages, in the nominal parametrization, we use the same parameters $a_{1}$ for valence PDFs and, separately, for sea PDFs. This choice guarantees that the ratios of the respective PDFs tend to finite values in the limit $x \rightarrow 0$. We also impose similar relations on the parameters $a_{2}$ for $x \rightarrow 1$. Finally, to reduce spurious correlations between the coefficients $a_{2}$ in $(1-x)^{a_{2}}$ with the rest of the coefficients in $P_{i}\left(x ; a_{3}, \ldots\right)$ at $x \rightarrow 1$, we express some parameters in $P_{i}\left(x ; a_{3}, \ldots\right)$ in terms of the other parameters to eliminate the linear $(1-x)$ term in $P_{i}\left(x ; a_{3}, \ldots\right)$, i.e., to have 


$$
\lim _{x \rightarrow 1} x f_{i}\left(x, Q_{0}\right)=a_{0}(1-x)^{a_{2}}\left(1+\mathcal{O}\left((1-x)^{2}\right)\right) .
$$

In the CT18 case, this procedure introduces relations in Eqs. (C4) and (C6). For valence quarks, it allows us to achieve good $\chi^{2}$ values using four, rather than five or more, free parameters. The full procedure is explained around Eq. (A.16) in [1].

In CT18, the nominal nonperturbative parametrization is generally similar to the one that served as the basis for CT14 $4_{\text {HERAII }}$, but with a slightly more flexible parametrization for the sea-quark distributions. This enhanced flexibility is necessitated by the inclusion of LHC run-1 data with direct sensitivity to the sea-quark content of the nucleon. The functional form that parametrizes the startingscale valence distributions, $u_{v}$ and $d_{v}$, is a polynomial in the parameter $y \equiv \sqrt{x}$,

$$
\begin{aligned}
q_{v}\left(x, Q=Q_{0}\right)= & a_{0} x^{a_{1}-1}(1-x)^{a_{2}} P_{a}^{v}(y), \\
P_{a}^{v}(y)= & \sinh \left[a_{3}\right](1-y)^{4}+\sinh \left[a_{4}\right] 4 y(1-y)^{3} \\
& +\sinh \left[a_{5}\right] 6 y^{2}(1-y)^{2} \\
& +a_{6} 4 y^{3}(1-y)+y^{4},
\end{aligned}
$$

where

$$
a_{6}=1+\frac{1}{2} a_{1}
$$

to satisfy Eq. (C2).

We emphasize that, while the nonperturbative forms for the $u_{v}$ and $d_{v}$ distributions are the same, the parameters of $P_{a}^{v}(y)$ for these flavors are separately fitted; although, we impose constraints on the prefactor exponents, $a_{1}^{u_{v}}=a_{1}^{d_{v}}$ and $a_{2}^{u_{v}}=a_{2}^{d_{v}}$, to ensure that flavor ratios are well-behaved and consistent with Regge expectations and quark counting rules in the limits $x \rightarrow 0$ or 1 .

For the gluon PDF, we also fit a polynomial in $y=\sqrt{x}$, but with the form

$$
\begin{aligned}
g\left(x, Q=Q_{0}\right)= & a_{0} x^{a_{1}-1}(1-x)^{a_{2}} P_{a}^{g}(y), \\
P_{a}^{g}(y)= & \sinh \left[a_{3}\right](1-y)^{3}+\sinh \left[a_{4}\right] 3 y(1-y)^{2} \\
& +a_{5} 3 y^{2}(1-y)+y^{3},
\end{aligned}
$$

in which the $a_{5}$ parameter is fixed to $a_{1}$ as

\begin{tabular}{|c|c|c|c|c|c|c|}
\hline Best-fit parameters, CT18 & $u_{v}$ & $d_{v}$ & $g$ & $u_{\text {sea }}=\bar{u}$ & $d_{\text {sea }}=\bar{d}$ & $s=\bar{s}$ \\
\hline$\%$ mom. fraction & 32.5 & 13.4 & 38.5 & 2.8 & 3.6 & 1.3 \\
\hline$a_{0}$ & $3.385^{\mathrm{SR}}$ & $0.490^{\mathrm{SR}}$ & 2.690 & 0.414 & 0.414 & 0.288 \\
\hline$a_{1}$ & 0.763 & 0.763 & 0.531 & -0.022 & -0.022 & -0.022 \\
\hline$a_{2}$ & 3.036 & 3.036 & 3.148 & 7.737 & 7.737 & 10.31 \\
\hline$a_{3}$ & 1.502 & 2.615 & 3.032 & (4) & (4) & (4) \\
\hline$a_{4}$ & -0.147 & 1.828 & -1.705 & 0.618 & 0.292 & 0.466 \\
\hline$a_{5}$ & 1.671 & 2.721 & $\ldots$ & 0.195 & 0.647 & 0.466 \\
\hline$a_{6}$ & $\ldots$ & $\ldots$ & $\ldots$ & 0.871 & 0.474 & 0.225 \\
\hline$a_{7}$ & $\ldots$ & $\ldots$ & $\ldots$ & 0.267 & 0.741 & 0.225 \\
\hline$a_{8}$ & $\ldots$ & $\ldots$ & $\ldots$ & 0.733 & (1) & (1) \\
\hline CT18Z & $u_{v}$ & $d_{v}$ & $g$ & $u_{\text {sea }}=\bar{u}$ & $d_{\text {sea }}=\bar{d}$ & $s=\bar{s}$ \\
\hline$\%$ mom. fraction & 31.8 & 13.1 & 38.2 & 2.9 & 3.7 & 1.9 \\
\hline$a_{0}$ & $3.631^{\mathrm{SR}}$ & $0.254^{\mathrm{SR}}$ & 0.668 & 0.519 & 0.519 & 0.524 \\
\hline$a_{1}$ & 0.787 & 0.787 & 0.289 & 0.0096 & 0.0096 & 0.0096 \\
\hline$a_{2}$ & 3.148 & 3.148 & 1.872 & 8.27 & 8.27 & 11.4 \\
\hline$a_{3}$ & 1.559 & 3.502 & 3.538 & (4) & (4) & (4) \\
\hline$a_{4}$ & -0.075 & 1.865 & -1.665 & 0.679 & 0.300 & 0.653 \\
\hline$a_{5}$ & 1.605 & 3.599 & - & -0.0016 & 0.532 & 0.653 \\
\hline$a_{6}$ & - & - & - & 1.085 & 0.753 & 0.054 \\
\hline$a_{7}$ & - & - & - & 0.045 & 0.440 & 0.054 \\
\hline$a_{8}$ & - & - & - & 0.759 & (1) & (1) \\
\hline
\end{tabular}

TABLE X. Percent momentum fractions at $Q_{0}=1.3 \mathrm{GeV}$ and best-fit parameter values for the PDFs of the CT18 and CT18Z NNLO fits. The percent momentum fractions are evaluated as $\langle x\rangle_{f}$ based on the listed parameters for each parton flavor, such that the total seaquark percent momenta entering the momentum sum rule are twice the values given above for $u_{\text {sea }}, d_{\text {sea }}$, and $s=\bar{s}$, in each case. The functional forms associated with each parametrization are defined explicitly in the text of this section, Eqs. (C3)-(C8). Those entries corresponding to parameters that are not actively fitted for a given PDF flavor are indicated by a dash, "—-." Values in parentheses indicate fixed parameters. As indicated above with the $\#^{\mathrm{SR}}$ annotation, normalizations $a_{0}$ for $u_{v}, d_{v}$, are derived using the valence sum rules (SR), while the rest of the normalizations are derived from $\langle x\rangle_{g}$ and $\langle x\rangle_{\bar{s}+\bar{s}} /\langle x\rangle_{\bar{u}+\bar{d}}$ fitted as free parameters, and $\langle x\rangle_{\bar{u}+\bar{d}+\bar{s}}$ computed using the momentum sum rule. 


$$
a_{5}=\left(3+2 a_{1}\right) / 3
$$

For the distributions of the light-quark sea, we fit somewhat more flexible distributions relative to CT14. In these cases, we use polynomials in $y \equiv 1-(1-\sqrt{x})^{a_{3}}$ for $\bar{u}, \bar{d}$, and $\bar{s}=s$, where we fix $a_{3}=4$ for all three sea distributions. We parametrize the] sea-quark PDFs, $\bar{q}\left(x, Q_{0}\right)$ as

$$
\begin{aligned}
\bar{q}\left(x, Q=Q_{0}\right)= & a_{0} x^{a_{1}-1}(1-x)^{a_{2}} P_{a}^{\bar{q}}(y), \\
P_{a}^{\bar{q}}(y)= & (1-y)^{5}+a_{4} 5 y(1-y)^{4}+a_{5} 10 y^{2}(1-y)^{3} \\
& +a_{6} 10 y^{3}(1-y)^{2} \\
& +a_{7} 5 y^{4}(1-y)+a_{8} y^{5} .
\end{aligned}
$$

While the full parametric form of Eq. (C7) (with $a_{3}=4$ fixed) is used for the $\bar{u} \mathrm{PDF}$, for $\bar{d}$ we fix $a_{8}=1$. Owing to the comparative lack of empirical constraints to nucleon strangeness, not all the parameters above are permitted to float freely for $s(x, Q)$ in $\mathrm{CT} 18(\mathrm{Z})$; rather, we constrain $a_{4}=a_{5}, a_{6}=a_{7}$, and $a_{8}=1$ for the strangeness parameters.

As with the valence distributions, we constrain the prefactor exponents as

$$
a_{1}^{\bar{u}}=a_{1}^{\bar{d}}=a_{1}^{s},
$$

to ensure a finite value for the strangeness suppression ratio, $R_{s}=[s+\bar{s}] /[\bar{u}+\bar{d}]$, in the limit $x \rightarrow 0$, and the convergence of $\int_{0}^{1} d x[\bar{d}-\bar{u}]$. We bind the high- $x$ exponents of the $\bar{u}$ and $\bar{d}$ distributions, $a_{2}^{\bar{u}}=a_{2}^{\bar{d}}$, to stabilize $\bar{d} / \bar{u}$ for $x \rightarrow 1$. Normalizations for individual sea-quark PDFs are computed using the valence quark and momentum sum rules, and the first moments $\langle x\rangle_{g}$ and the ratio $\langle x\rangle_{\bar{s}+\bar{s}} /$ $\langle x\rangle_{\bar{u}+\bar{d}}$ fitted as free parameters. Since the parametrizations do not determine the ratio of the strange-to-nonstrange PDFs, we restrict the ratio $\left(s\left(x, Q_{0}\right)+\bar{s}\left(x, Q_{0}\right)\right) /$ $\left(\bar{u}\left(x, Q_{0}\right)+\bar{d}\left(x, Q_{0}\right)\right)$ to be in the intervals $[0.2,2.0]$ at $x=10^{-8}$, and $[0.4,1.8]$ at $x=10^{-5}$ by imposing appropriate Lagrange multiplier constraints.

In Table X, we summarize the central fitted values of the parameters noted above for CT18 (upper rows) and CT18Z (lower rows). Those parameters entries marked with "-" do not participate as degrees of freedom for the associated flavor.

\section{APPENDIX D: FITTING CODE DEVELOPMENTS}

The inclusion of more than 10 new LHC datasets into the CT18 analysis has necessitated substantial upgrades in the CTEQ global analysis software. The usage of various fast interfaces for calculations of hard-scattering cross sections became mandatory and conventional. Even after implementing the fast interfaces such as APPLGRID, the precision global fit is a time-consuming task due to the large size of experimental data and the need to explore the multiparametric probability distribution to estimate the PDF uncertainties. When transiting from CT14 to CT18, the CTEQ-TEA code was revised to parallelize various operations that were done sequentially in the past.

The computational process of a typical CTEQ-TEA global analysis can be divided into three stages, or "layers," as visualized in Fig. 72 .

The outer layer, LY0, corresponds to repeating the global fit with varied inputs or constraints, e.g., for different values

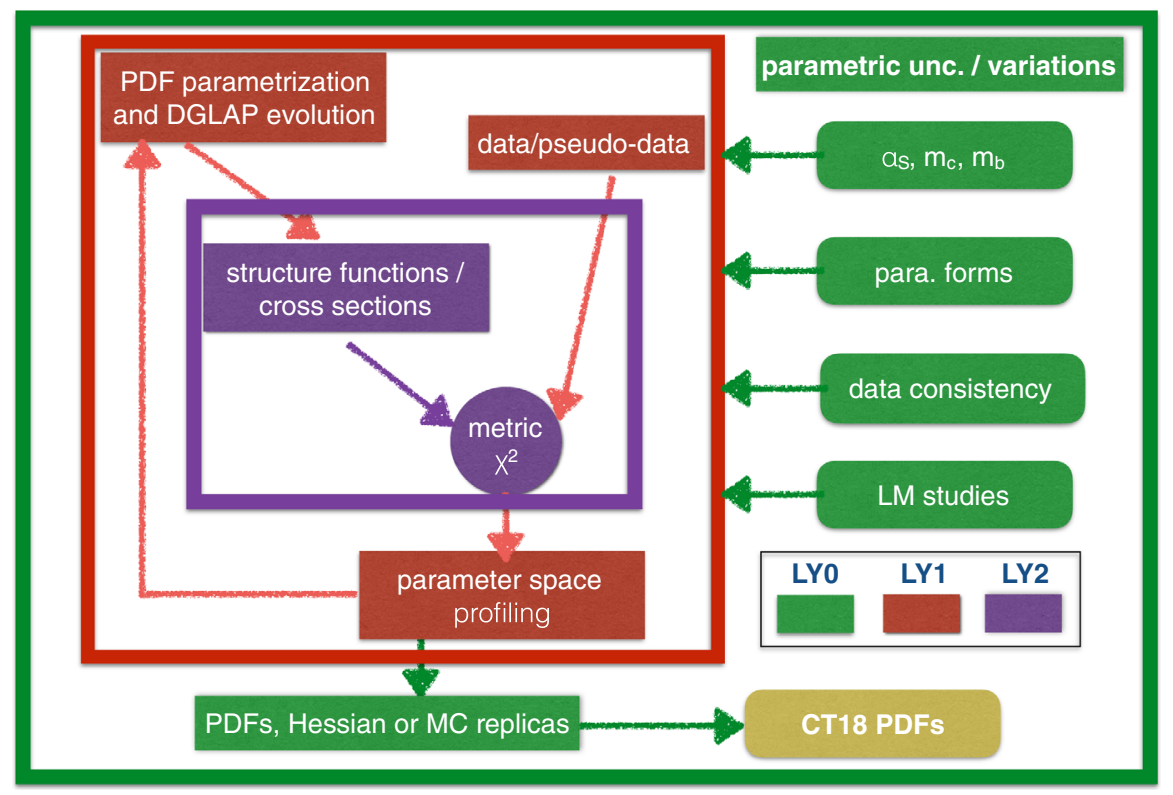

FIG. 72. A flowchart of the CT18 global analysis consists of the outer layer (LY0), middle layer (LY1), and core process (LY2). 
of the QCD coupling, heavy-quark masses, nonperturbative functional forms, or Lagrange multiplier constraints.

The middle layer, LY1, corresponds to a single such fit, in which the program scans the PDF parameter space and constructs the probability $\left(\chi^{2}\right)$ distribution for a fixed combination of inputs. This step provides the best-fit and error PDF sets that quantify the uncertainty in the parameter space.

Within the LY1 layer, the calculation of global $\chi^{2}$, or "layer LY2," is the core part of the fit that will be repeated for every combination of the PDF parameters. LY2 calculates cross sections for thousands of data points and computes individual $\chi^{2}$ for each dataset.

Most computational efforts shown in Fig. 72 can be parallelized. For instance, at the LY0 stage, one can simply submit thousands of simultaneous fits with varied inputs to a large computing cluster, since those fits are independent. To obtain a diverse battery of results presented in the CT18(Z) analyses, including the computationally expensive Lagrange multiplier scans, the high-performance computing clusters at MSU and SMU were used.

At LY1, the major task is to find a global minimum of the $\chi^{2}$ in a parameter space with large dimensions $\left(N_{p a r} \sim 30\right)$. The choice of the suitable parallelization technique depends strongly on the minimization algorithm. The CT18 analysis uses the variable-metric gradient descent method implemented in the MINUIT package [195]. It involves numerical calculations of the first- and second-order derivatives of $\chi^{2}$, combined with sequential minimum searches along fixed directions in the PDF parameter space. The calculations of derivatives are highly parallelizable, with the CPUtime expenditures scaling with the number $N_{p a r}$ of parameters approximately as $1 / N_{\text {par }}$ and $2 /\left(N_{\text {par }}^{2}+N_{\text {par }}\right)$, respectively.

In the core part, again the calculation of individual $\chi^{2}$ for different datasets, including their cross sections, is now done simultaneously. While either MPI or OPENMP parallelization protocols are suitable at this stage, the latter is restricted to platforms with shared memory, but required fewer revisions in our fitting code. Specifically, we used an approach similar to OPENMP to reduce the scope of changes inside our fitting code. When computing $\chi_{E}^{2}$ values for the experiments, the FORK Linux command splits the main program into multiple threads, each with a copy of the master memory and carrying out the calculations independently. Later the JOIN command collects the results from the individual threads and returns them to the main program. The implementation of this FORK-JOIN algorithm is borrowed from the widely used CUBA library [196] for multithread Monte Carlo integration.

\section{APPENDIX E: DECORRELATION OF ATLAS JET-PRODUCTION CROSS SECTION DATA}

It has been observed in our global analysis, as well as others [197], that achieving a robust theoretical description of the run-1 LHC jet production data generally requires numerical prescriptions to decorrelate select correlated systematic uncertainties. In our work, we follow the recommendations of experimental collaborations in applying such decorrelation procedures.

For example, while the ATLAS $7 \mathrm{TeV}$ inclusive jet production data [9] (Exp. ID = 544) produced an unacceptably high $\chi^{2}$ when fitted out of the box, the agreement of data and NNLO theory was improved by applying some decorrelation options proposed by the ATLAS collaboration and summarized in the Appendix of Ref. [10]. The CT18(Z) fits implement ATLAS inclusive jet cross sections defined with an $R=0.6$ anti- $k_{t}$ jet algorithm. With this dataset, we tested the decorrelation procedures summarized in Table 6 of Ref. [10] and found some to have a substantial impact on the $\chi_{E}^{2} / N_{p t, E}$ of the ATLAS $7 \mathrm{TeV}$ data. In the end, we followed the specific recommendations of the ATLAS experimentalists themselves, who advocate [101] the decorrelation of two JES uncertainties, associated with the JES MJB fragmentation (JES16) and the JES flavor response (JES62). We obtained the greatest $\chi^{2}$ reduction using the upper portion of Table 6 in Ref. [10], by decorrelating JES16 and JES62 according to Options 17 and 14 of Table 4, respectively.

Reference [10] also details decorrelation options for select uncertainties in experimental simulations; of these, we specifically considered the effect of decorrelating the error associated with the nonperturbative correction ("eNPC"), but found it to have negligible impact on the quality of the CT14 $14_{\text {HERAII }}$ fit according to $\chi^{2}$.

The JES decorrelation that we have implemented proceeds by breaking a given correlated uncertainty into 2-3 subsidiary errors such that their sum in quadrature recovers the original correlated uncertainty. This procedure is bindependent; the resulting decorrelated errors depend on the jet's rapidity and transverse momentum. For example, we decorrelate the JES MJB fragmentation error, $\delta_{16}$, into three components given by

$$
\begin{aligned}
\delta_{16}^{a}= & \delta_{16}\left\{1-L^{2}\left(\ln \left(p_{T}[\mathrm{TeV}]\right), \ln (0.1),\right.\right. \\
& \ln (2.5))\}^{1 / 2}\left(1-L^{2}(|y|, 0,1)\right)^{1 / 2}, \\
\delta_{16}^{b}= & \delta_{16}\left\{1-L^{2}\left(\ln \left(p_{T}[\mathrm{TeV}]\right), \ln (0.1),\right.\right. \\
& \ln (2.5))\}^{1 / 2} L(|y|, 1,3), \\
\delta_{16}^{c}= & \left(\delta_{16}^{2}-\left(\delta_{16}^{a}\right)^{2}-\left(\delta_{16}^{b}\right)^{2}\right)^{1 / 2},
\end{aligned}
$$

where

$L\left(x, x_{\min }, x_{\max }\right) \equiv \frac{x-x_{\min }}{x_{\max }-x_{\min }} \Leftrightarrow x \in\left[x_{\min }, x_{\max }\right]$,

and otherwise $L=0$ for $x<x_{\min }$ or $L=1$ for $x>x_{\max }$. We note that Eq. (E1) governs the magnitudes of the decorrelated uncertainties, which otherwise inherit the sign 
TABLE XI. Values of $\chi_{E}^{2} / N_{p t, E}$ for the inclusive jet production data implemented in CT18, computed here using the CT14 $4_{\text {HERAII }}$ PDFs [32] under several error treatment scenarios. $\chi_{E}^{2} / N_{p t, E}$ is first given without implementing any decorrelation scheme ("original data"); using the decorrelation scheme described above ("+ decorr."); and, finally, adding an overall uncorrelated Monte Carlo uncertainty of $0.5 \%$ on the top of decorrelations (“+0.5\% MC unc.").

\begin{tabular}{lccc}
\hline \hline & \multicolumn{3}{c}{ Evaluated, CT14 HERAII NNLO } \\
\cline { 2 - 4 }$\chi_{E}^{2} / N_{p t, E}$ & Original data & + decorr. & $+0.5 \%$ MC unc. \\
\hline ATLAS, $7 \mathrm{TeV}$ & 2.34 & 1.68 & 1.31 \\
CMS, $7 \mathrm{TeV}$ & 1.58 & 1.45 & 1.35 \\
$\mathrm{CMS}, 8 \mathrm{TeV}$ & 1.90 & 1.34 & 1.23 \\
\hline \hline
\end{tabular}

of the original uncertainty. A similar algorithm is applied to decorrelate the JES flavor response JES62.

Analogous considerations were applied to the inclusive jet production datasets from CMS 7 and $8 \mathrm{TeV}$ (experiments 542 and 545). Namely, for the $7 \mathrm{TeV}$ CMS jet data, the decorrelation methods of Ref. [97] were applied to JEC2 with an additional decorrelation ${ }^{8}$ for the $2.5<|y|<$ 3.0 bin to obtain six subsidiary uncertainties. For the $8 \mathrm{TeV}$ CMS jet data, systematic uncertainties were obtained using the xFitter framework according to the treatment in Ref. [198]. On the top of that, we found that the residual fluctuations from the Monte Carlo integration of the available NNLO predictions from NNLOJET $[11,12,52,53]$ lead to elevated $\chi^{2}$ for the inclusive jet production experiments, or equivalently, to some uncertainty in the tabulated NNLO/NLO corrections if they are fitted by a smooth function. In the CT18 analysis, the MC theoretical uncertainty for the three jet experiments is estimated by adding an overall uncorrelated uncertainty ("MC unc.") of $0.5 \%$, the typical magnitude of the intrinsic statistical noise associated with Monte-Carlo generation of NNLO/NLO $K$ factors.

Table XI summarizes the reduction in $\chi_{E}^{2} / N_{p t, E}$ for the three LHC jet datasets after performing the decorrelation and adding the Monte Carlo uncertainties, on the example of the CT14 ${ }_{\text {HERAII }}$ PDFs. For example, for the ATLAS $7 \mathrm{TeV}$ jet data, the decorrelation reduces $\chi_{E}^{2}$ by $\gtrsim 90$ units, yet adding the MC uncertainty is still necessary to reduce the $\chi_{E}^{2} / N_{p t, E}$ to a statistically plausible level (from 1.68 to 1.31 for $N_{p t, E}=140$ ).

\section{APPENDIX F: HESSIAN PROFILING OF THE ATLAS 7 TEV $W / Z$ DATA}

\section{The $\chi^{2}$ definitions in xFitter and EPUMP}

As an alternative to directly including new data inside a full QCD global fit, Hessian PDF-profiling techniques

\footnotetext{
${ }^{8}$ Private communication, M. Voutilainen.
}

provide a fast and flexible approach to explore the impact of new data on a given PDF set; these profiling methods are available within both the xFitter and EPUMP frameworks. In xFitter, the $\chi^{2}$ function includes both experimental and theoretical uncertainties [199-201]:

$$
\begin{aligned}
\chi^{2}\left(\vec{\lambda}_{\exp }, \vec{\lambda}_{\mathrm{th}}\right)= & \sum_{i=1}^{N_{p t}} \frac{\left[D_{i}+\sum_{\alpha} \beta_{i, \alpha}^{\exp } \lambda_{\alpha, \exp }-T_{i}-\sum_{\alpha} \beta_{i, \alpha}^{\text {th }} \lambda_{\alpha, \mathrm{th}}\right]^{2}}{s_{i}^{2}} \\
& +\sum_{\alpha} \lambda_{\alpha, \exp }^{2}+\sum_{\alpha} T^{2} \lambda_{\alpha, \mathrm{th}}^{2} .
\end{aligned}
$$

Here $D_{i}$ and $T_{i}$ are the $i$ th experimental datum and corresponding theoretical prediction, respectively, where the index $i$ runs over all $N_{p t}$ points in a given experiment. Meanwhile, $s_{i}$ denotes the total uncorrelated uncertainty, $\beta_{i, \alpha}^{\exp }\left(\beta_{i, \alpha}^{\text {th }}\right)$ represents the correlated experimental (theoretical) uncertainties, and $\lambda_{\alpha, \exp }\left(\lambda_{\alpha, \mathrm{th}}\right)$ are the corresponding nuisance parameters. In this case, the index $\alpha$ runs over the $N_{\lambda}$ correlated systematic uncertainties in an experiment, or over the $N_{e v}$ Hessian eigenvector directions of the PDF error sets. Theoretical uncertainties are determined according to predictions based upon the corresponding PDF error sets, $f_{\alpha}^{ \pm}$, as

$$
\beta_{i, \alpha}^{\mathrm{th}}=\frac{T_{i}\left(f_{\alpha}^{+}\right)-T_{i}\left(f_{\alpha}^{-}\right)}{2},
$$

in which $f_{\alpha}^{ \pm}$are the PDF error sets corresponding to positive and negative variations along eigenvector $\alpha$. The tolerance parameter $T$ is set to 1 or 1.645 if the PDF error sets are defined at the $68 \%$ or $90 \%$ C.L., respectively. Instead of scaling down the theoretical uncertainties $\beta_{i, \alpha}^{\text {th }}$ as in Ref. [201], we equivalently scale up the corresponding nuisance parameters $\lambda_{\alpha, \text { th }}$ in order to compare with EPUMP $[24,25]$ more transparently.

The $\chi^{2}$ function in Eq. $(\mathrm{F} 1)$ can be converted into the form defined in EPUMP $[24,25]$ :

$$
\begin{aligned}
\Delta \chi^{2}\left(\vec{\lambda}_{\mathrm{th}}\right)= & \sum_{i, j=1}^{N_{p t}}\left[D_{i}-T_{i}\left(\vec{\lambda}_{\mathrm{th}}\right)\right] \operatorname{cov}_{i j}^{-1}\left[D_{j}-T_{j}\left(\vec{\lambda}_{\mathrm{th}}\right)\right] \\
& +\sum_{\alpha} T^{2} \lambda_{\alpha, \mathrm{th}}^{2}
\end{aligned}
$$

where $\operatorname{cov}_{i j}^{-1}$ is the inverse experimental covariance matrix constructed from $s_{i}$ and $\beta_{i, \alpha}^{\exp }$ [42]. More generally, EPUMP also contains the option of the so-called dynamical tolerance $T^{(\alpha)}$ [24,25], whose specific value depends on the corresponding PDF eigenvector direction $\alpha$, for the purpose of taking into account additional constraints on the PDF error sets. For example, in the CTEQ-TEA approach, the displacement along a given eigenvector direction is constrained either by the increase in the global $\chi^{2}$ by 100 units (at $90 \%$ probability level) or by the tier- 2 penalty for a too 
TABLE XII. The $\chi^{2}$ values for the ATLAS $7 \mathrm{TeV} W / Z$ data before and after xFitter profiling and EPUMP updating, using the CT14 and CT18 PDFs.

\begin{tabular}{|c|c|c|c|c|c|}
\hline \multirow{2}{*}{$\frac{\text { Program }}{\text { PDFs }}$} & \multicolumn{2}{|c|}{ xFitter } & \multicolumn{3}{|c|}{ EPUMP } \\
\hline & Input & Profiled & Input & $\begin{array}{l}\text { Updated with } T_{2}=1.645^{2}, \\
\text { as in xFitter }\end{array}$ & $\begin{array}{l}\text { Updated with dynamical } \\
\text { tolerance, as in CT18 fit }\end{array}$ \\
\hline \multicolumn{6}{|c|}{ All the seven measurements, $N_{\mathrm{pt}}=61$} \\
\hline CT14 & 290 & 106 & 285 & 104 & 197 \\
\hline CT18 & 362 & 104 & 356 & 103 & 199 \\
\hline \multicolumn{6}{|c|}{$W^{+}, W^{-}, Z$-peak DY (central), $N_{\mathrm{pt}}=34$} \\
\hline CT14 & 224 & 66 & 220 & 66 & 140 \\
\hline CT18 & 294 & 63 & 289 & 63 & 144 \\
\hline
\end{tabular}

large increase in $\chi_{E}^{2}$ for one of the experiments [38,42], which results in a $T^{(\alpha)}<100$. Dynamical tolerance is applied to MMHT PDFs as well, where separate constraints come from individual experiments [2].

The minimum of the $\chi^{2}$ function quantifies the compatibility between theory and data, and the minimization of $\chi^{2}$ optimizes the PDFs to describe the data $[24,199]$. In the linear approximation, the "updated" (or, in the language of xFitter, the "profiled") central PDF set, $f_{0}^{\prime}$, can be given in terms of nuisance parameters, $\lambda_{\alpha, \text { th }}^{\min }$, at the $\chi^{2}$ minimum:

$$
f_{0}^{\prime}=f_{0}+\sum_{\alpha} \lambda_{\alpha, \mathrm{th}}^{\min } \frac{f_{\alpha}^{+}-f_{\alpha}^{-}}{2} .
$$

The xFitter-profiled PDFs also include second-order diagonal terms, $\sim \frac{1}{2}\left(\lambda_{\alpha, \text { th }}^{\min }\right)^{2}\left(f_{\alpha}^{+}+f_{\alpha}^{-}-2 f_{0}\right)$. We note that the offdiagonal terms, $\sim \lambda_{\alpha, \text { th }}^{\min } \lambda_{\alpha^{\prime} \text {,th }}^{\min }$ for $\alpha \neq \alpha^{\prime}$, are not presently included, since the off-diagonal, second-order partial derivatives cannot be constructed solely in terms of Hessian PDF error sets [151].

\section{Impact of the ATLAS $W / Z$ data}

Here, we use the Hessian-profiling method of xFitter, as well as EPUMP updating, to explore the impact of the ATLAS $7 \mathrm{TeV}$ inclusive $W / Z$-production data (Exp. $\mathrm{ID}=248$, [39]) on several PDF sets. The change in the total $\chi^{2}$ values before and after profiling/updating with the ATLAS $7 \mathrm{TeV} Z / W$-production data is presented in Table XII for each PDF set. First, we explore all seven measurements, having a total of $N_{p t}=61$ data points: $W^{+}$, $W^{-}$, neutral current DY in the low-mass, Z-peak, and highmass regions for the central and forward selections. In this case, we can directly compare with the ATLAS [39] and MMHT [19] analyses. As a second case, we take only the three most precise measurements, i.e., the $W^{+}, W^{-}$, and $Z$-peak DY data for the central selection, with $N_{p t}=34$ data points in total, which are included in the NNPDF3.1 [3] and $\mathrm{CT} 18 \mathrm{~A}(\mathrm{Z})$ global analyses. The comparison of the fitted $\chi^{2} / N_{p t}$ values for these data in CT18A(Z), NNPDF3.1 and MMHT can be found in Sec. A 3 .

Table XII shows that the $\chi^{2}$ values of CT14, before and after xFitter profiling, agree well with the results presented in Ref. [39]. Here, we should apply the tolerance $T=$ 1.645 in xFitter as the CT PDFs are defined according to a $90 \%$ C.L. [1,32]. As shown in Ref. [25], the same results can be reproduced by the EPUMP code when the tolerance is set to $T=1.645$. However, as clearly discussed in [25], setting $T=1.645$ in the EPUMP calculation for the CT $\mathrm{PDF}$ is equivalent to assigning a very large weight (about $100 / 1.645^{2}$ ) to the new dataset included in the fit. xFitter profiling therefore generally overestimates the impact of new datasets when using the CT PDFs. Meanwhile, a universal tolerance value $(T=1.645)$ is not able to capture the constraints of tier- 2 penalty to determine the CT PDF error sets. An appropriate way to update an existing CT PDF set with the inclusion of any new experimental data is to adopt a dynamical tolerance. As such, xFitter profiling yields a smaller $\chi^{2}$ value (63) than does EPUMP updating with a dynamical tolerance (144), as can be seen by comparing the rightmost entries in the last row of Table XII. This conclusion also holds when using xFitter profiling with the MMHT [2] and PDF4LHC15 [152] PDFs.

The $\chi^{2}$ value of the 34 highest-precision ATLAS $7 \mathrm{TeV} W / Z$-production data points is found to be $\chi^{2}=$ 87.6 in the CT18A global fit, cf. Table IX. To this we compare the corresponding value found using EPUMP updating with dynamical tolerance, for which we obtain $\chi^{2}=144$, as reported in Table XII. This value includes two distinct contributions, $\chi_{1}^{2}=104$ from the difference between theory and data of the ATLAS $7 \mathrm{TeV} W / Z$-production itself, as well as from the quadrature sum $\chi_{2}^{2}=\sum_{\alpha} \lambda_{\alpha, \text { th }}^{2}=40$ of theoretical nuisance parameters, which can be interpreted as the increase in $\chi^{2}$ of the other ("prior") datasets included in the CT18 fit. The large increase in $\chi_{2}^{2}$, after EPUMP updating, indicates the presence of some tensions 

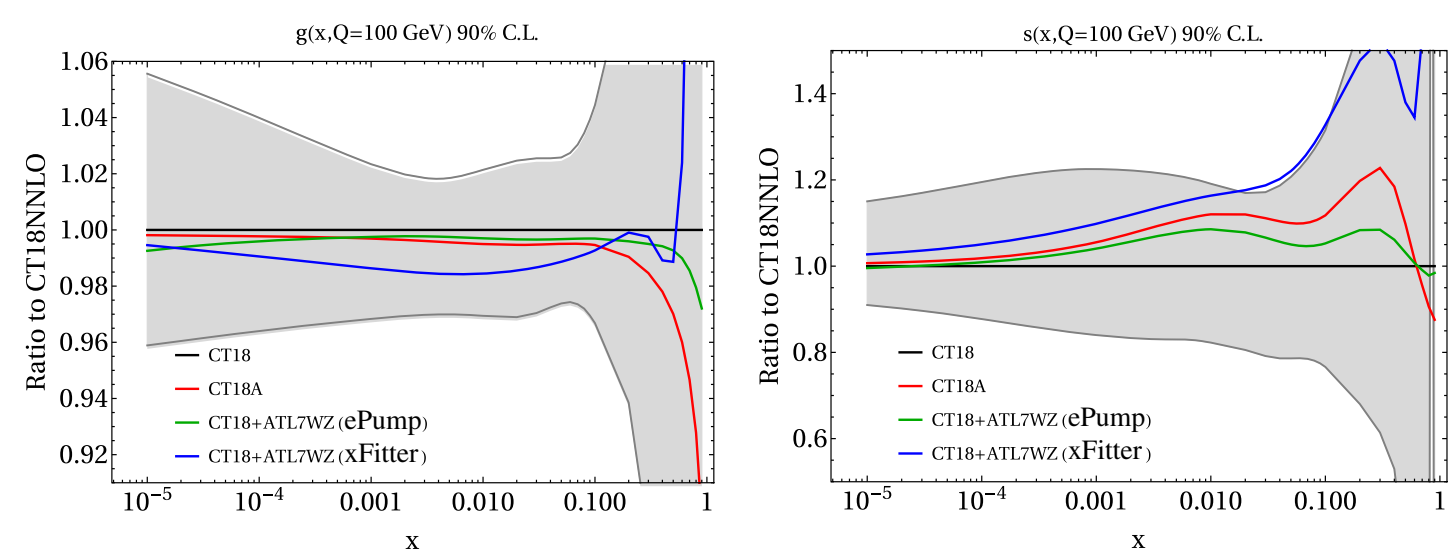

FIG. 73. Gluon and strangeness PDFs at $Q=100 \mathrm{GeV}$ for the CT18 (at 90\% C.L.) and CT18A global fits, compared with the respective central PDFs obtained by EPUMP dynamical-tolerance updating and the xFitter profiling of the CT18 PDFs by the ATLAS $7 \mathrm{TeV} W / Z$-production data. The xFitter-profiled PDFs are obtained with $T=1.645$ and include the diagonal second-order terms.

between the ATLAS $7 \mathrm{TeV} W / Z$-production data and the prior datasets, such as the CCFR/NuTeV SIDIS dimuon data [25], cf. Fig. 67.

The differences between the $\chi^{2}$ values in CT18A (87.6) and from EPUMP updating with a dynamical tolerance (104) indicate the breakdown of the linear approximation used in the Hessian-updating method, when applied to this case. The breakdown can be confirmed by examining the updated central PDF set. In Fig. 73, we show the gluon and strangeness PDFs at $Q=100 \mathrm{GeV}$ for the CT18 (with 90\% C.L. error) and CT18A global fits, compared with EPUMP dynamicaltolerance updating and xFitter profiling of CT18 with the ATLAS $7 \mathrm{TeV} W / Z$-production data. As compared to the CT18 PDFs, we see that the updated $g$ and $s$ PDFs from the EPUMP program are similar to the CT18A PDFs, although with somewhat smaller shifts in the data-sensitive range, $10^{-3}<x<10^{-1}$. In contrast, the default xFitter profiling produces a much larger shift in both $g$ and $s$ PDFs, than the CT18A global fit. As a result, xFitter profiling produces a too large change in the $s$ PDF, so that its central prediction touches the upper error band of CT18 at $x>0.02$. We have found similar features in the comparison of other flavor PDFs. Namely, the default xFitter program generally overestimates the impact of new datasets in updating the existing PDFs [25].

\section{Comparison of different NNLO predictions}

In addition to the studies described above, we have also used the xFitter and EPUMP frameworks to examine aspects of the theory calculations for the ATLAS $7 \mathrm{TeV} W / Z$ data. In the CT18A(Z) global fits, the NNLO predictions for these measurements were calculated using NNLO/NLO $K$ factors combined with NLO APPLGRID predictions. Specifically, the $K$ factors used in our CT18A(Z) fits were directly extracted from xFitter, where they were calculated with the DYNNLO code $[44,45]$. It was noted by the ATLAS Collaboration in Ref. [39] that the integrated fiducial $Z$,
$W^{+}$, and $W^{-}$cross sections predicted by the NNLO codes FEWZ [49-51] and DYNNLO $[44,45]$ differ by about $0.2 \%$, $1.2 \%$, and $0.7 \%$, respectively. Figure 74 shows a slightly larger difference, since the DYNNLO curves include the NLO EW corrections, while other two do not.

Next, we wish to compare various NNLO predictions for differential cross section measurements of the ATLAS $7 \mathrm{TeV} W / Z$-production data. First, we verified that the NLO predictions agree, within $0.2 \%$, among DYNNLO, MCFM [47,48], and FEWZ, which reflects the fact that all three codes adopt the dipole formalism [202,203] in the NLO calculations. Since the NNLO predictions can be expressed as the NLO predictions multiplied by their $K$ factors, we compare in Fig. 74 the $K$ factors obtained from each code, for every ATLAS $7 \mathrm{TeV} W / Z$-production data point (with 34 data points in total). It can be seen that the differences among the three $K$ factors vary as a function of the $Z$-boson rapidity, or the rapidity of the charged lepton from the $W$-boson decay. These differences can be sizable, $\gtrsim 1 \%$, as compared to the typically subpercent statistical uncertainty found in the ATLAS $7 \mathrm{TeV} W / Z$ data. We also find that the predictions of MCFM generally lie between those of DYNNLO and FEWZ. This difference can be understood as a consequence of the different NNLO techniques: FEWZ adopts sector decomposition [204-206], while DYNNLO and MCFM are based on transverse-momentum $\left(q_{T}\right)$ [44,45] and $N$-jettiness $\left(\mathcal{T}_{N}\right)$ [47] subtractions, respectively.

It is also useful to investigate the dependence of the ATLAS $7 \mathrm{TeV} W / Z$ fit quality on the specific choice of NNLO calculation scheme. Table XIII summarizes the findings of such a study. After CT18 PDFs are updated using EPUMP with the ATLAS $7 \mathrm{TeV} W / Z$ data, we find that the final $\chi^{2}$ value for the ATLAS $W / Z$ dataset is equal to 144,144 , and 135 units when theory is predicted by the DYNNLO, MCFM, and FEWZ NNLO calculations, respectively. Hence, we conclude that these three NNLO calculations lead to fits of similar quality for the ATLAS $7 \mathrm{TeV}$ 

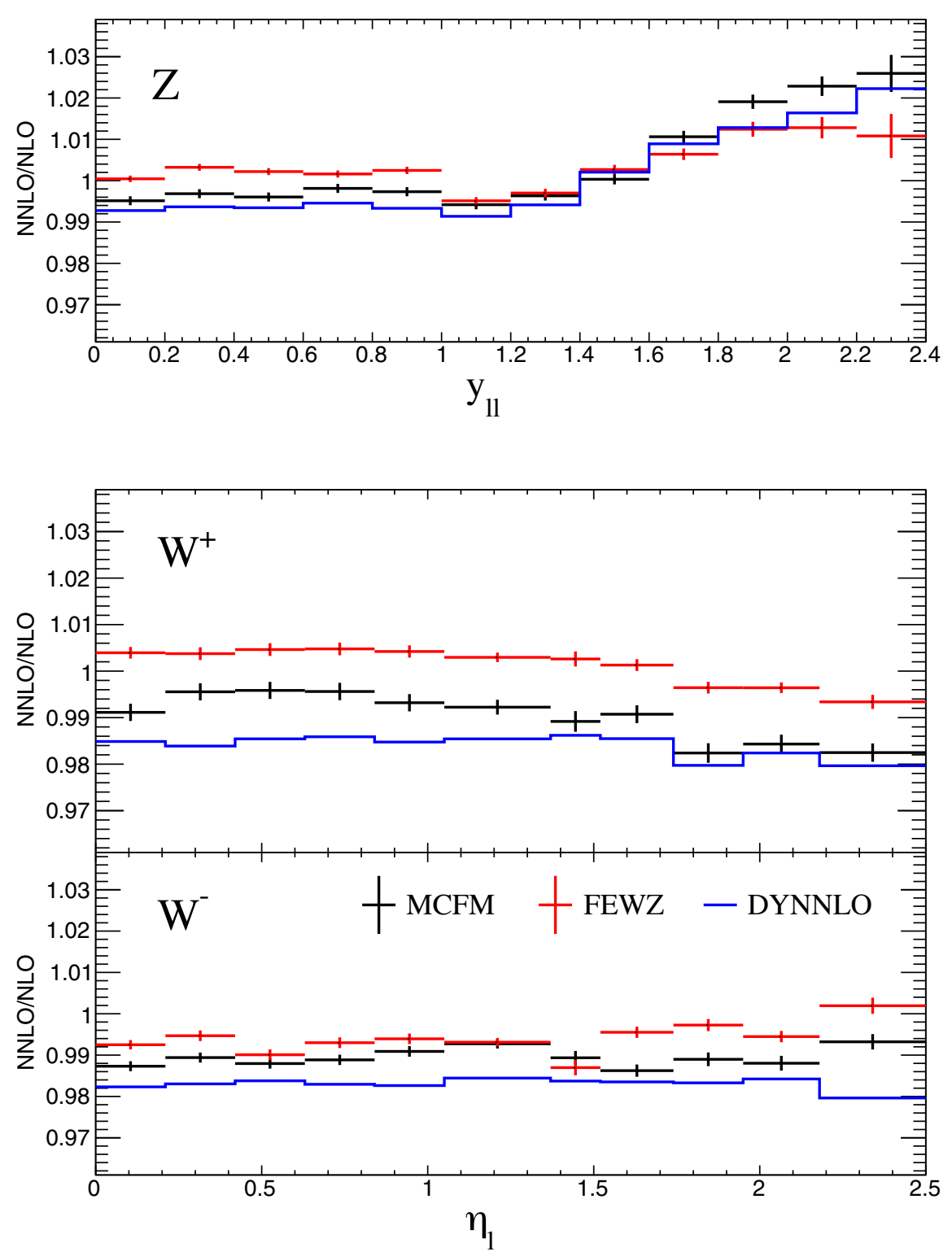

FIG. 74. The comparison of $K$ factors for the ATLAS $7 \mathrm{TeV} W / Z$ data calculated with DYNNLO, FEWZ, and MCFM. The error bars indicate the theoretical Monte Carlo uncertainties. The DYNNLO curves are extracted from xFitter and include NLO EW corrections.

TABLE XIII. The $\chi^{2}$ values for the ATLAS $7 \mathrm{TeV} W / Z$-production data (with 34 data points in total), before and after the xFitter profiling and the EPUMP dynamical-tolerance updating, with various NNLO predictions. (See the text for details.) We do not update $\mathrm{CT18A}(\mathrm{Z})$ fits to avoid double counting the impact of the ATLAS $7 \mathrm{TeV} W / Z$-production data.

\begin{tabular}{|c|c|c|c|c|c|c|}
\hline \multirow[b]{2}{*}{ xFitter profiling } & \multicolumn{2}{|c|}{ DYNNLO } & \multicolumn{2}{|c|}{ MCFM } & \multicolumn{2}{|c|}{ FEWZ } \\
\hline & & & & & & \\
\hline PDF & Before & After & Before & After & Before & After \\
\hline CT18 & 294 & 63 & 277 & 65 & 225 & 62 \\
\hline CT18A & 87 & $\ldots$ & 92 & $\ldots$ & 109 & $\ldots$ \\
\hline CT18Z & 88 & $\ldots$ & 94 & $\ldots$ & 109 & $\ldots$ \\
\hline \multicolumn{7}{|c|}{ EPUMP dynamic-tolerance updating } \\
\hline CT18 & 289 & 144 & 273 & 144 & 223 & 135 \\
\hline CT18A & 87 & $\ldots$ & 91 & $\ldots$ & 109 & $\ldots$ \\
\hline CT18Z & 88 & $\ldots$ & 94 & $\ldots$ & 109 & $\ldots$ \\
\hline
\end{tabular}


$W / Z$ data. A similar conclusion also holds when using the xFitter framework, with the final $\chi^{2}$ values being 63,65 , and 62 units, respectively. To recap, the default xFitter profiling (with tolerance $T=1.645$ ) overestimates the impact of the ATLAS $7 \mathrm{TeV} W / Z$ data when updating the CT18 PDFs, explaining why it yields a smaller $\chi^{2}$ value than EPUMP updating. We also confirm that the updated PDFs by the three $K$ factors differ slightly, but the difference is negligible compared with the same systematical shifts due to the experimental uncertainties. In conclusion, the NNLO theoretical predictions for the ATLAS $7 \mathrm{TeV} W / Z$ data by DYNNLO, MCFM, and FEWZ show perceptible differences when using the same PDF set. On the other hand, after the PDFs are updated by either EPUMP and xFitter, the final $\chi^{2}$ and PDFs show minor differences among the three codes.
[1] S. Dulat, T.-J. Hou, J. Gao, M. Guzzi, J. Huston, P. Nadolsky, J. Pumplin, C. Schmidt, D. Stump, and C.-P. Yuan, Phys. Rev. D 93, 033006 (2016).

[2] L. A. Harland-Lang, A. D. Martin, P. Motylinski, and R. S. Thorne, Eur. Phys. J. C 75, 204 (2015).

[3] R. D. Ball et al. (NNPDF Collaboration), Eur. Phys. J. C 77, 663 (2017).

[4] S. Alekhin, J. Blümlein, S. Moch, and R. Placakyte, Phys. Rev. D 96, 014011 (2017).

[5] A. Accardi, L. T. Brady, W. Melnitchouk, J. F. Owens, and N. Sato, Phys. Rev. D 93, 114017 (2016).

[6] L. A. Harland-Lang, A. D. Martin, R. Nathvani, and R. S. Thorne, Eur. Phys. J. C 79, 811 (2019).

[7] V. Bertone, S. Carrazza, N. P. Hartland, and J. Rojo (NNPDF Collaboration), SciPost Phys. 5, 008 (2018).

[8] A. V. Manohar, P. Nason, G. P. Salam, and G. Zanderighi, J. High Energy Phys. 12 (2017) 046.

[9] G. Aad et al. (ATLAS Collaboration), J. High Energy Phys. 02 (2015) 153; 09 (2015) 141(E).

[10] M. Aaboud et al. (ATLAS Collaboration), J. High Energy Phys. 09 (2017) 020.

[11] J. Currie, E. W. N. Glover, and J. Pires, Phys. Rev. Lett. 118, 072002 (2017).

[12] J. Currie, E. W. N. Glover, T. Gehrmann, A. Gehrmann-De Ridder, A. Huss, and J. Pires, Acta Phys. Pol. B 48, 955 (2017).

[13] J. Currie, A. Gehrmann-De Ridder, T. Gehrmann, E. W. N. Glover, A. Huss, and J. Pires, J. High Energy Phys. 10 (2018) 155.

[14] D. Britzger, K. Rabbertz, F. Stober, and M. Wobisch (fastNLO Collaboration), arXiv:1208.3641.

[15] M. Wobisch, D. Britzger, T. Kluge, K. Rabbertz, and F. Stober (fastNLO Collaboration), arXiv:1109.1310.

[16] T. Carli, D. Clements, A. Cooper-Sarkar, C. Gwenlan, G. P. Salam, F. Siegert, P. Starovoitov, and M. Sutton, Eur. Phys. J. C 66, 503 (2010).

[17] M. Czakon, D. Heymes, and A. Mitov, arXiv:1704.08551.

[18] http://www.precision.hep.phy.cam.ac.uk/results/ttbarfastnlo/.

[19] R. S. Thorne, S. Bailey, T. Cridge, L. A. Harland-Lang, A. D. Martin, and R. Nathvani, arXiv:1907.08147.

[20] B.-T. Wang, T. J. Hobbs, S. Doyle, J. Gao, T.-J. Hou, P. M. Nadolsky, and F. I. Olness, Phys. Rev. D 98, 094030 (2018).
[21] T. J. Hobbs, B.-T. Wang, P. M. Nadolsky, and F. I. Olness, arXiv:1904.00022.

[22] J. Pumplin, D. Stump, R. Brock, D. Casey, J. Huston, J. Kalk, H.-L. Lai, and W.-K. Tung, Phys. Rev. D 65, 014013 (2001).

[23] J. Pumplin, D. R. Stump, J. Huston, H. L. Lai, P. M. Nadolsky, and W.-K. Tung, J. High Energy Phys. 07 (2002) 012.

[24] C. Schmidt, J. Pumplin, and C.-P. Yuan, Phys. Rev. D 98, 094005 (2018).

[25] T.-J. Hou, Z. Yu, S. Dulat, C. Schmidt, and C. P. Yuan, arXiv:1907.12177.

[26] D. Stump, J. Pumplin, R. Brock, D. Casey, J. Huston, J. Kalk, H.-L. Lai, and W.-K. Tung, Phys. Rev. D 65, 014012 (2001).

[27] See Supplemental Material at http://link.aps.org/ supplemental/10.1103/PhysRevD.103.014013 for additional figures that were not included in the main paper.

[28] M. Tanabashi et al. (Particle Data Group), Phys. Rev. D 98, 030001 (2018).

[29] H.-L. Lai, J. Huston, Z. Li, P. Nadolsky, J. Pumplin, D. Stump, and C. P. Yuan, Phys. Rev. D 82, 054021 (2010).

[30] H. Abramowicz et al. (H1, ZEUS Collaborations), Eur. Phys. J. C 75, 580 (2015).

[31] F. D. Aaron et al. (H1, ZEUS Collaborations), J. High Energy Phys. 01 (2010) 109.

[32] T.-J. Hou, S. Dulat, J. Gao, M. Guzzi, J. Huston, P. Nadolsky, J. Pumplin, C. Schmidt, D. Stump, and C. P. Yuan, Phys. Rev. D 95, 034003 (2017).

[33] F. D. Aaron et al. (H1 Collaboration), Eur. Phys. J. C 71, 1579 (2011).

[34] R. D. Ball, V. Bertone, M. Bonvini, S. Marzani, J. Rojo, and L. Rottoli, Eur. Phys. J. C 78, 321 (2018).

[35] H. Abdolmaleki et al. (xFitter Developers' Team), Eur. Phys. J. C 78, 621 (2018).

[36] H. Abramowicz et al. (H1, ZEUS Collaborations), Eur. Phys. J. C 78, 473 (2018).

[37] K. Kovařík, P. M. Nadolsky, and D. E. Soper, arXiv:1905 .06957.

[38] H.-L. Lai, M. Guzzi, J. Huston, Z. Li, P. M. Nadolsky, J. Pumplin, and C. P. Yuan, Phys. Rev. D 82, 074024 (2010).

[39] M. Aaboud et al. (ATLAS Collaboration), Eur. Phys. J. C 77, 367 (2017).

[40] J. Rojo et al., J. Phys. G 42, 103103 (2015).

[41] S. Alekhin et al., Eur. Phys. J. C 75, 304 (2015). 
[42] J. Gao, M. Guzzi, J. Huston, H.-L. Lai, Z. Li, P. Nadolsky, J. Pumplin, D. Stump, and C. P. Yuan, Phys. Rev. D 89, 033009 (2014).

[43] T. Kluge, K. Rabbertz, and M. Wobisch, arXiv:hep-ph/ 0609285.

[44] S. Catani and M. Grazzini, Phys. Rev. Lett. 98, 222002 (2007).

[45] S. Catani, L. Cieri, G. Ferrera, D. de Florian, and M. Grazzini, Phys. Rev. Lett. 103, 082001 (2009).

[46] J. M. Campbell and R. K. Ellis, Nucl. Phys. B, Proc. Suppl. 205-206, 10 (2010).

[47] R. Boughezal, J. M. Campbell, R. K. Ellis, C. Focke, W. Giele, X. Liu, F. Petriello, and C. Williams, Eur. Phys. J. C 77, 7 (2017).

[48] J. M. Campbell, R. K. Ellis, and W. T. Giele, Eur. Phys. J. C 75, 246 (2015).

[49] R. Gavin, Y. Li, F. Petriello, and S. Quackenbush, Comput. Phys. Commun. 182, 2388 (2011).

[50] R. Gavin, Y. Li, F. Petriello, and S. Quackenbush, Comput. Phys. Commun. 184, 208 (2013).

[51] Y. Li and F. Petriello, Phys. Rev. D 86, 094034 (2012).

[52] A. Gehrmann-De Ridder, T. Gehrmann, E. W. N. Glover, A. Huss, and T. A. Morgan, Phys. Rev. Lett. 117, 022001 (2016).

[53] A. Gehrmann-De Ridder, T. Gehrmann, E. W. N. Glover, A. Huss, and D. M. Walker, Phys. Rev. Lett. 120, 122001 (2018).

[54] G. A. Ladinsky and C.-P. Yuan, Phys. Rev. D 50, R4239 (1994).

[55] C. Balazs and C.-P. Yuan, Phys. Rev. D 56, 5558 (1997).

[56] S. Dulat, T.-J. Hou, J. Gao, J. Huston, J. Pumplin, C. Schmidt, D. Stump, and C. P. Yuan, Phys. Rev. D 89, 073004 (2014).

[57] A. C. Benvenuti et al. (BCDMS Collaboration), Phys. Lett. B 223, 485 (1989).

[58] A. C. Benvenuti et al. (BCDMS Collaboration), Phys. Lett. B 237, 592 (1990).

[59] M. Arneodo et al. (New Muon Collaboration), Nucl. Phys. B483, 3 (1997).

[60] J. P. Berge et al., Z. Phys. C 49, 187 (1991).

[61] U.-K. Yang et al. (CCFR/NuTeV Collaboration), Phys. Rev. Lett. 86, 2742 (2001).

[62] W. G. Seligman et al., Phys. Rev. Lett. 79, 1213 (1997).

[63] D. A. Mason, Ph. D. thesis, Oregon University, 2006.

[64] M. Goncharov et al. (NuTeV Collaboration), Phys. Rev. D 64, 112006 (2001).

[65] A. Aktas et al. (H1 Collaboration), Eur. Phys. J. C 40, 349 (2005).

[66] H. Abramowicz et al. (ZEUS, H1 Collaborations), Eur. Phys. J. C 73, 2311 (2013).

[67] G. Moreno et al., Phys. Rev. D 43, 2815 (1991).

[68] R. S. Towell et al. (NuSea Collaboration), Phys. Rev. D 64, 052002 (2001).

[69] J. C. Webb et al. (NuSea Collaboration), arXiv:hep-ex/ 0302019.

[70] F. Abe et al. (CDF Collaboration), Phys. Rev. Lett. 81, 5754 (1998).
[71] D. Acosta et al. (CDF Collaboration), Phys. Rev. D 71, 051104 (2005).

[72] V. M. Abazov et al. (D0 Collaboration), Phys. Rev. D 77, 011106 (2008).

[73] V. Abazov et al. (D0 Collaboration), Phys. Rev. D 76, 012003 (2007).

[74] T. A. Aaltonen et al. (CDF Collaboration), Phys. Lett. B 692, 232 (2010).

[75] S. Chatrchyan et al. (CMS Collaboration), Phys. Rev. D 90, 032004 (2014).

[76] S. Chatrchyan et al. (CMS Collaboration), Phys. Rev. Lett. 109, 111806 (2012).

[77] G. Aad et al. (ATLAS Collaboration), Phys. Rev. D 85, 072004 (2012).

[78] V. M. Abazov et al. (D0 Collaboration), Phys. Rev. D 91, 032007 (2015); 91, 079901(E) (2015).

[79] T. Aaltonen et al. (CDF Collaboration), Phys. Rev. D 78, 052006 (2008); 79, 119902(E) (2009).

[80] V. M. Abazov et al. (D0 Collaboration), Phys. Rev. Lett. 101, 062001 (2008).

[81] R. Aaij et al. (LHCb Collaboration), J. High Energy Phys. 08 (2015) 039.

[82] R. Aaij et al. (LHCb Collaboration), J. High Energy Phys. 05 (2015) 109.

[83] V. Khachatryan et al. (CMS Collaboration), Eur. Phys. J. C 76, 469 (2016).

[84] R. Aaij et al. (LHCb Collaboration), J. High Energy Phys. 01 (2016) 155.

[85] G. Aad et al. (ATLAS Collaboration), Eur. Phys. J. C 76, 291 (2016).

[86] S. Chatrchyan et al. (CMS Collaboration), Phys. Rev. D 90, 072006 (2014).

[87] V. Khachatryan et al. (CMS Collaboration), J. High Energy Phys. 03 (2017) 156.

[88] A. M. Sirunyan et al. (CMS Collaboration), Eur. Phys. J. C 77, 459 (2017).

[89] G. Aad et al. (ATLAS Collaboration), Eur. Phys. J. C 76, 538 (2016).

[90] P. M. Nadolsky and Z. Sullivan, eConf C010630, P510 (2001).

[91] P. M. Nadolsky, H.-L. Lai, Q.-H. Cao, J. Huston, J. Pumplin, D. Stump, W.-K. Tung, and C. P. Yuan, Phys. Rev. D 78, 013004 (2008).

[92] W. G. Seligman, Ph. D. thesis, Nevis Labs, Columbia University, 1997.

[93] U.-K. Yang et al. (CCFR/NuTeV Collaboration), J. Phys. G 22, 775 (1996).

[94] R. Aaij et al. (LHCb Collaboration), J. High Energy Phys. 06 (2012) 058.

[95] G. Aad et al. (ATLAS Collaboration), Phys. Rev. D 86, 014022 (2012).

[96] S. Chatrchyan et al. (CMS Collaboration), Phys. Rev. D 87, 112002 (2013); 87, 119902(E) (2013).

[97] V. Khachatryan et al. (CMS Collaboration), Eur. Phys. J. C 75, 288 (2015).

[98] M. Voutilainen (private communication).

[99] O. Amat et al., Proc. Sci., DIS2019 (2019) 017.

[100] S. Bailey and L. Harland-Lang, arXiv:1909.10541.

[101] B. Malaescu (private communication). 
[102] M. Kadir, A. Ablet, S. Dulat, T.-J. Hou, I. Sitiwaldi, and A. Tursun, arXiv:2003.13740.

[103] A. M. Sirunyan et al. (CMS Collaboration), J. High Energy Phys. 02 (2019) 149.

[104] G. Aad et al. (ATLAS Collaboration), J. High Energy Phys. 09 (2014) 145.

[105] V. Khachatryan et al. (CMS Collaboration), Phys. Lett. B 749, 187 (2015).

[106] V. Khachatryan et al. (CMS Collaboration), J. High Energy Phys. 02 (2017) 096.

[107] G. Aad et al. (ATLAS Collaboration), J. High Energy Phys. 08 (2016) 009.

[108] G. Aad et al. (ATLAS Collaboration), Phys. Lett. B 725, 223 (2013).

[109] G. Aad et al. (ATLAS Collaboration), J. High Energy Phys. 06 (2014) 112.

[110] G. Aad et al. (ATLAS Collaboration), J. High Energy Phys. 05 (2014) 068.

[111] S. Chatrchyan et al. (CMS Collaboration), J. High Energy Phys. 02 (2014) 013.

[112] S. Chatrchyan et al. (CMS Collaboration), J. High Energy Phys. 12 (2013) 030.

[113] V. Khachatryan et al. (CMS Collaboration), Eur. Phys. J. C 75, 147 (2015).

[114] K. J. Golec-Biernat and M. Wusthoff, Phys. Rev. D 59, 014017 (1998).

[115] F. Caola, S. Forte, and J. Rojo, Phys. Lett. B 686, 127 (2010).

[116] M. Guzzi, P. M. Nadolsky, H.-L. Lai, and C. P. Yuan, Phys. Rev. D 86, 053005 (2012).

[117] M. A. G. Aivazis, J. C. Collins, F. I. Olness, and W.-K. Tung, Phys. Rev. D 50, 3102 (1994).

[118] M. A. G. Aivazis, F. I. Olness, and W.-K. Tung, Phys. Rev. D 50, 3085 (1994).

[119] M. Kramer, F. I. Olness, and D. E. Soper, Phys. Rev. D 62 , 096007 (2000).

[120] W.-K. Tung, S. Kretzer, and C. Schmidt, J. Phys. G 28, 983 (2002).

[121] J. Gao, M. Guzzi, and P. M. Nadolsky, Eur. Phys. J. C 73, 2541 (2013).

[122] E. L. Berger, J. Gao, C. S. Li, Z. L. Liu, and H. X. Zhu, Phys. Rev. Lett. 116, 212002 (2016).

[123] Z. Nagy, Phys. Rev. D 68, 094002 (2003).

[124] V. Bertone, R. Frederix, S. Frixione, J. Rojo, and M. Sutton, J. High Energy Phys. 08 (2014) 166.

[125] M. Buza and W. van Neerven, Nucl. Phys. B500, 301 (1997).

[126] M. Czakon, D. Heymes, and A. Mitov, J. High Energy Phys. 04 (2017) 071.

[127] M. Czakon, A. Ferroglia, D. Heymes, A. Mitov, B. D. Pecjak, D. J. Scott, X. Wang, and L. L. Yang, J. High Energy Phys. 05 (2018) 149.

[128] C. Anastasiou, L. J. Dixon, K. Melnikov, and F. Petriello, Phys. Rev. D 69, 094008 (2004).

[129] C. Anastasiou, L. J. Dixon, K. Melnikov, and F. Petriello, Phys. Rev. Lett. 91, 182002 (2003).

[130] A. V. Konychev and P. M. Nadolsky, Phys. Lett. B 633, 710 (2006).

[131] J. Bellm et al., arXiv:1903.12563.
[132] S. Dittmaier, A. Huss, and C. Speckner, J. High Energy Phys. 11 (2012) 095.

[133] J. Alwall, R. Frederix, S. Frixione, V. Hirschi, F. Maltoni, O. Mattelaer, H. S. Shao, T. Stelzer, P. Torrielli, and M. Zaro, J. High Energy Phys. 07 (2014) 079.

[134] W. Hollik, B. A. Kniehl, E. S. Scherbakova, and O. L. Veretin, Nucl. Phys. B900, 576 (2015).

[135] S. Kallweit, J. M. Lindert, S. Pozzorini, M. Schönherr, and P. Maierhöfer, arXiv:1505.05704.

[136] M. Czakon, D. Heymes, A. Mitov, D. Pagani, I. Tsinikos, and M. Zaro, J. High Energy Phys. 10 (2017) 186.

[137] http://www.precision.hep.phy.cam.ac.uk/results/ttbarnnloqed-nloew/.

[138] S. Moch, J. A. M. Vermaseren, and A. Vogt, Nucl. Phys. B688, 101 (2004).

[139] A. Vogt, S. Moch, and J. A. M. Vermaseren, Nucl. Phys. B691, 129 (2004).

[140] J. Ablinger, A. Behring, J. Blümlein, A. De Freitas, A. von Manteuffel, and C. Schneider, Nucl. Phys. B890, 48 (2014).

[141] J. Ablinger, A. Behring, J. Blümlein, A. De Freitas, A. von Manteuffel, and C. Schneider, Nucl. Phys. B922, 1 (2017).

[142] D. Stump, J. Huston, J. Pumplin, W.-K. Tung, H. Lai, S. Kuhlmann, and J. Owens, J. High Energy Phys. 10 (2003) 046.

[143] R. D. Ball et al., J. High Energy Phys. 04 (2013) 125.

[144] G. D’Agostini, Nucl. Instrum. Methods Phys. Res., Sect. A 346, 306 (1994).

[145] G. D’Agostini, Bayesian Reasoning in High-Energy Physics: Principles and Applications (CERN-99-3,1999).

[146] H.-L. Lai, P. M. Nadolsky, J. Pumplin, D. Stump, W.-K. Tung, and C. P. Yuan, J. High Energy Phys. 04 (2007) 089.

[147] F. Olness, J. Pumplin, D. Stump, J. Huston, P. M. Nadolsky, H.-L. Lai, S. Kretzer, J. F. Owens, and W.-K. Tung, Eur. Phys. J. C 40, 145 (2005).

[148] R. D. Ball, V. Bertone, L. Del Debbio, S. Forte, A. Guffanti, J. I. Latorre, S. Lionetti, J. Rojo, and M. Ubiali, Phys. Lett. B 707, 66 (2012).

[149] L. A. Harland-Lang, A. D. Martin, P. Motylinski, and R. S. Thorne, Eur. Phys. J. C 75, 435 (2015).

[150] T.-J. Hou, S. Dulat, J. Gao, M. Guzzi, J. Huston, P. Nadolsky, C. Schmidt, J. Winter, K. Xie, and C. P. Yuan, J. High Energy Phys. 02 (2018) 059.

[151] T.-J. Hou et al., J. High Energy Phys. 03 (2017) 099.

[152] J. Butterworth et al., J. Phys. G 43, 023001 (2016).

[153] H.-W. Lin et al., Prog. Part. Nucl. Phys. 100, 107 (2018).

[154] M. Constantinou et al., arXiv:2006.08636.

[155] P. Amaudruz et al. (New Muon Collaboration), Phys. Rev. Lett. 66, 2712 (1991).

[156] M. Arneodo et al. (New Muon Collaboration), Phys. Rev. D 50, R1 (1994).

[157] K. Gottfried, Phys. Rev. Lett. 18, 1174 (1967).

[158] J. Liang, M. Sun, Y.-B. Yang, T. Draper, and K.-F. Liu, arXiv:1901.07526.

[159] M. Gockeler, R. Horsley, D. Pleiter, P. E. L. Rakow, and G. Schierholz (QCDSF Collaboration), Phys. Rev. D 71, 114511 (2005). 
[160] D. Dolgov et al. (LHPC, TXL Collaborations), Phys. Rev. D 66, 034506 (2002).

[161] C. Alexandrou, S. Bacchio, M. Constantinou, J. Finkenrath, K. Hadjiyiannakou, K. Jansen, G. Koutsou, H. Panagopoulos, and G. Spanoudes, Phys. Rev. D 101, 094513 (2020).

[162] Y.-B. Yang, J. Liang, Y.-J. Bi, Y. Chen, T. Draper, K.-F. Liu, and Z. Liu, Phys. Rev. Lett. 121, 212001 (2018).

[163] Y.-B. Yang, M. Gong, J. Liang, H.-W. Lin, K.-F. Liu, D. Pefkou, and P. Shanahan, Phys. Rev. D 98, 074506 (2018).

[164] M. Deka, T. Streuer, T. Doi, S. J. Dong, T. Draper, K. F. Liu, N. Mathur, and A. W. Thomas, Phys. Rev. D 79, 094502 (2009).

[165] M. Gockeler, R. Horsley, E.-M. Ilgenfritz, H. Perlt, P. E. L. Rakow, G. Schierholz, and A. Schiller, Phys. Rev. D 53, 2317 (1996).

[166] A. D. Martin, W. J. Stirling, R. S. Thorne, and G. Watt, Eur. Phys. J. C 63, 189 (2009).

[167] CTEQ-TEA, https://ct.hepforge.org/PDFs/ct18/figures/ L2Sensitivity/.

[168] A. Gehrmann-De Ridder, T. Gehrmann, E. W. N. Glover, A. Huss, and T. A. Morgan, J. High Energy Phys. 11 (2016) 094; 10 (2018) 126(E).

[169] A. Gehrmann-De Ridder, T. Gehrmann, E. W. N. Glover, A. Huss, and T. A. Morgan, Proc. Sci., LL2016 (2016) 056 [arXiv:1607.01749].

[170] A. Gehrmann-De Ridder, T. Gehrmann, N. Glover, A. Huss, and T. A. Morgan, Proc. Sci., RADCOR2015 (2016) 075 [arXiv:1601.04569].

[171] A. Gehrmann-De Ridder, T. Gehrmann, E. W. N. Glover, A. Huss, and T. A. Morgan, J. High Energy Phys. 07 (2016) 133.

[172] S. Forte and Z. Kassabov, Eur. Phys. J. C 80, 182 (2020).

[173] M. Czakon, S. Dulat, T.-J. Hou, J. Huston, A. Mitov, A. S. Papanastasiou, I. Sitiwaldi, Z. Yu, and C. P. Yuan, arXiv:1912.08801.

[174] T. Gottschalk, Phys. Rev. D 23, 56 (1981).

[175] M. Gluck, S. Kretzer, and E. Reya, Phys. Lett. B 398, 381 (1997); 405, 392(E) (1997).

[176] J. Blumlein, A. Hasselhuhn, P. Kovacikova, and S. Moch, Phys. Lett. B 700, 294 (2011).

[177] J. C. Collins, Phys. Rev. D 58, 094002 (1998).

[178] J. Gao, J. High Energy Phys. 02 (2018) 026.

[179] R. D. Ball, E. R. Nocera, and R. L. Pearson (NNPDF Collaboration), Eur. Phys. J. C 79, 282 (2019).

[180] A. Arbuzov, D. Bardin, S. Bondarenko, P. Christova, L. Kalinovskaya, U. Klein, V. Kolesnikov, L. Rumyantsev, R. Sadykov, and A. Sapronov, JETP Lett. 103, 131 (2016).
[181] G. Aad et al. (ATLAS Collaboration), J. High Energy Phys. 08 (2016) 009.

[182] C. A. Aidala et al. (SeaQuest Collaboration), Nucl. Instrum. Methods Phys. Res., Sect. A 930, 49 (2019).

[183] M. Czakon, P. Fiedler, and A. Mitov, Phys. Rev. Lett. 110, 252004 (2013).

[184] M. Czakon and A. Mitov, Comput. Phys. Commun. 185, 2930 (2014).

[185] C. Anastasiou, S. Buehler, F. Herzog, and A. Lazopoulos, J. High Energy Phys. 12 (2011) 058.

[186] J. Isaacson, B. Yan, and C.-P. Yuan, ResBos-2: Precision Resummation for the LHC Era (to be published).

[187] N. Yalkun and S. Dulat, Chin. Phys. C 43, 123101 (2019).

[188] A. Accardi et al., Eur. Phys. J. A 52, 268 (2016).

[189] CT18 PDFs, https://ct.hepforge.org.

[190] LHAPDF6, https://lhapdf.hepforge.org/.

[191] LHAPDF5, http://hep.pa.msu.edu/cteq/public/ct14/ lhapdf/v5/.

[192] J. C. Collins and J. Pumplin, arXiv:hep-ph/0105207.

[193] V. Barone, C. Pascaud, and F. Zomer, Eur. Phys. J. C 12, 243 (2000).

[194] S. Alekhin, J. Blümlein, and S. Moch, Phys. Lett. B 777, 134 (2018).

[195] F. James and M. Winkler, http://seal.web.cern.ch/seal/ documents/minuit/mnusersguide.pdf (2004).

[196] T. Hahn, Comput. Phys. Commun. 168, 78 (2005).

[197] L. A. Harland-Lang, A. D. Martin, and R. S. Thorne, Eur. Phys. J. C 78, 248 (2018).

[198] V. Khachatryan et al. (CMS Collaboration), J. Instrum. 12, P02014 (2017).

[199] H. Paukkunen and P. Zurita, J. High Energy Phys. 12 (2014) 100.

[200] S. Camarda et al. (HERAFitter developers' Team), Eur. Phys. J. C 75, 458 (2015).

[201] Fitter developers, https://www.xfitter.org/xFitter/xFitter/ DownloadPage action=AttachFile $\& d o=$ view $\&$ target $=$ manual.pdf (2017).

[202] S. Catani and M. H. Seymour, Phys. Lett. B 378, 287 (1996).

[203] S. Catani and M. H. Seymour, Nucl. Phys. B485, 291 (1997); B510, 739 (1998).

[204] T. Binoth and G. Heinrich, Nucl. Phys. B585, 741 (2000).

[205] C. Anastasiou, K. Melnikov, and F. Petriello, Phys. Rev. D 69, 076010 (2004).

[206] C. Anastasiou, K. Melnikov, and F. Petriello, Nucl. Phys. B724, 197 (2005). 